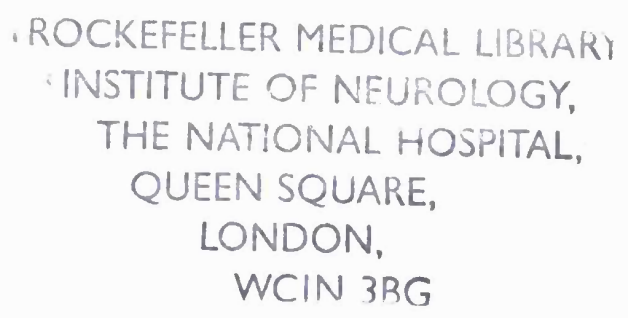

Long term clinical and neurophysiological follow-up of patients with peripherai neuropathy associated with benign monoclonal gammopathy

\author{
Sawanthana Ponsford \\ MB, BSc, FRCP
}

\title{
MD THESIS
}

2000

Submitted to the University of London 
ProQuest Number: U145714

All rights reserved

INFORMATION TO ALL USERS

The quality of this reproduction is dependent upon the quality of the copy submitted.

In the unlikely event that the author did not send a complete manuscript and there are missing pages, these will be noted. Also, if material had to be removed, a note will indicate the deletion.

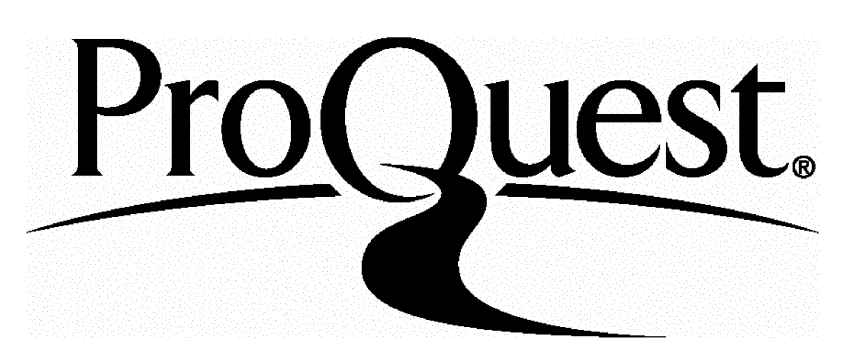

ProQuest U145714

Published by ProQuest LLC(2015). Copyright of the Dissertation is held by the Author.

All rights reserved.

This work is protected against unauthorized copying under Title 17, United States Code. Microform Edition (c) ProQuest LLC.

\author{
ProQuest LLC \\ 789 East Eisenhower Parkway \\ P.O. Box 1346 \\ Ann Arbor, MI 48106-1346
}


In search of knowledge

In the hope of obtaining wisdom 


\begin{abstract}
The primary aim of this study was to determine the long term clinical outcome including the incidence of haematological malignancy in a group of patients initially diagnosed as having neuropathy associated with a benign monoclonal gammopathy. The evolution of the neurophysiological findings over time was also examined. In addition an attempt was made to delineate individual nosological subgroups based on the clinical course and the immunological and neurophysiological findings.
\end{abstract}

Fifty patients with peripheral neuropathy associated with benign monoclonal gammopathy were studied. The duration of the follow-up period was $5-35$ years. $74.5 \%$ had an $\operatorname{lgM}$ paraprotein ( $\kappa$ light chain in $64 \%$ ). The remainder either had an $\lg G$ or ( 1 case only) an $\lg A$ paraprotein.

Fifteen patients had died. Only $6 \%$ of patients developed haematological malignancy in comparison with the reported $17-25 \%$ in patients ascertained by the presence of a paraprotein in the absence of neuropathy. None had multiple myeloma. All had been on immunosuppressive therapy for at least 2 years before the diagnosis of the malignancy. The highest incidence of malignancy of all types (44\%) was for the gastrointestinal tract.

Of 24 survivors with benign IgM paraproteinaemic neuropathy, $29 \%$ had only myelin associated glycoprotein antibodies with typical male predominance, late onset, and a progressive distal sensorimotor polyneuropathy. Evoked distal muscle amplitudes were significantly smaller and the relative prolongation of distal motor latency observable in the early cases was no longer apparent. This suggested progressive axonal degeneration indicating a less favourable prognosis.

Two patients (8.3\%) with IgM antidisialosyl antibodies and cold agglutinin activity had a large fibre neuropathy with intermittent oculofacial involvement responding to intravenous immunoglobulin 
treatment. The above two categories thus formed separate clinical and immunological entities. Findings in the remaining patients were varied. Some were not dissimilar to patients with chronic inflammatory demyelinating polyneuropathy. Recognition of $\operatorname{lgM}$ subgroups is important both for prognosis and possible response to treatment. 


\section{Acknowledgements}

The research described in this Thesis was conducted in the Out Patient Department, The National Hospital for Neurology and Neurosurgery, the Department of Clinical Neurophysiology, Royal Free Hospital, London, the patients' homes and nursing homes, under the supervision of Professor P K Thomas.

I expressed my most sincere gratitude to all the patients and their relatives, matrons and staff of the nursing homes and all the staff in the Medical Records Department in various hospitals especially Mr G Collins of the National Hospital for Neurology and Neurosurgery and to Mrs Jean Veitch. The cooperation of the General Practitioners, Office for National Statistics and the help and advice from $\mathrm{Dr} \mathrm{H} J$ Willison, $\mathrm{Dr} J$ Scott, Dr R H M King, Mr T Adams, Dr L Ginsberg, Dr H Manji, Dr J G Llewelyn, Mrs G Geeta and Sister Sylvia Curry are very much appreciated. The Thesis would not have been completed without the helpful discussions and guidance of Professor $P K$ Thomas whose example continues to give inspiration. Last but not least, I would like to thank both my son for being good and understanding, and also those who hindered, thus inadvertently providing more strength and courage throughout a difficult time.

I am most grateful to Mr Pete James of The Department of Medical Illustration, Coventry and Warwickshire Hospital, Walsgrave Hospital NHS Trust and Mr J Muddle for their help with the illustrations.

The project was approved by the local ethical committee at the National Hospital for Neurology and Neurosurgery and all patients gave informed consent. 


\section{Declaration of author's contribution}

The collection of the clinical and neurophysiological data was performed by the author. The majority of the final assessments for each patient were performed at the patients' homes or nursing homes, which were widely scattered in the UK from Middlesbrough to Penzance.

I am indebted to $\mathrm{Dr} \mathrm{H} J$ Willison and Mrs Jean Veitch for performing the immunological studies.

The clinical notes and questionnaires were reviewed and obtained with consent from the physicians and general practitioners, many with the help of the patients and their close relatives. In a few cases, the followup would have been impossible without the help of the Medical Records Department, the National Hospital for Neurology and Neurosurgery and the Royal Free Hospital, and the Office for National Statistics. Dr Richard Morris, Department of Primary Care and Population Sciences, the Royal Free and University College Medical School kindly gave advice on statistical analysis. The pathological material was reviewed by the author and the illustrations were generously provided by $\mathrm{Dr} J$ Jacobs, Institute of Neurology, Queen Square and Dr R H M King, The Royal Free and University College Medical School. 


\section{PUBLICATIONS:}

PONSFORD S, WILLISON H, VEITCH J, MORRIS R, THOMAS PK (2000) Long-term clinical and neurophysiological follow-up of patients with peripheral neuropathy associated with benign monoclonal gammopathy. Muscle Nerve, 23, 164-174.

PONSFORD S, WILLISON H, THOMAS PK (2000) CANOMAD. J Neurol (in press). 


\section{CONTENTS}

Abstract 3

Acknowledgements $\quad 5$

$\begin{array}{ll}\text { Declaration of the author's contribution } & 6\end{array}$

$\begin{array}{ll}\text { Publications } & 7\end{array}$

Contents

\section{CHAPTER 1: INTRODUCTION}

$\begin{array}{ll}1.1 \text { Background } & 13\end{array}$

1.2 Neuropathy associated with benign monoclonal gammopathy 15

1.3 Benign IgM paraproteinaemic neuropathy 16

Benign IgM paraproteinaemic neuropathy with antiMAG activity 17

$\begin{array}{ll}\text { Clinical features } & 18\end{array}$

$\begin{array}{ll}\text { Neurophysiological findings } & 19\end{array}$

Predominantly motor neuropathy with IgM activity against GM1 gangliosides 21

Clinical features $\quad 22$

Neurophysiological findings $\quad 24$

Chronic ataxic neuropathy with benign IgM paraprotein activity against disialosyl gangliosides 26

Clinical features $\quad 26$

Neurophysiological findings $\quad 28$

Other laboratory results $\quad 29$

Chronic sensory neuropathy with benign IgM activity against sulphatides 29

Clinical features $\quad 30$

Neurophysiological findings $\quad 31$

Neuropathy with benign IgM paraproteinaemia reacting with chondroitin sulphate 31

Other IgM antibody activities $\quad 32$

1.4 Benign $\lg G$ or $\lg A$ or $\lg D$ paraproteinaemic neuropathy 32 
1.5 Neuropathology of benign paraproteinaemic neuropathy

1.6 Long term outcome for the incidence of haematological malignancy

1.7 Aims of present study 38

Table $1.1 \quad 40$

Table 1.2

Fig. $1.1 \quad 42$

Fig. 1.2

\section{CHAPTER 2: PATIENTS AND METHODS}

2.1 Patients

Recruitment $\quad 46$

Inclusion criteria $\quad 46$

2.2 Methods

Clinical assessment $\quad 47$

Neurophysiology $\quad 47$

Criteria for the diagnosis of demyelinating neuropathy 48

Criteria for the diagnosis of axonal degeneration 49

Immunological investigations

Assays for antimyelin associated glycoprotein antibody $\quad 49$

Ganglioside antibody assays $\quad 49$

LM1 50

Immunoabsorption and purification for cold agglutinating antibody 51

Histopathological investigations $\quad 52$

Statistical methods $\quad 52$

Symptoms score $\quad 54$

Sign score $\quad 55$

Scoring for clinical disability $\quad 56$

Proforma I $\quad 57$

Proforma II 61

Questionnaire to physician or general practitioner 63

Questionnaire to patient $\quad 66$ 


\section{CHAPTER 3: RESULTS}

\subsection{Patients}

Demographic data $\quad 71$

$\begin{array}{ll}\text { Deceased patients } & 71\end{array}$

Surviving patients

Immunological subclassification $\quad 72$

Clinical features (including the deceased patients) 73

Results of summed scores $\quad 75$

$\begin{array}{lll}3.2 & \text { Neurophysiological findings } & 75\end{array}$

$\begin{array}{ll}\text { Sensory study } & 76\end{array}$

Motor study

Motor conduction velocity $\quad 76$

Distal motor latency $\quad 77$

Distal compound muscle action potentials

Residual latency

Terminal latency index

Other neurophysiological findings $\quad 78$

$\begin{array}{lll}3.3 & \text { Other laboratory findings } & 78\end{array}$

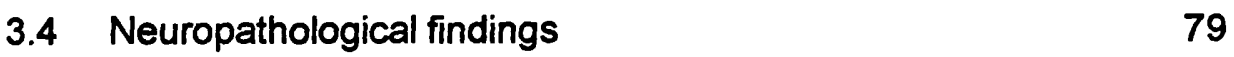

3.5 Illustrative cases 80

Table $3.1 \quad 89$

Table $3.2 \quad 91$

Table $3.3 \quad 92$

Table $3.4 \quad 93$

Table $3.5 \quad 93$

Table $3.6 \quad 94$

Table $3.7 \quad 95$

Table $3.8 \quad 96$

Table $3.9 \quad 98$

Table $3.10 \quad 99$

Table $3.11 \quad 99$

$\begin{array}{ll}\text { Fig. } 3.1 & 100\end{array}$

Fig $3.2 \quad 106$ 
Fig 3.3

Fig 3.4

Fig 3.5

Fig 3.6

CHAPTER 4: DISCUSSION

4.1 Summary of the findings

Demographic data 133

Incidence of malignancy

133

Clinical features

134

Summed scores

135

Neurophysiological findings

135

Other laboratory findings

135

Neuropathological findings

136

4.2 Discussion

136

CHAPTER 5: CONCLUSIONS

REFERENCES 


\section{CHAPTER 1}

\section{INTRODUCTION}

\subsection{Background}

1.2 Neuropathy associated with benign monoclonal gammopathy

1.3 Benign IgM paraproteinaemic neuropathy

- Benign IgM paraproteinaemic neuropathy with antiMAG activity

Clinical features

Neurophysiological findings

- Predominantly motor neuropathy with IgM activity against GM1 gangliosides

Clinical features

Neurophysiological findings

- Chronic ataxic neuropathy with benign IgM paraprotein activity against disialosyl gangliosides

Clinical features

Neurophysiological findings

Other laboratory results

- Chronic sensory neuropathy with benign IgM activity against sulphatides

Clinical features

Neurophysiological findings

- Neuropathy with benign IgM paraproteinaemia reacting with chondroitin sulphate

- Other IgM antibody activities

1.4 Benign IgG and IgA paraproteinaemic neuropathy

1.5 Neuropathology of benign paraproteinaemic neuropathy

1.6 Long term outcome on the incidence of haematological malignancy

1.7 Aims of present study 


\section{INTRODUCTION}

\subsection{Background}

Paraproteins are monoclonal immunoglobulins $(\mathrm{lg})$ produced in excess by abnormal proliferation of a single clone of plasma cells or their monoclonal B cell precursors. Each monoclonal protein can consist of the intact immunoglobulin structure (2 heavy polypeptide chains of the same class and subclass and 2 light polypeptide chains of the same type) from any of the 5 immunoglobulin isotypes: $\lg M, \lg G, \lg A, \lg E, \lg D$ although most commonly from the first three (Fig.1.1, Table 1.1). The paraprotein can also occur as free kappa $(\kappa)$ or lambda $(\lambda)$ light chains. These can at times be deposited in peripheral nerve as amyloid fibrils when the material adopts a beta-pleated molecular configuration. Occasionally more than one paraprotein may be present and this phenomenon is referred to as biclonal (llyas et al, 1988; Kyle, 1995) or triclonal gammopathy (Kyle, 1995). This is different from polyclonal gammopathy in which there is a diffuse increase in immunoglobulins.

The proliferation of the $\mathrm{B}$ cell or its precursors may be benign or malignant, the latter leading to various haematological disorders: multiple or osteosclerotic myeloma, B cell lymphoma, benign plasma cell dyscrasia, chronic lymphocytic leukaemia, Waldenström macroglobulinaemia. The relative incidence of the benign to malignant gammopathies is approximately 200 to 1 (Radl, 1985). Nonmalignant monocional gammopathies have also been referred to as "benign monoclonal gammopathy" or "monoclonal gammopathy of undetermined significance" (MGUS) (Kyle, 1978; Kyle, Dyck, 1993) as the distinction between benign and malignant gammopathies may be difficult and the progression may be gradual. MGUS is characterised by a serum paraprotein less than $30 \mathrm{~g} / \mathrm{L}$, plasma cells of less than $10 \%$ in the bone marrow, no Bence-Jones proteinuria, no lytic lesions and no evidence of immunosuppression. 
Monocional gammopathy is found, on average, in $1 \%$ of the normal adult population (Kyle, 1995) and is usually lgG, in about $60 \%$, IgM in 10-27\% and IgA the remainder (Ameis et al., 1976; Fine et al., 1978; Kahn et al., 1981; Kyle et al., 1972; Radl, 1985). There is an increasing prevalence with age: $0.1 \%$ in the third decade to $3 \%$ in the eighth decade and 19\% after the age of 95 (Axelsson et al., 1966; Fine et al., 1978; Kyle et al., 1972; Radl et al., 1975; Radl, 1985; Saleun et al., 1982). The incidence is higher in African-Americans than in Caucasians and is less in Japanese (Kyle, 1995). The detection of the presence or absence of a paraprotein clearly depends on the sensitivity of the detection technique and at least serum protein electrophoresis, immunoelectrophoresis or immunofixation of serum and urine should be performed (Kelly, Jr., 1985) because the concentration of the paraprotein may be very low especially when associated with polyneuropathy.

In a 24-38 y follow-up of 241 patients found to have benign monoclonal gammopathy before 1971 (Kyle, 1995), 26\% developed lymphoproliferative disorders with the calculated risk of serious disease being $16 \%, 33 \%$ and $40 \%$ at 10,20 and 40 y respectively. The calculated risk was higher in IgM than in IgA or IgG gammopathies $(52 \%, 37 \%, 29 \%$ respectively) at $20 y$ with a median interval from recognition of the paraprotein to the development of malignancy of $8.5 \mathrm{y}$ (4-20y). The risk clearly increases with duration of the disease. The malignant IgG and IgA monoclonal gammopathies are usually associated with multiple myeloma and rarely with lymphoma (Kyle et al., 1969; Linos et al., 1981). Malignant IgM monoclonal paraproteins are usually associated with Waldenström macroglobulinaemia (Kyle, 1995) and occasionally with B cell lymphoma or chronic lymphocytic leukaemia (Azar et al., 1957; Qian et al., 1984). 


\subsection{Neuropathy associated with benign monoclonal gammopathy}

Forssman et al (1973) reported the first case of benign IgM monoclonal gammopathy and peripheral neuropathy. Nerve biopsy revealed IgM deposits as well as IgM producing lymphocytes. The patient improved with chlorambucil therapy. As paraproteins also occur in the normal population, a chance association was a possibility. It was not until Kahn et al in 1980, using agarose electrophoresis, found a disproportionately high incidence $(28 \%)$ of unexplained peripheral neuropathy in association with benign monoclonal gammopathy amongst 14,000 sera that a clear association emerged. A statistically significant increase in the prevalence of peripheral neuropathy in patients with benign monoclonal gammopathy in comparison with the normal population was subsequently noted at the Mayo Clinic, Minnesota (Kelly, Jr. et al., 1981) and in Sweden (Ösby et al., 1982). The overall incidence of peripheral neuropathy associated with benign monoclonal gammopathy is difficult to estimate but is probably between $5-25 \%$ (Thomas, Willison, 1994). On the other hand, $10 \%$ of patients with idiopathic polyneuropathy have an associated monoclonal gammopathy (Kelly, Jr. et al., 1981; Latov, 1995). The incidence of neuropathy varied depending on the protein isotypes occurring in $31 \%$ of patients with IgM monoclonal gammopathy and 6 and $14 \%$ of patients with $\lg G$ and $\lg A$ paraproteinaemia respectively (Latov, 2000).

A causal link between the IgM paraproteins and peripheral neuropathy was suggested by the initial observation of the $\operatorname{lgM}$ paraprotein binding to myelin (Kahn et al., 1981; Latov et al., 1980; Leibowitz et al., 1983; Meier et al., 1984a; Smith et al., 1983) with colocalisation of $\mathrm{C} 1 \mathrm{q}, \mathrm{C} 3 \mathrm{~d}, \mathrm{C} 5$ and the terminal complement components (Ellie et al., 1996; Monaco et al., 1990) and the subsequent demonstration of specific IgM antibodies activities against carbohydrate antigens on cell surface glycoconjugates: myelin associated glycoprotein (MAG) (Latov, 1990a; Latov, 1990b; Nobile-Orazio et al., 
1994) and gangliosides (Pestronk et al., 1988). Although passive transfer of antiMAG antibodies failed to produce neuropathy in experimental animals (Steck et al., 1985) and attempts to induce in vivo animal models have met with limited success (llyas et al., 1990; O'Shannessy et al., 1985), intraneural injection of the antiMAG paraprotein and complement in cat sciatic nerve has been shown to produce demyelination (Hays et al., 1987; Trojaborg et al., 1995; Willison et al., 1988). Successful production of the neuropathological changes observed in the peripheral nerves in man was also obtained by the injection of antiMAG IgM into the peritoneum of $2 \mathrm{~d}$ chicks (Tatum, 1993).

The identified potential target antigens for patients with IgG or IgA paraproteins have been investigated far less extensively than for IgM paraproteinaemia. The monoclonal IgG paraproteins in 4 patients with axonal sensorimotor neuropathy were reported to react with the 68 kDa axonal neurofilament protein (Fazio et al., 1992; Nemni et al., 1990). The IgA paraprotein in a patient with a motor neuron diseaselike illness showed reactivity with a neurofilament antigen (Hays et al., 1990).

\subsection{Benign IgM paraproteinaemic neuropathy}

Patients with benign IgM paraproteinaemic neuropathy can be distinguished from those with IgG/A. Gosselin et al (1991) and Suarez et al (1993) found those with IgM paraproteins to have higher frequency of sensory loss and more consistent neurophysiological evidence of demyelination. Slower motor conduction velocity in the peroneal nerve and the more frequently absent ulnar sensory action potentials (SAP) were also observed by Simovic et al (1998) in their 5 y follow-up of 19 patients with benign IgM paraproteinaemic neuropathy in comparison with 15 patients with IgG paraproteinaemia. Notermans et al (1994) noted that the polyneuropathy progressed more rapidly leading to significant motor and sensory deficits in the former. Yeung et al (1991) 
found that a major difference between the $3 \mathrm{lg}$ classes was the detection of immunoglobulin on myelin in nerve biopsies from patients with IgM paraproteinaemia, more commonly in those with a $\kappa$ light chain. The same was true for abnormal widening of the myelin periodicity. In addition, the IgM paraproteins have more consistently identifiable antibody activity.

Several subgroups of benign IgM paraproteinaemic neuropathy with particular clinical and serological associations have been recognised and these will now be discussed.

\section{Benign IgM paraproteinaemic neuropathy with antiMAG activity}

Myelin associated glycoprotein is a well known 100-kDa glycoprotein selectively localised in the periaxonal membranes of Schwann cells, Schmidt-Lanterman incisures, terminal loops, at nodes of Ranvier, and inner and outer mesaxons and is absent from compact myelin. MAG has 5 extracellular immunoglobulin-like domains, a single transmembrane domain and a cytoplasmic domain (Fig. 1.2) (Quarles, Weiss, 1999). Its oligosaccharide determinant reacts with the HNK-1 adhesion molecule that is shared by the nervous and immune systems. Additional shared antigens include the major glycoprotein in compact myelin, the myelin protein zero (Po, MPZ), peripheral myelin protein 22 (PMP 22), the glycolipid sulphoglucuronyl paragloboside (SGPG) and sulphoglucuronyl lactosaminyl paragloboside (SGLPG)(Latov, 1990a).

In $50-60 \%$ of patients with benign IgM paraproteinaemic neuropathy, the IgM paraprotein reacts with MAG (Latov, 1990a; Quarles et al., 1986). Braun et al (1982) first demonstrated IgM paraproteins reacting with MAG. Several confirmations followed: (Meier et al., 1984a; Meier et al., 1984b; Mendell et al., 1985; Steck et al., 1983; Stefansson et al., 1983). Selective loss of MAG in myelinated fibres was found to be inversely related to the serum antiMAG titres (Gabriel et al., 1996). The shared reactive epitope on peripheral nerve glycolipids was observed by llyas et al (1984), Chou et al (1986) and 
Ariga et al (1987); neural cell adhesion molecule by Noronha et al (1986); J1 glycoprotein by Poltorak et al (1986); and the Po glycoprotein by Bollensen et al (1988). The antigenic site involves the 3-sulphateglucuronic acid residue (llyas et al., 1986a). Some antiMAG also cross reacts with sulphatide (llyas et al., 1992; Pestronk et al., 1991) and may be present in the absence of any recognisable paraproteinaemia.

\section{Clinical features}

Patients who have IgM benign paraproteinaemic neuropathy with antibody activities against MAG are usually male with onset of symptoms in their late fifth or sixth decade. They typically present with a distal and symmetrical sensory or sensorimotor polyneuropathy (Ellie et al., 1996; Hafler et al., 1986; Latov, 1995; Maisonobe et al., 1996; Notermans et al., 1994; Smith, 1994; van den Berg et al., 1992; van den Berg et al., 1996). A few patients may have prominent motor involvement (Antoine et al., 1993; Ellie et al., 1996). Tremor may occur early in the disease. Upper limbs tremor is found up to $90 \%$ of patients with benign IgM paraproteinaemic neuropathy (Dalakas et al., 1984; Léger, Vaunaize, 1990; Léger et al., 1992a; Smith et al., 1983; Smith, 1994; Yeung et al., 1991). It is usually an action or postural tremor predominantly affecting distal rather than proximal muscles and is often irregular and variable in amplitude (Smith et al., 1983). Cranial nerves, central nervous system and autonomic function are usually spared. Progression is slow, less so during the initial 2-5 y. All patients eventually developed motor and sensory signs and ataxia resulting in significant disability. Smith (1994) observed an improvement or resolution of unpleasant paraesthesiae in more than half of his patients. Nobile-Orazio et al (1994) did not find any clinical correlation between the antiMAG titres and the severity of the neuropathy. 
Neurophysiological findings

Despite the heterogeneous neurophysiological findings in patients with benign IgM paraproteinaemic neuropathy, this particular subgroup of patients with IgM antiMAG activity shows relatively uniform electrodiagnostic changes. The first findings were reported by Kelly (Kelly, Jr., 1990) comparing 7 patients with IgM paraproteins and antiMAG activity with 7 patients with IgM paraproteins but no antiMAG activity. Not all the clinical details (including the duration from the onset of symptoms) of all the patients were given. At least 3 of the 4 following criteria were used for the diagnosis of demyelination:

(1) Conduction velocity slowing in 2 or more motor nerves to less than $65 \%$ of the normal mean value for that nerve (or $70 \%$ of the lower limits of normal.

(2) Distal latency prolongation in 2 or more motor nerves exceeding $130 \%$ of the upper normal limits.

(3) Evidence of temporal dispersion ( $>15 \%$ increase in duration) or conduction block in the absence of temporal dispersion ( $>30 \%$ decrease in compound muscle potentials) with proximal stimulation in 2 or more motor nerves.

(4) F response minimal latency exceeding $130 \%$ of the upper limits of the normal in 2 or more nerves.

Using only the median nerve for analysis, 5 of the 6 patients with antiMAG activity met the above criteria whilst only 1 patient without antiMAG activity did. Mean median motor conduction velocity (MCV) and distal motor latency (DML) were 35 and $42 \mathrm{~m} / \mathrm{s}, 7.9$ and $6.6 \mathrm{~ms}$ for the former and latter groups respectively. The differences were statistically significant $(P<0.05)$. There was no statistically significant difference in the distal compound muscle action potentials between the 2 groups ( 5.4 and $5.3 \mathrm{mV}$ ). Conduction block was found in 2 cases in the antiMAG positive and 1 in the antiMAG negative group. Sensory action potentials (SAP) were said to be low or absent and motor responses were better preserved in the arms. 
Kaku and Sumner (1992) and Kaku et al (1994) found a marked prolongation of distal motor latencies disproportionate to the proximal conduction velocity in chronic neuropathy associated with monoclonal IgM antibodies to MAG and SGPG. Seventy six percent (16 out of 21 nerves from 4 patients) of such patients also had a terminal latency index (TLI) $\leq 0.25$ in comparison with $6 \%$ (11 out of 195 nerves) of patients with type $1 \mathrm{~A}$ Charcot-Marie-Tooth disease, $6 \%$ (3 out of 49 nerves) of patients with chronic inflammatory demyelinating polyneuropathy (CIDP) and none of the normal controls $(P=0.001)$. TLI was calculated from terminal distance divided by conduction velocity times terminal latency (see Chapter 2). All 4 patients with benign paraproteinaemic neuropathy with IgM paraproteins reactive against MAG and SGPG had had symptoms for between 15 mo to 5 y (mean $11.2 \mathrm{mo}$ ).

Trojaborg et al (1995) observed a similar change of shorter TLI, longer residual latency (RL) and slower MCV in patients with high antiMAG titres than those with lower titres or other neuropathies. Only sural nerve biopsies in patients with high antiMAG titres showed IgM deposits and complement on the myelin sheaths, pronounced demyelination and widely spaced myelin lamellae.

Ellie et al in 1996 studied 30 patients with peripheral neuropathy and IgM paraproteinaemia with antiMAG activity and 3 patients with biclonal antiMAG gammopathy with a mean duration of symptoms of 72 mo (46-81 mo). Patients were divided into 3 groups according to their symptoms and signs: pure sensory neuropathy, sensorimotor neuropathy with mild weakness and sensorimotor neuropathy with severe weakness. There were significant differences statistically in the median and ulnar MCV, DML and RL between groups I and II and groups I and III, but not between groups II and III (median MCV:37, 24 and $28 \mathrm{~m} / \mathrm{s}$ ). The duration of symptoms was noted to be significantly longer between groups I and II or III but not between II and III: mean 48, 88,72 mo in group I, II and III respectively. Therefore the difference in 
the neurophysiological parameters may simply have reflected the progression of the disease process. Only $42 \%$ of the nerves had a TLI value of $\leq 0.25$. Conduction block was seen in 2 cases. The slowing was comparable between nerves in the upper and lower limbs and between sides. Compound muscle action potentials were not mentioned but there were changes of mild chronic denervation on electromyographic examination of distal muscles.

The neurophysiological evidence thus far indicates that the peripheral neuropathy with benign IgM antiMAG activity is primarily demyelinating with predominant involvement in the very distal segments. Unlike CIDP, conduction block was rare and the degree of slowing in motor nerves was fairly uniform in the more proximal segments. The mild distal neurogenic change in the presence of a marked slowing of the MCVs suggests a secondary axonal degeneration. The reduction in the percentage of low $\mathrm{TLI}$ of $\leq 0.02$ in the study by Ellie et al, when the duration of the disease was longer than for those in Trojaborg's study, indicates fewer cases with prominent terminal segment involvement, possibly as a result of progression of the underlying pathology proximally.

\section{Predominantly motor neuropathy with IgM activity against GM1}

\section{gangliosides}

Gangliosides are complex glycosphingolipids that contain at least one sialic acid residue, $\mathrm{N}$-acetylneuraminic acid, in man. Glycosphingolipids are composed of the long chain aliphatic amine sphingosine (acylated ceramide) attached to one or more sugars (hexoses). Gangliosides are present in brain, less so in peripheral nerve $(0.3-0.7 \%$ of total myelin lipids) and other tissues. They are particularly concentrated in neural plasma membranes and are highly enriched in the myelin sheaths of motor nerves (Ogawa-Goto et al., 1990; Ogawa-Goto et al., 1992). Gangliosides are amphiphatic molecules that occur primarily in cell 
surface membranes with their oligosaccharides exposed to the extracellular environment (Quarles, Weiss, 1999) and are thus well situated for reaction with any antibodies. The gangliosides show not only wide variation in their structure, examples of which are shown in Table 1.2 but they also vary widely across different species at different development stages with considerable functional diversity (Tettamanti, Riboni, 1993). The Gal( $\beta 1-3)$ GalNAc epitope found on GM1 is also present on asialo-GM1 and GD1b and the complete GM1 carbohydrate sequence is present on Campylobacter jejuni lipopolysaccharide. These factors are probably responsible for the diversity of the clinical expression of the antigen-antibody reaction.

Monoclonal IgM with antiGM1 activity was first reported in a patient with monoclonal IgM gammopathy and lower motor neuron disease syndrome by Freddo et al (1986) and in patients with multifocal motor neuropathy (Pestronk et al., 1988; Pestronk et al., 1990). The antiGM1 antibodies were found to differ in their fine specificities (Baba et al., 1989). Monoclonal gammopathies are less common than polyclonal elevations of GM1 antibodies. IgM paraproteinaemia is, in fact, rare in patients with GM1 antibodies (Kinsella et al., 1994; Miescher, Steck, 1996).

\section{Clinical features}

Patients with high titres of IgM antiGM1 activity have been suggested to constitute distinct clinical syndromes:

- Multifocal motor neuropathy with persistent conduction block. Lewis et al (1982) reported 5 patients with sensory and motor symptoms consistent with a demyelinating mononeuropathy multiplex. Deficits were prominent in the upper limbs and predominantly involved motor function. Although some muscles with severe weakness had normal bulk, others showed severe atrophy with fasciculations and myokymia 
simulating motor neuron disease. There was persistent and severe motor conduction block confined to short segments of the affected nerves with relatively preserved motor conduction in the intervening segments. Sensory conduction studies were mildly abnormal. Nerve biopsy was indistinguishable from CIDP. Cerebrospinal fluid (CSF) protein was mildly elevated and 2 patients improved with prednisolone. The neuropathy has become known as the Lewis-Sumner syndrome and the cases considered to be the first examples of multifocal motor neuropathy (MMN). Most of the subsequent reports described patients with pure multifocal motor neuropathies, simulating motor neuron disease (but without upper motor neuron signs), with conduction block (Auer et al., 1989; Chad et al., 1986; Feldman et al., 1991; Kaji et al., 1992; Krarup et al., 1990; Pestronk et al., 1988; Pestronk et al., 1990; Roth et al., 1986). Opinions vary regarding the relationship between the Lewis-Sumner syndrome and MMN. A recent report by Saperstein et al (1999) compared and contrasted 11 cases of Lewis-Sumner syndrome with their earlier 16 cases of MMN (Katz et al., 1997) and concluded that the 2 syndromes are distinct. This was based on the difference in the degree of clinical, neurophysiological and morphological findings with respect to sensory nerve involvement (minimal or absent in MMN), a high frequency of elevated CSF protein levels in the Lewis-Sumner syndrome, and frequently high titres of antiGM1 and lack of response to corticosteroid treatment in MMN. Doubt remains whether the 2 are separate disorders as sensory symptoms may develop long after pure motor involvement and sensory involvement is confirmed by quantitative sensory and nerve biopsy in MMN. The small number of cases ( 3 out of 6 responded to steroid therapy) 
also made the conclusion on corticosteroid treatment invalid (Parry, 1999). Certainly both are immune mediated neuropathies and share many common features with CIDP. The incidence of high IgM antiGM1 titres in MMN is approximately 44-68\% (Katz et al., 1997; Pestronk et al., 1990)

- Other lower motor neuron syndromes. Pestronk et al (1990) in their study of 74 patients with lower motor neuron syndromes suggested 2 further groups of patients. A lower motor neuron syndrome with predominantly distal weakness, no conduction block and a high incidence of antiGM1 antibodies, and a lower motor neuron syndrome with early proximal weakness and serum antibodies to asialo-GM1 that do not cross react with GM1 ganglioside. The latter was commoner in males. Seventy two percent of patients had onset later than $45 \mathrm{y}$ that was in contrast to the $68 \%$ of patients with onset before the age of 45 in MMN. The weakness often remained confined mainly to 1 or 2 limbs for 3-5 y. Conduction block was unusual and the antibody activity was against asialo-GM1 and the Gal( $(\beta 1-3)$ GalNAc disaccharide with a frequency of $50 \%$. The possibility remains that the former is $M M N$. Failure in detection of proximal conduction block may simply be due to limitations in the conventional nerve conduction techniques.

AntiGM1 antibodies are also common in amyotrophic lateral sclerosis, especially in patients with prominent lower motor neuron changes, but in lower titres (Pestronk et al., 1989).

Neurophysiological findings

Multifocal partial motor conduction block (PMCB) is considered an essential feature of the diagnosis of MMN. The diagnosis obviously 
depends on the neurophysiological criteria for PMCB. There is no agreement on the definition of PMCB. Lange et al (1992) used 2 of the following: focal reduction in amplitude or area of the compound action muscle potentials (CMAP) by more than $50 \%$ in a segment $<25 \mathrm{~cm}$; focal slowing of motor conduction velocity with normal sensory nerve conduction. Chaudhry et al (1994) defined PMCB as a $>50 \%$ reduction in both the CMAP amplitude and the negative-peak area on proximal stimulation as compared with the response obtained on distal stimulation, accompanied by no more than a $15 \%$ change in the negative-peak duration. A more stringent criterion was used by Katz et al (1997) using a reduction in amplitude and area of the CMAP of $>50 \%$ across a standard segment and a less than $30 \%$ increase in CMAP duration. This is to avoid the false positive changes due to phase cancellation from the significant loss of motor axons (Cornblath et al., 1990; Rhee et al., 1990). With a more strict criterion, only $31 \%$ of 16 patients by Katz et al (1997) had conduction block. The desire to increase specificity obviously came at the expense of the reduction of diagnostic sensitivity in nerve conduction studies.

Abnormal temporal dispersion was defined as a $>15 \%$ increase in the negative-peak duration of the proximal CMAP as compared with the distal CMAP (Chaudhry et al., 1994) or a duration increase by $>30 \%$ without a $50 \%$ in amplitude or area (Katz et al., 1997). Other changes indicative of demyelination include: motor conduction velocities $<70 \%$ of the lower limit of normal with a distal CMAP amplitude $<80 \%$ of the lower limit of normal (LLN) or MCV $<80 \%$ of the LLN when the amplitude was $>80 \%$ of the LLN. Minimum F wave and distal motor latencies were considered to be in the demyelinating range if they are $>120-125 \%$ of the upper limit of normal. Using the above criteria, several reports also showed the presence of widespread demyelinating features in the other nerves together with evidence of axonal degeneration (Katz et al., 1997; Pestronk et al., 1988; Roberts et al., 1995). Sensory action potentials are usually 
normal despite mildly but definitely abnormal sensory nerve biopsies (Corse et al., 1996) consistent with demyelinating pathophysiology.

Clinical improvement after high dose intravenous immunoglobulin treatment was observed to be accompanied by either a reduction or total disappearance of PMCB with an increment in proximal and/or distal CMAP amplitude (Chaudhry et al., 1994). The degree of clinical improvement did not always correlate with the degree of reversal of conduction block.

Chronic ataxic neuropathy with benign IgM paraprotein activity against disialosyl gangliosides

There has recently been an increasingly recognised IgM paraproteinaemic syndrome in which IgM reacts with gangliosides bearing NeuNAc( $\alpha 2-8) \operatorname{NeuNAc}(\alpha 2-3)$ Gal-linked disialosyl groups. These include GD1b, GT1a, GT1b, GQ1b and GD3. Some of the IgM paraproteins also show reactivity against the sialated glycoprotein epitopes on human red blood cells: Pr1d or Pr2. Autoantibodies of this specificity are shown to be cytotoxic to rat dorsal root ganglion neurons (Ohsawa et al., 1993).

\section{Clinical features}

llyas et al (1985) first described a patient with a predominantly sensory demyelinating neuropathy. The monoclonal IgM reacted best with GD3 and GT1b but showed strong cross reactivity with GD1b and GD2, all containing disialosyl configurations. Since then 7 other patients with predominantly large fibre sensory neuropathy and monoclonal IgM antibodies to disialosyl groups have been reported (Arai et al., 1992; Duane et al., 1992; Herron et al., 1994; Obi et al., 1992; Oka et al., 1996; Willison et al., 1993; Yuki et al., 1992;). In 2 patients (Arai et al., 1992; Willison et al., 1993), the IgM paraproteins also reacted with antiPr2 and antiPr1d in the one reported by Herron et al (1994). 
Younes-Chennoufi et al (1992) also reported a case of biclonal lgMk with sensorimotor axonal neuropathy with antiGD1b activity. The biclonal IgM paraproteins did not react with GM1, asialo-GM1, GT1b, GD2 or GD3.

Of the 8 patients with monoclonal IgM antidisialosyl activity, 6 were male with ages ranging between 39-77 y (mean $59.3 \mathrm{y}$ ). The duration of symptoms was from 3 mo to $25 \mathrm{y}$ (mean $10 \mathrm{y}$ ). The paraproteins were $\lg M \kappa$ in 4 cases and $\lg M \lambda$ in 4 with serum $\lg M$ at 1.35 to $17.8 \mathrm{~g} / \mathrm{L}$. All patients presented with initial paraesthesiae and/or numbness in distal arms or legs. Unsteadiness especially in the dark was noted early. Only in 1 case (Arai et al., 1992) was tremor one of the presenting symptoms. Cold induced acrocyanosis was noted in 1 of the 3 patients with cold agglutinins. Symptoms were slowly progressive. One patient (Herron et al., 1994) had acute lower limb weakness in association with gastroenteritis and worsening of symptoms with viral infection. On one occasion, he developed transient incomplete ophthalmoplegia, bulbar palsy, dysarthria and dysphagia reminiscent of Miller Fisher syndrome during influenza infection. This latter episode was also complicated by haemolytic anaemia. Another patient (Yuki et al., 1992) had 10 acute relapses of large fibre sensory neuropathy in $15 \mathrm{y}$, usually after upper respiratory tract infection. Although both patients recovered from the acute episodes, there were residual neurological deficits that progressed slowly over the years.

On examination, all patients had prominent large fibre sensory deficits with ataxic gait, marked loss of vibration and joint position sense and positive Romberg's sign. A relatively minor impairment of light touch and pinprick sensation was detected in a glove and stocking distribution. Tendon reflexes were all absent. There was no muscle weakness in 4 cases. Of the other 4 cases, weakness was minimal and confined to or more prominent in the hands. Fluctuating ptosis and 
upward gaze paresis was noted in the first reported case (Arai et al., 1992) even though this did not lead to any symptoms.

As the patients were identified by typical IgM antibody activity, the incidence of antibodies to gangliosides with disialosyl groups was $100 \%$. The acronym of CANOMAD has been suggested by $\mathrm{Dr} \mathrm{H} \mathrm{J}$ Willison (Paparounas et al., 1999; Willison et al., 1996) to described this particular syndrome, i.e. chronic ataxic neuropathy ophthalmoplegia, monoclonal IgM protein, cold agglutinins and disialosyl antibodies.

Neurophysiological findings

In 6 cases all SAPs were absent. In the patient reported by Obi et al (1992), median and ulnar SAPs were absent whilst the sural SAP was present but small. It was absent on follow-up study 3 mo later. Another case (Duane et al., 1992) still retained SAPs in the upper limbs while the sural SAP was absent. Both cases had had symptoms for $1 \mathrm{y}$.

Details on motor studies were rather limited. Two cases reported by Obi et al (1992) and Oka et al (1996) probably had an axonal neuropathy with normal or slightly reduced MCV, normal DML and CMAP. The remaining 5 patients had a predominantly demyelinating neuropathy. In the 2 cases (Arai et al., 1992; Willison et al., 1993) with more information on nerve conduction, these appeared to be asymmetric: Arai et al, MCV: median $=42 \mathrm{~m} / \mathrm{s}$, ulnar $=26 \mathrm{~m} / \mathrm{s}$, tibial $=$ $33 \mathrm{~m} / \mathrm{s}$. Conduction block and temporal dispersion were also noted on stimulation of the tibial nerve at the knee; Willison et al, median DML = $5.2 \mathrm{~ms}, \mathrm{MCV}=38 \mathrm{~m} / \mathrm{s}, \mathrm{F}$ waves $=43 \mathrm{~ms}$; peroneal $\mathrm{DML}=6.9 \mathrm{~ms}, \mathrm{MCV}$ $=34 \mathrm{~m} / \mathrm{s}, \mathrm{F}$ waves $=76 \mathrm{~ms} ;$ tibial $\mathrm{DML}=5.8 \mathrm{~ms}, \mathrm{MCV}=31 \mathrm{~m} / \mathrm{s}, \mathrm{F}$ waves $=84 \mathrm{~ms}$.

Visual evoked potentials performed in the same 2 cases were normal. The blink reflex in the case of Arai et al (1992) showed an afferent defect with symmetrically prolonged $\mathrm{R} 1$. 


\section{Other laboratory results}

Cerebrospinal fluids were acellular with elevated protein levels (0.5-1.7 $\mathrm{g} / \mathrm{L}$ ) in 5 cases. The other 3 cases were normal. Erythrocyte sedimentation rates were performed in 2 cases and these were raised at $96-110 \mathrm{~mm} / \mathrm{h}$.

\section{Chronic sensory neuropathy with benign IgM activity against}

\section{sulphatides}

Sulphatide is a galactocerebroside (galactosyl-ceramide) sulphated in the carbon-3 position. It is a major component of peripheral nerve myelin but is also abundant in the central nervous system. Sulphatides are known to be present in both white and grey matter (Norton, Cammer, 1984). Their wide distribution probably results in several different patterns of IgM binding to neural tissues. In peripheral nerve, the most common targets are axons, resident macrophages and Schwann cell cytoplasm. In the cerebellum, IgM bound to neuronal nuclei, white matter or neuropil in the molecular and granular cell layers (Lopate et al., 1997b). Binding was also found to the surface of the neurons of rat dorsal root ganglia (Quattrini et al., 1992), oligodendroglia, ependymal cells, glial cell process in the periaqueductal grey matter and nucleus interpeduncularis (Zalc et al., 1981). Binding to neuronal nuclei in the cerebellum was usually found in serum that recognised peripheral nerve axons or macrophages (Lopate et al., 1997b).

Raised titres of antisulphatide antibodies have been reported in a variety of conditions: multiple sclerosis (Ryberg, 1978), idiopathic thrombocytopenic purpura (Van Vliet et al., 1987), dermatomyositis (Lopate et al., 1997a) and chronic active hepatitis (Toda et al., 1990). Recently both monoclonal or polyclonal $\lg M$, and $\lg G$ and $\lg A$ antibodies reacting with sulphatides were reported to be associated with predominantly sensory axonal neuropathy, demyelinating neuropathy 
with antiMAG antibodies (llyas et al., 1992; Pestronk et al., 1991) and also in lower motor neuron syndromes (Lopate et al., 1997a) and the Guillain-Barré syndrome (GBS) (Fredman et al., 1991; llyas et al., 1991; Lopate et al., 1997a). In several patients, the antisulphatide crossedreacted with MAG/SGPG (llyas et al., 1986b; van den Berg et al., 1993) and chondroitin sulphate (Nemni et al., 1993).

\section{Clinical features}

Fifteen percent of healthy subjects have been found to have antisulphatide antibodies (Fredman et al., 1991). Antisulphatide antibodies were also found in other neurological conditions in lower titres (van den Berg et al., 1993). Raised antisulphatide therefore depends on the determined upper 'normal' limit and the method used in detecting the antibodies. These probably resulted in variation in the incidence of raised antibodies. Pestronk et al (1991) found elevated antisulphatides in $28 \%$ of patients with neuropathy whilst the incidence was $4.8 \%$ (6 in 125) in the series of van den Berg et al (1993). There were 2 recognisable chronic neuropathy syndromes in patients with antibodies to sulphatides: one resembled ganglioneuritis and the other, small fibre neuropathy (Latov, 1995; Pestronk et al., 1991; van den Berg et al., 1993).

Of the 15 patients with benign IgM paraproteinaemic neuropathy and high titres of antisulphatide antibodies of more than 4500 (2 from Pestronk et al in 1991, 1 from van den Berg et al in 1993, 3 from NobileOrazio et al in 1994 and 9 from Lopate et al in 1997a), there were 10 males and 5 females. The mean age of onset was $63.5 \mathrm{y}$, ranging from 46-82 y with a duration of symptoms of 1-23 y. The paraprotein was $\lg M_{\kappa}$ in 10 and $\lg M \lambda$ in 3 . The type of light chain was not mentioned in 2. The majority of the antisulphatide antibodies were concordant with the $M$ proteins but the light chain could be either $\mathrm{k}$ or $\lambda$ or both. Prominent pain or dysaesthesia was reported in 2 out of the 10 cases. 
Although a few patients had only sensory symptoms, there was usually a mild but detectable motor weakness on examination. This was usually distal and symmetrical. Small as well as large sensory fibres were involved on clinical testing. There was no thickening of peripheral nerves or cranial nerve involvement.

In recent study by Lopate et al (1997a) on 19 patients with antisulphatide antibodies, it was suggested that patients with $\mathrm{M}$ proteins are more likely to have motor abnormalities, no pain and a demyelinating neuropathy whereas those without an $M$ protein commonly have neuropathic pain, no significant weakness and an axonal neuropathy.

\section{Neurophysiology}

In cases with clinical features of sensory neuropathy, the neurophysiological findings were predominantly demyelinating. The ones with small fibre neuropathy showed no abnormalities in the peripheral nerve conduction studies or ,apparently, on nerve biopsy.

\section{Neuropathy with benign $\operatorname{Ig} M$ reacting with chondroitin sulphate}

Chondroitin sulphates (ChS) are sulphated glycosaminoglycans, hexamine containing complex polysaccharides, which are major constituents of connective tissues. In peripheral nerves, immunocytochemical studies show the presence of proteoglycans in the Schwann cell basal lamina of the sciatic nerve as well as in the extracellular matrix surrounding the collagen fibrils (Aquino et al., 1984a; Aquino et al., 1984b).

A progressive sensorimotor neuropathy associated with epidermolysis has been described in patients with IgM paraproteins with activity against ChS (Sherman et al., 1983). Several other cases were observed with monoclonal IgM gammopathy in which the neuropathy was predominantly sensory and axonal (Mamoli et al., 1992; Nemni et al., 1993; Nobile-Orazio et al., 1994; Quattrini et al., 1991; Yee et al., 
1989). Endoneurial deposits of $\lg M$ were demonstrated by immunofluorescence microscopy of nerve biopsies of several patients (Latov, 1994; Nemni et al., 1993; Takatsu et al., 1985). The latter suggested that anti-ChS might have a role in neuropathy. Briani et al (1998) in their application of a new sensitive and reliable assay for detection of chondroitin sulphate $C$ to 480 sera, found 11 patients with elevated titres of 12,800 or above. Seven patients had sensory axonal neuropathy, 3 amyotrophic lateral sclerosis and 1 corticobasal degeneration. Thus although anti-ChS antibodies occur in sensory axonal neuropathy, they also occur in other diseases.

\section{Other IgM antibody activities}

There has been a recent report of antibodies to heparan sulphate glycosaminoglycans in approximately one third of patients with demyelinating GBS and in some with chronic inflammatory neuropathies (Pestronk et al., 1998). High levels of monoclonal IgM antibodies to intracellular proteins such as tubulin (Connolly et al., 1993; Connolly, Pestronk, 1997) and intermediate filaments (Dellagi et al., 1982) have also been reported in neuropathy. Connolly et al (1997) suggested a stronger association of anti-tubulin with demyelinating neuropathy if anti-tubulin antibodies recognised the 301 to 304 amino acid epitope on tubulin.

In some patients with peripheral neuropathy with benign IgM paraproteinaemia, no demonstrated autoantibodies were detected. The antibodies in these patients may be unrelated to the neuropathy or had activity against unidentified peripheral nerve components.

\subsection{Benign IgG or IgA or IgD paraproteinaemic neuropathy}

Neuropathy associated with IgG paraproteins is relatively uncommon in comparison with IgM despite their commoner occurrence in the general population. Some of the benign lgG paraproteinaemic neuropathy cases have been discussed in section 1.3. Gosselin et al (1991) and 
Yeung et al (1991) in their studies of 24 out of 65 and 11 out of 62 patients did not find any difference between $\lg G$ and $\operatorname{lgM}$ paraproteinaemic neuropathy whilst subsequent studies by NobileOrazio et al (1992), Suarez and Kelly (1993), Notermans et al (1994) and Simovic et al (1998) did.

Notermans et al (1994) suggested dividing the patients into those with an axonal neuropathy and those with a demyelinating neuropathy. In patients with an axonal neuropathy, the neuropathy is slowly progressive, distal, symmetrical and sensory or sensorimotor, resembling chronic idiopathic axonal polyneuropathy. The presence of monoclonal gammopathy in this instance may be an epiphenomenon. In some patients, the $M$ proteins were found in sural nerve biopsies (Bosch et al., 1982; Dalakas, Engel, 1981; Sewell et al., 1981) and in 2 of them reactivity of IgG paraproteins has been reported against a neurofilament protein (Nemni et al., 1990; Sewell et al., 1981). These findings together with the improvement of neuropathy after immunosuppressive therapy or plasma exchange or both (Bosch et al., 1982; Dalakas, Engel, 1981; Dyck et al., 1991; Fineman, McKendell, 1990) suggest that at least in some patients the paraprotein may play a pathogenetic role in the neuropathy. This group of patients certainly demonstrates a variegated clinical picture.

Neuropathy associated with IgA paraproteins are less common and less well characterised. Cases were reported by Bailey et al (1986), Bosch et al (1982), Hemachudha et al (1989), Gosselin et al (1991) and Yeung et al (1991). A recent study by Simmons et al (1993) recorded 5 patients with benign IgA paraproteinaemic neuropathy with 1 patient being diabetic. They found varied clinical presentations, electrophysiological and histological findings and concluded that benign IgA paraproteinaemic neuropathy is heterogeneous. The numbers of recorded cases so far are insufficient for any general comment.

To date, only a single case of $\lg D \lambda$ benign paraproteinaemic neuropathy was reported (Hansen et al., 1989). The patient presented 
with chronic progressive distal sensorimotor neuropathy with prominent ataxia at the age of 71 . Neurophysiological and pathological findings were said to be consistent with an axonal degeneration. The lg did not show reactivity to myelin antigens and no ig or complement deposits were found on sural nerve biopsy.

\subsection{Neuropathology of benign paraproteinaemic neuropathy}

The most characteristic pathological change in the peripheral nerves in patients with benign IgM paraproteinaemic neuropathy is chronic demyelination and remyelination with varying degree of axonal loss (Smith et al., 1983; Vital et al., 1989; Yeung et al., 1991). Hypertrophic change ("onion bulb" formation) may be seen and although inflammatory cells have been reported in a few cases (Forssman et al., 1973; Vital et al., 1985), they are usually absent. Deposition of IgM is frequently demonstrated on surviving myelin sheaths (Abrams et al., 1982; Kahn et al., 1981; Leibowitz et al., 1983; Meier et al., 1984b; Smith et al., 1983), more frequently in IgM with a $\mathrm{\kappa}$ light chain (Thomas, Willison, 1994; Yeung et al., 1991). Although the demyelination is complement-mediated (Monaco et al., 1990), routine examination for complement is usually not performed and therefore the true incidence is not known.

The salient feature is the electron microscopic appearance of abnormally widely spaced myelin. This phenomenon was first recognised in patients with Waldenström macroglobulinaemia (Melmed et al., 1983) and subsequently shown to be a frequent finding (approximately $50 \%$ ) in benign IgM monoclonal gammopathy (Pollard et al., 1985; Smith et al., 1983; Steck et al., 1987; Trojaborg et al., 1995; Vital et al., 1989). Vital et al (1989) considered the occurrence of widely spaced myelin to be correlated strongly with IgM paraproteins with antiMAG activity. Vallat et al (1996) also found a high incidence (10 out 16 cases) of patients with benign monoclonal gammopathy with IgM activity against SGPG to have positive direct immunofluorescence and 
widely spaced myelin. Unfortunately antiMAG determination was not performed. AntiMAG usually cross reacts with SGPG (llyas et al., 1992).

Widely spaced myelin has occasionally been described in IgG paraproteinaemia (Powell et al., 1984; Vital et al., 1989), Guillain-Barré syndrome (Vallat et al., 1994), CIDP (Vital et al., 1986) and in experimental allergic neuritis (King, Thomas, 1984). The finding is therefore not pathognomonic for either benign IgM or malignant IgM paraproteinaemia.

The increase in the periodicity is due to separation between the lamellae at the intermediate lines, i.e. it involves an expanded extracellular space. The intermediate lines are at a normal distance from the major dense lines which are widely separated, producing a periodicity of approximately 2.5 times normal. This differs from uncompacted myelin in which there are no major dense lines and Schwann cell cytoplasm is still evident. The abnormal periodicity affects both remyelinating fibres as well as atrophic fibres and fibres that are not undergoing demyelination or remyelination. The outermost lamellae are most frequently affected. The inner lamellae are only affected when fibres are remyelinating (Jacobs, Scadding, 1990). The distribution of widened lamellae frequently includes the MAG distribution areas, i.e. paranodes, Schmidt-Lanterman incisures, periaxonal region and mesaxons but other locations not corresponding to recognised sites for MAG are also involved, i.e. outermost lamellae of compact myelin. The change at the latter site is thought to be due to cross reactivity of the antiMAG antibodies with other glycoconjugates, Po, PMP22 and SGPG. The incidence of widely spaced myelin varies greatly from one case to the other and may affect 6\%-90\% (Jacobs, Scadding, 1990; Jacobs, 1996; King, Thomas, 1984; Mendell et al., 1985) of myelinated fibres. In some instances, the low reported incidence may be the result of failure of detection. 
Widely spaced myelin has been attributed to the direct binding of the IgM paraprotein to the myelin membrane (Jacobs, Scadding, 1990; Latov et al., 1988; Meier et al., 1984b; Yu, Ariga, 1998) as a result of the attractive force between their cationic properties and the strong fixed negative charge on the external surface of Schwann cell. This has been thought to produce a change in permeability which leads to myelin destruction. As similar ultrastructural changes can be found in other conditions not associated with the presence of antimyelin antibodies, intramyelinic oedema has been suggested as an alternative mechanism on the basis of the experimental reproduction of the pathology by changes in osmotic pressure without exposure to lg (King, Thomas, 1984).

Other changes in IgM paraproteinaemic neuropathy include tomacula which consist of focal areas of hypermyelination with redundant folds of myelin especially on the external aspects of the sheath (Rebai et al., 1989; Vital et al., 1985; Vital et al., 1989); the less specific uncompacted myelin lamellae which may occur in the outer region of the sheath (Jacobs, Gregory, 1991; King, 1999b); deposition of highly organised granular material within the widely spaced myelin (Jacobs, Scadding, 1990); occasional deposition of IgM in the endoneurium (Fitting et al., 1979; Vital et al., 1989); and microangiopathy with endothelial cells expanded by microfilaments and thickened basement membrane around endoneurial capillaries by multiple reduplicated layers of basal lamina (Powell et al., 1984). Unmyelinated fibres are not extensively involved but this has not been examined in detail.

Not all patients with benign monoclonal IgM gammopathy and peripheral neuropathy have chronic demyelination. Occasional instances of an axonal neuropathy have been reported (Yeung et al., 1991). This confirms the clinical impression that patients with benign IgM paraproteins, as a whole, are heterogeneous (Yeung et al., 1991). 
As patients with benign IgG paraproteinaemic neuropathy can be categorised into those with axonal and demyelinating neuropathy (Notermans et al., 1996), neuropathological changes therefore reflect the neurophysiological findings. Axonal degeneration was found in 2 out of 6 cases with IgG paraproteinaemia reported by Yeung et al (1991) whilst the rest showed mixed changes of axonal degeneration and demyelination. Sural nerve biopsies of all 5 cases of Bleasel et al (1993) revealed a demyelinating neuropathy with inflammatory $T$ lymphocyte infiltrates resembling CIDP. No immunoglobulin deposition has been demonstrated and widely spaced myelin is rarely found (Powell et al., 1984; Vital et al., 1989). Microtubular structures, staining with IgG antibody and resembling immunotactoid, were observed in the endoneurium, lumina of vasa nervorum and dermis of a patient with benign IgG paraproteinaemic neuropathy (Moorhouse et al., 1992).

Neuropathy associated IgA is less common and the number of recorded cases with nerve biopsy is so far insufficient for any statement to be made.

\subsection{Long term outcome for incidence of haematological malignancy}

Very few studies have been performed on long term follow-up of benign paraproteinaemic neuropathy. Notermans et al (1994), in their unique 5 $y$ follow-up of 32 such patients without treatment, found only 3 patients who developed non-Hodgkin lymphoma. Two had IgMk whilst the other had IgGk paraproteins. Their neuropathy deteriorated acutely. Clinical and laboratory examination was normal including the absence of a sudden rise in the paraprotein level. The diagnoses were made on the finding of abnormal lymphocytes $>30 \%$ on repeated bone marrow investigations.

None of the 18 patients with IgM paraproteinaemic neuropathy (mean $7.4 y$, range 1-14 y) who were followed by Smith in 1994 
developed haematological malignancy. Only 2 had small cell carcinoma of the lung and carcinoma of the pancreas, which could possibly have been related to treatment with cytotoxic drugs.

Seven out of 33 patients with peripheral neuropathy associated with benign IgM antiMAG gammopathy studied by Ellie et al (1996) died during the mean follow-up of 54 mo (range 15-160 mo; in 11 patients the duration exceeded $60 \mathrm{mo}$ ). Only 2 developed malignancy. One had a fatal B-cell cerebral lymphoma with intrathecal secretion of the paraprotein of the same heavy and light chain as the serum monoclonal component (Ellie et al., 1995). This was not preceded by deterioration in the peripheral neuropathy. The other died from pancreatic malignancy.

The incidence of haematological malignancy for patients with benign paraproteinaemic neuropathy therefore ranges from $0-9.4 \%$ at a mean follow-up of $5 \mathrm{y}$. As there is clear evidence of an increased incidence of malignancy with time, follow-up over a longer period to assess the outcome is thus desirable.

\subsection{Aims of present study}

Although a considerable amount of information about neuropathy associated with benign monoclonal gammopathy has accumulated since its first recognition a quarter of a century ago, the foregoing review has indicated that a number of unanswered questions still remain. The most important of these are (1) the relationship of the paraprotein to the neuropathy, i.e. is the paraprotein causal or an epiphenomenon; (2) the correlation of the clinical manifestations with the type of paraprotein and the identification of different nosological entities; (3) the response of different syndromes to treatment; and (4) the long term outlook, including the incidence of haematological malignancy. The aim of the present study was to determine the long term clinical outcome in a group of patients initially diagnosed as having neuropathy associated with a benign paraprotein (MGUS), including 
changes in the electrodiagnostic findings over time, and to assess the risk of developing haematological malignancy. An attempt will also be made to identify individual nosological subgroups. As the cases collected were under the care of a large number of different clinicians, no detailed conclusions with respect to treatment was possible. The important question of the relationship of the paraprotein to the neuropathy is a matter of experimental investigation rather than clinical analysis. 
Table 1.1 Properties of human immunoglobulins

\begin{tabular}{|c|c|c|c|c|c|}
\hline & IgG & IgA & IgM & IgD & IgE \\
\hline Heavy chain & $\gamma$ & $\alpha$ & $\mu$ & $\delta$ & $\varepsilon$ \\
\hline $\begin{array}{l}\text { Heavy chain } \\
\text { subclass }\end{array}$ & $\gamma_{1}, \gamma_{2}, \gamma_{3}, \gamma_{4}$ & $\alpha_{1}, \alpha_{2}$ & & & \\
\hline $\begin{array}{l}\text { Type of light }(\mathrm{L}) \\
\text { chain }\end{array}$ & $\kappa$ and $\lambda$ & $\kappa$ and $\lambda$ & $\kappa$ and $\lambda$ & $\kappa$ and $\lambda$ & $\kappa$ and $\lambda$ \\
\hline $\begin{array}{l}\text { Molecular } \\
\text { formula }\end{array}$ & $\gamma_{2} \mathrm{~L}_{2}$ & $\begin{array}{l}\alpha_{2} \mathrm{~L}_{2} \\
\left(\alpha_{2} \mathrm{~L}_{2}\right)_{2} \mathrm{SC} / \mathrm{J}\end{array}$ & $\left(\mu_{2} \mathrm{~L}_{2)} \mathrm{s} \mathrm{J}\right.$ & $\delta_{2} L_{2}$ & $\varepsilon_{2} \mathrm{~L}_{2}$ \\
\hline $\begin{array}{l}\text { Molecular weigh } \\
\left(\times 10^{3}\right)\end{array}$ & $146-170$ & 160 & 970 & 184 & 188 \\
\hline $\begin{array}{l}\text { Sedimentation } \\
\text { constant }\end{array}$ & $7 \mathrm{~s}$ & $\begin{array}{l}7 \mathrm{~s} \\
11 \mathrm{~s} \text { for secretory IgA }\end{array}$ & $19 \mathrm{~s}$ & $7 \mathrm{~s}$ & $8 s$ \\
\hline $\begin{array}{l}\text { Mean serum } \\
\text { concentration } \\
(\mathrm{mg} / \mathrm{ml})\end{array}$ & $0.5-9$ & 3.0 & 1.5 & $0.05-0.5$ & 0.00005 \\
\hline $\begin{array}{l}\text { Mean serum } \\
\text { half life (days) }\end{array}$ & $7-21$ & $\begin{array}{l}6 \\
\text { ?in secretory IgA }\end{array}$ & 10 & 3 & 2 \\
\hline $\begin{array}{l}\% \text { intravascular } \\
\text { distribution }\end{array}$ & 45 & $\begin{array}{l}42 \\
\text { trace for secretory IgA }\end{array}$ & 80 & 75 & 50 \\
\hline
\end{tabular}

SC: secretory component; J: J chain.

Adapted from Fig 6.4 in Antibodies and their receptors. Turner, M., Immunology, Riott I, Brostoff J, Male D (Eds.). Immunology, $5^{\text {th }}$ edn. London: Mosby International, 1998, Chapter 6, p 73. 
Table 1.2 Ganglioside structure

\begin{tabular}{|c|c|}
\hline Name & Carbohydrate sequence \\
\hline $\mathrm{GM}_{1}$ & $\begin{array}{c}\operatorname{Gal}(\beta 1-3) \text { GalNAc }(\beta 1-4) \mathrm{Gal}(\beta 1-4) \mathrm{Glc}(\beta 1-1) \text { Ceramide } \\
(\alpha 2-3) \\
\text { NeuNAc }\end{array}$ \\
\hline Asialo- $\mathrm{GM}_{1}\left(\mathrm{GA}_{1}\right)$ & $\operatorname{Gal}(\beta 1-3) \operatorname{GalNAc}(\beta 1-4) \mathrm{Gal}(\beta 1-4) \mathrm{Glc}(\beta 1-1)$ Ceramide \\
\hline GM2 & $\begin{array}{l}\text { GalNAc }(\beta 1-4) \mathrm{Gal}(\beta 1-4) \mathrm{Glc}(\beta 1-1) \text { Ceramide } \\
(\alpha 2-3) \\
\text { NeuNAc }\end{array}$ \\
\hline $\mathrm{GD}_{\mathrm{la}}$ & $\begin{array}{ll}\mathrm{Gal}(\beta 1-3) \mathrm{GalNAc}(\beta 1-4) \mathrm{Gal}(\beta 1-4) \mathrm{Glc}(\beta 1-1) \text { Ceramide } \\
(\alpha 2-3) & (\alpha 2-3) \\
\text { NeuNAc } & \text { NeuNAc }\end{array}$ \\
\hline $\mathrm{GD}_{1 \mathrm{~b}}$ & $\begin{aligned} \mathrm{Gal}(\beta 1-3) \mathrm{GalNAc}(\beta 1-4) \mathrm{Gal}(\beta 1-4) \mathrm{Glc}(\beta 1-1) \text { Ceramide } \\
\\
(\alpha 2-3) \\
\text { NeuNAc } \\
(\alpha 2-8) \\
\text { NeuNAc }\end{aligned}$ \\
\hline $\mathrm{GD}_{2}$ & $\begin{array}{l}\text { GalNAc }(\beta 1-4) \mathrm{Gal}(\beta 1-4) \mathrm{Glc}(\beta 1-1) \text { Ceramide } \\
(\alpha 2-3) \\
\text { NeuNAc } \\
(\alpha 2-8) \\
\text { NeuNAc }\end{array}$ \\
\hline $\mathrm{GD}_{3}$ & $\begin{array}{l}\text { Gal }(\beta 1-4) \mathrm{Glc}(\beta 1-1) \text { Ceramide } \\
(\alpha 2-3) \\
\text { NeuNAc } \\
(\alpha 2-8) \\
\quad \text { NeuNAc }\end{array}$ \\
\hline $\mathrm{GT}_{1 \mathrm{~b}}$ & $\begin{array}{ll}\operatorname{Gal}(\beta 1-3) \mathrm{GalNAc}(\beta 1-4) \mathrm{Gal}(\beta 1-4) \mathrm{Glc}(\beta 1-1) \text { Ceramide } \\
(\alpha 2-3) & (\alpha 2-3) \\
\text { NeuNAc } & \text { NeuNAc } \\
& (\alpha 2-8) \\
& \text { NeuNAc }\end{array}$ \\
\hline $\mathrm{GT}_{12}$ & $\begin{array}{ll}\mathrm{Ga}(\beta 1-3) \mathrm{GalNAc}(\beta 1-4) \mathrm{Gal}(\beta 1-4) \mathrm{Glc}(\beta 1-1) \text { Ceramide } \\
(\alpha 2-3) & (\alpha 2-3) \\
\text { NeuNAc } & \text { NeuNAc } \\
(\alpha 2-8) & \\
\text { NeuNAc } & \end{array}$ \\
\hline $\mathrm{GQ}_{\mathrm{lb}}$ & $\begin{array}{ll}\text { Gal }(\beta 1-3) \text { GalNAc }(\beta 1-4) \mathrm{Gal}(\beta 1-4) \mathrm{Glc}(\beta 1-1) \text { Ceramide } \\
(\alpha 2-3) & (\alpha 2-3) \\
\text { NeuNAc } & \text { NeuNAc } \\
(\alpha 2-8) & (\alpha 2-8) \\
\text { NeuNAc } & \text { NeuNAc }\end{array}$ \\
\hline $\mathrm{LM}_{1}(\mathrm{SPG})$ & $\begin{array}{l}\text { Gal( } \beta 1-3) \text { GalNAc( } \beta 1-4) \mathrm{Gal}(\beta 1-4) \mathrm{Glc}(\beta 1-1) \text { Ceramide } \\
(\alpha 2-3) \\
\text { NeuNAc }\end{array}$ \\
\hline
\end{tabular}




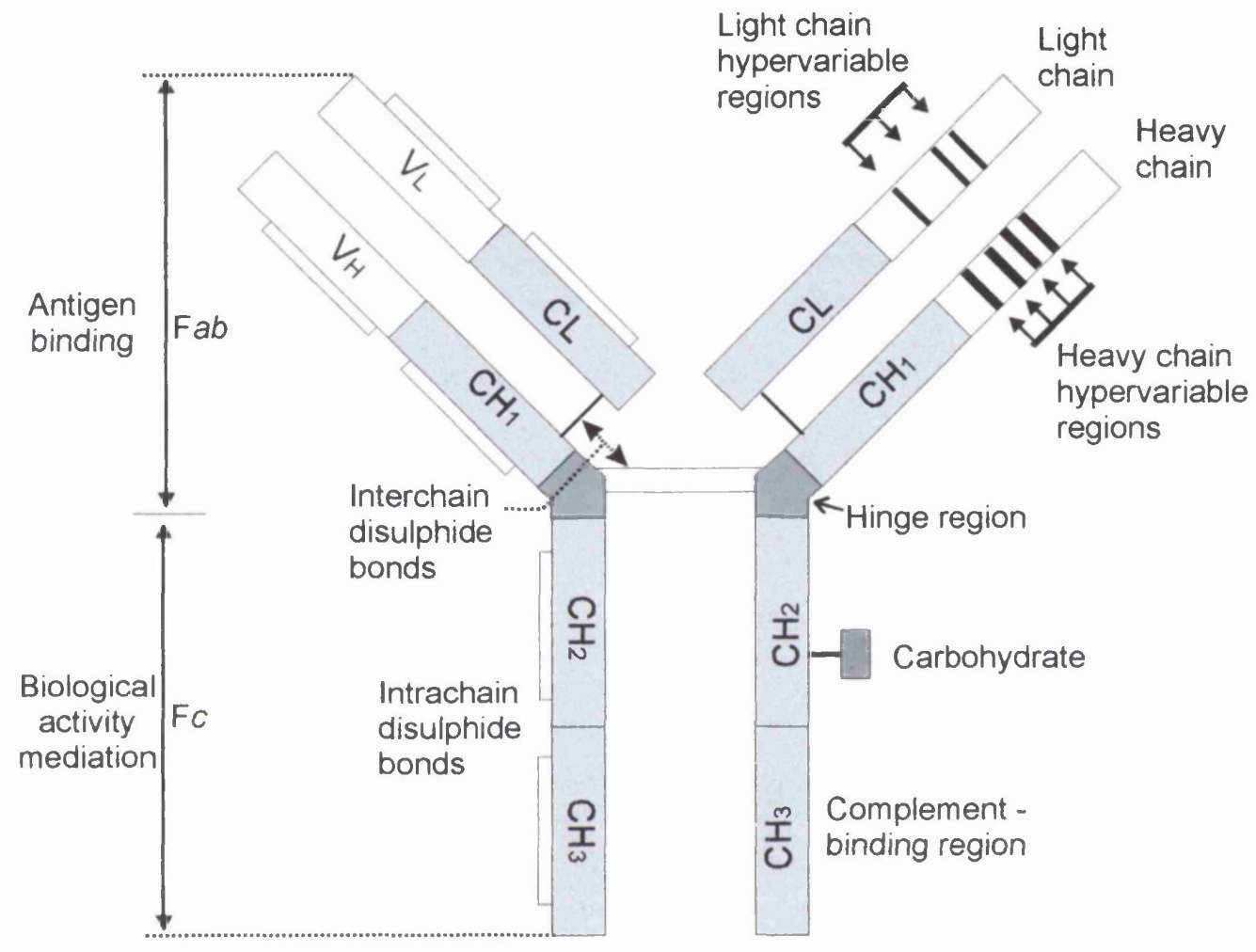

$V_{L}$ and $V_{H}$ : Variable regions

$\mathrm{CL}$ and $\mathrm{CH}_{\mathrm{H}}$ : Constant regions

Figure 1.1 Structure of the Ig molecule. A schematic representation of an IgG molecule indicating the chain and domain structure of the molecule and the existence of hypervariable regions within variable regions of both $\mathrm{H}$ and $\mathrm{L}$ chains. Fab and $\mathrm{Fc}$ refer to fragments of the IgG molecule formed by papain cleavage at the hinge region ( at residue 224 ) yielding 2 Fab fragments and an Fc fragment.

Taken from Figure 417-1. Paul,WE, The immune system. In Wyngaarden JB, Smith LH Jr. editors. Cecil Text book of Medicine, 18th edn. Philadelphia: Saunders WB; 1988 , p 1933 


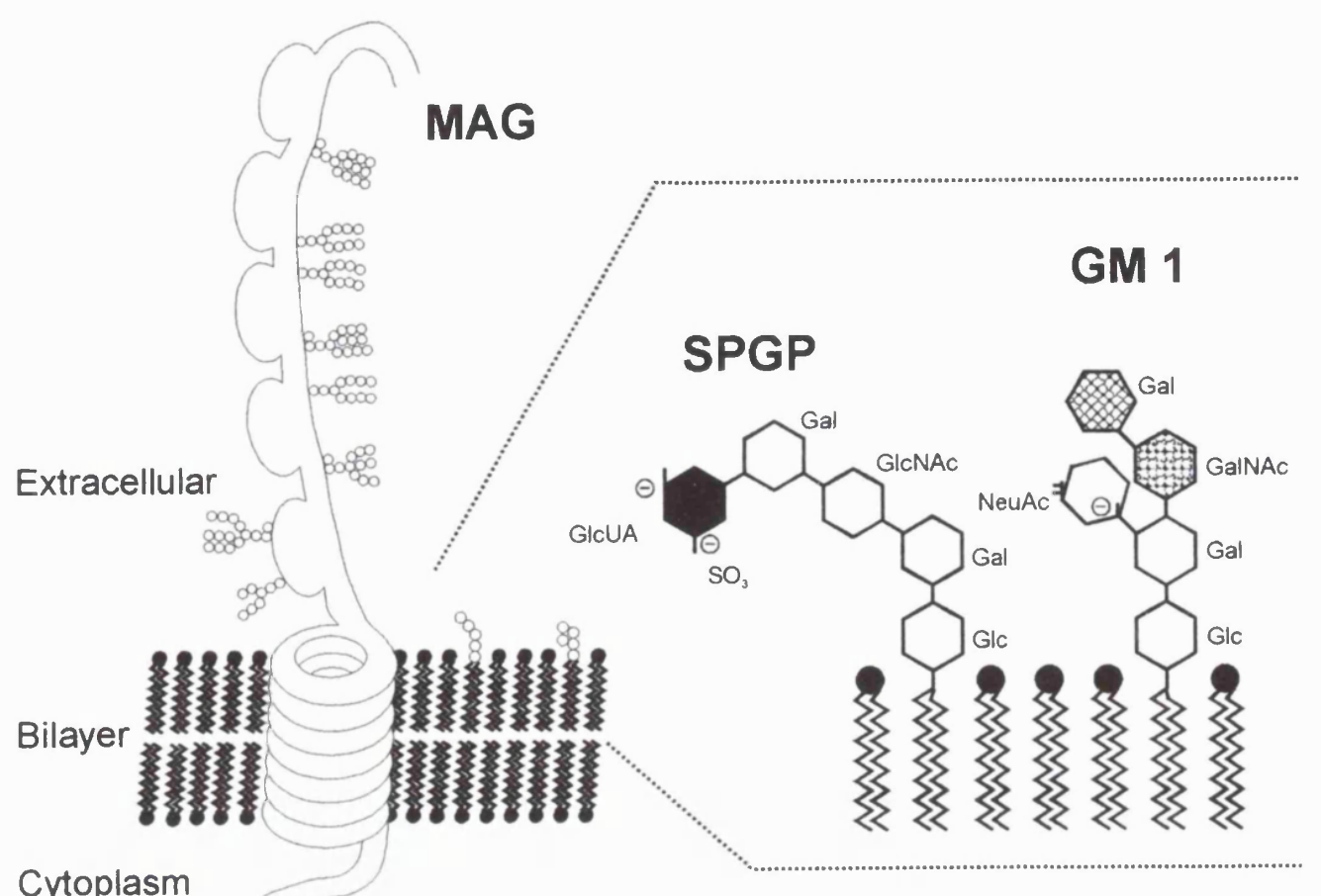

Cytoplasm

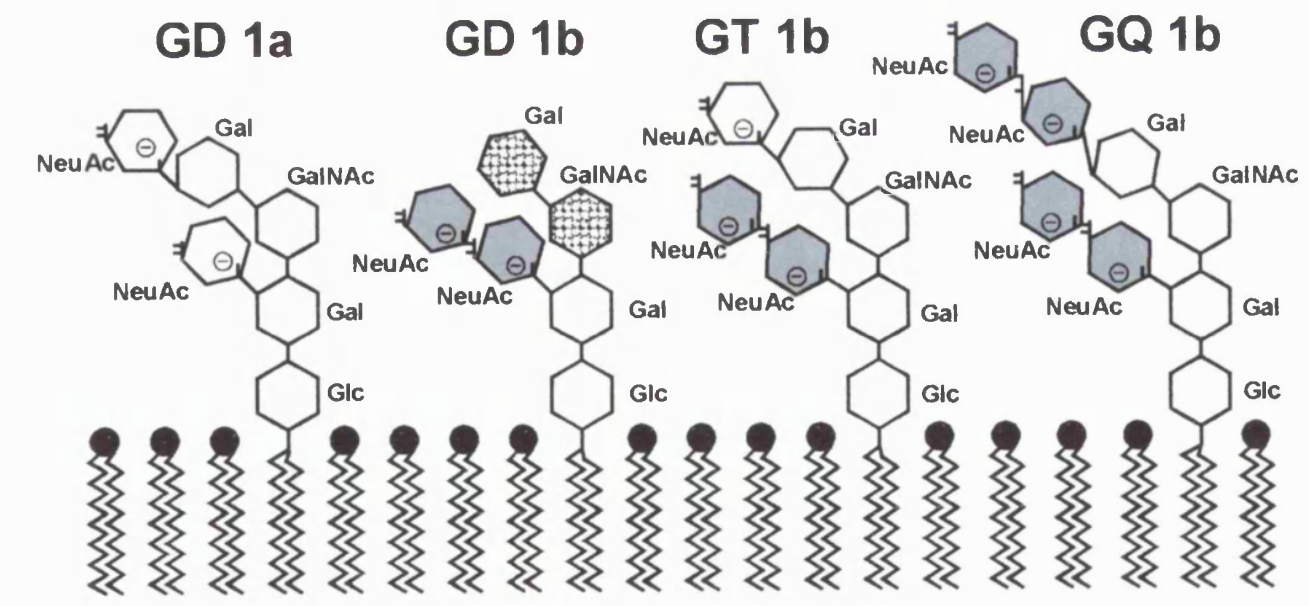

Figure 1.2 Schematic representations of some glycoconjugates that have been implicated as antigens in peripheral neuropathies.

(Taken from Fig 1 in Quarles and Weiss, Muscle Nerve 1999;22:800-822) 


\title{
CHAPTER 2 \\ PATIENTS AND METHODS
}

\subsection{Patients}

\author{
Recruitment \\ Inclusion criteria
}

\subsection{Methods}

Clinical assessment

Neurophysiology

Criteria for the diagnosis of demyelinating neuropathy

Criteria for the diagnosis of axonal degeneration

Immunological investigations

Assays for antimyelin associated glycoprotein antibody

Ganglioside antibody assays

LM1

Immunoabsorption and purification for cold agglutinating antibody

Histopathological investigations

Statistical methods 


\section{PATIENTS AND METHODS}

\subsection{Patients}

\section{Recruitment}

Three hundred and thirty patients were identified under the National Hospital diagnostic indices (before 1994: 357.3-, 357.80-Neuropathy, inflammatory and toxic. After 1994: G609-Hereditary and idiopathic neuropathy; G 628 and G 629-Other polyneuropathies; D892 and D891 -Other disorders involving the immune mechanism NEC). Additional patients were obtained from referrals to Professor $P K$ Thomas and to the Peripheral Nerve Clinics at the National Hospital for Neurology and Neurosurgery and the Royal Free Hospital. Their case notes were reviewed. Fifty-two patients had peripheral neuropathy associated with benign monoclonal gammopathy. Two patients refused to participate therefore leaving 50. An additional 5 patients with chronic inflammatory demyelinating polyneuropathy (CIDP) were included in the study. The latter had no gammopathy on repeat blood testing.

\section{Inclusion criteria}

All patients included had a peripheral neuropathy on clinical and electrophysiological examination. All patients, apart from the 5 with CIDP, had paraproteinaemia, no immunosuppression, normal bone marrow biopsy (normal cellularity with $<10 \%$ of plasma cells and $<30 \%$ lymphoid cells), normal skeletal X-ray survey and no evidence of Bence-Jones proteinuria. Other causes of neuropathy were excluded by physical examination and screening tests (complete blood count, erythrocyte sedimentation rate, random blood glucose, urea and electrolytes, liver function tests, thyroid function tests, serum vitamin $\mathrm{B} 12$ and folate concentrations, chest $\mathrm{X}$-ray). Monoclonal or bi/triclonal gammopathy was detected by agarose gel serum electrophoresis. Immunoelectrophoresis was performed using monospecific sera to $\operatorname{lgM}$, 
IgG and IgA heavy chains and $\mathrm{k}$ and $\lambda$ light chains. If the results of the immunoelectrophoresis were equivocal, immunofixation with monospecific sera was performed. Immunoglobulin was quantified by densitometry. Patients with CIDP satisfied clinical, neurophysiological, pathological and CSF criteria (Ad Hoc Subcommittee of the American Academy of Neurology AIDS Task Force, 1991). All patients had had the illness for more than $5 \mathrm{y}$ from the onset of symptoms and gave informed consent prior to participation.

\subsection{Methods}

\section{Clinical assessment}

Proforma I was used after reviewing the case notes whereas Proforma II was used when the patients themselves were reviewed. For patients who died, follow-up information was obtained by telephone contact or by questionnaires to the relatives or general practitioners. Three patients had to be traced through the Office for National Statistics.

Symptoms were then scored using the neurological symptom score described by Dyck et al (1980). Scoring for signs: muscle strength (MRC grading), tendon reflexes, sensory function including touch, pinprick, vibration and joint position sense, was performed as described Notermans et al (1994). Scoring for clinical disability employed the rating scale given by Hughes et al (1978).

Proforma I and II, clinical scoring and questionnaires were included at the end of the chapter.

\section{Neurophysiological assessment}

All patients underwent standard nerve conduction studies (NCS). These were performed using Neuromax EMG system (Excel Tech Limited, Ontario, Canada). Supramaximal stimulation was delivered at $1 / \mathrm{s}$ at $0.2-1.0 \mathrm{~ms}$ pulse width via surface electrodes (Medelec 16893, Woking, UK). Recording was by surface electrodes (Medelec 16933, Woking, UK). The low frequency filter was 30 and $5 \mathrm{~Hz}$ for the sensory 
and motor studies respectively whilst the high frequency filter was 2000 $\mathrm{Hz}$, the same for both. Skin temperature was maintained at $32^{\circ} \mathrm{C}$. Electromyography, when performed, was by concentric needle electrodes (N53156, N53153, Oxford Instruments, Abingdon, UK). Motor NCS and F wave recordings were carried out for the median and/or ulnar, common peroneal and tibial nerves. Sensory nerve action potentials were examined for the median, ulnar, radial and sural nerves, the latter 2 with an antidromic technique. Ring electrodes (Medelec 16639, Woking, UK) were used for median and ulnar stimulation over the index and fifth fingers. Latency was measured for the onset to positive deflection and amplitude from peak to peak measurement.

Criteria for an electrophysiological diagnosis of demyelinating neuropathy:

1. Reduction of MCV in 2 or more nerves to $<70 \%$ of normal ( 35 $\mathrm{m} / \mathrm{s}$ in the arms and $29 \mathrm{~m} / \mathrm{s}$ in the legs) (Ad Hoc Subcommittee of the American Academy of Neurology AIDS Task Force, 1991)

2. Prolonged DMLs in 2 or more nerves $>150 \%$ (median $>5.4 \mathrm{~ms}$, ulnar $>3.8 \mathrm{~ms}$, common peroneal $>7.2 \mathrm{~ms}$, tibial $>7.6 \mathrm{~ms}$ ) (Ad Hoc Subcommittee of the American Academy of Neurology AIDS Task Force, 1991)

3. Partial conduction block or temporal dispersion in 1 or more nerves at sites not prone to compression (Oh, 1993b).

- peak to peak amplitude criterion, if distal-proximal amplitude $>25 \%$ for median, ulnar and peroneal nerves and $>36 \%$ for tibial nerve.

- Duration criterion, if the distal compound muscle potentials (CMAP) total duration $>31 \mathrm{~ms}$, proximal CMAP total duration $>35 \mathrm{~ms}$, proximal-distal difference of duration $>\mathbf{2 7} \mathrm{ms}$ for median, ulnar and peroneal nerves and $>33 \mathrm{~ms}$ for tibial nerve.

4. Absent $F$ waves or prolonged minimal $F$ wave latencies in 2 or more nerves of $>150 \%$. 
Criteria for an electrophysiological diagnosis of axonal degeneration :

1. CMAP amplitude reduction without temporal dispersion

2. Reduction of MCV by not more than $30 \%$ normal.

3. Normal MCV in the presence of denervation potentials on electromyographic examination.

4. Absent or low amplitude SAPs without dispersion of the above

The terminal latency index (TLI) was calculated from the terminal distance $(\mathrm{mm})$ divided by conduction velocity $(\mathrm{m} / \mathrm{s}) \times$ terminal latency (ms) and residual latency (RL) from TL-distal distance $(\mathrm{mm})$ I $M C V$ in the forearm $(\mathrm{m} / \mathrm{s})$.

\section{Immunological investigations}

Assay for antimyelin associated glycoprotein (antiMAG) activity

Testing was repeated for paraprotein concentrations and for antiMAG and antiglycolipid antibodies. MAG immunoreactivity was identified in Western blots using equine brain myelin delipidated in ether/ethanol (3:2). Protein concentrations were measured by the method of Lowry et al (1951). Proteins were electrophoretically transferred by the method of Towbin et al (1979) onto nitrocellulose (Schleicher and Schuell, Keene, $\mathrm{NH}$ ). The nitrocellulose blots were air dried and stored in the dark prior to immune staining.

\section{Ganglioside antibody assays}

GM1, GM2, GD1a, GD1b, GT1b, GD3, asialo-GM1. Polystyrene multiwell plates (Immulon 2, Dynatech Laboratories, Ashford, UK) were coated with the purified gangliosides or glycolipids GM1, GM2, GM3, GD1a, GD1b, GT1b, GD3, asialo-GM1 (Sigma Chemical Co., Poole, UK), and GD1b (Calbiochem, Nottingham, UK) by the evaporation at room temperature of $200 \mathrm{ng}$ glycolipid in $100 \mu \mathrm{l}$ of methanol per well. GT1b (Sigma) was noted to contain trace amounts of GD1b. Wells 
were filled with $200 \mu$ phosphate buffered saline (PBS-BSA: pH 7.4, 3\% bovine serum albumin) and left for $2 \mathrm{~h}$ at $4^{\circ} \mathrm{C}$, emptied and $100 \mu \mathrm{l}$ of serially diluted test serum in PBS $0.1 \%$ BSA was added in duplicate to glycolipid coated and to blank wells which had been soaked in methanol and blocked in the same way. The plate was left to incubate with serum overnight at $4^{\circ} \mathrm{C}$, washed 10 times in PBS, and $100 \mu$ l of $1 / 3000$ dilution of peroxidase conjugated rabbit antihuman IgM or IgG (Dako, High Wycombe, UK) or a $1 / 2000$ dilution of antihuman $\kappa$ or $\lambda$ light chain antibody (Sigma) was added. The plate was incubated for a further $4 \mathrm{~h}$ at $4^{\circ} \mathrm{C}$, washed 10 times in PBS and the plate developed by adding substrate solution consisting of one $20 \mathrm{mg}$ o-phenylenediamine tablet (Sigma) in $60 \mathrm{ml} 0.1 \mathrm{M}$ citrate buffer $\mathrm{pH} 5.5$ with $20 \mu$ of $30 \%$ hydrogen peroxide. The reaction was stopped with $20 \mu$ of $4 \mathrm{M}$ sulphuric acid after $20 \mathrm{~min}$ and the optical densities read in an MR5000 plate-reader (Dynatech). Antibody titres were calculated by subtracting the optical densities of the blank well from those of the ganglioside-coated well and plotting the resultant optical density versus the dilution on semilog paper. The dilution at which the baseline optical density (defined as 3 standard deviations above that obtained with second antibody alone) was crossed, was assigned as the antibody titre.

LM1. Gangliosides were separated on aluminium backed Kieselgel 60 $W_{254}$ S HP thin layer chromatography (TLC) plates (Merck Ltd, Poole, UK) in the solvent system chloroform:methanol:0.25\% potassium chloride in a volume ratio of 50:40:10. An aliquot of a standard ganglioside mixture containing LM1 (provided by Dr Marcus, Baylor College, Houston, Texas, USA). The chromatograms were air dried, dipped in $0.1 \%$ polyisobutylmethacrylate beads for $20 \mathrm{~s}$ at $40^{\circ} \mathrm{C}$, air dried, blocked with $1 \%$ BSA in PBS pH 7.5 for $1 \mathrm{~h}$, incubated with the test serum in appropriate dilutions in blocking buffer for $4 \mathrm{~h}$ at $4^{\circ} \mathrm{C}$, washed 5 times in blocking buffer, incubated for $2-3 \mathrm{~h}$ at $4^{\circ} \mathrm{C}$ with 
peroxidase-conjugated rabbit antihuman IgM diluted 1/3000 (Dako) and washed 5 times in blocking buffer. Plates were developed autoradiographically for 5-30 s using enhanced chemiluminescence procedure according to manufacturer's instructions (Amersham,UK).

\section{Immunoabsorption and purification for cold agglutinating antibody}

Cold agglutinating antibodies were purified from normal human red cells as follows: group $O$ red blood cells were washed 4 times in PBS $(0.01$ $\mathrm{M}, \mathrm{pH}$ 7.4). One volume of packed red cells was then incubated with 1 volume of plasma at $4^{\circ} \mathrm{C}$ with occasional mixing for $1 \mathrm{~h}$. This produced an aggregation of pellet that was centrifuged at $4^{\circ} \mathrm{C}$ ( 5 times, $2500 \mathrm{rpm}$ ) and washed 6 times in ice cold PBS with mechanical deagglutination at each wash. Following the last centrifugation, the wash supernatant was removed and replaced by one volume of PBS and the cells were gently mixed at $40^{\circ} \mathrm{C}$ for $5 \mathrm{~min}$. This mixture was centrifuged immediately (in buckets containing water at $40^{\circ} \mathrm{C}$ ) and the supernatant, which contained a fine milky precipitate, was transferred to a clean tube. This material is referred to as red blood cell purified IgM fraction (RBC-IgM). Immunoabsorption studies were also carried out by preincubation of an appropriate RBC-IgM solution with enzyme linked immunosorbent assay (ELISA) plates coated with GD3 and using the harvested supernatant for TLC overlay studies. Immunoglobulin isoelectric focusing and immunoblotting was based on standard techniques (Keir et al., 1990).

ELISA assays were also performed using $\mathrm{k}$ and $\lambda$ light chain specific antibodies.

As serum paraproteins were known to possess strong cold agglutinating activity, the binding properties to gangliosides were tested over a range of temperature including body temperature.

AntiGA1 and antisulphatide antibodies were considered to be high when the titres exceeded the laboratory upper limit of normal of 
1/5000. The normal upper limit of other antibodies titres (GM1, GM2, GM3, GD1a, GD1b, GD3 and globoside was 1/500. AntiMAG assay was performed by Western blot (Willison et al., 1993) and reported as positive or negative as the titre could not be quantified (Dr HJ Willison, personal communication).

\section{Histopathological investigation}

Sural (or, in some instances, radial) nerve biopsy was performed under local anaesthesia in 38 patients. Fascicular biopsy was usually performed, the specimen being taken from the sural nerve immediately posterior to the lateral malleolus or from the radial nerve at the wrist. The specimens were fixed for $3 \mathrm{~h}$ in $3 \%$ glutaraldehyde in PIPES buffer (Baur, Stacey, 1977) followed by post fixation in 1\% osmium tetroxide in the same buffer for $3 \mathrm{~h}$. Dehydration was in increasing concentrations of ethanol, followed by embedding in Araldite via 1.2 epoxypropane. For light microscopy, semithin $(1 \mu \mathrm{m})$ sections were stained with thionin and acridine orange (Sievers, 1971). Electron microscope sections $(60-80 \mu \mathrm{m})$ were contrasted with $12.5 \%$ uranyl acetate in methanol followed by $0.25 \%$ lead citrate. The sections were examined by $\mathrm{Dr} \mathrm{RH}$ $\mathrm{M}$ King in a JEOL $100 \mathrm{CX}$ electron microscope.

\section{Statistical methods}

Survival analysis was performed by constructing Kaplan-Meier survival curves, the differences being compared by a log rank test (Peto et al., 1977). Summed score results, when applicable, were compared by using nonparametric tests: Kruskal-Wallis analysis of variance for comparing independent groups and Wilcoxon signed ranks test for comparing paired data. Correlation of DML and maximal MCV was undertaken by linear regression and the difference between each group assessed by ANOVA. Differences were considered significant if $P<$ 
0.05. Only descriptive analyses were performed for groups of less than 5 patients. 


\section{Symptoms Score}

Score 0 point for absence of symptoms

1. Symptoms of muscle weakness

a. Bulbar

1. Extraocular

2. Facial

3. Tongue

4. Throat

b. Limbs

5. Shoulder girdle and upper arm

6. Hand

7. Glutei and thigh

$8 . \quad$ Legs

2. Sensory disturbances

a. Negative symptoms

9. Difficulties identifying objects in mouth

10. Difficulty identifying objects in hands

11. Unsteadiness in walking

b. Positive symptoms

12. "Numbness", "asleep feeling", "pricking" at any site

13. Pain: burning, deep aching, tenderness at any location

3. Autonomic symptoms

14. Postural fainting

15. Impotence in males

16. Loss of urinary control

17. Night diarrhoea

Total score for normal $=0$ 
SIGN SCORE:

Motor system Using MRC grading system

Upper limbs

Right

Left

deltoid

biceps

triceps

finger extensors

finger flexors

first DIO

Lower limbs

$$
\begin{aligned}
& \text { lliopsoas } \\
& \text { quadriceps } \\
& \text { hamstrings } \\
& \text { anterior tibial } \\
& \text { gastrocnemius } \\
& \text { peroneal } \\
\text { Normal score } & =\quad 120
\end{aligned}
$$

\section{Reflexes:}

biceps
triceps
brachioradialis
knee
ankle
2
1
0
$=\quad 20$




\section{Sensory System:}

\section{Touch \& pain}

$\begin{array}{ll}\text { normal } & 4 \\ \text { distal to wrist /ankle } & 3 \\ \text { distal to elbow/knee } & 2 \\ \text { distal to axilla/groin } & 0 \\ \text { normal score for touch }= & 16 \\ \text { normal score for pain }= & 16\end{array}$

Vibration (using $128 \mathrm{~Hz}$ tuning fork)

Middle finger/hallux 4

Ulnar styloid/medial malleolus 3

Elbow/knee 2

Clavicle/iliac crest $\quad 1$

No perception 0

Total normal score $=16$

Joint position sense

Middle finger/hallux, normal 2

Diminished 1

Absent 0

Total normal score $=8$

Total normal sensory score $\quad 56$

\section{SCORING FOR CLINICAL DISABILITY}

0 healthy

1 minor symptoms and signs of neuropathy capable of manual work

2 able to walk without support of a stick but incapable of manual work

3 able to walk with a stick, appliance or support

4 confined to bed or chairbound

5 requiring assisted ventilation

6 dead 


\section{PROFORMA I}

Name

d.o.b.

Hospital No.

\section{SYMPTOMS}

Onset

Description

SIGNS

Cranial nerves:

Motor system

Tremor $\quad \square$ yes $\quad \square$ no

Upper limbs

Lower limbs

Reflexes:

\section{SENSORY SYSTEM:}

Pain \& temperature Touch Vibration Joint position sense

Nerve thickening: $\quad \square$ yes $\quad \square$ no

Specify:

Others: 


\section{INVESTIGATIONS}

Paraprotein class and light chain:

Bone marrow:

Skeletal survey:

Bence-Jones proteinuria

Cerebrospinal fluid (CSF):

Others:

\section{NEUROPHYSIOLOGY}

\begin{tabular}{|l|l|l|l|l|}
\hline Date & & & & \\
\hline Motor study & & & & \\
\hline Upper limb & & & & \\
\hline MCV & & & & \\
\hline DML & & & & \\
\hline d-CMAP & & & & \\
\hline p-CMAP & & & & \\
\hline F latency & & & & \\
\hline Lower limb & & & & \\
\hline MCV & & & & \\
\hline DML & & & & \\
\hline d-CMAP & & & & \\
\hline p-CMAP & & & & \\
\hline F latency & & & & \\
\hline
\end{tabular}




\begin{tabular}{|l|l|l|l|l|}
\hline Sensory study & & & & \\
\hline Median & & & & \\
\hline Amplitude & & & & \\
\hline SNCV & & & & \\
\hline Ulnar & & & & \\
\hline Amplitude & & & & \\
\hline SNCV & & & & \\
\hline Radial & & & & \\
\hline Amplitude & & & & \\
\hline SNCV & & & & \\
\hline Sural & & & & \\
\hline Amplitude & & & & \\
\hline SNCV & & & & \\
\hline EMG & & & & \\
\hline
\end{tabular}

Other neurophysiological investigations: 
PROGRESS:

\begin{tabular}{|c|c|c|c|c|}
\hline Date & Clinical progress & M level & Other Ig & Treatment \\
\hline & & & & \\
\hline & & & & \\
\hline & & & & \\
\hline & & & & \\
\hline & & & & \\
\hline & & & & \\
\hline & & & & \\
\hline & & & & \\
\hline & & & & \\
\hline & & & & \\
\hline & & & & \\
\hline & & & & \\
\hline & & & & \\
\hline & & & & \\
\hline & & & & \\
\hline & & & & \\
\hline & & & & \\
\hline & & & & \\
\hline & & & & \\
\hline
\end{tabular}


PROFORMA II

Name

Diagnosis

\section{CURRENT SYMPTOMS}

Level of disability:

SIGNS:

Cranial nerves:

Motor system:

Tremor

$\square$ yes

$\square$ no

Upper limbs

Lower limbs

\section{Reflexes:}

Sensory system:

Pain \& temperature Touch Vibration Joint position sense

Nerve thickening: $\square$ yes $\square$ no

Specify:

Others: 
INVESTIGATIONS:

Paraprotein type and concentration:

Anti-MAG:

Antigangliosides:

Others:

Histopathology:

Others:

Neurophysiology

Sensory study

Motor study

Outcome:

Years of follow-up:

Malignancy: $\quad \square$ yes $\quad \square$ no

Specify: 


\section{Questionnaire to physician or general practitioner}

Patient's name:

Address:

d.o.b.:

Hospital no:

Diagnosis:

From:

Address:

1. Which of these scales best describes your patient's disability at the present?

a. Minor symptoms and signs of neuropathy capable of doing manual work.

b. Able to walk without support of a stick but incapable of manual work.

c. Able to walk with a stick, appliance or support.

d. Confined to bed or chairbound.

e. Requires assisted ventilation.

f. Dead.

2. If the answer is $f$ ) in 1

a. What was the cause of death?

b. When? At what age?

c. Have any other disease(s) been diagnosed after the diagnosis of polyneuropathy was made? (including malignancy of haematological and nonhaematological nature).

3. What do you think is (was) the course of the patient's neurological condition?
a. Static.
b. Slowly and gradually progressive.
c. Rapidly progressive.
d. Stepwise deterioration. 
4. If the patient is still alive, when did you last seen the patient?
a. The last 3-6 months.
b. 6-12 months.
c. 1-2 years.
d. 2-5 years.
e. $>5$ years.

5. The pattern of peripheral neuropathy is

(There will be more than one answer to this question)
a. Sensory only.
b. Sensorimotor
c. Distal.
d. Proximal.
e. Symmetrical.
f. Patchy and asymmetrical.

6. Any cranial nerve involvement?
a. No.
b. Yes.

Please specify:

7. Any autonomic involvement?
a. No
b. Yes

Please specify:

8. In your opinion, has the treatment made the difference to the neurological condition?
a. No.
b. Yes.

9. If the answer is yes in 8

Please specify what treatment: 
Was the improvement sustained and for how long?

10. Have any other diseases developed including malignancy?

a. No

b. Yes

Please specify which system:

When:

Any other comments: 
Questionnaire to patient

Name:

Address:

DOB:

Telephone no.:

Please tick the answer or answers that best describe your present symptoms:

1. Do you have the following?
a. tingling
b. pins \& needles
c. numbness
d. burning sensations

2. Please specify the extent and location
a. in the legs, mainly feet
b. in the legs, feet up to knees
c. in the legs, up to thighs
d. in the arms, hands only
e. in the arms, hands up to forearms
f. involving the whole of the arms
g. fairly symmetrical involvement on both sides
h. definitely one side more than the other

3. Do you have any weakness?
a. lifting your arms above the shoulders
b. affecting your hand grip
c. getting up from a low chair
d. foot drop or tendency to trip when walking

4. Do you notice any thinning of the muscles?
a. yes
b. no
If yes, please specify where 
5. Do you have
a. double vision
b. slurred speech
c. difficulty in swallowing

6. Do you have tremor?
a. yes
b. no

7. If the answer is yes,
a. where is it noticed? (e.g. just in the arms?)
b. is the tremor much the same
c. worse than before
d. better than before, as a result of treatment
e. better by itself

8. Do you think that on the whole, you
a. remain much the same
b. better
c. slightly worse
d. much worse than before

9. If the answer in question is $c$ ) or d) in 8 The progress has been
a. steadily and gradually worse
b. in a stepwise fashion? any precipitating factors?

10. Which category fits you best at the present
a. capable of doing manual work
b. able to walk without support of a stick but incapable of manual work
c. able to walk with a stick, appliance or support
d. confined to bed or chair bound

11. Did you have or are you on any treatment for your neuropathy?
a. no
b. yes
please name the medication(s): 
12. On balance, do you think the treatment you received has made any different to your condition?
a. no
b. yes

13. Any other comments:

If there is any difficulty in completing the form, please contact Dr S

Ponsford, Monday-Friday at 01203-538775. 


\section{CHAPTER 3}

\section{RESULTS}

\subsection{Patients}

Demographic data

Deceased patients

Surviving patients

Immunological subclassification

Clinical features (including the deceased patients)

Results of summed scores

\subsection{Neurophysiological findings}

Sensory study

Motor study

Motor conduction velocity

Distal motor latency

Distal compound muscle action potentials

Residual latency

Terminal latency index

Other neurophysiological findings

\subsection{Other laboratory findings}

\subsection{Neuropathological findings}

\subsection{Illustrative cases}




\section{RESULTS}

\subsection{Patients}

\section{Demographic data}

Fifty-five patients were studied, 41 were male. The duration of followup was 5-41 y with a mean of $14.2 \mathrm{y}$. Thirty eight patients had an IgM paraprotein ( $\kappa 23, \lambda 13$, unclassified 2$), 11$ an $\lg \mathrm{g}(\kappa 6, \lambda 5)$ and 1 an $\lg A \lambda$. Five had CIDP. The mean duration of the disease from the onset of neurological symptoms was 13.9 y (5-35) for the $\lg M, 13.3 \mathrm{y}$ $(5-26)$ for the $\operatorname{lgG}$, and 17.6 y $(7-41)$ for CIDP. The duration of the illness was $18 \mathrm{y}$ for the $\operatorname{lgA}$. Mean age at the onset of neuropathic symptoms was $56.9,57.4,52,36.5$ y for $\operatorname{lgM}$, IgG, IgA, and CIDP respectively. As the number of $\lg G, \lg A$ cases is small, $\lg G$ and $\lg A$ cases will be combined as a single group. Male:female ratio was 29:9, 7:5, 5:0 in patients with IgM, IgG/A paraproteins and CIDP.

\section{Deceased patients}

Fifteen patients died: 13 had IgM, 2 had IgG gammopathy. Only 3 out of 15 developed haematological malignancy. One (case no. 34) developed Waldenström macroglobulinaemia from a B cell lymphoma at the age of $65,23 \mathrm{y}$ after the onset of symptoms related to an $\operatorname{lgM}$ demyelinating neuropathy. The diagnosis was prompted by a sudden rise in the level of the paraprotein associated with malaise. He died from aspiration pneumonia following emergency total colectomy for ulcerative colitis at the age of 67. Case no. 49 developed refractory anaemia with excess blasts at the age of $73,11 \mathrm{y}$ after the onset of symptoms of neuropathy associated with an $\lg \mathrm{\lambda}$ paraprotein. The last patient (case no. 33), who originally had a benign $\lg M \lambda$ paraproteinaemic neuropathy developed acute myeloid leukaemia 17 y after the onset of his neurological symptoms. All 3 patients had been 
on immunosuppressive therapy for at least $2 \mathrm{y}$. Four out of 15 developed other malignancies: carcinoma of the coion, pancreas, pharynx and liver metastasis from an unknown primary. One patient with an IgM $\kappa$ paraproteinaemic neuropathy (case no 16) has recently been diagnosed and treated for carcinoma of the stomach. The other (case no 9) had recto-colectomy for carcinoma of the rectum and remained asymptomatic for gastrointestinal symptoms for the last $4 \mathrm{y}$. Both had never received immunosuppressive drugs. The remaining patients died from unrelated nonmalignant illnesses. Details of the patients, their outcomes and cause of death are summarised in Tables 3.1 and 3.2 .

Kaplan-Meier log rank tests failed to show any significant difference in the survival at a $90 \%$ confidence level for the following variables: sex; serum concentration, type, light chain, classification or antigenic properties of the paraprotein; presence or absence of immune paresis; degree of disability; type of neurophysiological abnormality. Some of the results are given in Fig. 3.1.

\section{Surviving patients}

\section{Immunological subclassification}

Data for the remaining 39 patients are summarised in Tables 3.3, 3.4, 3.5. Patient no. 38 with an IgM paraprotein who also had a chromosome 17p11.2 duplication and a generalised demyelinating neuropathy (Gregory et al., 1993) was excluded from the analysis but his case history is given at the end of this chapter. Patient 1 in the IgG/A group (case no. 39) could not be adequately assessed for symptoms and signs as he had severe cognitive impairment related to hypertension and small vessel cerebrovascular disease.

Based on the neuroimmunological results, the 24 patients with an IgM paraprotein can be subdivided into 5 groups: 6 with high titres of IgM antisulphatide activity (anti-S), 7 with antiMAG activity, 3 with combined antiMAG and anti-S activities, 2 with $\operatorname{lgM}$ activity against 
disialosyl group and 6 with unidentified antibody activity. Patients with IgG/A and CIDP did not have demonstrable antibody activity against the aforementioned antigens.

The mean age of onset was 63.3 y (48-75) in the anti-S, 56.7y (48-72) in antiMAG, 53.6y $(49-60)$ in antiMAG and anti-S, 34y $(28,40)$ in antidisialosyl and 52.7y (45-58) in those with unidentified antibody activity. The mean duration of follow-up from the onset of the neurological symptoms was 12.3 (6-16), 13.4 (6-22), 13.3 (9-21), 17 $(7,26)$ and $10.8(5-18)$ y respectively.

Clinical features (including deceased patients)

All patients presented with sensory symptoms consisting of unsteadiness (from sensory ataxia) and/or numbness and/or paraesthesiae. These began either in the legs or hands and legs simultaneously and progressed proximally. Two cases with IgG paraproteins (18.2\%) and 2 out of 5 patients (40\%) with CIDP presented with sensory disturbances confined to the hands. Five patients with IgM paraproteins (13.5\%), 4 patients (36.4\%) with IgG/A paraproteins and 4 cases $(80 \%)$ with CIDP had paraesthesiae affecting the face. Two of the former had high titres of IgM anti-S activity whilst the other 2 had $\operatorname{lgM}$ activity against a disialosyl group. The type of antibody activity in the remaining patient with facial sensory symptoms could not be determined as he had died. Fourteen patients with IgM $(38 \%), 4$ with IgG $(36 \%)$, the single patient with an IgA paraprotein and $1(20 \%)$ with CIDP had prominent dysaesthesia. This recovered in 4 of the $\lg M$ and 2 of the $\lg G$ cases and in the $\lg A$ case, after 3-15 $y$.

Just over $50 \%$ of patients complained of weakness but on examination all patients had at least mild but definite motor involvement. This began or was worse in the lower limbs apart from 3 patients with IgG paraproteins whose weakness was more pronounced in the arms. Two of these 3 patients had bilateral facial weakness and 1 had facial sensory symptoms, which were responsive to corticosteroid 
or intravenous immunoglobulin treatment. Two patients (nos. 17,18) with IgM antidisialosyl activity had intermittent diplopia. Patient 18 developed a subacute episode of bilateral facial weakness, ophthalmoplegia, bulbar palsy, global weakness and respiratory insufficiency necessitating assisted ventilation after elective hip replacement at the age of $52 \mathrm{y}$. He recovered to his preoperative state with intravenous immunoglobulin therapy. He had received no treatment before or after this acute episode and deteriorated slowly from walking with sticks to wheelchair-bound over $26 \mathrm{y}$. Case 17 also experienced considerable improvement after intravenous immunoglobulin therapy from walking with a stick to being able to walk unaided. The effect only lasted for 4-5 wk. The weakness in 1 of the patients with CIDP was confined to the left facial muscles.

Tremor was the presenting feature only in patients with IgM paraproteinaemia (50\%) but at the final follow-up examination, 32 out of $36(88 \%)$ with $\mathrm{IgM}$ and 9 out of $11(82 \%)$ with $\mathrm{IgG} / \mathrm{A}$ paraproteins, and 2 (40\%) with CIDP had tremor, in some of whom it was incapacitating. Pseudoathetosis was observed only in patients with IgM paraproteinaemia ( 8 out of $37,21.6 \%$ ): 1 had a high IgM anti-S titre, 3 had antiMAG activity, 1 had antidisialosyl activity and 2 had unidentified antibody activity. The other patient died. Four of these patients had very slow maximal MCV: $2.7,5,9,11 \mathrm{~m} / \mathrm{s}$; the first 3 values were the lowest in the whole series. For the remaining patients, MCV was between 20 and $25 \mathrm{~m} / \mathrm{s}$. All had prominent large fibre deficits on clinical examination.

Eight patients (21.6\%) with $\mathrm{IgM}$ and $2 \mathrm{IgG} / \mathrm{A}$ (18.2\%) paraproteins were found to have peripheral nerve thickening other than at the common entrapment sites.

The polyneuropathy had a slowly progressive course with the exception of 3 cases in the $\operatorname{lgM}(8.1 \%), 7$ in the $\operatorname{lgG} / \mathrm{A}(58 \%)$ group and 3 in the CIDP (60\%) groups, all of whom had a relapsing remitting course. Two of the IgM cases had antidisialosyl antibodies; the third 
died. Of the remaining patients, a relapsing/remitting course developed spontaneously in 3 patients ( 2 antidisialosyl, $1 \lg A$ ). For IgG case 45 , relapse occurred with a viral infection. The milder relapses took 6-8 wk to recover without treatment. The rest took place either on withdrawal or reduction of steroid therapy.

\section{Results of summed scores}

Comparison of summed scores of symptoms, signs and disability between the first and last visit are summarised in Figs 3.2 and 3.3. This could only be performed in patients who were still alive.

There was a significant difference in the summed scores between the patients with IgM and IgG/A (Figs. 3.2.1-3.2.8) in motor deficits, tendon reflexes, all sensory tests and disability when the patients were first seen. Similar differences were observed at the final examination apart from the motor deficits. Significant change in the summed scores of the same group of patients between the first and last visits was seen only in the $\lg M$ group for tendon reflexes $(P=0.001)$, joint position sense $(P=0.001)$ and disability $(P=0.001)$. Improvement in motor, tendon reflex and disability scores was observed only in the $\lg$ /A group and was seen in 6 of $8(75 \%)$ of $\lg$ cases. This was associated with steroid therapy in 5 cases. There was no significant difference between the 3 IgM subgroups: anti-S, antiMAG and unidentified antibodies, at the time of the initial and final examinations (Figs 3.3.1-3.3.8). More parameters were observed to have significant deterioration between the 2 visits in the antiMAG group.

\subsection{Neurophysiological findings}

The mean time intervals between the initial and the last nerve conduction studies were $8.9 \mathrm{y}$ for the $\operatorname{IgM}(9,10,8.7,9.5,7.6 \mathrm{y}$ for anti-S, antiMAG, antiMAG+S, antidisialosyl and unidentified antibody subgroups, respectively), $10.4 \mathrm{y}$ for $\lg \mathrm{G} / \mathrm{A}$ and $11.8 \mathrm{y}$ for CIDP. 


\section{Sensory study}

Only the presence or absence of the SAPs was taken into account due to numerous anticipated variabilities. Data on the frequency of the reversion of SAPs either from absent to present or vice versa are summarised in Table 3.6.

When all patients were first seen, the SAPs were either uniformly or patchily abnormal and more severely so in the lower limbs. Retention of SAPs or an improvement on follow-up study was observed only in the patients who had IgM with unidentified antibody activity and IgG/A paraproteins and CIDP. Improvement was considered when there was a reversion from absent to present SAPs or an increase of SAP amplitude of more than $50 \%$.

The pattern of abnormal median and normal sural sensory response (Bromberg, Albers, 1993) was seen in 4 patients: 1 with an unclassified IgM paraprotein (deceased case 25), 3 with $\lg$ paraproteins (case 39, 44 and 45). The "extreme pattern" of absent median and normal sural sensory response was seen in cases 44 and 45. Sural SAPs were absent in the first $2 \lg$ cases after 5 y whilst the pattern was retained during the final follow-up in case $45,8 y$ from the onset of his symptoms.

\section{Motor study}

\section{Motor conduction velocity (MCV)}

Changes in MCV are summarised in Table 3.7. An improvement or deterioration was considered when there was an increase or decrease in the value of MCV $\geq 8 \mathrm{~m} / \mathrm{s}$ (Oh, 1993a).

Similar changes were seen in MCV. Lower limbs were more affected. Nerves with unrecordable potentials were commonly found in the legs and in all patients with IgM antiMAG activity. Improvement was only seen in patients other than those with IgM paraproteinaemia. 
Upper limb MCVs on the last follow-up are listed along with other parameters in Table 3.8.1. MCVs in the legs were not infrequently unobtainable either because of completely denervated foot muscles or inexcitable nerves.

Distal motor latency (DML)

Distal compound muscle action potentials (distal CMAP)

Residual latency $(R L)$

Terminal latency index (TLI)

Mean DML and DCMAP are listed in Table 3.8.1 with a scattergram for the DML of patients with demyelinating neuropathy in different groups in Fig 3.4.

There was no significant difference at the $10 \%$ confidence level between DML of various groups or subgroups of patients with IgM paraproteinaemia. The highest DML value belonged to a patient with IgG gammopathy (case no.42). There was a significant prolongation of DML in comparison with the MCV in the forearm for the IgM cases both with anti-S and antiMAG activity when the patients were first seen. This correlation was no longer evident at the final follow-up (Table 3.9). There was little correlation between the terminal latency index and the type of paraprotein for the median nerve at the follow-up examination (Fig. 3.5) although a tendency for a relative distal prolongation was still evident in the ulnar nerve for the antiMAG and anti-S patients (Fig. 3.4).

The mean dCMAP was significantly low in amplitude in the IgM cases with antiMAG activity in comparison with other groups $(P<0.05)$. No statistically significant difference could be demonstrated in the other parameters.

All patients had a sensorimotor neuropathy with a degree of axonal degeneration at their last follow-up. Two patients (cases 1 and 22) had an axonal neuropathy whilst the rest had a predominantly demyelinating neuropathy. Patients with antiMAG IgM activity had a significantly prolonged ulnar residual latency in comparison with the 
other IgM subgroups, the IgG/A and CIDP. None had conduction block. Six of the patients with unidentified IgM antibody activity, 4 in the igG/A group and 4 out of 5 of CIDP cases had asymmetric demyelination with evidence of conduction block.

\section{Other neurophysiological findings}

Only 4 patients (cases $13,28,42,43$ ) had magnetic stimulation for evaluation of central motor conduction time and motor conduction in the proximal segment. Cases 13 and 28 with IgM paraproteinaemia, the former with antiMAG activity, had fairly uniform slowing of ulnar motor conduction velocity from the spinal cord to the wrist. The other 2 with IgG paraproteinaemia showed an asymmetric reduction of $\mathrm{MCV}$ in various segments (Table 3.10). Central motor conduction time performed in 2 cases $(13,43)$ was normal $(7,9 \mathrm{~ms}$ respectively).

Evoked potentials responses were carried out in 5 cases: pattern reversal visual evoked potentials in cases 10,15,18, 30 and 34. These were normal in cases 10,18 and 30 . Case 15 with IgM antiMAG + S activity and case 34 with unclassified IgM activity (the patient had died) had fairly symmetrically delayed $\mathrm{P} 100$ values (right $=118$, left $=122$ $\mathrm{ms}$; right $=129$, left $=130 \mathrm{~ms}$ respectively), the normal upper limits in the laboratory being $112 \mathrm{~ms}$. Case 15 had bilateral optic atrophy. Case 34 had proptosis but normal fundoscopy. Both had normal visual acuity. Cases 2, 15 and 34 had short latency brainstem evoked potential recordings that were normal. A median somatosensorv evoked potential recording was carried out in case 34. Erb's and cervical potentials were absent whilst there was a marked delay in cortical potentials bilaterally: right median nerve stimulațion $=47 \mathrm{~ms}$, left median nerve stimulation $=48 \mathrm{~ms}$.

\subsection{Other laboratory findings}

The CSF protein concentration was elevated in 18 out of $21 \mathrm{IgM}$ cases (0.6-3.0 g/l, mean 1.06) in which this was examined, in 7 out of 7 of the 
IgG/A cases (0.7-4.8 g/l, mean 1.77) and 3 out of 5 with CIDP (0.6-2.46 g/l, mean 1.24).

The paraprotein was detected in the blood only on repeating the test approximately $1 \mathrm{y}$ after the first examination in 3 cases but immunofixation had not been carried out on the first occasion. Four patients (IgM cases 16,18, 24 and IgG case 41) initially with monoclonal paraproteins had a bi or triclonal gammopathy at their last follow-up. Of the $3 \mathrm{lgM}$ cases, the biclonal bands were $2 \mathrm{lgM \kappa}$ bands in case 16 . Cases 18 and 24 had triclonal bands consisting of $1 \operatorname{lgMK}$ and $2 \lg M \lambda$ and $2 \lg M \lambda$ and $1 \lg A \lambda$. Case 41 initially with an $\lg G_{\kappa}$ band had $\lg G_{\kappa}$ and $\lg M \lambda$ bands. No paraprotein band was detected at the last followup in 2 patients, one originally with an IgG (case 45) and the only case with an IgA paraprotein (case 50), the latter with clinical and electrical improvement. There was no immune paresis in any of the patients.

Screening for autoantibody activity was performed in nearly all the patients but was positive in 1 patient with IgM antiMAG $+S$ activity (case 14) having antimitochondrial antibody $>1 / 15,000$. Case 15 was found to have positive antineutrophil cytoplasmic antibody (ANCA).

\subsection{Neuropathological findings}

These are summarised in Table 3.11. The determination of nerve fibre pathology was based on the appearances in semithin sections and electron microscopy and from teased fibre preparations whilst the deposition of $\mathrm{lg}$ and complement in nerve was assessed by the direct immunoperoxidase method on tissue sections. For the latter, C3d and C1q were not tested. Nerve biopsies were usually performed when the patients were first assessed.

In the specimens with demyelinating pathology, hypertrophic changes (onion bulbs) were not infrequently observed. Even though inflammatory cells were reported, they were sparse and mainly found around the blood vessels in the epineurium and endoneurium. 
Unfortunately data on the presence of widely spaced myelin were incomplete. Of the few available results, these were present only in patients with IgM paraproteinaemia. Likewise immunoglobulin deposition was only seen in the IgM paraproteinaemic group. IgM deposition was not seen in the endoneurium but was always present in the perineurium both in the normal and abnormal nerves.

The pathological findings in some of the patients are shown in Figs 3.6.1-3.6.8.

\subsection{Illustrative cases}

Case 9

This 72 y old Irish ex-labourer presented with a progressive history of difficulty in walking and unsteadiness starting at the age of $61 \mathrm{y}$. When he was admitted for investigation $1 \mathrm{y}$ after the onset of his symptoms, he was found to have a high steppage gait, mild ataxia and mild intention tremor in the upper limbs. There was bilateral sensorineural deafness, mild weakness of the arms and weakness and wasting of the legs distally. Tendon reflexes were absent. Touch and pain sensation was impaired over the toes. Vibration sense was absent to the costal margin bilaterally whilst joint position sense was impaired at the big toes. Peripheral nerves were not thickened. Subsequently his symptoms slowly deteriorated but he was able to remain in full and then part time employment for the next $4 \mathrm{y}$. Prior to his retirement, he developed transient proximal weakness predominantly in the legs following rectocolectomy for carcinoma of the rectum. When he was last seen $11 \mathrm{y}$ after the onset of his symptoms, he walked with one stick and footdrop splints and was dependent on his wife. He had no symptoms suggestive of cranial nerve or autonomic dysfunction. The patient had never received any treatment for the neuropathy.

Examination revealed marked symmetrical wasting of the intrinsic hand muscles and both legs below the knees. Both extensor digitorum brevis muscles were absent. There was postural and intention 
tremor of both hands. He had mild weakness of forearm muscles and marked weakness of the small hand muscles and all the muscles below the knees. The tendon reflexes were absent. Pain and temperature sensation was absent at both wrists and below the knees. Touch sensation was absent in the distal one third of the legs. Vibration was absent to the elbows and iliac crests. Joint position sense was lost at the fingers and big toes. The peroneal and ulnar nerves were thickened. General physical examination was normal.

Routine haematology including erythrocyte sedimentation rates, biochemistry, serum vitamin B12, folate, serum calcium, liver function tests, mid stream urine, urinary Bence-Jones protein, chest $x$-ray, skeletal survey, and bone marrow examination were normal or negative. Cerebrospinal fluid protein was $0.7 \mathrm{~g} / \mathrm{l}$ with no pleocytosis. Serum protein electrophoresis and immunoelectrophoresis showed an IgMK, $8.2 \mathrm{~g} / \mathrm{l}$ at the initial examination. The level varied between $3.6-$ 5.7 and was at $6.8 \mathrm{~g} / \mathrm{l}$ at the last follow-up. IgM antibody to MAG by Western blot analysis was positive but other antiglycolipid antibodies were negative.

Nerve conduction studies performed on his initial admission showed all SAPs (right median, ulnar, radial, sural) to be absent. There was no response on stimulating the peroneal nerve at the ankle. Right median nerve $D M L=15.2 \mathrm{~ms} ; \mathrm{MCV}=15 \mathrm{~m} / \mathrm{s} ; \mathrm{CMAP}=0.5 / 0.5 \mathrm{mV}$ at the wrist and elbow; right uinar nerve $D M L=10.4 \mathrm{~ms} ; M C V=13 / 17 \mathrm{~m} / \mathrm{s}$ at the forearm, elbow segment; CMAP $=2 / 2 / 2 \mathrm{mV}$ at wrist, below elbow and above elbow respectively. Left ulnar nerve $\mathrm{DML}=10.1 \mathrm{~ms} ; \mathrm{MCV}$ $16 / 22 / 18 \mathrm{~m} / \mathrm{s}$ at the forearm, across the elbow and elbow to axilla segment; CMAP = 3/2/2/2 $\mathrm{mV}$ at the wrist, below, above the elbow and axilla. SAPs and peroneal nerve response remained absent at the time of final follow-up. Right ulnar nerve DML $=13 \mathrm{~ms} ; M C V=12 / 13 / 15 \mathrm{~m} / \mathrm{s}$ with CMAP of 2/2/2/2 at wrist, below elbow, above elbow and axilla. Minimal F wave latency was $56 \mathrm{~ms}$. Residual latency was 8.1 and TLI of 0.4. Serial electromyography $(1988,1990,1992,1997)$ showed 
progressive denervation which became more severe in the distal muscles, spreading more proximally.

Light and electron microscopic examination of the right sural nerve biopsy specimen showed severe loss of myelinated fibres. The remaining fibres were small and thinly myelinated indicating remyelination or axonal regeneration (Fig 3.6.1b, 3.6.2b). Occasional onion bulbs and tomacula were seen. At least $50 \%$ of the fibres showed widely spaced myelin, most frequently affecting the outermost lamellae. The presence of stacks of flattened Schwann cell process and 'collagen pockets' suggested loss of unmyelinated axons (Fig. 3.6.8). There was marked endoneurial oedema but the perineurium and the connective tissues including blood vessels were normal. No inflammatory cells were seen. Immunohistochemistry showed positive staining only for IgM on the surviving myelin sheaths, subperineurium and within the perineurium. The detailed neuropathological changes have been reported by Jacobs and Scadding (1990).

Comment: Case 9 typifies the patients with benign IgM paraproteinaemic neuropathy with antiMAG activity. The patients are usually male and present in their late fifties with predominantly large fibre (particularly vibration sense) neuropathy worse in the legs. Postural or intention tremor develops early in the upper limbs. There is no craniobulbar or autonomic involvement. Symptoms progress very slowly and gradually to produce significant motor and sensory deficits. The IgM usually has a $\mathrm{k}$ light chain. Nerve conduction studies show a predominantly demyelinating neuropathy. SAPs are usually absent and motor responses in the lower limbs are absent. Distal motor latencies are markedly prolonged with slow MCV. The latter is fairly symmetrical in the more proximal segments either in the same or different nerves of the same limb. Progressive axonal degeneration (which is best demonstrated by serial electromyographic examination) occurs despite a relatively stable MCV and DML. Paraprotein levels are usually low 
( $<10 \mathrm{~g} / 1)$ and nerve biopsies typically show widely spaced myelin on surviving demyelinated/remyelinated fibres.

\section{Case 18}

This $54 \mathrm{y}$ old retired gunsmith was fit and well until at the age of $28 \mathrm{y}$ when he had an episode of vertigo, diplopia and paraesthesiae in the hands. These symptoms cleared except for 'pins and needles' in the hands which persisted. At the age of 32 , he noticed slight numbness of his feet and more extensive sensory disturbance in the upper limbs. He started to experience deterioration with his balance at the age of 35 when he began to trip. He also had difficulty with manual dexterity. Just prior to his first assessment at the age of 38 , he noticed wasting and weakness in both hands. There was no relevant family history and no precipitating factors were evident.

On examination the cranial nerves were normal apart from the pupils that were regular, unreactive to light but responding briskly on convergence. There was marked wasting of the small hand and extensor muscles of the forearms, early wasting of both extensor digitorum brevis muscles and clawed toes. Pseudoathetosis of his outstretched hands was observed. He had mild proximal and more severe distal weakness in the upper limbs and mild to moderate weakness in the legs. There was slight finger/nose and heel/shin ataxia. The tendon reflexes were all absent and the plantar responses were flexor. Pain and temperature sensation was reduced to elbow and mid shin levels. There was loss of touch sensation on the soles of the feet. Vibration sense was absent to the wrists and iliac crests. Joint position sense was absent in the toes and reduced in the fingers. Both peroneal nerves were thickened. He had a bilateral foot drop gait and mild ataxia and showed Rombergism. He was discharged home without treatment. Since then he has progressively deteriorated especially in his balance and for fine finger movements. At the age of 52 , he developed a subacute episode of bilateral facial weakness, 
ophthalmoplegia, bulbar palsy, moderate global limb and trunk weakness and respiratory insufficiency necessitating assisted ventilation. This occurred after an elective hip replacement for fracture of the right femur following a fall. He recovered to his preoperative state with intravenous immunoglobulin therapy. When he was last examined, he could walk very short distances with a frame and could feed himself slowly. His balance and hand movement continued to deteriorate. On examination he had pseudo Argyll Robertson pupils. There was postural upper limb tremor and early clawing of the fingers in addition to the previously noted wasting distal to the elbows and knees. $\mathrm{He}$ had a mild proximal weakness and severe distal weakness in the upper limbs and a severe weakness of the muscles of the legs distally. All tendon reflexes were absent. Pain, temperature and touch sensation was absent below mid palms and ankles. Vibration sense was only detected at the clavicles and was just perceptible at the iliac crests. Joint position sense was absent on the fingers and at/distal to ankles. Peripheral nerves were diffusely thickened.

Routine haematology including erythrocyte sedimentation rate, biochemistry, MSU, serum calcium, liver function tests, blood and CSF syphilitic serology, T4, phytanic acid, serum B12 and folate, bone marrow examination, chest $\mathrm{x}$-ray and skeletal survey were normal. CSF was acellular with protein of $0.62 \mathrm{~g} / 1$. An $\lg M \lambda$ paraprotein at $5 \mathrm{~g} / \mathrm{l}$ was found with normal IgG/A levels at the initial assessment. Three monoclonal bands were seen on the last follow-up: $1 \lg M \kappa$ and $2 \lg M \lambda$ with a total concentration of $8.9 \mathrm{~g} / \mathrm{l}$ and no immunoparesis. There was no urinary Bence-Jones protein. IgM activity against MAG was negative but it was strongly positive against GD1a, GD1b, GT1b, GQ1b, GD3, GM3 and sulphatides (>12500, normal upper limits < 500 except for sulphatides $>5000$ ). Cold agglutinin antibodies were also positive.

Initial nerve conduction studies showed absent sensory potentials. For the right median: $D M L=10.2 \mathrm{~ms}, \mathrm{MCV}=27 \mathrm{~m} / \mathrm{s}, \mathrm{CMAP}$ 
$=2.1 / 1.1 \mathrm{mV}$; right ulnar: $\mathrm{DML}=5 \mathrm{~ms}, \mathrm{MCV}=27 \mathrm{~m} / \mathrm{s}, \mathrm{CMAP}=1.4 / 0.3$ $\mathrm{mV}$; right peroneal: $\mathrm{DML}=10.8 \mathrm{~ms}, \mathrm{MCV}=30 \mathrm{~m} / \mathrm{s}, \mathrm{CMAP}=0.7 / 0.3$ $\mathrm{mV}$. When these were repeated at the last follow-up 25 y after the initial onset of diplopia, all SAPs were absent. Rt median: $D M L=8.8$, MCV $=27 / 39 \mathrm{~m} / \mathrm{s}, \mathrm{CMAP}=4 / 2.5 / 1 \mathrm{mV}$ (no dispersion) at the wrist , elbow and axilla; minimal $F$ wave latency was $77-83 \mathrm{~ms}$; right ulnar: $\mathrm{DML}=7 \mathrm{~ms} ; \mathrm{MCV}=20 \mathrm{~m} / \mathrm{s}, \mathrm{CMAP}=0.2 / 0.1 \mathrm{mV}$ at the wrist and above elbow; right median $\mathrm{RL}=6.58 ; \mathrm{TLI}=0.252$; right ulnar $\mathrm{RL}=4.25 ; \mathrm{TLI}=$ 0.39. A nerve conduction study could not be reliably performed in the lower limbs due to marked pitting oedema. Pattern reversal visual evoked potentials performed during his first assessment were within normal limits $(114,116 \mathrm{~ms}$ on the right and left respectively, the normal upper limit being $118 \mathrm{~ms}$ ). Autonomic function tests were also normal.

Right radial nerve biopsy showed severe demyelination with remyelination and occasional small onion bulbs. $A$ number of regenerative clusters were seen even though there was no active axonal degeneration. Connective tissues were normal and widely spaced myelin was not noted. Immunofluorescent studies were negative for Ig deposition and C3.

Comment: Although case 18 had a benign IgM neuropathy with predominantly large fibre sensory involvement, there were several unusual features. First the age of onset was significantly early. Secondly there were acute/subacute episodes of craniobulbar nerve involvement which occurred on a background of a slowly progressive ataxic neuropathy. The pseudo Argyll Robertson pupils were thought to be due to parasympathetic dysfunction (Williams, Harding, 1985). The initial episode occurred without any predisposing event and resolved spontaneously whilst the most recent and more severe episode resembling the Miller-Fisher syndrome recovered with intravenous immunoglobulin therapy. The pattern of weakness involved proximal as well as distal muscles and was more pronounced in the upper limbs. Nerve conduction studies showed conduction block (by the amplitude 
criterion) at the elbow. The markedly prolonged right median DML in comparison to the right ulnar DML could not be taken into account because of the high incidence of an additional entrapment lesion at the former site. The salient features are the immunological findings of $\operatorname{lgM}$ activity against the disialosyl group of gangliosides and $P$ antigen on red cell membrane. The patient thus conformed to the increasingly recognised group of patients which was given the acronym of CANOMAD by $\mathrm{Dr} \mathrm{H} J$ Willison (chronic ataxic neuropathy, ophthalmoplegia, monoclonal $M$ protein, cold agglutinins and disialosyl antibodies) (Paparounas et al., 1999).

\section{Case 38}

This patient, a botanist, was excluded from the case analyses because he was shown also to have type 1a hereditary motor and sensory neuropathy (HMSNla) with a chromosome 17p11.2 duplication (Gregory et al., 1993). He is now aged $54 \mathrm{y}$. He was orphaned at the age of $2 y$ and thus his early history is not known. He was noted to have 'bandy legs' as a child, attributed to rickets. He never ran well as a child but he played cricket to a good standard until the age of $25 \mathrm{y}$. He has one healthy brother.

The patient began to trip at the age of 38 . After 6 mo he developed numbness and paraesthesiae in his hands with decreased manual dexterity. At the age of 39 , numbness and paraesthesiae spread to his legs. His gait became progressively more unsteady. His sensory symptoms deteriorated with throbbing and burning paraesthesiae in both hands. By the age of 42 , despite lower limb orthoses he was only able to walk with a frame. He was investigated at a neurological centre at this stage when a diagnosis of chronic inflammatory demyelinating polyneuropathy was questioned. $\mathrm{He}$ received a course of plasma exchange with transient improvement but there was no response to 2 further courses. He then received 2 
courses of prednisolone at a dose of $60 \mathrm{mg} / \mathrm{d}$ for 3 wk with subjective but no objective deterioration.

Investigations at another centre at the age of 44 revealed normal results for routine haematology and biochemistry. Serum protein electrophoresis was found to be normal on repeated testing. Electrodiagnostic studies showed inexcitable nerves distally in all 4 limbs. MCV was $10 \mathrm{~m} / \mathrm{s}$ in the ulnar nerve on recording from flexor carpi ulnaris. SAPs were all absent. A sural nerve biopsy demonstrated a demyelinating neuropathy with prominent hypertrophic changes. No inflammatory infiltrates were seen

He was first seen at the National Hospital for Neurology and Neurosurgery, Queen Square, at the age of 47 when examination revealed normal cranial nerve function. There was generalised wasting and weakness both in the upper and lower limbs with pseudoathetosis of the fingers with the arms outstretched. He showed an ataxic steppage gait and walked using a frame. Romberg's test was positive. The tendon reflexes were all absent and plantar responses were not obtainable. He showed impairment of all sensory modalities distally in his limbs and reduced cutaneous sensibility over the anterior abdominal wall. Kyphoscoliosis was noted but there was no foot deformity. His peripheral nerves were enlarged.

Investigations at the age of $\mathbf{4 7}$ demonstrated a monoclonal IgMk paraprotein at a concentration of $4.5 \mathrm{~g} / \mathrm{l}$. Testing for antiMAG activity was negative but antisulphatide antibody activity was present at a titre of $>1 / 5000$. Screening for myeloma was negative. Review of his nerve biopsy by electron microscopy demonstrated widely spaced myelin and more recent re-examination has shown the presence of occasional endoneurial inflammatory infiltrates. DNA testing at the age of 47 revealed a chromosome 17p 11.2 duplication.

Continued neurological deterioration has taken place since the age of 47 . He was treated with high dose intravenous human immunoglobulin without benefit and also with pulsed courses of 
melphalan and later oral cyclophosphamide, again without obvious benefit. He is now aged 54 and is wheelchair bound. SAPs are absent. Peripheral nerves are inexcitable distally and proximally in the legs. He has recently developed nasopharyngeal carcinoma.

Comment: This patient has a complex clinical picture with an onset of neuropathy in childhood. This was probably responsible for the genu valgum ('bandy legs') rather than rickets. In view of the onset in early childhood this was presumably due to his HMSN la. At the age of 38 he began to experience positive sensory symptoms. These are unusual in HMSNla (Harding, Thomas, 1980) but are common in CIDP. Despite the presence of minor inflammatory infiltrates in the nerve biopsy specimen taken at the age of 44 , he showed no definite response to plasma exchange, IVIg or treatment with cyclophosphamide and possibly deteriorated with corticosteroids. Apart from the spontaneous paraesthesiae the clinical picture would be compatible either with HMSNla or an IgM paraproteinaemic neuropathy in both of which a distal motor and sensory neuropathy with prominent upper limb postural tremor may develop although the severity of the motor nerve conduction abnormality in the upper limbs would be unexpected for an IgM paraproteinaemic neuropathy. The interrelationship of the various facets of this case are considered in Chapter 4. 
Table 3.1 Details of the patients and their outcome

\begin{tabular}{|c|c|c|c|c|c|}
\hline Patient & Sex, age $(y)$ & $\begin{array}{l}\text { Duration from } \\
\text { onset(y) }\end{array}$ & $\begin{array}{l}\text { Age (y) } \\
\text { at onset }\end{array}$ & Outcome & $\begin{array}{l}\text { Type of } \\
\text { paraprotein }\end{array}$ \\
\hline 1 & $F, 82$ & 9 & 72 & A & $\operatorname{IgM} \kappa$ \\
\hline 2 & $M, 60$ & 12 & 48 & A & $\operatorname{IgM} \kappa$ \\
\hline 3 & F, 72 & 15 & 57 & A & $\operatorname{IgM} \kappa$ \\
\hline 4 & $\mathrm{~F}, 81$ & 6 & 75 & A & $\operatorname{IgM} \lambda$ \\
\hline 5 & F, 83 & 16 & 67 & A & $\operatorname{IgM} \lambda$ \\
\hline 6 & $M, 77$ & 16 & 61 & A & $\operatorname{IgM} \lambda$ \\
\hline 7 & $M, 65$ & 6 & 59 & A & $\operatorname{IgM} \times$ \\
\hline 8 & $M, 70$ & 22 & 48 & A & $\operatorname{IgM} \kappa$ \\
\hline 9 & $\mathrm{M}, 72$ & 11 & 61 & A & $\operatorname{IgM} \kappa$ \\
\hline 10 & M, 75 & 12 & 60 & A & $\operatorname{IgM} \kappa$ \\
\hline 11 & M, 77 & 5 & 72 & $\mathbf{A}$ & $\operatorname{IgM} \lambda$ \\
\hline 12 & $F, 64$ & 11 & 53 & A & $\operatorname{IgM} \lambda$ \\
\hline 13 & $M, 68$ & 16 & 52 & A & $\operatorname{IgM} \lambda$ \\
\hline 14 & $\mathrm{M}, 70$ & 10 & 60 & A & $\operatorname{IgM} \kappa$ \\
\hline 15 & M, 58 & 9 & 49 & A & $\operatorname{IgM} \kappa$ \\
\hline 16 & $\mathrm{M}, 73$ & 21 & 52 & $\mathbf{A}$ & $\operatorname{IgM} \kappa$ \\
\hline 17 & $\mathrm{~F}, 47$ & 7 & 40 & A & $\operatorname{IgM} \lambda$ \\
\hline 18 & M, 54 & 26 & 28 & A & $\operatorname{IgM} \lambda$ \\
\hline 19 & $M, 63$ & 5 & 58 & A & $\operatorname{IgM} \kappa$ \\
\hline 20 & $M, 60$ & 6 & 54 & A & $\operatorname{IgM} \kappa$ \\
\hline 21 & $\mathrm{M}, 53$ & 8 & 45 & A & $\operatorname{IgM} \kappa$ \\
\hline 22 & $\mathrm{M}, 70$ & 14 & 56 & $\mathbf{A}$ & $\operatorname{IgM} \kappa$ \\
\hline 23 & $\mathrm{M}, 72$ & 18 & 54 & A & $\operatorname{IgM} \kappa$ \\
\hline 24 & $\mathrm{~F}, 63$ & 14 & 49 & A & $\operatorname{IgM} \lambda$ \\
\hline 25 & $\mathrm{M}, 90$ & 11 & 79 & D & $\operatorname{IgM} \kappa$ \\
\hline 26 & $\mathrm{M}, 73$ & 7 & 66 & D & $\operatorname{IgM} \kappa$ \\
\hline 27 & $M, 64$ & 13 & 51 & $D$ & $\operatorname{Ig} M_{\kappa}$ \\
\hline 28 & $F, 83$ & 13 & 70 & D & $\operatorname{IgM} \kappa$ \\
\hline 29 & $\mathrm{M}, 84$ & 17 & 67 & D & $\operatorname{lgM} \kappa$ \\
\hline
\end{tabular}




\begin{tabular}{|c|c|c|c|c|c|}
\hline Patient & Sex, age $(y)$ & $\begin{array}{l}\text { Duration from } \\
\text { onset(y) }\end{array}$ & $\begin{array}{l}\text { Age (y) } \\
\text { at onset }\end{array}$ & Outcome & $\begin{array}{l}\text { Type of } \\
\text { paraprotein }\end{array}$ \\
\hline 30 & $\mathrm{M}, 78$ & 20 & 58 & $\mathrm{D}$ & $\operatorname{Ig} M x$ \\
\hline 31 & M, 75 & 24 & 51 & D & $\operatorname{IgM} \kappa$ \\
\hline 32 & $\mathrm{M}, 82$ & 5 & 77 & D & $\operatorname{IgM} \lambda$ \\
\hline 33 & $M, 60$ & 17 & 43 & $\mathrm{D}$ & $\operatorname{IgM} \lambda$ \\
\hline 34 & M, 67 & 25 & 42 & D & $\operatorname{IgM} \lambda$ \\
\hline 35 & F, 86 & 35 & 51 & D & $\operatorname{IgM} \lambda$ \\
\hline 36 & $M, 75$ & 10 & 65 & D & IgM,? \\
\hline 37 & M, 74 & 11 & 63 & D & IgM, ? \\
\hline 38 & $\mathrm{M}, 54$ & 15 & 39 & A & $\operatorname{IgM} \kappa$ \\
\hline 39 & M, 70 & 10 & 60 & A & IgG $x$ \\
\hline 40 & $F, 84$ & 14 & 70 & A & IgG $x$ \\
\hline 41 & $F, 75$ & 26 & 49 & A & $\operatorname{IgG} x$ \\
\hline 42 & $\mathrm{~F}, 60$ & 16 & 44 & A & $\operatorname{IgG} \kappa$ \\
\hline 43 & M, 79 & 18 & 61 & A & IgG $\kappa$ \\
\hline 44 & $M, 67$ & 5 & 62 & A & $\operatorname{IgG} \lambda$ \\
\hline 45 & $M, 47$ & 8 & 39 & A & $\operatorname{IgG} \lambda$ \\
\hline 46 & $\mathrm{M}, 70$ & 9 & 61 & A & $\operatorname{IgG} \lambda$ \\
\hline 47 & $\mathrm{M}, 70$ & 14 & 56 & A & $\operatorname{IgG} \lambda$ \\
\hline 48 & $F, 91$ & 26 & 65 & D & $\operatorname{IgG} \kappa$ \\
\hline 49 & $F, 73$ & 11 & 62 & $\mathrm{D}$ & $\operatorname{IgG} \lambda$ \\
\hline 50 & $\mathrm{M}, 70$ & 18 & 52 & A & $\operatorname{IgA} \lambda$ \\
\hline 51 & $\mathrm{M}, 72$ & 7 & 65 & A & - \\
\hline 52 & $\mathrm{M}, 32$ & 10 & 22 & A & - \\
\hline 53 & $M, 55$ & 41 & 13 & A & - \\
\hline 54 & M, 29 & 15 & 14 & A & - \\
\hline 55 & $M, 67$ & 15 & 52 & A & - \\
\hline
\end{tabular}

y: years; M: male; F: female; D: deceased; A: alive; ?: paraprotein light chain unknown. 
Table 3.2 Cause of death

a) Haematological malignancy

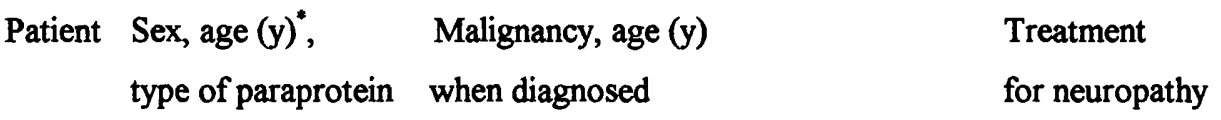

$33 \mathrm{M}, 43 / 60, \operatorname{IgM} \lambda \quad$ Acute myeloid leukaemia, $60 \quad$ Prednisolone and

azathioprine for the

last $2 \mathrm{y}$.

$34 \mathrm{M}, 42 / 67, \operatorname{IgM} \lambda \quad$ Waldenström macroglobulinaemia,65 Intermittent

chlorambucil at age

64 for $2 \mathrm{y}$.

$49 \quad \mathrm{~F}, 62 / 73, \operatorname{IgG} \lambda \quad$ Refractory anaemia with

Prednisolone and

excess blasts (RAEB), 73

azathioprine for $20 \mathrm{mo}$

then melphalan for $5 y$

with another course

given 4 months

prior to the diagnosis

of RAEB.

b) Non haematological malignancy

\begin{tabular}{|c|c|c|c|}
\hline Patient & $\begin{array}{l}\text { Sex, age }(y)^{*} \\
\text { type of paraprotein }\end{array}$ & $\begin{array}{l}\text { Malignancy, age (y) } \\
\text { when diagnosed }\end{array}$ & $\begin{array}{l}\text { Treatment } \\
\text { for neuropathy }\end{array}$ \\
\hline
\end{tabular}

27 M, 51/64, IgM $\kappa \quad$ Adenocarcinoma of the colon, Started age 61 with $64 \quad$ prednisolone $(3 \mathrm{y})$ and

chlorambucil (18 mo).

28 F, 70/83, IgM $\kappa \quad$ Liver metastasis of unknown primary On melphalan from

8370 for $6 y$.

$30 \mathrm{M}, 58 / 78, \operatorname{IgM} \kappa \quad$ Carcinoma of the pancreas, $77 \quad 6$ courses of

cyclophosphamide

at age 68.

$36 \mathrm{M}, 65 / 75, \operatorname{IgM}$ ? Carcinoma of the pharynx,? Prednisolone for the

last $6 \mathrm{y}$.

$9 \mathrm{M}, 61 / 72^{* *}$, IgMk Carcinoma of the rectum, 68 None

16 M, 52/73**, IgM $\kappa$ Carcinoma of the stomach, $72 \quad$ None

$\mathrm{y}^{*}$ : age (in years) at onset of neuropathic symptoms/ age at the time of death except **;

?: unknown age when malignancy diagnosed; ${ }^{* *}$ : patient still alive 
Table 3.3 Clinical and immunological data for patients with IgM paraproteinaemic neuropathy

\begin{tabular}{|c|c|c|c|c|}
\hline Patient & $\begin{array}{c}\text { Paraprotein } \\
\text { type }\end{array}$ & $\begin{array}{l}\text { Paraprotein } \\
\text { concentration* }\end{array}$ & $\begin{array}{l}\text { Antibody } \\
\text { activity }\end{array}$ & Neuropathy \\
\hline
\end{tabular}

(g/)

\begin{tabular}{|c|c|c|c|c|}
\hline 1 & IgMk & $\#$ & anti-S & $\mathbf{S}>\mathbf{M}, \mathbf{1}>\mathbf{S}$ \\
\hline 2 & IgMk & 9.0 & anti-S & $\mathrm{S}=\mathrm{M}, \mathrm{l}=\mathrm{S}$ \\
\hline 3 & IgMk & 10.0 & anti-S & $\mathrm{S}>\mathrm{M}, \mathrm{I}>\mathrm{S}$ \\
\hline 4 & $\operatorname{IgM} \lambda$ & 6.5 & anti-S & $\mathbf{S}>\mathrm{M}, \mathrm{l}>\mathrm{S}$ \\
\hline 5 & $\operatorname{IgM} \lambda$ & 7.6 & anti-S & $\mathbf{S}>\mathrm{M}, \mathbf{l}>\mathbf{S}$ \\
\hline 6 & $\operatorname{Ig} M \lambda$ & 6.0 & anti-S & $\mathrm{S}>\mathrm{M}, \mathbf{I}>\mathbf{S}$ \\
\hline 7 & IgMk & 2.6 & antiMAG & $\mathrm{S}=\mathrm{M}, \mathrm{I}>\mathrm{S}$ \\
\hline 8 & IgMk & 10.4 & antiMAG & $\mathbf{S}>\mathbf{M}, 1>\mathbf{S}$ \\
\hline 9 & IgMk & 6.8 & antiMAG & $\mathrm{S}=\mathrm{M}, \mathrm{l}>\mathrm{S}$ \\
\hline 10 & IgMk & 8.4 & antiMAG & $\mathrm{S}>\mathrm{M}, 1>\mathrm{s}$ \\
\hline 11 & $\operatorname{IgM} \lambda \lambda$ & 6.4 & antiMAG & $\mathrm{S}=\mathrm{M}, 1>\mathrm{S}$ \\
\hline 12 & $\operatorname{IgM} \lambda$ & 6.0 & antiMAG & $\mathrm{S}=\mathrm{M}, \mathrm{l}>\mathrm{S}$ \\
\hline 13 & $\operatorname{IgM} \lambda$ & 3.3 & antiMAG & $\mathrm{S}=\mathrm{M}, \mathrm{l}>\mathrm{S}$ \\
\hline 14 & $\operatorname{IgMk}$ & 11.1 & antiMAG \& S & $\mathrm{S}=\mathrm{M}, \mathrm{l}=\mathrm{s}$ \\
\hline 15 & IgMk & 8.0 & antiMAG \& S & $\mathrm{S}=\mathrm{M}, \mathrm{l}=\mathrm{s}$ \\
\hline 16 & IgMk & biclonal & antiMAG \& S & $\mathbf{S}>\mathbf{M}, \mathbf{1}>\mathbf{S}$ \\
\hline 17 & $\operatorname{IgM} \lambda$ & 4.5 & antidisialosyl & $\mathbf{S}>\mathbf{M}, 1>\mathbf{s}$ \\
\hline 18 & $\operatorname{IgM} \lambda$ & triclonal & antidisialosyl & $\mathbf{S}>\mathbf{M}, \mathbf{l}>\mathbf{s}$ \\
\hline 19 & IgMk & 4.1 & - & $\mathbf{S}>\mathbf{M}, \mathbf{l}>\mathbf{S}$ \\
\hline 20 & IgMk & 4.4 & - & $\mathrm{S}=\mathrm{M}, \mathrm{l}=\mathrm{s}$ \\
\hline 21 & IgMk & 4.7 & - & $\mathbf{S}>\mathbf{M}, \mathbf{l}>\mathbf{S}$ \\
\hline 22 & IgMk & 1.9 & - & $\mathbf{S}>\mathbf{M}, \mathbf{l}>\mathbf{S}$ \\
\hline 23 & IgMk & 5.3 & - & $\mathbf{S}>\mathbf{M}, 1>\mathbf{s}$ \\
\hline 24 & $\operatorname{Ig} M \lambda$ & triclonal & - & $\mathrm{S}=\mathrm{M}, \mathrm{l}=\mathbf{s}$ \\
\hline
\end{tabular}

*:paraprotein level from last follow-up; \#:paraprotein quantification not done; S:sensory; M:motor; 1:large fibre; s:small fibre; -: not detected. 
Table 3.4 Clinical and immunological data for patients with IgG/A

paraproteinaemic neuropathy

\begin{tabular}{|c|c|c|c|c|}
\hline Patient & $\begin{array}{l}\text { Paraprotein } \\
\text { type }\end{array}$ & $\begin{array}{l}\text { Paraprotein } \\
\text { concentration } \\
(\mathrm{g} / \mathrm{l})\end{array}$ & $\begin{array}{l}\text { Antibody } \\
\text { activity }\end{array}$ & Neuropath \\
\hline 39 & IgGK & 6.4 & - & $\dagger$ \\
\hline 40 & IgGK & 10.2 & - & $\mathrm{S}>\mathrm{M}, 1>\mathbf{S}$ \\
\hline 41 & IgGK & biclonal & - & $\mathrm{S}=\mathrm{M}, 1>\mathbf{S}$ \\
\hline 42 & $\mathrm{IgGK}$ & 3.5 & - & $\mathrm{S}>\mathrm{M}, \mathrm{l}>\mathrm{s}$ \\
\hline 43 & $\mathrm{IgGK}$ & 10.6 & - & $\mathbf{S}>\mathbf{M}, \mathbf{l}>\mathbf{s}$ \\
\hline 44 & $\operatorname{IgG} \lambda$ & 4.2 & - & $\mathrm{S}>\mathrm{M}, \mathrm{l}=\mathrm{s}$ \\
\hline 45 & $\operatorname{IgG} \lambda$ & ND & - & $\mathrm{S}>\mathrm{M}, \mathbf{1}>\mathbf{S}$ \\
\hline 46 & $\operatorname{IgG\lambda }$ & 5.0 & - & $\mathbf{S}>\mathbf{M}, 1>\mathbf{S}$ \\
\hline 47 & $\operatorname{IgG} \lambda$ & 11.0 & - & $\mathrm{S}=\mathrm{M}, \mathrm{l}=\mathrm{s}$ \\
\hline 50 & $\operatorname{IgA\lambda }$ & ND & - & $\mathrm{S}=\mathrm{M}, \mathrm{l}=\mathrm{s}$ \\
\hline
\end{tabular}

For abbreviations, see footnotes to Table 3.3 ; $\dagger$ :assessment not possible,see text; ND:not detected

Table 3.5 Clinical data for patients with CIDP

\begin{tabular}{lll} 
Patient & Paraprotein & Neuropathy \\
\hline 53 & - & $\mathrm{S}>\mathrm{M}, \mathrm{l}>\mathrm{s}$ \\
54 & - & $\mathrm{S}>\mathrm{M}, \mathrm{l}=\mathrm{s}$ \\
55 & - & $\mathrm{S}<\mathrm{M}, \mathrm{l}>\mathrm{s}$ \\
56 & - & $\mathrm{S}<\mathrm{M}, \mathrm{l}>\mathrm{s}$ \\
57 & - & $\mathrm{S}=\mathrm{M}, \mathrm{l}>\mathrm{s}$
\end{tabular}

For abbreviations, see footnotes to Table 3.3 
Table 3.6 Changes in sensory recordings

\begin{tabular}{|c|c|c|c|c|}
\hline & \multicolumn{2}{|c|}{ Absent UL SAPs } & \multicolumn{2}{|c|}{ Absent LL SAPs } \\
\hline & First & Last & First & Last \\
\hline \multicolumn{5}{|l|}{ 1. $\operatorname{IgM}$} \\
\hline Anti-S & 4 & 6 & 5 & 6 \\
\hline \multicolumn{5}{|l|}{$n=6 ; d=12.4 y$} \\
\hline AntiMAG & 6 & 7 & 7 & 7 \\
\hline \multicolumn{5}{|l|}{$n=7 ; d=11.8 y$} \\
\hline AntiMAG+S & 2 & 3 & 3 & 3 \\
\hline \multicolumn{5}{|l|}{$n=3 ; d=12.6 y$} \\
\hline Antidisialosyl & 1 & 2 & 2 & 2 \\
\hline \multicolumn{5}{|l|}{$n=2 ; d=16 y$} \\
\hline Unidentified & 1 & 3 & 4 & 6 \\
\hline \multicolumn{5}{|l|}{$n=6 ; d=9.8 y$} \\
\hline \multicolumn{5}{|l|}{ 2. $\lg G / A$} \\
\hline$n=10 ; d=12.7 y$ & 8 & 5 & 7 & 8 \\
\hline \multicolumn{5}{|l|}{ 3. CIDP } \\
\hline$n=5, d=12 y$ & 1 & 1 & 2 & 1 \\
\hline
\end{tabular}

UL: upper limb(s); SAPs: sensory action potentials; LL: lower limb(s); $n:$ umber; d:duration from the onset of neurological symptoms; $y$ : year; 
Table 3.7 Changes in MCV

No change $\begin{aligned} & \text { UL } \\ & \text { N }\end{aligned} \quad \downarrow \quad$ No change $\quad \uparrow \quad \downarrow$

1. $\lg M$

Anti-S

$3-3$

6 (4\#)

$\mathrm{n}=6, \mathrm{~d}=12.4 \mathrm{y}$

AntiMAG

$4 \quad-\quad 3$

7\#

$\mathrm{n}=7, \mathrm{~d}=11.8 \mathrm{y}$

AntiMAG+S

$1 \quad-\quad 2$

2\#

1\#

$\mathrm{n}=3, \mathrm{~d}=12.6 \mathrm{y}$

Antidisialosyl

$1 \quad-\quad 1$

1

1

$\mathrm{n}=2, \mathrm{~d}=16 \mathrm{y}$

Unidentified

$1 \quad-\quad 5$

3

3\#

$n=6, d=9.8 y$

2. $\lg G / A$

$n=10, d=12.7 y$

$\begin{array}{lll}3 & 4 & 3\end{array}$

$5(4 \#) \quad 1$

4 (3\#)

1. CIDP

$n=5, d=12 y$

122

2

$3(2 \#)$

For abbreviations, see footnotes to Table 3 ; $\uparrow:$ improvement; $\downarrow$ : deterioration; \#:number of nerves with no recordable potentials 
Table 3.8.1 Neurophysiological findings* at the final follow-up

\begin{tabular}{|c|c|c|c|c|}
\hline & \multirow{2}{*}{$\begin{array}{l}\text { Upper limb } \\
M C V(m / s)\end{array}$} & \multicolumn{2}{|c|}{$\mathrm{DML}(\mathrm{ms})$} & \multirow{2}{*}{$\begin{array}{l}\text { dCMAP } \\
\text { amplitude (mV) }\end{array}$} \\
\hline & & Median & Ulnar & \\
\hline \multicolumn{5}{|l|}{$1 \lg M$} \\
\hline \multicolumn{5}{|l|}{ Anti-S } \\
\hline Axonal & $\begin{array}{l}54 \\
n=1\end{array}$ & - & 3.1 & 3.4 \\
\hline Demyelinating & $\begin{array}{l}21 \pm 11 \\
(13-36) \\
n=5\end{array}$ & $\begin{array}{l}8.3 \pm 2.3 \\
(6.8-11.7) \\
n=4\end{array}$ & $\begin{array}{l}9.4 \pm 7.6 \\
(3.2-18) \\
n=3\end{array}$ & $\begin{array}{l}5.9 \pm 1.3 \\
(5-6.8) \\
n=2\end{array}$ \\
\hline \multicolumn{5}{|l|}{ AntiMAG } \\
\hline Demyelinating & $\begin{array}{l}17 \pm 12 \\
(2.7-38) \\
n=7\end{array}$ & $\begin{array}{l}8.5 \pm 0.7 \\
(8.8-9) \\
n=2\end{array}$ & $\begin{array}{l}16.1 \pm 10.4 \\
(5.3-36) \\
n=6\end{array}$ & $\begin{array}{l}1.4 \pm 1.4^{\star \star} \\
(0.2-3.6) \\
n=7\end{array}$ \\
\hline \multicolumn{5}{|l|}{ AntiMAG+S } \\
\hline Demyelinating & $\begin{array}{l}25 \pm 9 \\
(18-35) \\
n=3\end{array}$ & $\begin{array}{l}15.4 \pm 6.6 \\
(10.7-20) \\
n=2\end{array}$ & $\begin{array}{l}8.5 \pm 2.4 \\
(5.7-10) \\
n=3\end{array}$ & $\begin{array}{l}6.2 \pm 3.7 \\
(2-6.8) \\
n=3\end{array}$ \\
\hline \multicolumn{5}{|l|}{ Antidisialosyl } \\
\hline Demyelinating & $\begin{array}{l}31 \pm 5 \\
(27-34) \\
n=2\end{array}$ & $\begin{array}{l}6.8 \pm 2.9 \\
(4.7-8.8) \\
n=2\end{array}$ & $\begin{array}{l}5.4 \pm 2.3 \\
(3.7-7) \\
n=2\end{array}$ & $\begin{array}{l}6 \pm 2.8 \\
(4-8) \\
n=2\end{array}$ \\
\hline \multicolumn{5}{|l|}{ Unidentified } \\
\hline Axonal & $\begin{array}{l}40 \\
n=1\end{array}$ & 3.2 & - & 7.5 \\
\hline Demyelinating & $\begin{array}{l}26 \pm 6 \\
(21-38) \\
n=5\end{array}$ & $\begin{array}{l}8.5 \pm 1.8 \\
(5.5-9.8) \\
n=5\end{array}$ & $\begin{array}{l}5.1 \pm 0.8 \\
(4.5-5.7) \\
n=2\end{array}$ & $\begin{array}{l}6.4 \pm 3.8 \\
(2.3-12.5) \\
n=5\end{array}$ \\
\hline \multicolumn{5}{|l|}{$2 \quad \mathrm{Ig} G / \mathrm{A}$} \\
\hline Demyelinating & $\begin{array}{l}25 \pm 10 \\
(11-40) \\
n=10\end{array}$ & $\begin{array}{l}11.5 \pm 8.1 \\
(5.5-28.7) \\
n=8\end{array}$ & $\begin{array}{l}5 \pm 0.9 \\
(2.2-3.8) \\
n=4\end{array}$ & $\begin{array}{l}4.5 \pm 3.9 \\
(0.2-12) \\
n=10\end{array}$ \\
\hline \multicolumn{5}{|l|}{ 3. CIDP } \\
\hline Demyelinating & $\begin{array}{l}33.8 \pm 15 \\
(13-48) \\
n=5\end{array}$ & $\begin{array}{l}5.2 \pm 0.5 \\
(4.7-5.7) \\
n=3\end{array}$ & $\begin{array}{l}6.1 \pm 2.6 \\
(4.5-7.7) \\
n=2\end{array}$ & $\begin{array}{l}6.0 \pm 3.9 \\
(1.5-11) \\
n=5\end{array}$ \\
\hline
\end{tabular}


Table 3.8.2 Neurophysiological findings" at the final follow-up

\begin{tabular}{|c|c|c|c|c|}
\hline & \multicolumn{2}{|c|}{ Residual latency } & \multicolumn{2}{|c|}{ Terminal latency index } \\
\hline & $\begin{array}{l}\text { Median } \\
(1.97 \pm 0.27)\end{array}$ & $\begin{array}{l}\text { Ulnar } \\
(\mathbf{1 . 2 \pm 0 . 1 )}\end{array}$ & $\begin{array}{l}\text { Median } \\
(0.433 \pm 0.045)\end{array}$ & $\begin{array}{l}\text { Ulnar } \\
(0.492 \pm 0.75)\end{array}$ \\
\hline \multicolumn{5}{|l|}{ 1.IgM } \\
\hline \multicolumn{5}{|l|}{ Anti-S } \\
\hline Axonal & $\begin{array}{l}- \\
n=1\end{array}$ & 1.9 & - & 1.9 \\
\hline Demyelinating & $\begin{array}{l}7.4 \pm 3.4 \\
(5.1-11.3) \\
n=3\end{array}$ & $\begin{array}{l}5.5 \pm 2.9 \\
(3.5-8.8) \\
n=3\end{array}$ & $\begin{array}{l}0.303 \pm 0.084 \\
(0.25-0.4) \\
n=3\end{array}$ & $\begin{array}{l}0.45 \pm 0.046 \\
(0.4-0.5) \\
n=3\end{array}$ \\
\hline \multicolumn{5}{|l|}{ AntiMAG } \\
\hline Demyelinating & $\begin{array}{l}8.5 \pm 1.8 \\
(7.3-10.5) \\
n=3\end{array}$ & $\begin{array}{l}9.4 \pm 3.4 * * \\
(5-13.5) \\
n=5\end{array}$ & $\begin{array}{l}0.31 \pm 0.167 \\
(0.16-0.49) \\
n=3\end{array}$ & $\begin{array}{l}0.453 \pm 0.112 \\
(0.41-0.62) \\
\mathrm{n}=5\end{array}$ \\
\hline \multicolumn{5}{|l|}{$A n t i M A G+S$} \\
\hline Demyelinating & $\begin{array}{l}13.3 \pm 5.6 \\
(9.3-17.2) \\
n=2\end{array}$ & $\begin{array}{l}6.4 \pm 1.8 \\
(5.1-7.7) \\
n=2\end{array}$ & $\begin{array}{l}0.135 \pm 0.042 \\
(0.132-0.138) \\
n=2\end{array}$ & $\begin{array}{l}0.335 \pm 0.191 \\
(0.2-0.47) \\
\mathrm{n}=2\end{array}$ \\
\hline \multicolumn{5}{|l|}{ Antidisialosyl } \\
\hline Demyelinating & $\begin{array}{l}4.9 \pm 2.4 \\
(3.2-6.6) \\
n=2\end{array}$ & $\begin{array}{l}4.25 \\
n=1\end{array}$ & $\begin{array}{l}0.282 \pm 0.042 \\
(0.25-0.312) \\
n=2\end{array}$ & $\begin{array}{l}0.39 \\
n=1\end{array}$ \\
\hline \multicolumn{5}{|l|}{ Unidentified } \\
\hline Axonal & $\begin{array}{l}1.79 \\
n=1\end{array}$ & - & 0.44 & - \\
\hline Demyelinating & $\begin{array}{l}6.2 \pm 2 \\
(3.3-8.4) \\
n=5\end{array}$ & $\begin{array}{l}3.7 \pm 0.7 \\
(3.2-4.2) \\
n=2\end{array}$ & $\begin{array}{l}0.287 \pm 0.0926 \\
(0.15-0.44) \\
n=5\end{array}$ & $\begin{array}{l}0.145 \pm 0.163 \\
(0.029-0.26) \\
\mathrm{n}=2\end{array}$ \\
\hline
\end{tabular}

2 IgG/A

$\begin{array}{llll}7.78 \pm 7.1 & 3.12 \pm 1.66 & 0.306 \pm 0.138 & 0.368 \pm 0.122 \\ (2.9-25) & (1.3-5) & (0.11-0.51) & (0.27-0.51) \\ \mathrm{n}=10 & \mathrm{n}=4 & \mathrm{n}=10 & \mathrm{n}=4\end{array}$

3.CIDP

$\begin{array}{llll}4.243 \pm 1.1 & 2.984 \pm 0.14 & 0.323 \pm 0.1 & 0.48 \pm 0.17 \\ (3.423-5.649) & (2.882-3.085) & (0.219-0.452) & (0.6-0.359) \\ \mathrm{n}=4 & \mathrm{n}=2 & \mathrm{n}=4 & \mathrm{n}=2\end{array}$

For abbreviations, see footnotes to Table 3.8.1; normal values in bold. 
Table 3.9 Relationship between upper limb distal motor latency and forearm conduction velocity for different paraproteins: slope coefficients and significance levels

$\begin{array}{lllll}\text { Paraprotein } & \text { n } & \text { First assessment } & & \text { Final assessment } \\ \text { reactivity } & & \text { Slope coefficient } & P \text { value } & \text { Slope coefficient } P \text { value }\end{array}$

IgM

$\begin{array}{lccccc}\text { Anti-S } & 5 & -0.40 & 0.04 & -0.36 & 0.07 \\ \text { AntiMAG } & 7 & -0.58 & 0.03 & -0.53 & 0.10 \\ \text { Unidentified } & 5 & -0.37 & 0.06 & 0.05 & 0.74 \\ & & & & & \\ \text { IgG } & 9 & -0.11 & 0.54 & -0.36 & 0.25 \\ & & & & & \\ \text { CIDP } & 5 & -0.08 & 0.34 & -0.06 & 0.19\end{array}$

$\mathrm{n}=$ number of patients 
Table 3.10 MCV obtained by magnetic stimulation

\begin{tabular}{llll} 
Patient & $\begin{array}{l}\text { MCV } \\
\text { elbow-wrist } \\
(\mathrm{m} / \mathrm{s})\end{array}$ & $\begin{array}{l}\text { MCV } \\
\text { axilla-elbow } \\
(\mathrm{m} / \mathrm{s})\end{array}$ & $\begin{array}{l}\text { MCV } \\
\text { C7-axilla } \\
(\mathrm{m} / \mathrm{s})\end{array}$ \\
\hline Case 13 & 35 & 29 & 29 \\
Case 28 & 16 & 18 & 21 \\
Case 42 & 28 & 17 & 40 \\
Case 43 & 32 & 28 & 55 \\
\hline
\end{tabular}

Table 3.11 Nerve biopsy findings

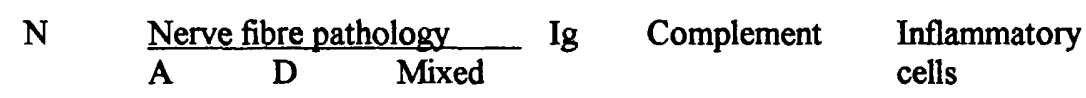

$\begin{array}{llllllll}\text { IgM } & & & & & 3 & 2 & 1 \\ \text { Anti-S } & 4 & - & 4 & - & 3 & 2 & - \\ \text { AntiMAG } & 4 & - & 4 & - & 4 & 3 & 1 \\ \text { AntiMAG+S } & 3 & - & 3 & - & 2 & 1 & - \\ \text { Antidisialosyl } & 2 & - & 2 & - & - & - & 1 \\ \text { Unidentified } & 3 & - & 2 & 1 & 1 & - & - \\ \text { Unclassified } & 12 & - & 4 & 8 & 9 & 6 & - \\ \text { IgG/A } & 6 & - & 2 & 4 & - & - & - \\ \text { CIDP } & 2 & - & - & 2 & - & - & \\ & & & & & & & \end{array}$


Fig. 3.1

Kaplan - Meier survival curves between patients for different comparisons 
Figure 3.1.1a Male : female

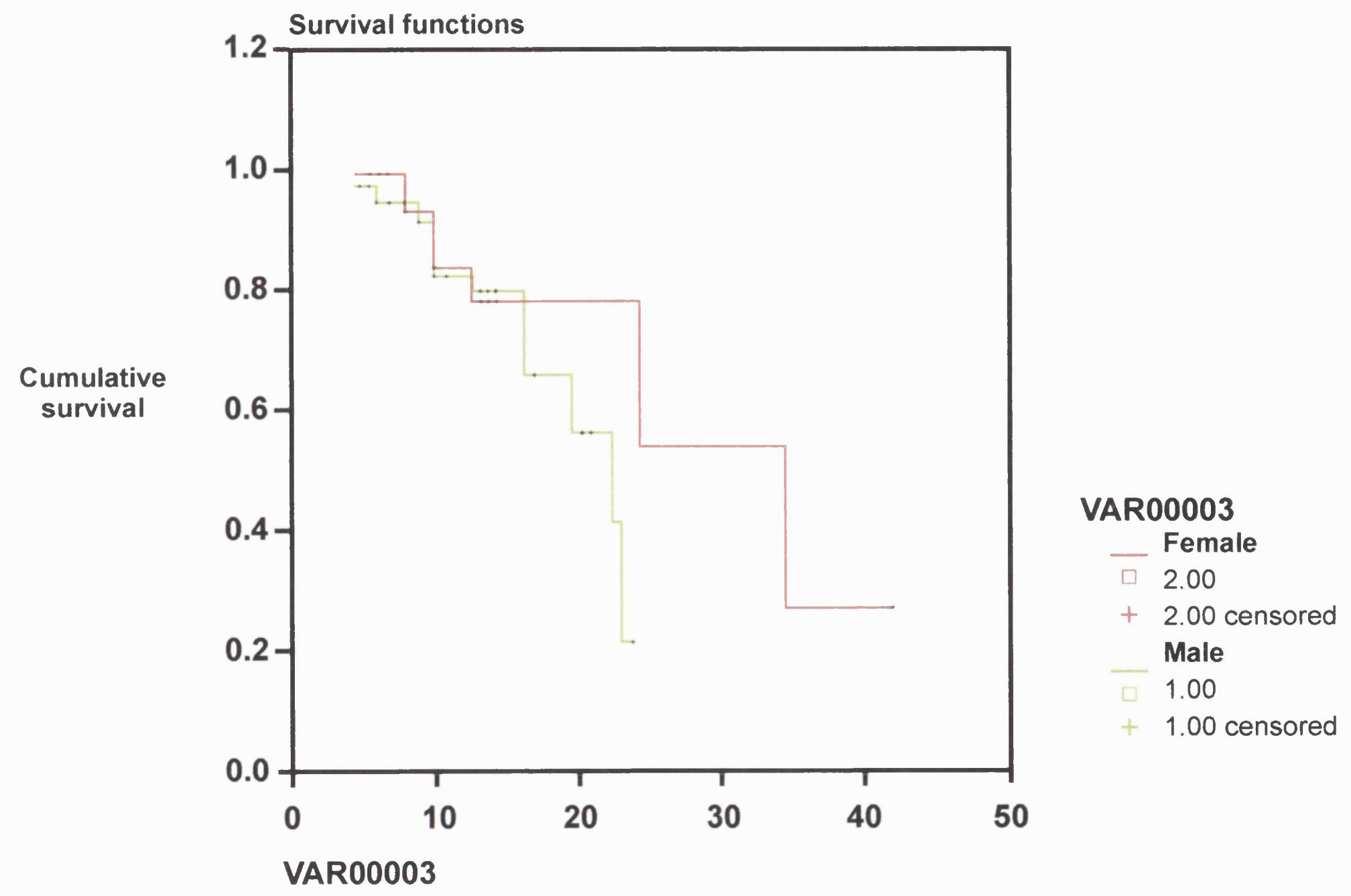


Figure 3.1.1b IgM paraproteins : other groups

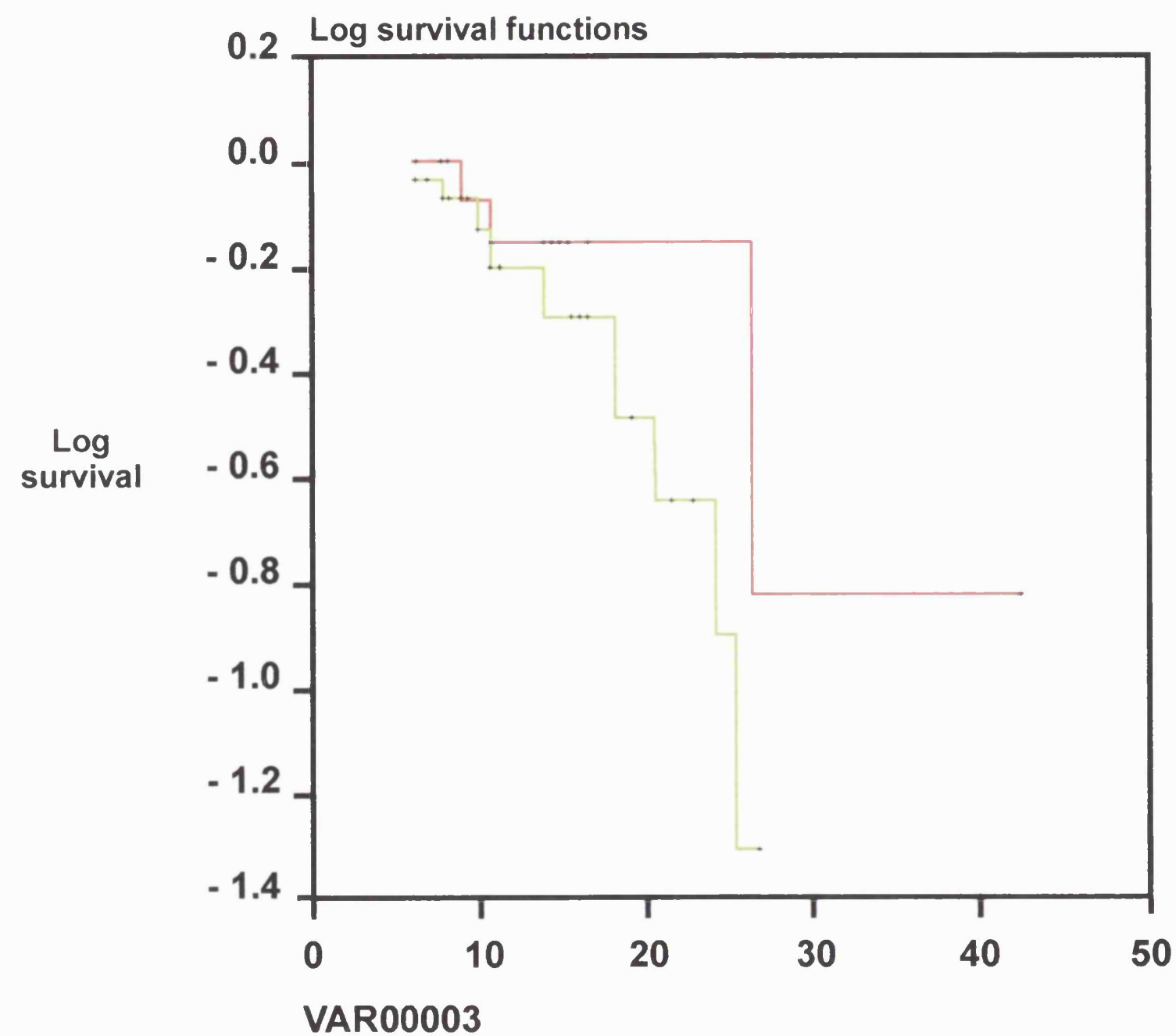

\section{VAR00005}

_ Other groups

- 2.00

+2.00 censored IgM paraproteins

1.00

1.00 censored 
Figure 3.1.1c Degrees of disability

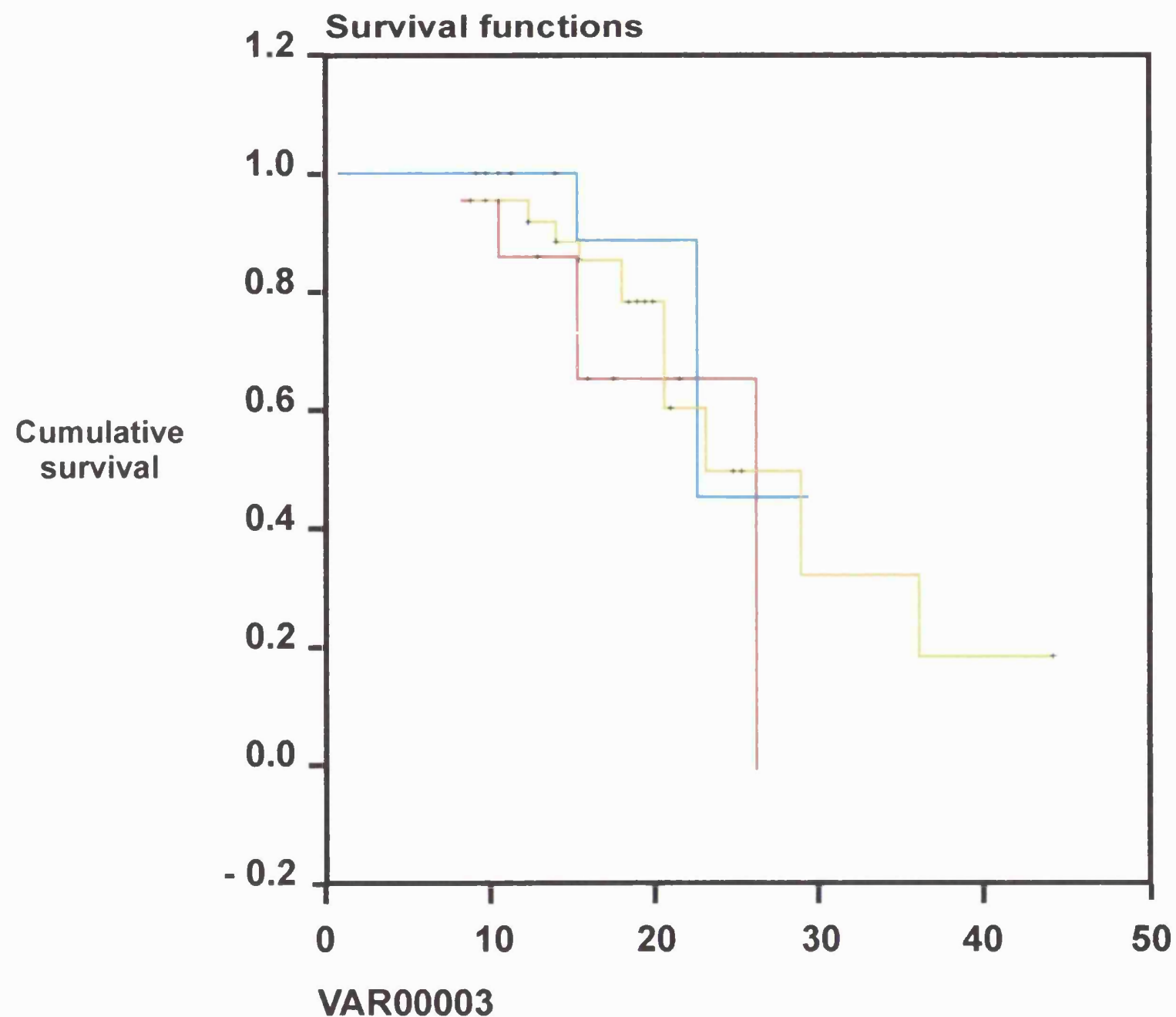

VAR00010

4.00

+4.00 censored

3.00

3.00 censored

2.00

2.00 censored

1.00

1.00 censored 
Figure 3.1.1d Type of nerve conduction abnormality

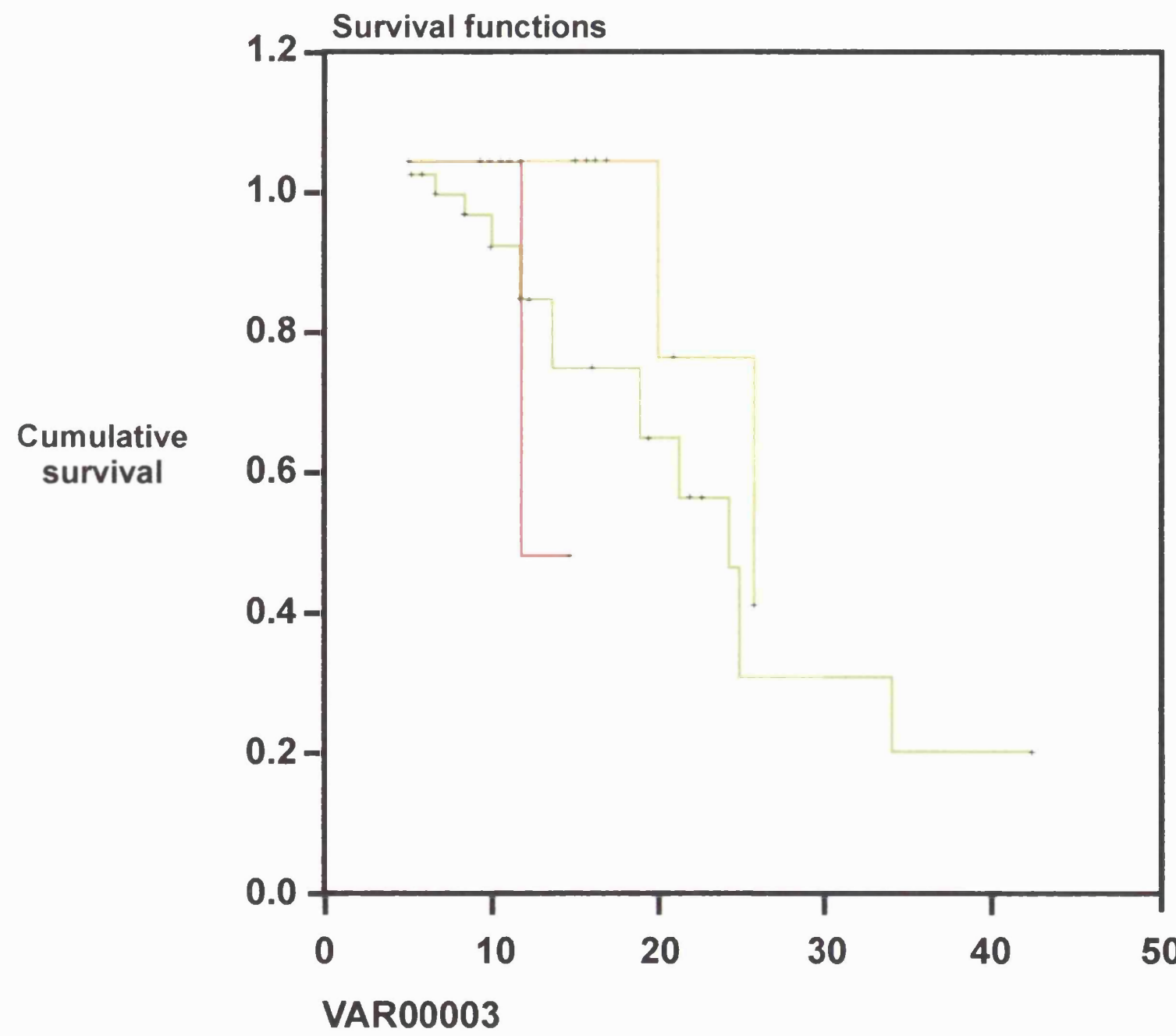

\section{VAR00011}

multifocal abnormalities

3.00

3.00 censored

axonal degeneration

2.00

2.00 censored

demyelination

1.00

1.00 censored 
Figure 3.1.2 Cumulative survival for all paraproteinaemic patients

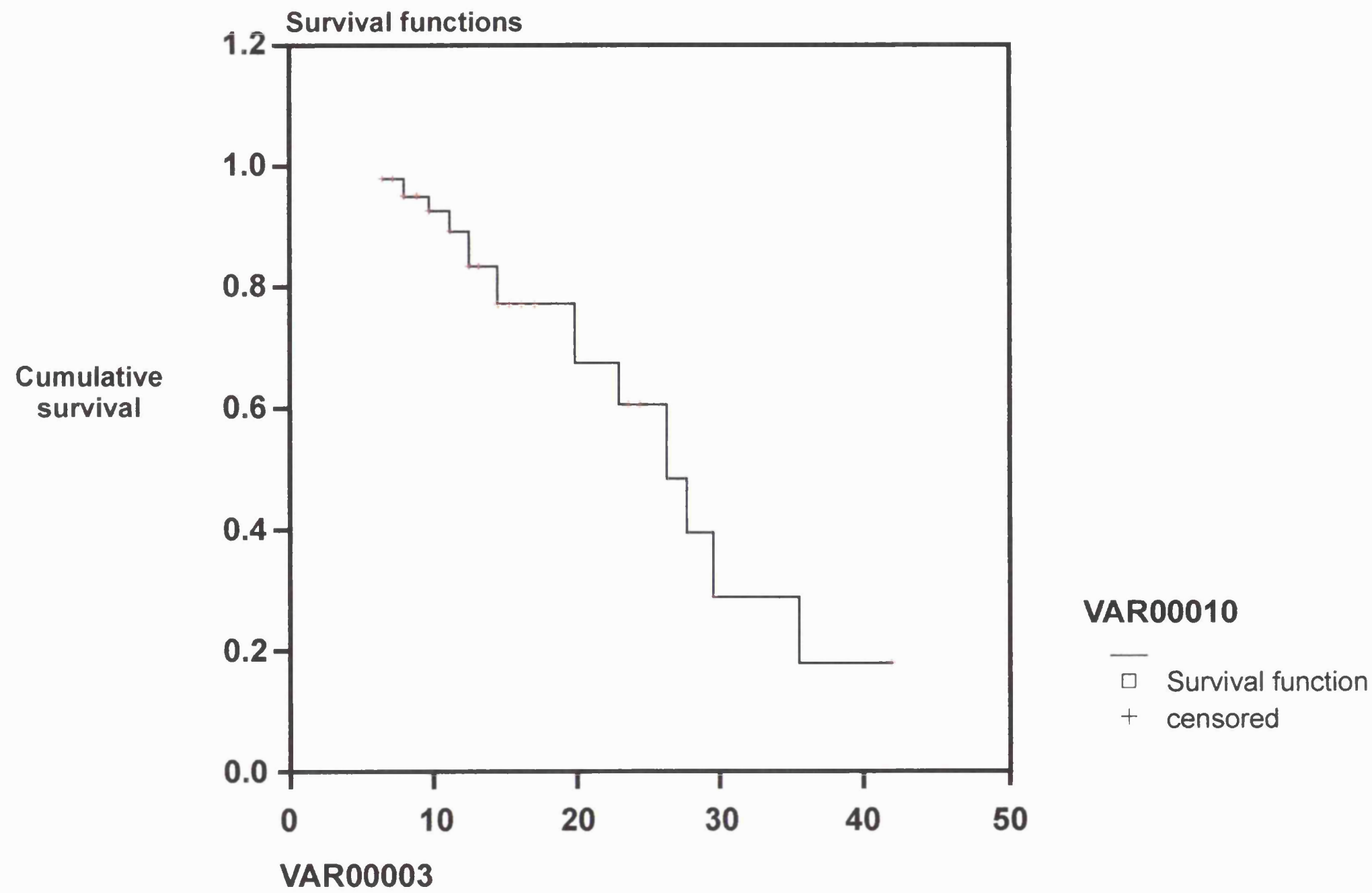


Fig. 3.2

Comparison of scores ${ }^{*}$ between the first and last visits for $\lg M, \lg G / A$ and CIDP.

\# summed scores, means \pm S.D. with range in parenthesis.

$P$ : significant difference in values between groups of patients either at time of initial or final examination. 


\section{Fig 3.2.1 Symptoms}

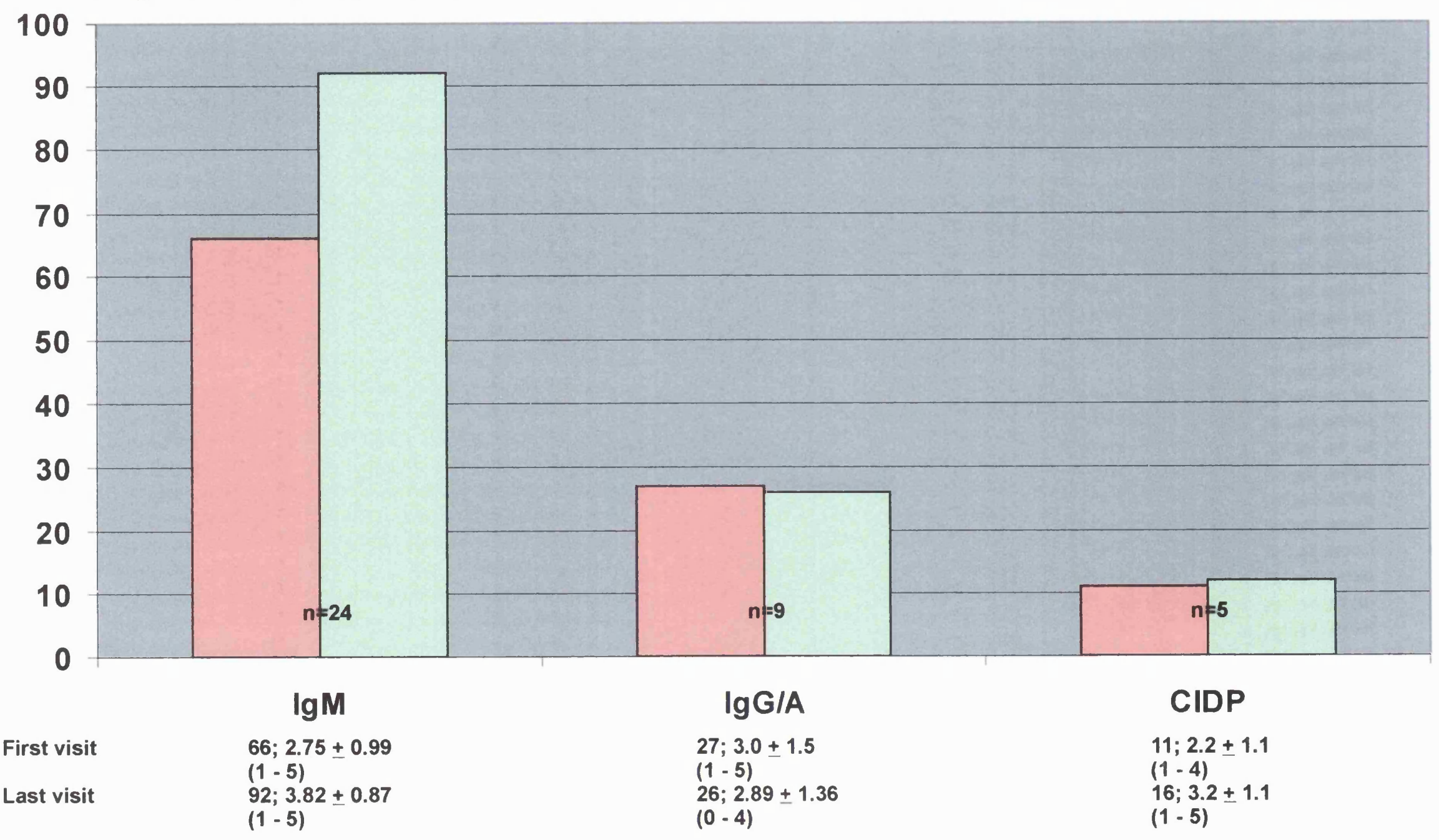




\section{Fig 3.2.2 Muscle strength}

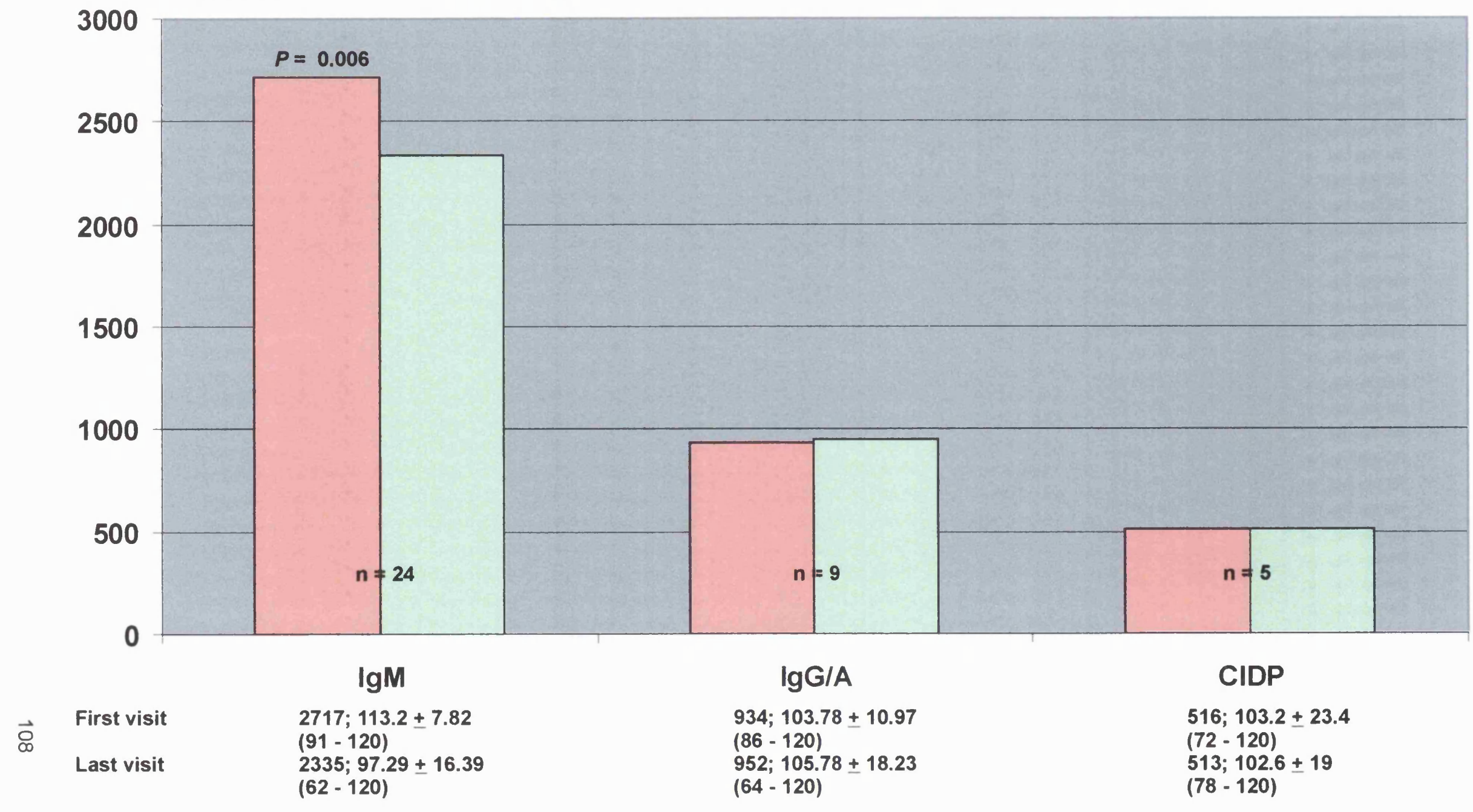


Fig 3.2.3 Tendon reflexes

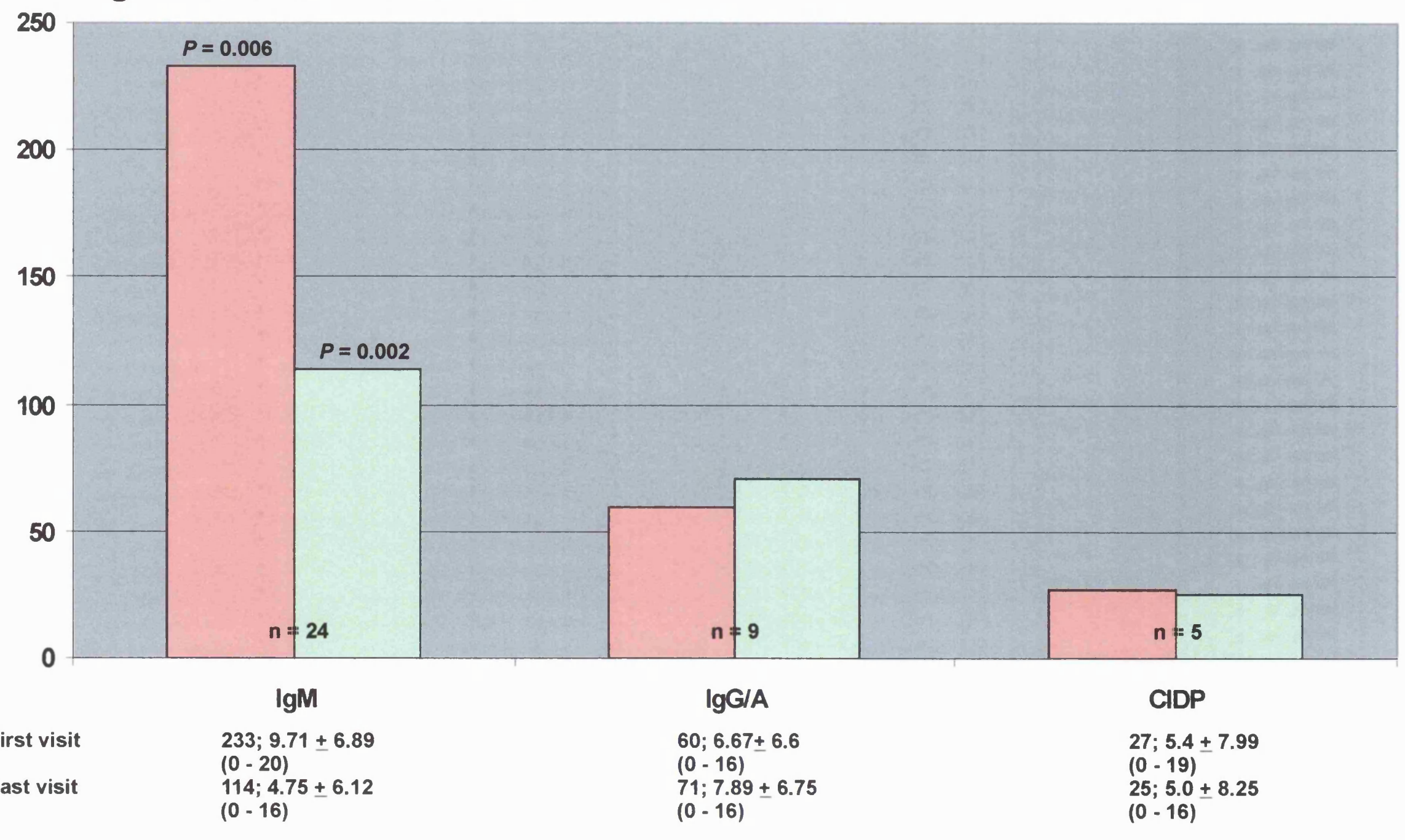


Fig 3.2.4 Light touch

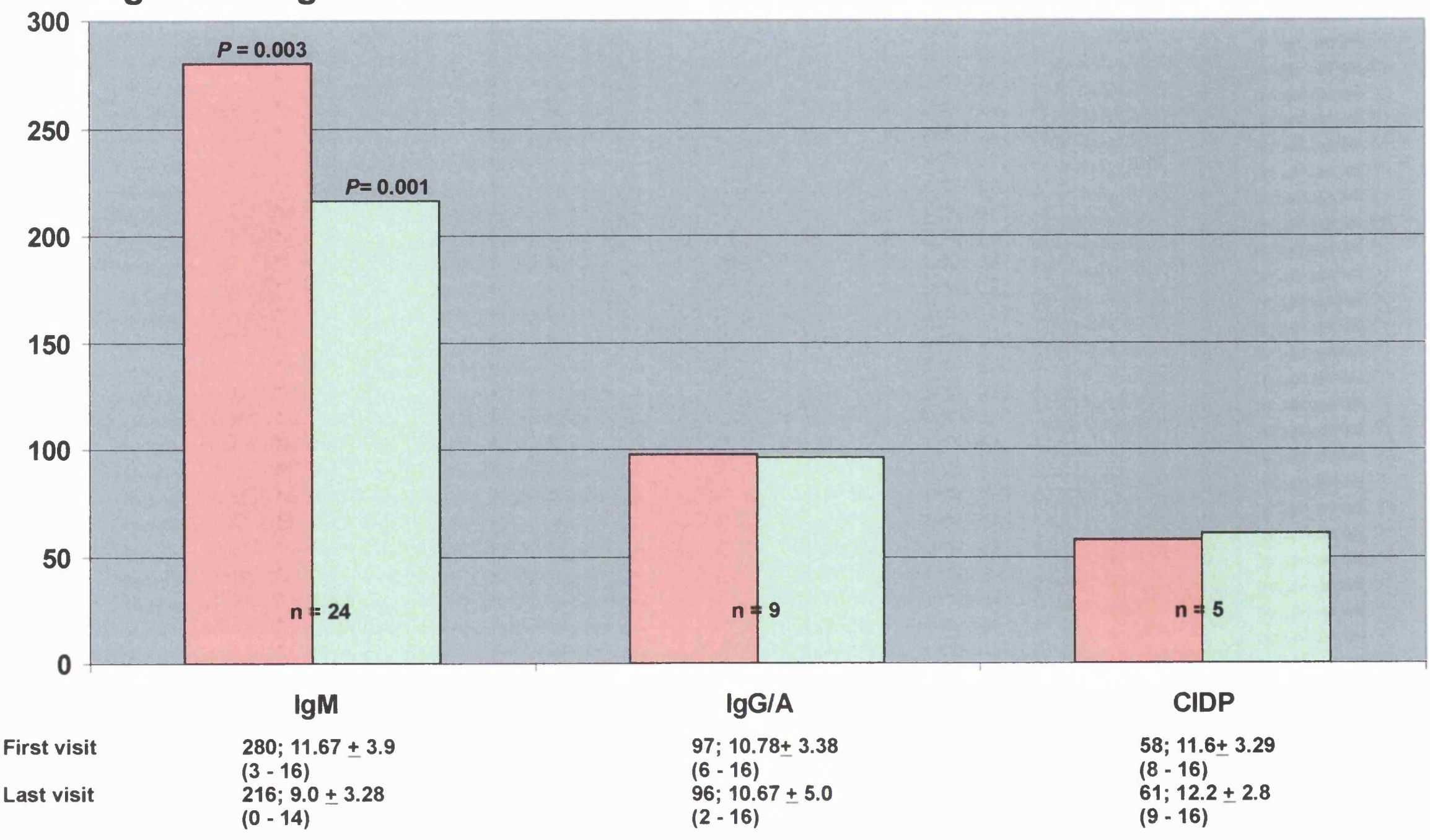


Fig 3.2.5 Pinprick

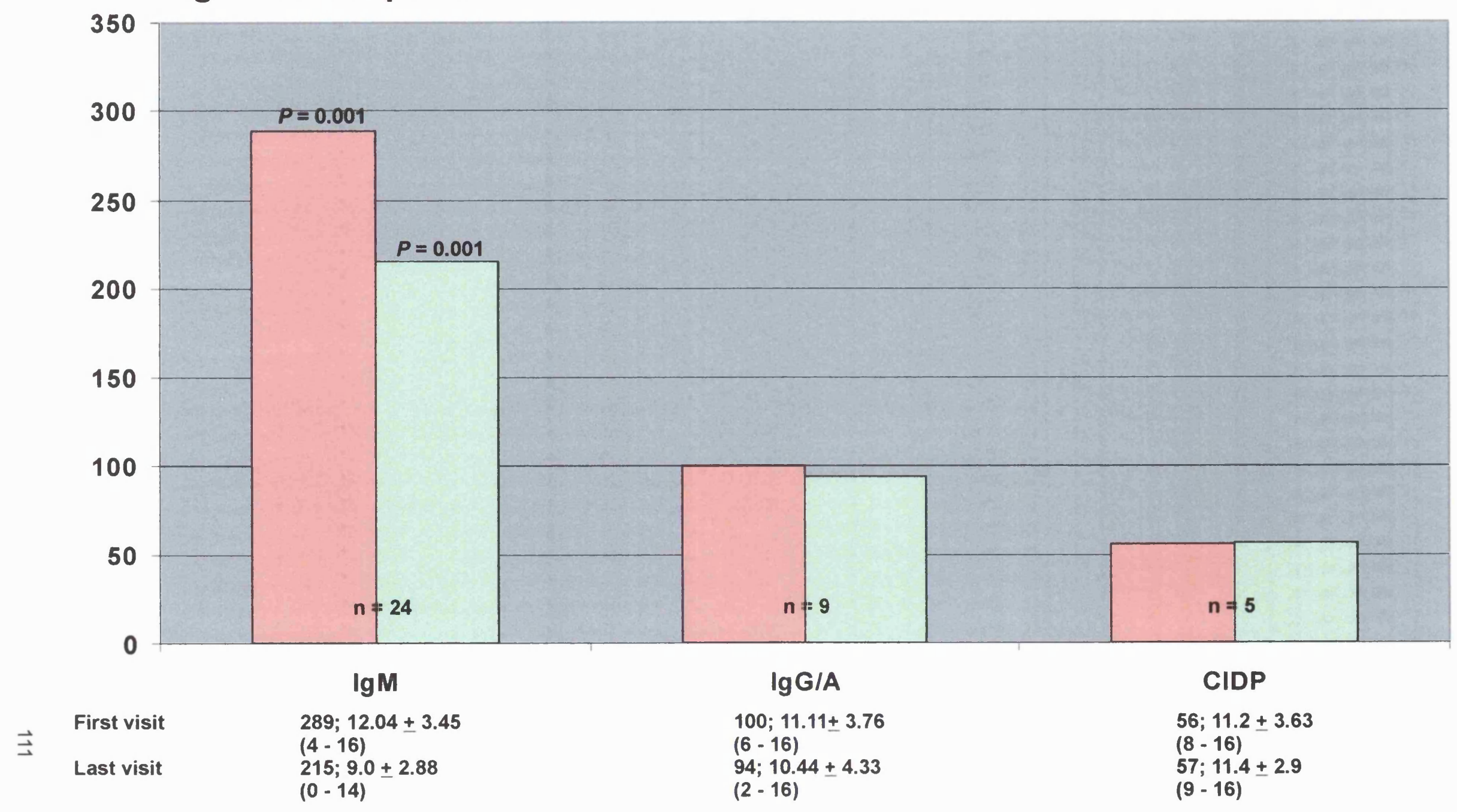


Fig 3.2.6 Vibration sense

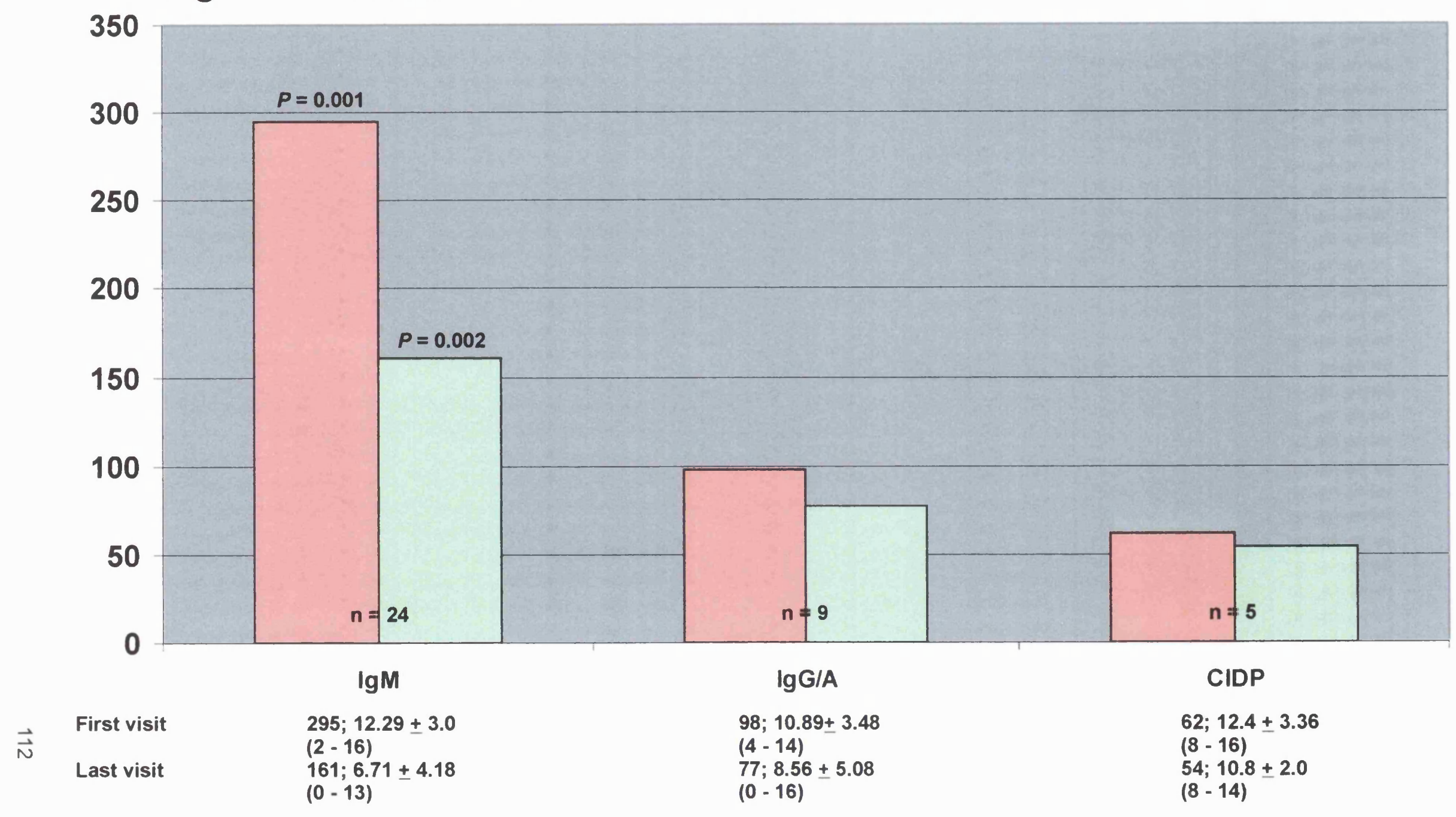


Fig 3.2.7 Joint position sense

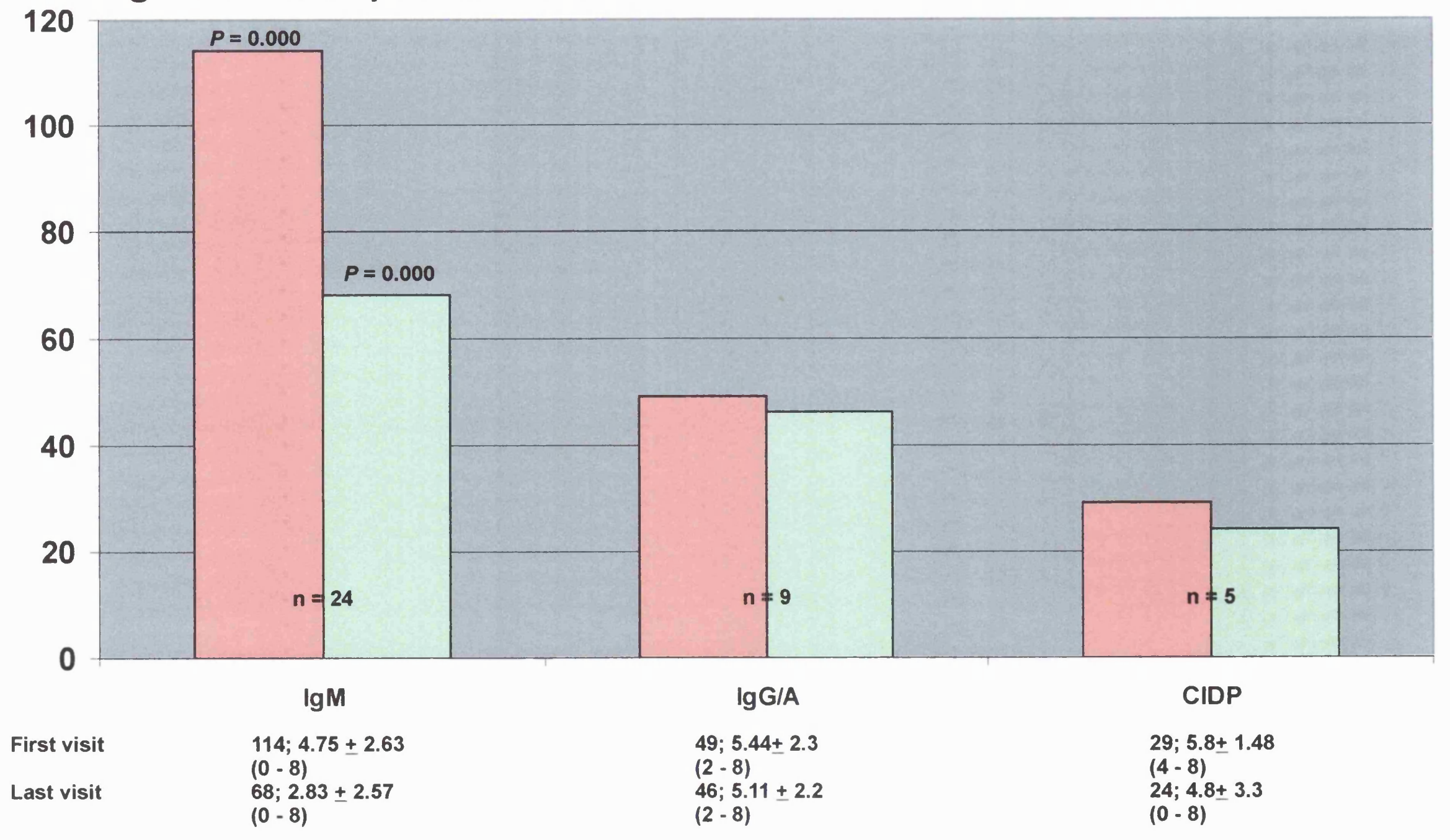


Fig 3.2.8 Disability

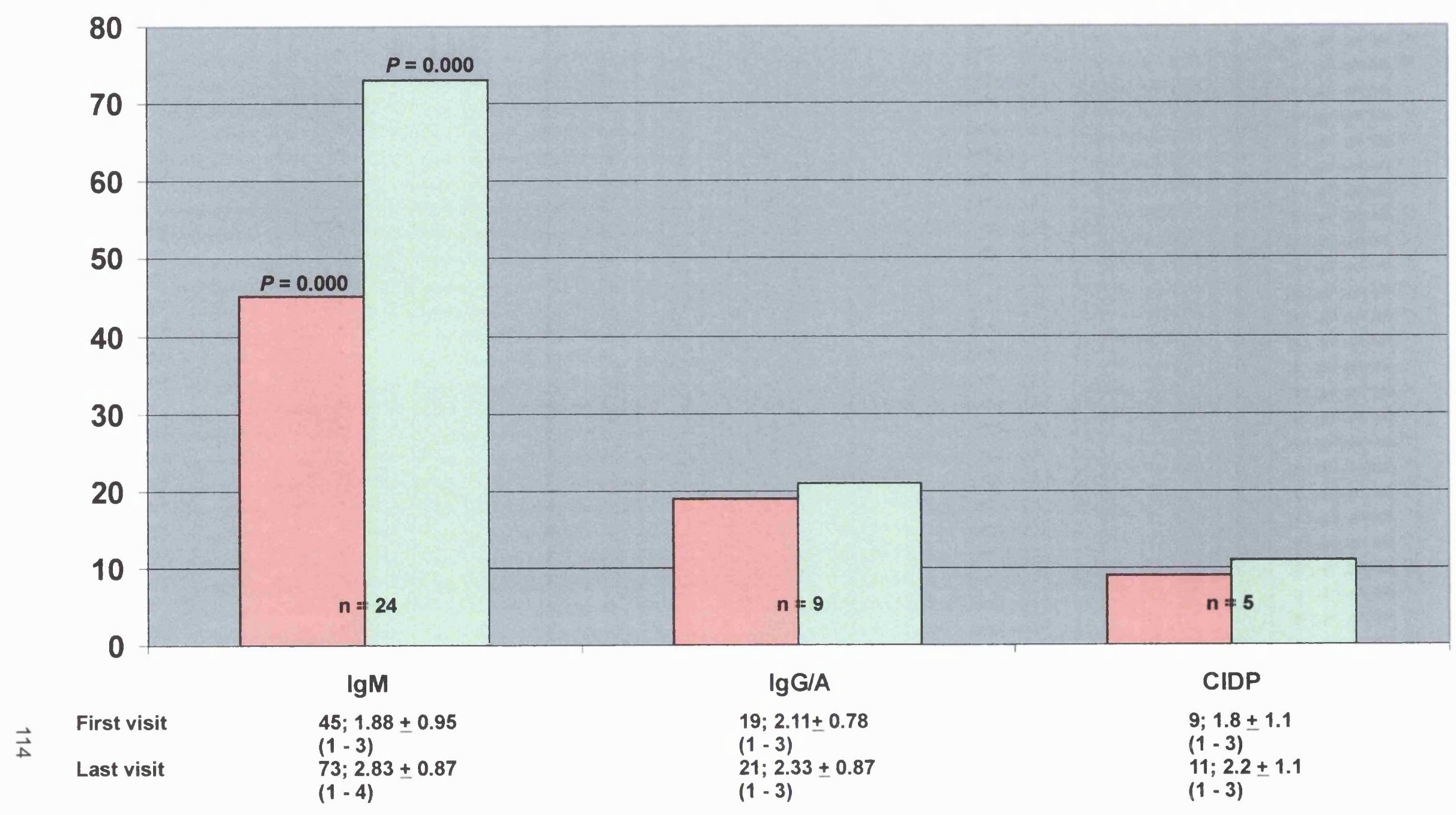


Fig. 3.3

Comparison of scores" between the first and last visits for each IgM subgroup

" summed scores, means \pm S.D. with range in parenthesis.

$P$ : significant difference in values for the same group of patients between the first and last visits. 
Fig 3.3.1 Symptoms

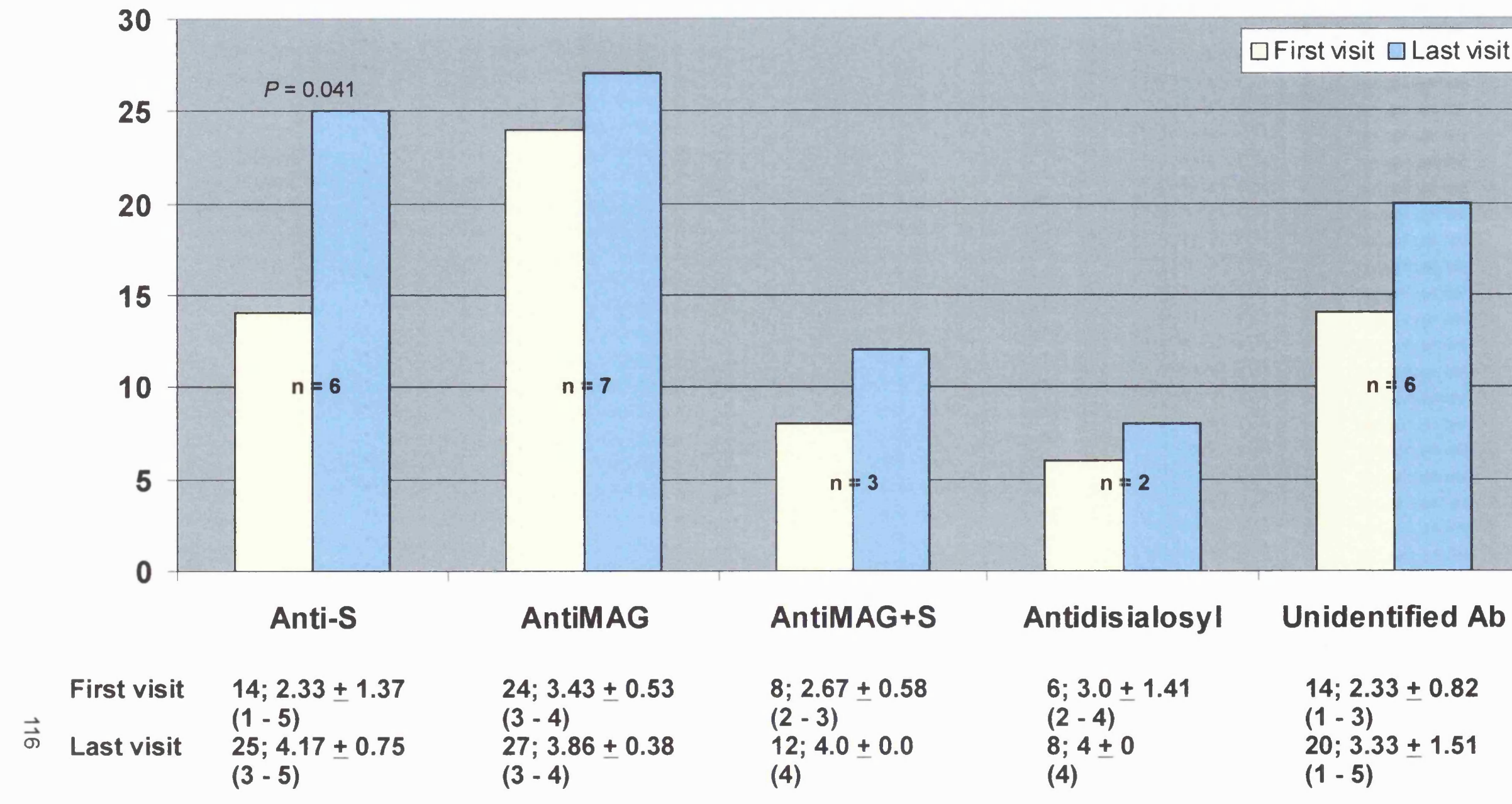


Fig 3.3.2 Muscle strength

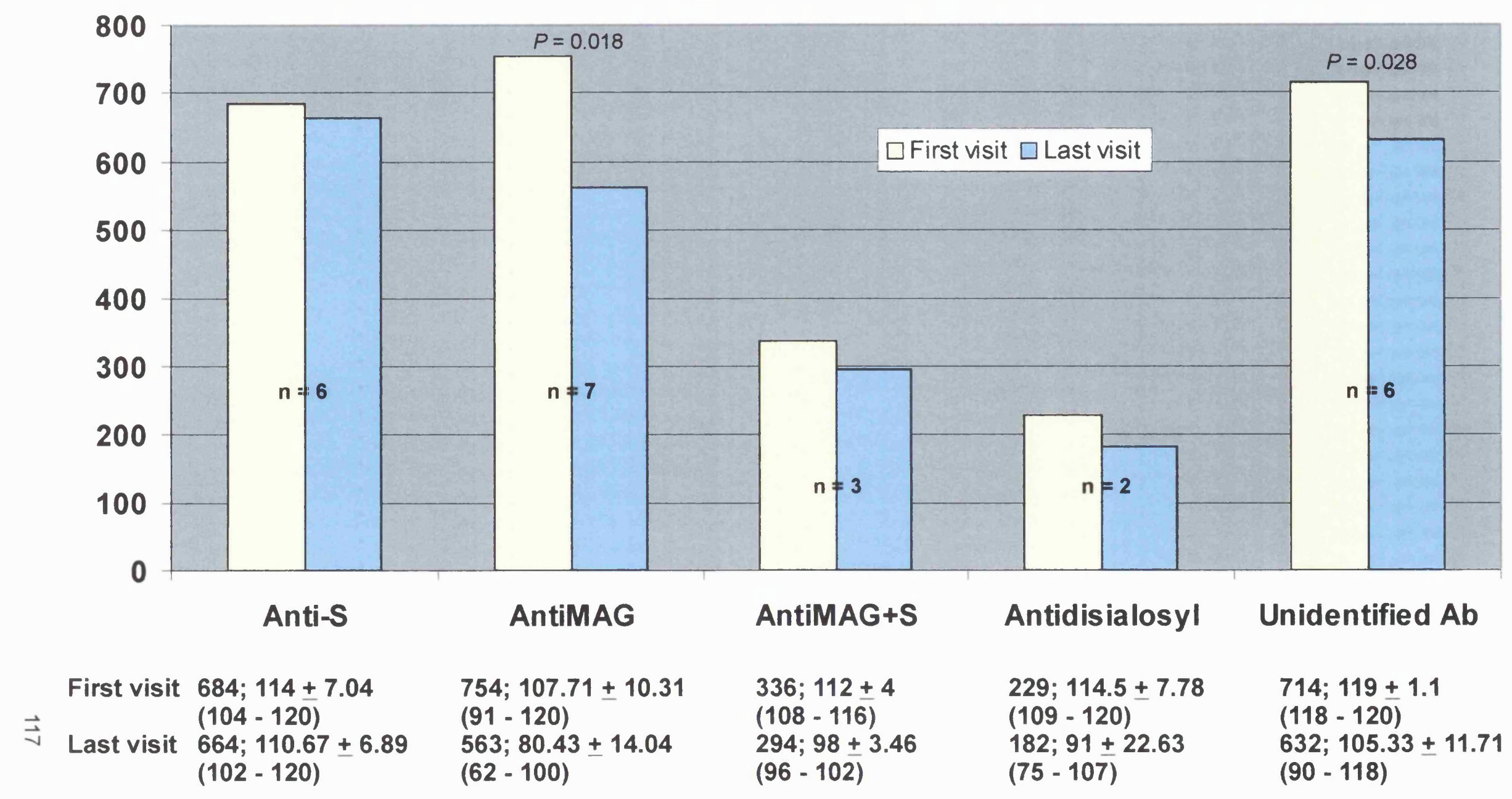


Fig 3.3.3 Tendon reflexes

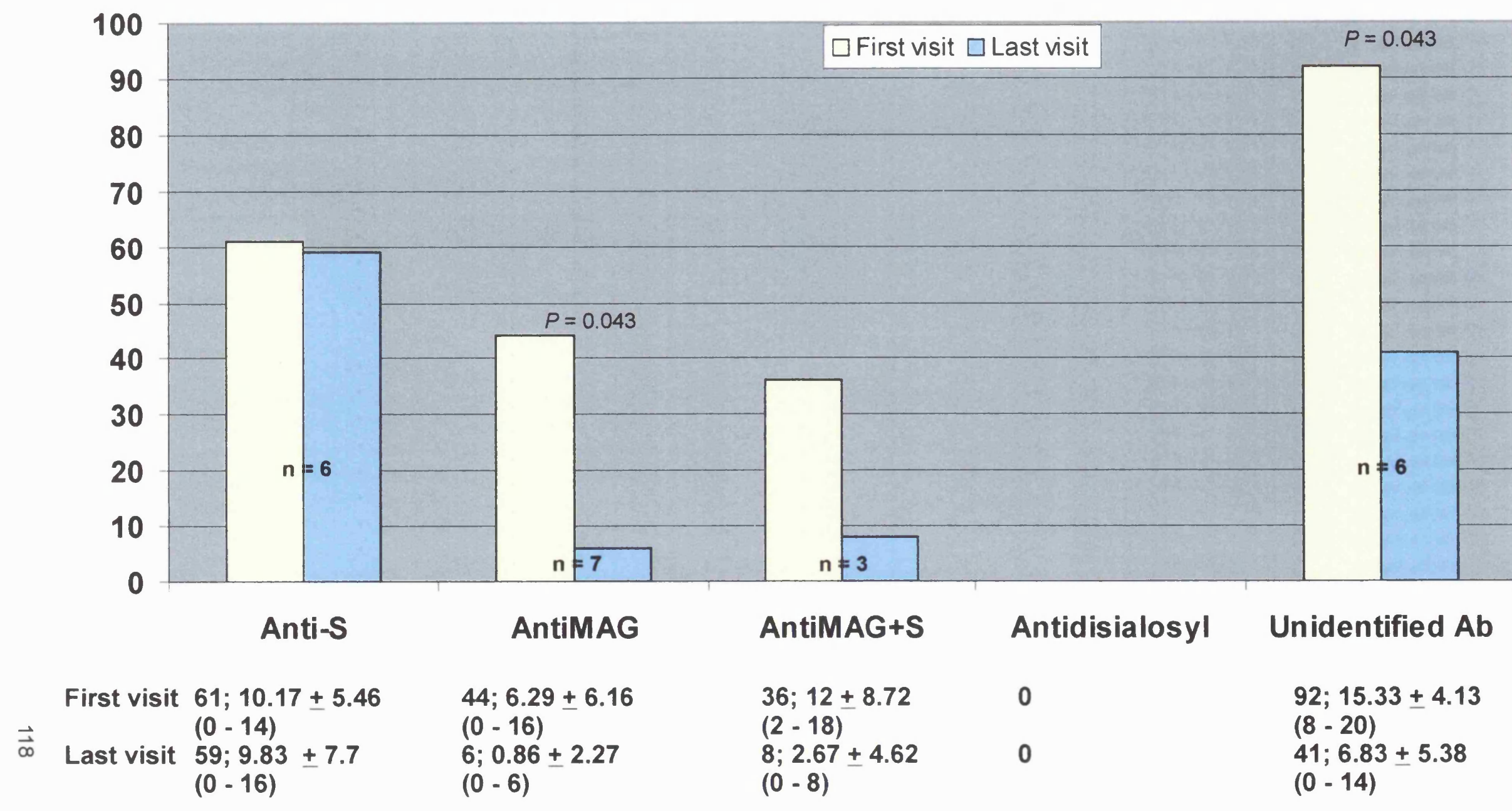




\section{Fig 3.3.4 Light touch}

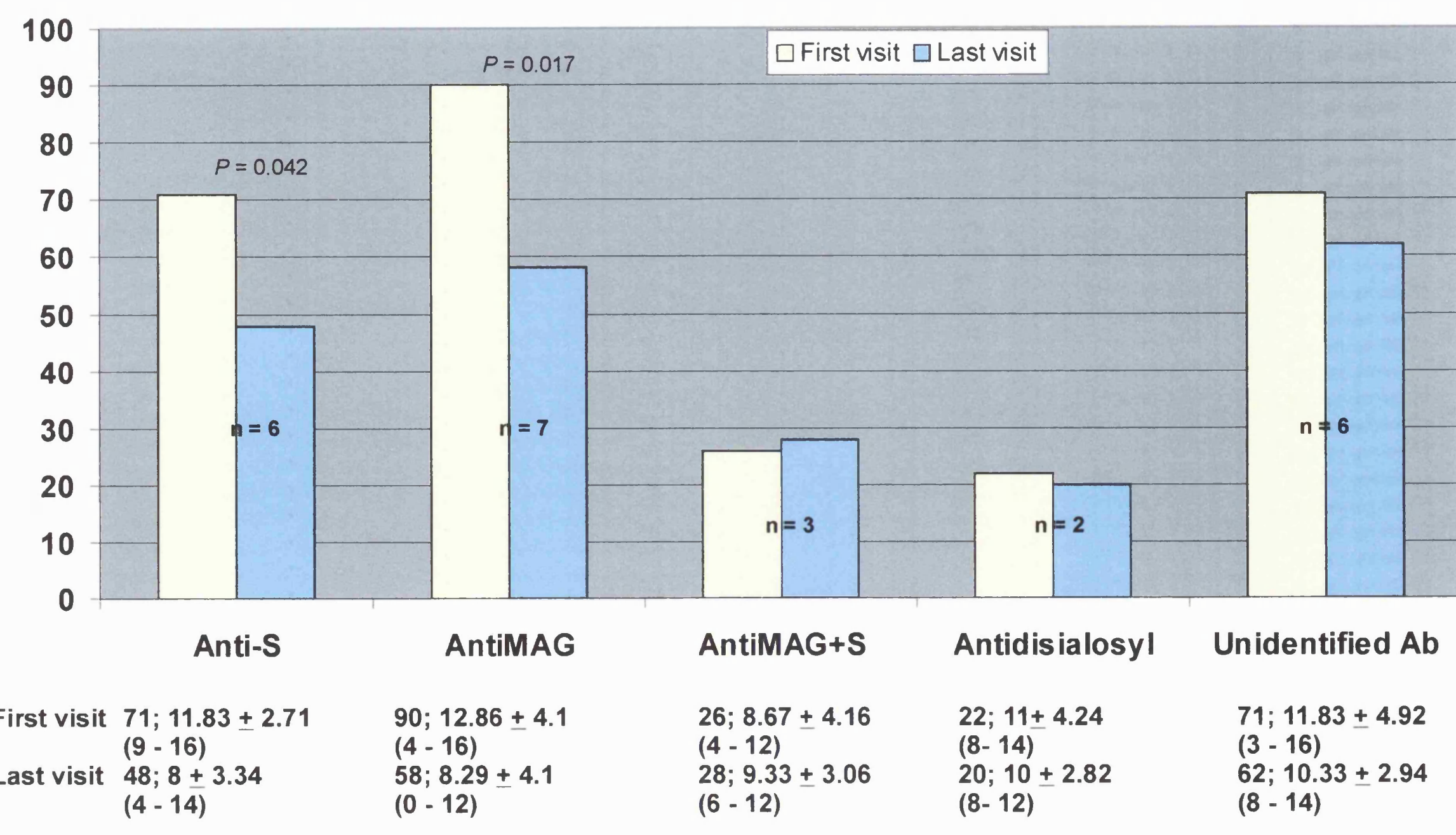




\section{Fig 3.3.5 Pinprick}

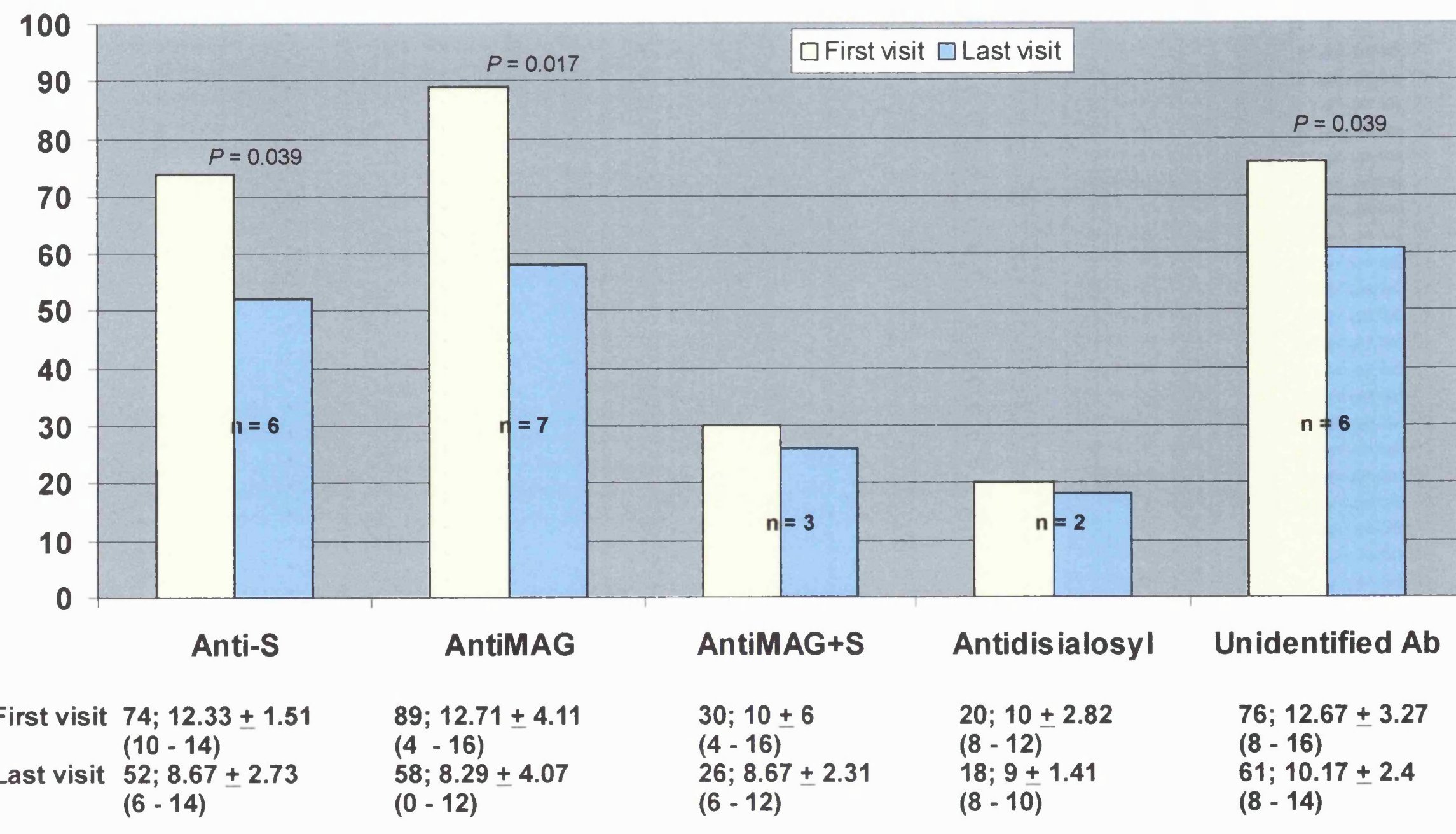




\section{Fig 3.3.6 Vibration sense}

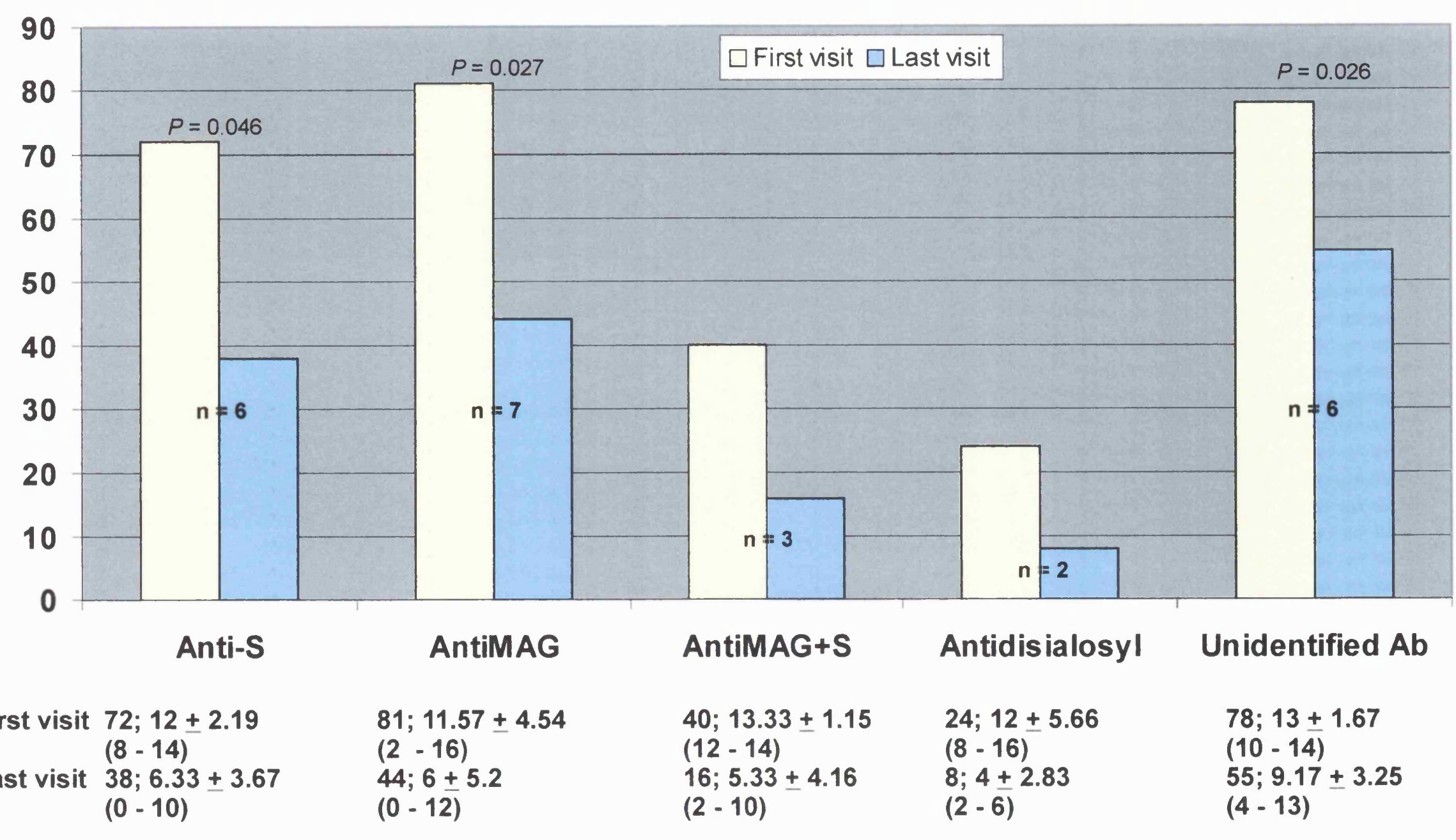


Fig 3.3.7 Joint position sense

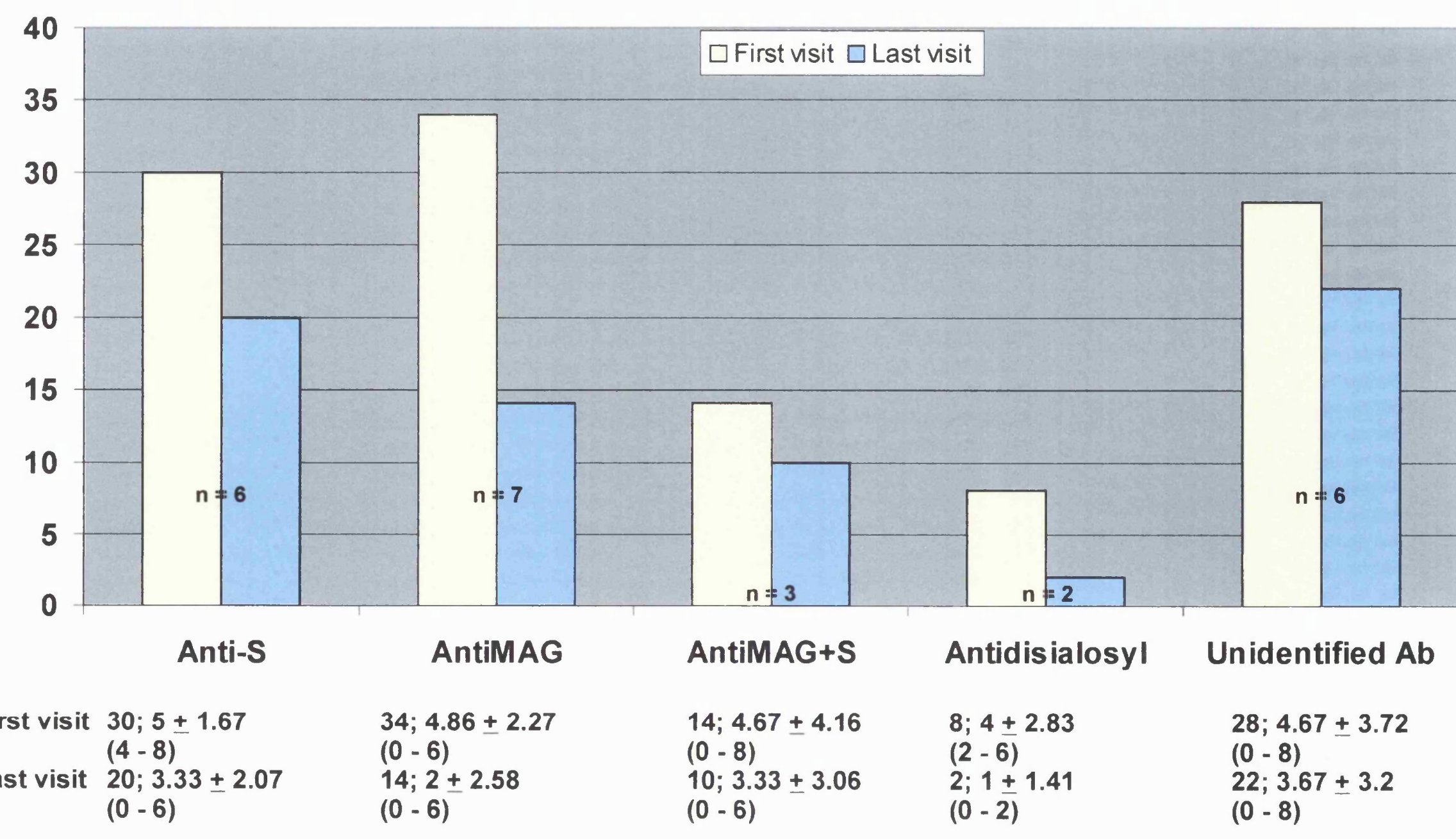




\section{Fig 3.3.8 Disability}

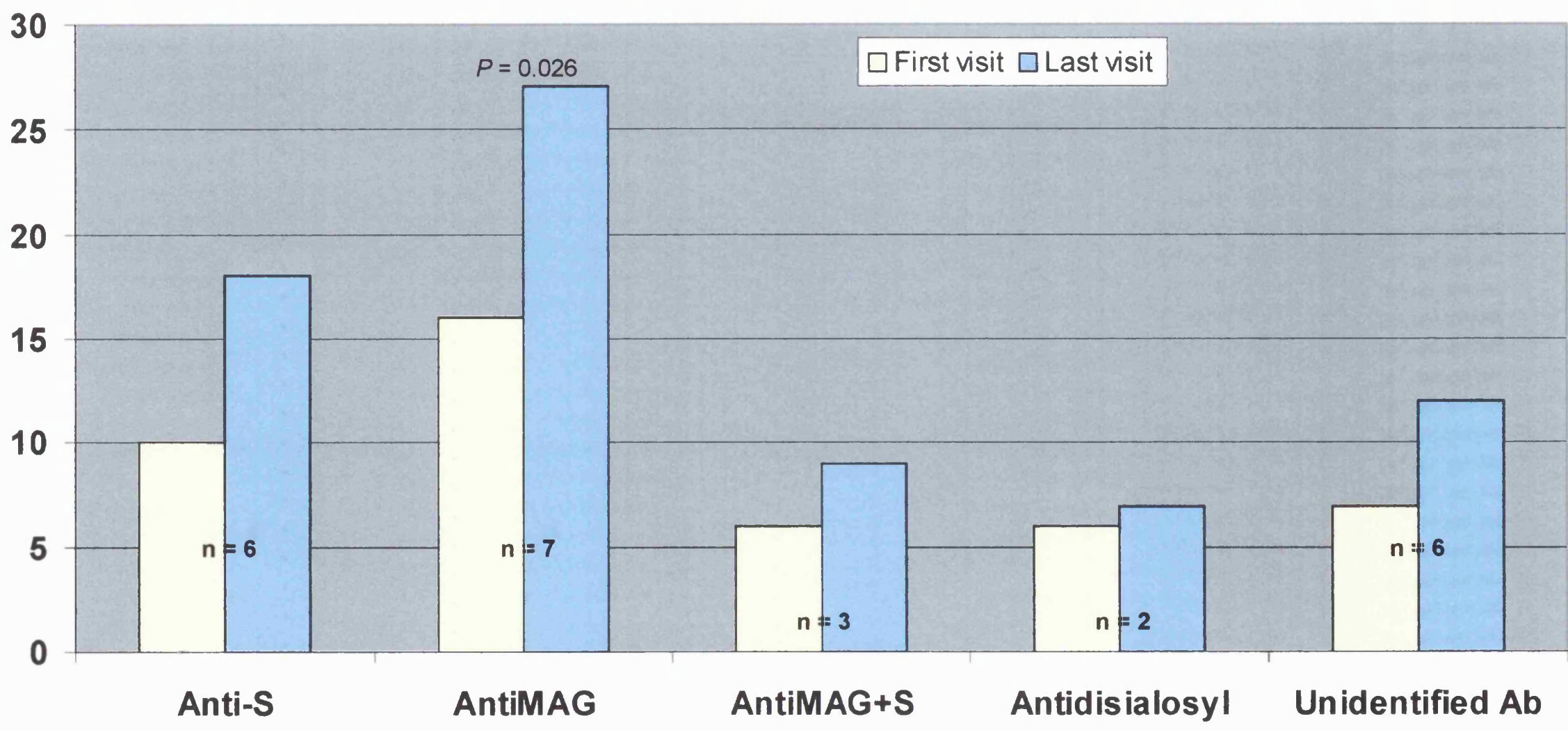

$\begin{array}{llll} & \text { First visit } & 10 ; 1.67 \pm 1.03 & 16 ; 2.29 \pm 0.95 \\ & (1-3) & (1-3) \\ \vec{\omega} & & \text { Last visit } & 18 ; 3 \pm 0.63 \\ & & (2-4) & 27 ; 3.86 \pm 0.38 \\ & & & (3-4)\end{array}$
$6 ; 2+0.95$
$(1-3)$
9; $3 \pm 0$
(3)

$6 ; 3 \pm 0$

(3)

$7 ; 3.5 \pm 0.71$

$(3-4)$

$7 ; 1.17 \pm 0.41$

$(1-2)$

$12 ; 2+1.26$

$(1-4)$ 


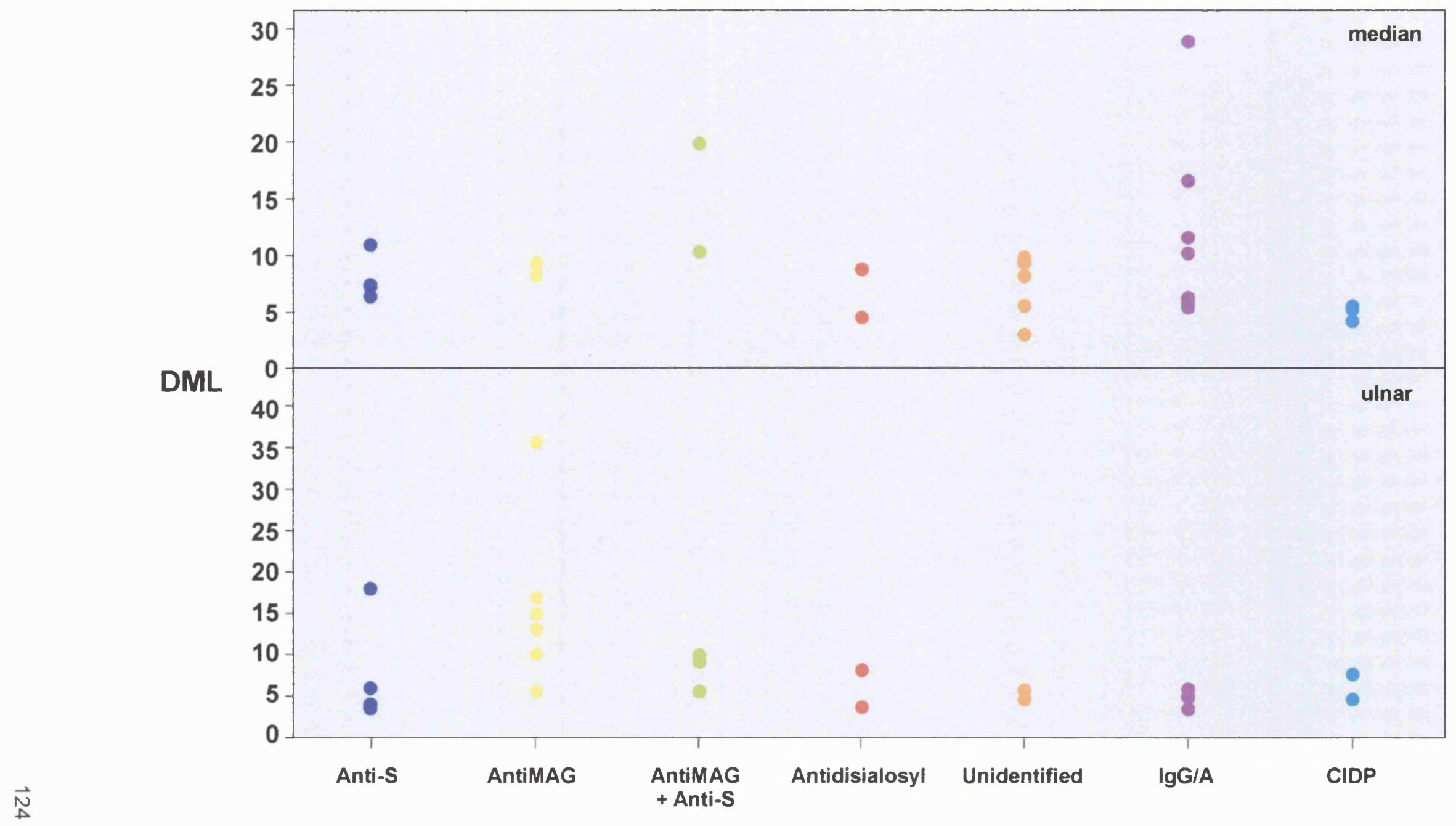

Figure 3.4 Comparison of distal motor latency between various subgroups of IgM, IgG/A paraproteins and CIDP. 


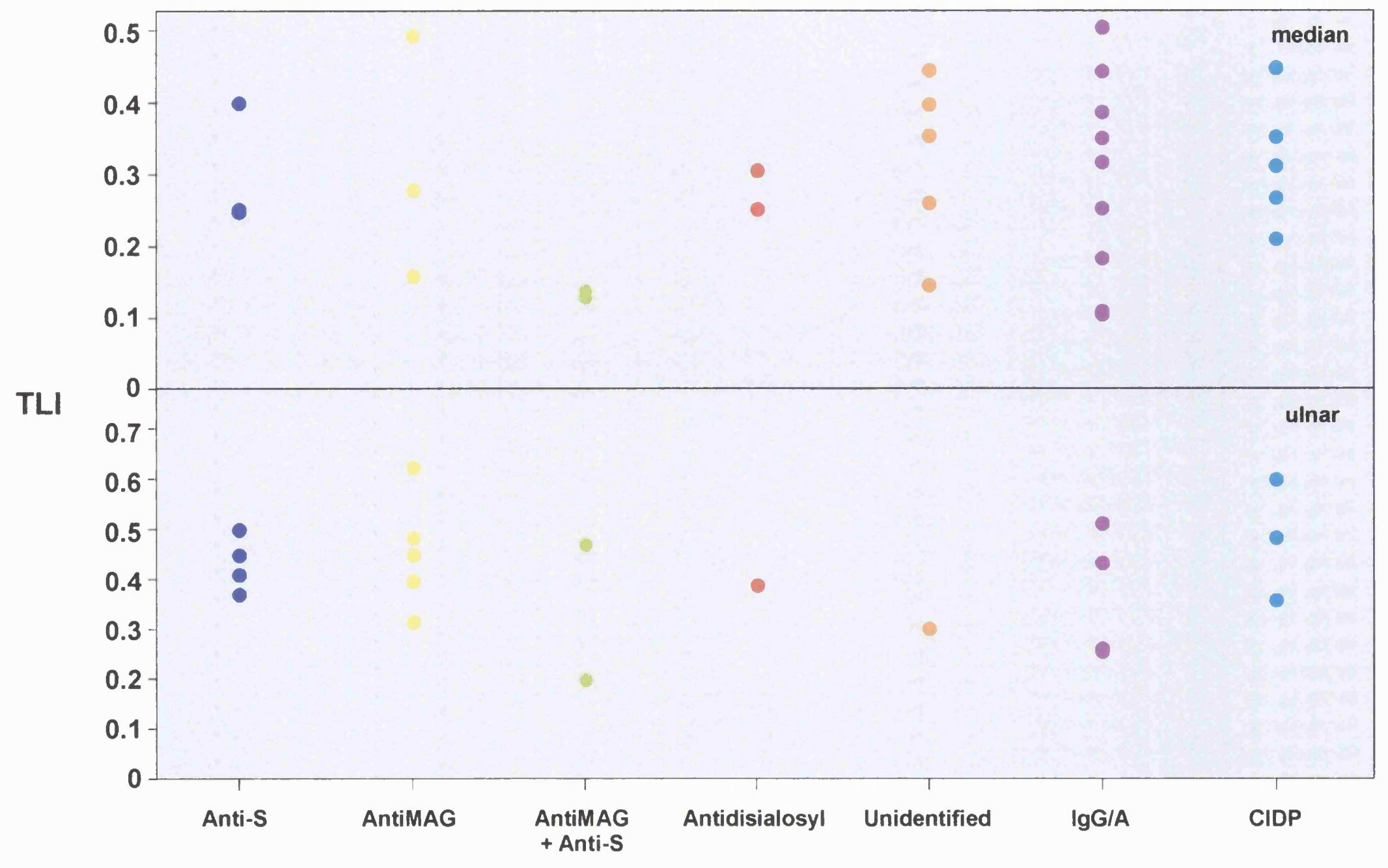

Figure 3.5 Comparison of distal motor latency between various subgroups of IgM, IgG/A paraproteins and CIDP. 

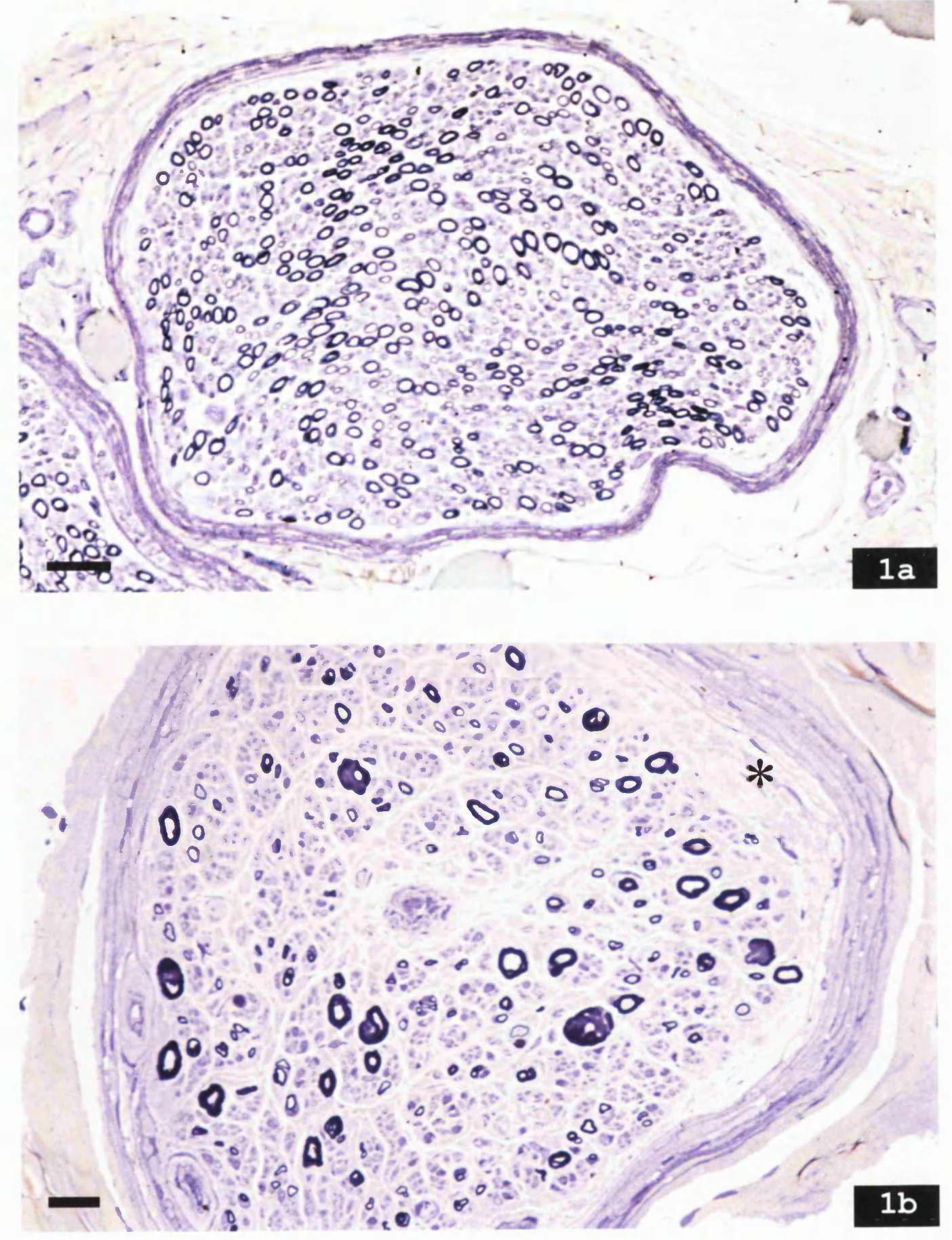

Fig. 3.6.1 Semithin transverse resin sections from sural nerve biopsy specimens stained with thionin and acridine orange.

1a Control subject aged 29 y $\times 270$. Bar $=50 \mu \mathrm{m}$

1b Male aged $67 \mathrm{y}$ with an IgM $\mathrm{k}$ paraproteinaemic neuropathy. There is mild subperineurial oedema (asterisk). Very few large myelinated fibres are seen $x$ 540. Bar $=20 \mu \mathrm{m}$. 

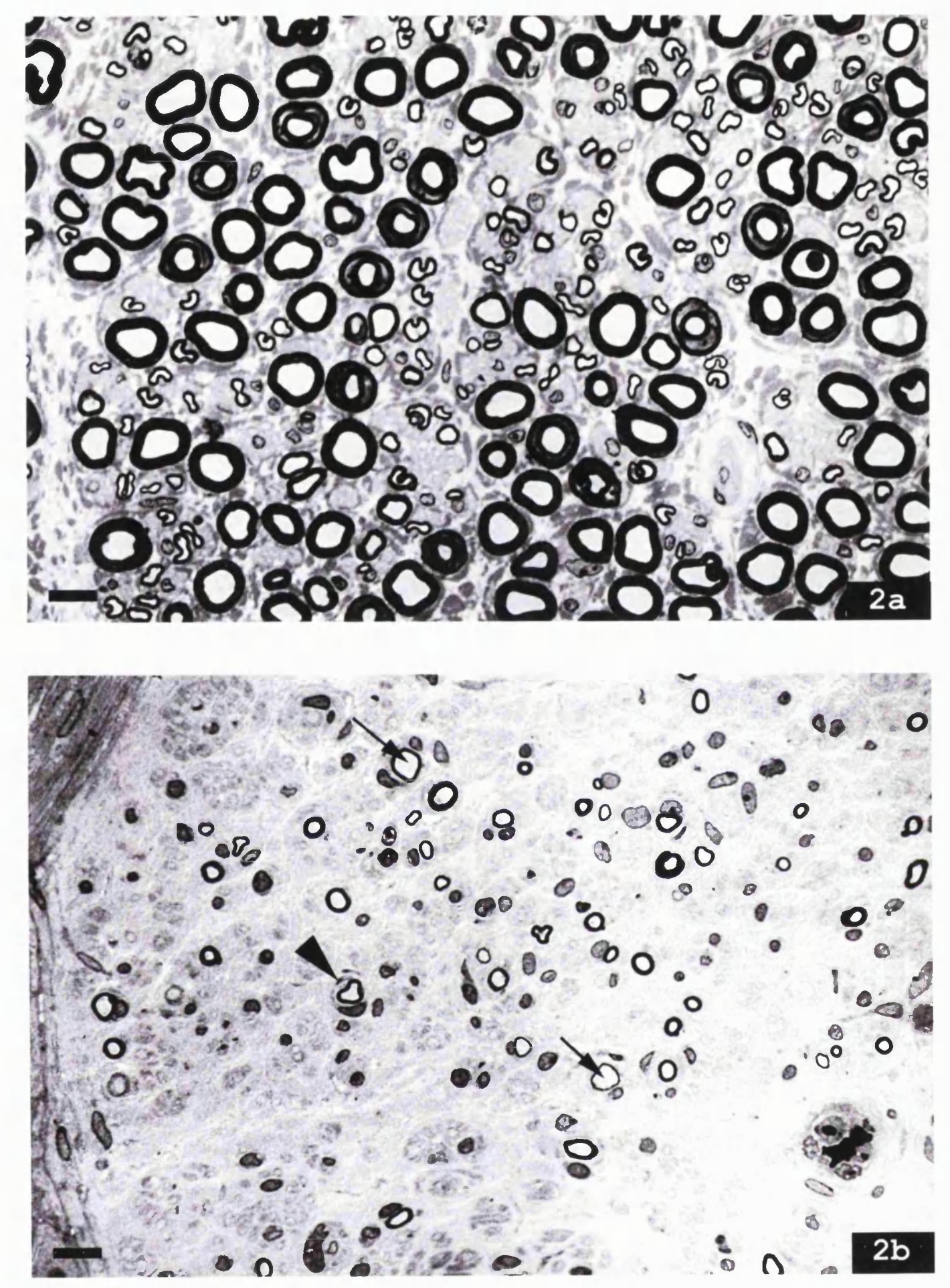

Fig. 3.6.2 Light micrographs of semithin transverse resin sections from sural nerve biopsy specimens $\times 840$. Bar $=10 \mu \mathrm{m}$ for both $a$ and $b$.

2a Control subject aged 65 y

2b Male aged 72 y with an IgMk antiMAG paraproteinaemic neuropathy. Thinly myelinated fibres indicate remyelination (arrows). Arrowhead shows a small onion bulb. No inflammatory cells are seen. The density of myelinated fibres is reduced. 


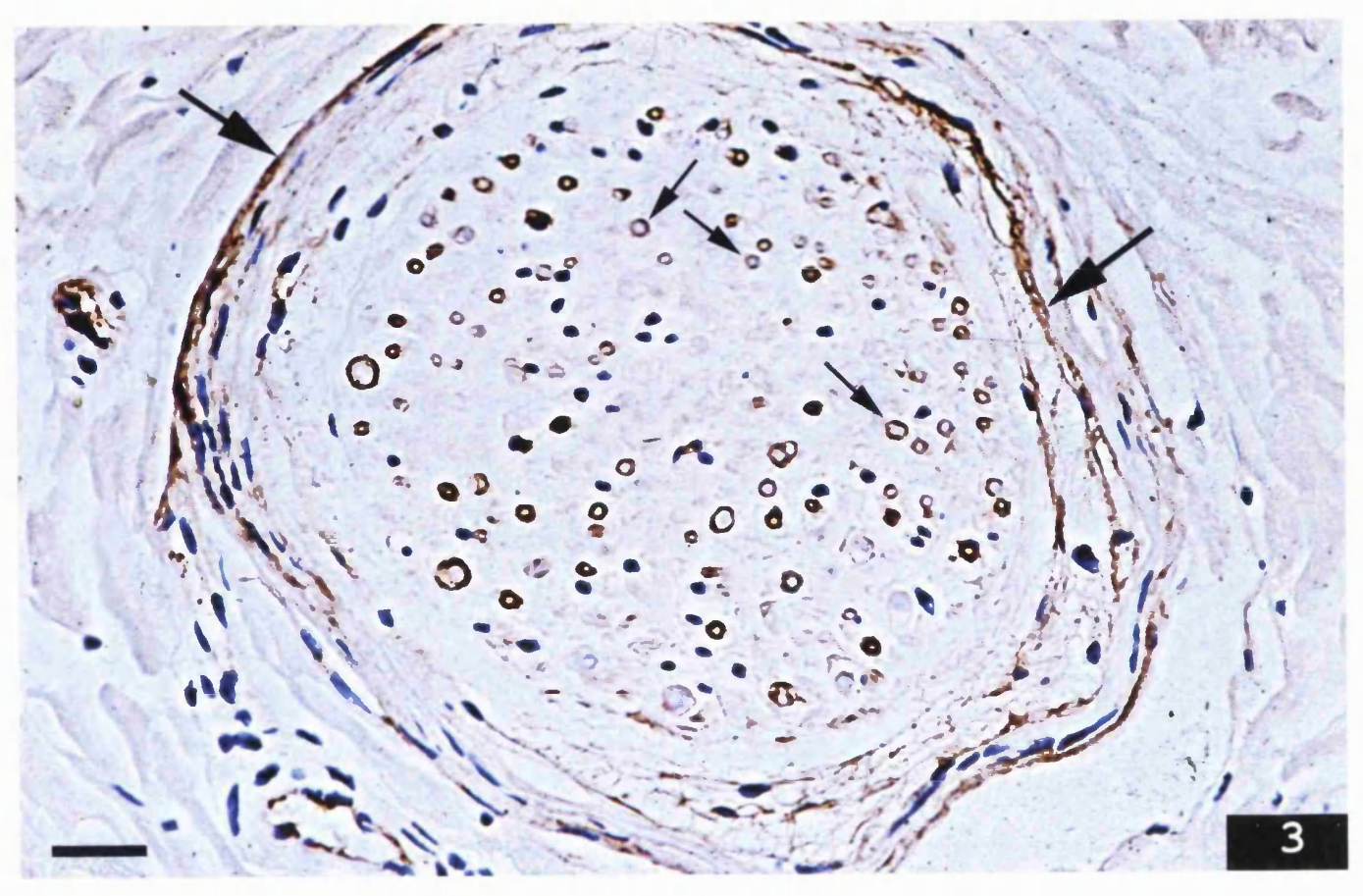

A

B

C

D)

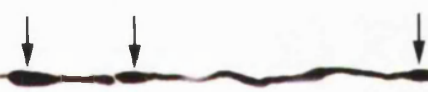

E $\downarrow$ $\downarrow$

$\mathbf{F}$ $\downarrow$ $\downarrow$

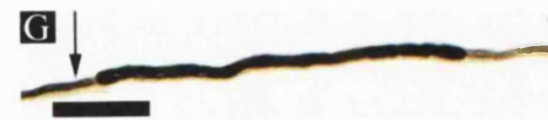

Fig. 3.6.3 Semithin transverse section immunostained to show the presence of lgM (brown staining) from a patient aged $70 \mathrm{y}$ with an IgMk paraproteinaemic neuropathy. A positive reaction is seen on the myelin sheaths of surviving fibres (small arrows) and the perineurium (large arrows) $\times 260$. Bar $=50 \mu \mathrm{m}$.

Fig. 3.6.4 Teased fibres from sural nerve biopsy specimens from patients with benign paraproteinaemic neuropathy. A. Early axonal degeneration showing breakdown of the myelin sheath. B. Later axonal degeneration showing linear myelin droplets. C. Axonal regeneration with regular short internodes. $D, E$. Fibres showing regions of remyelination with thin myelin together with thicker myelin displaying several small tomacula (arrows). F. Intercalated remyelinated segments (arrows), that on the right being thinly myelinated. G. Consecutive segments of paranodal demyelination remyelination (arrows) indicating secondary demyelination. A, C, D, E: IgA paraproteinaemia; B, G: IgG paraproteinaemia; F: IgM paraproteinaemia. A, B, E, F, G: Bar $=50 \mu \mathrm{m} ; \mathrm{C}, \mathrm{D}: \mathrm{Bar}=100 \mu \mathrm{m}$. 

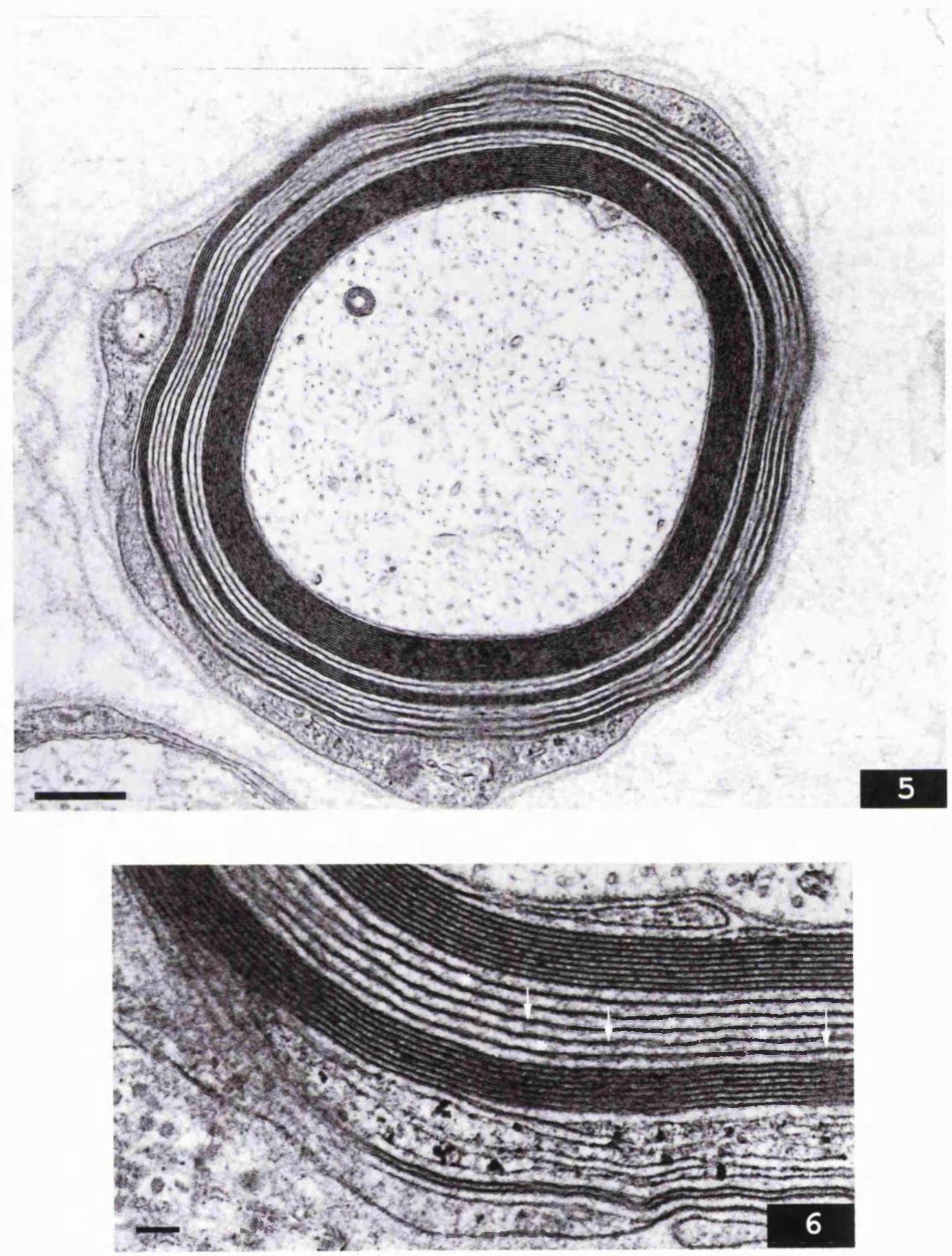

Fig. 3.6.5 Electron micrograph of patient aged 54 y with an IgMk paraproteinaemic neuropathy. Transverse section through an internodal region. The inner myelin lamellae are of normal periodicity contrasting with wide spacing in alternating regions in the outer lamellae. Bar $=0.5$ $\mu \mathrm{m}$.

Fig. 3.6.6 Higher magnification from the same specimen as in Fig. 3.6 .5 showing typical widely spaced myelin in which there is a normal formation of the major dense line (arrows) but the intraperiod line is markedly widened (asterisks). Bar $=0.1 \mu \mathrm{m}$. 

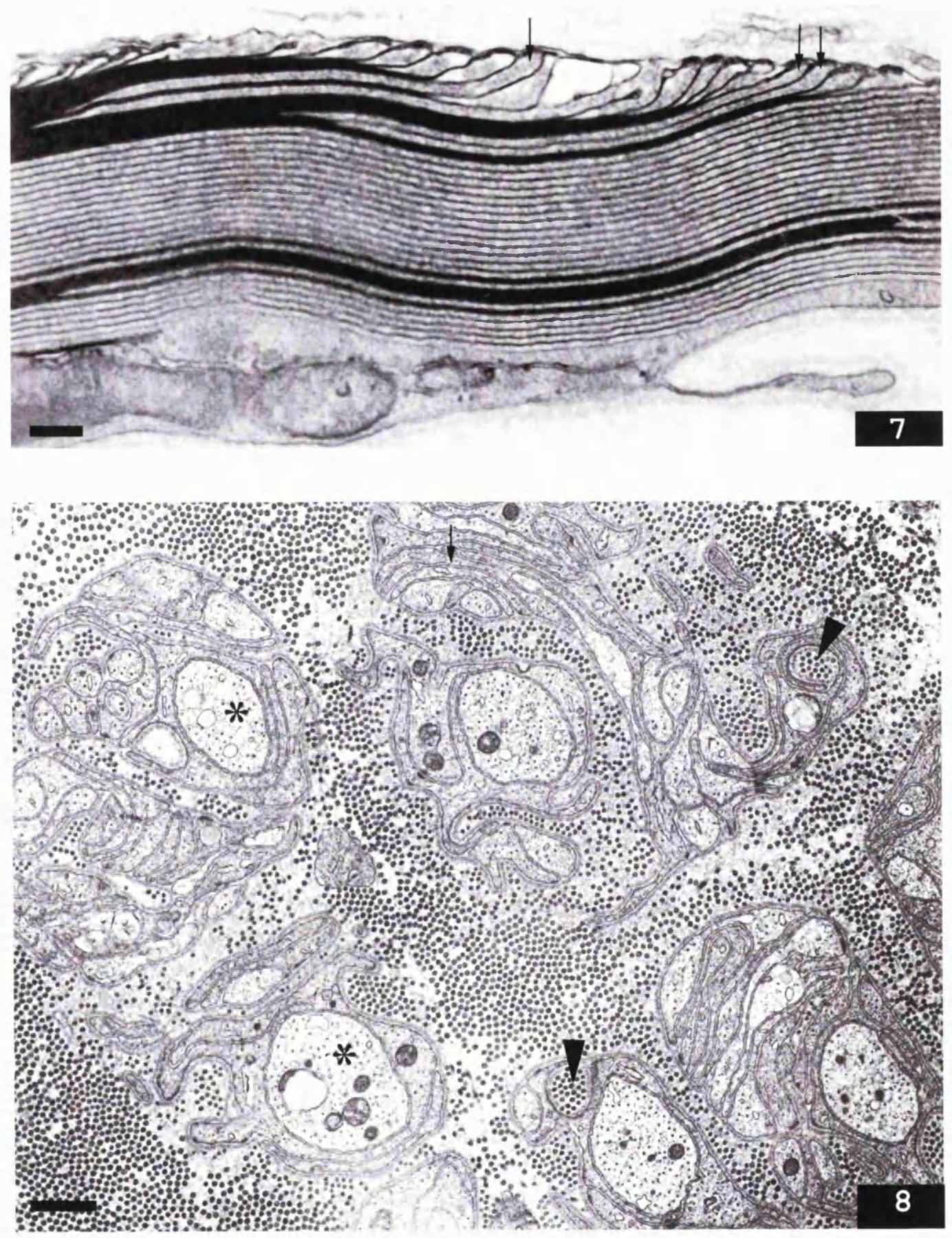

Fig. 3.6.7 Electron micrograph of longitudinal section at paranodal region from a patient aged $60 \mathrm{y}$ with an IgM paraproteinaemic neuropathy showing alternating zones of widely spaced myelin, some of which continue into the terminal loops (arrows). Bar $=0.2 \mu \mathrm{m}$.

Fig. 3.6.8 Electron micrograph of a patient aged 72 y with IgM paraproteinaemic neuropathy showing unmyelinated fibres. Unmyelinated axons are indicated by asterisks. Flattened Schwann cell processes of the type related to loss of unmyelinated axons are seen (arrow). 'Collagen pockets' (arrowheads) are also present. Bar $=0.5 \mu \mathrm{m}$. 


\section{CHAPTER 4}

\section{DISCUSSION}

4.1 Summary of the findings

Demographic data

Incidence of malignancy

Clinical features

Summed scores

Neurophysiological findings

Other laboratory findings

Neuropathological findings

\subsection{Discussion}




\section{DISCUSSION}

\subsection{Summary of the findings}

\section{Demographic data}

Most of the patients (74.5\%) with peripheral neuropathy associated with benign paraproteinaemia had IgM paraproteins predominantly with a $\kappa$ light chain (64\%) and high male:female ratio (18:7). IgA paraprotein was rare. The onset of symptoms was usually in the latter part of the fifth decade and significantly earlier in the CIDP group (mean $36.5 \mathrm{y}$ ). The duration of the follow-up period from the onset of the neurological symptoms was 5-35 y (mean 13.9 y) for patients with benign paraproteinaemia and 7-41 y (mean $17.6 \mathrm{y}$ ) for patients with CIDP.

Amongst the subgroups of patients with IgM paraproteins, $41.5 \%$ were antiMAG \pm antiS positive, $8.3 \%$ had antidisialosyl antibodies and $25 \%$ had antiS or unidentified antibody activity. The pattern of high M:F and $\kappa: \lambda$ light chain ratio was seen only in the antiMAG \pm antiS subgroups. The age of onset of symptoms was significantly earlier in the antidisialosyl group and was not dissimilar to the CIDP group (mean $34.5 \mathrm{y}$ ) although the numbers were small. The interval between the first and the final examination was comparable for the $\lg M$ and $\lg G / A$ subgroups and between the IgM subgroups at approximately $13 \mathrm{y}$.

\section{Incidence of malignancy}

Only $5.5 \%$ of patients developed haematological malignancies. These consisted of Waldenström macroglobulinaemia from B cell lymphoma, red cell anaemia with excess blasts and acute myeloid leukaemia. The interval from the detection of the paraproteins to the diagnosis varied from 11 to $25 \mathrm{y}$. All these patients had been on immunosuppressive therapy for at least 2 y prior to the diagnosis of malignancy.

Nearly $11 \%(10.9 \%)$ of patients had other malignancies of which $66.7 \%$ were of the gastrointestinal tract in location. Two of the patients with gastrointestinal malignancy were still alive after surgical resection 1 
and $4 \mathrm{y}$ ago and had never received treatment for the neuropathy. Other malignancies included carcinoma of the pharynx and metastasis to the liver from an unknown primary.

The paraproteins were $\operatorname{lgM}$ in $88.9 \%$ with a $\mathrm{k}$ light chain in $50 \%$. Kaplan Meier log rank testing failed to show any significant difference in survival at a $90 \%$ confidence level for any type of paraprotein or light chain in relation to death from malignancy.

\section{Clinical features}

All patients presented with distal sensory symptoms progressing proximally. Only those with IgG/A paraproteins and CIDP had sensory symptoms confined to the hands. Paraesthesiae involving the face occurred in the $\operatorname{IgG/A}$ and $\lg M$ with antiS activity groups and were particularly common in patients with CIDP (80\%). Prominent dysaesthesia was observed in all groups of patients. Some showed spontaneous resolution after $3-15 \mathrm{y}$.

Although just over $50 \%$ of patients complained of weakness at the initial visit, on examination all patients had at least mild motor involvement. Weakness usually began in the legs apart from patients with IgG/A paraproteins who had more pronounced weakness in the arms. Facial involvement was only evident in the patients with IgG/A paraproteins. Both patients with IgM antidisialosyl activity had diplopia. Tremor was commonly found as a presenting symptom in patients with IgM paraproteinaemia and pseudoathetosis was evident in cases with severe large fibre sensory deficits that usually showed very low maximal MCV $(\leq 10 \mathrm{~m} / \mathrm{s})$. Nerve thickening was found in only $22 \%$.

Progression was slow and progressive in patients with IgM paraproteins apart from the 2 cases with antidisialosyl activity. This was in contrast with $58 \%$ and $60 \%$ of patients with IgG/A and CIDP with a relapsing/remitting course. 


\section{Summed scores}

On comparison of the summed scores, the $\operatorname{lgM}$ group had more deficits and had more disability than $\operatorname{lgG} / \mathrm{A}$ patients both at the first and last examinations. Improvement in muscle strength and tendon reflexes scores was only observed in the IgG/A group. Amongst the IgM subgroups, the antiMAG group showed a significant overall deterioration on follow-up.

\section{Neurophysiology}

Only $8.2 \%$ of patients with IgM paraproteins had an axonal neuropathy. The remainder of the patients had a predominantly demyelinating neuropathy.

Although there was no significant difference between the mean values for the MCV, DML and TLI between the various subgroups of IgM and IgG/A paraproteinaemia and CIDP, the latter two appeared to outperform IgM group in qualitative analysis. Improvement either in sensory recording or $\mathrm{MCV}$ was seen only in the $\lg$ G/A and CIDP groups.

Distal CMAPs were significantly lower and ulnar RLs were significantly prolonged in the antiMAG subgroup at the last examination. There was a significant prolongation of the DML in comparison with the $M C V$ in the forearm in the antiS and antiMAG groups at the first visit which was not evident at the last visit. In the only case with antiMAG activity in which measurement of central conduction time was undertaken by magnetic stimulation, the MCVs were fairly uniform in comparison with the 2 cases with IgG paraproteins.

\section{Other laboratory findings}

Cerebrospinal fluid protein. This was elevated in $>60 \%$ of all cases, particularly in the IgG/A groups (100\%). The maximal level which was $4.8 \mathrm{~g} / \mathrm{l}$ was observed in a patient with $\mathrm{lgG}$ paraproteinaemia. 
Serum paraproteins. These were detected only after repeated testing in 3 cases $(6 \%)$. The paraprotein was not detected in $4 \%$ and $8 \%$ had developed a bi or triclonal gammopathy at the last follow-up.

Other autoantibodies. These were positive in 2 out of 3 cases with IgM anti MAG and anti-S activity.

\section{Neuropathological findings}

The presence of widely spaced myelin and immunoglobulin deposition were only found in patients with IgM paraproteinaemia. Of the 34 patients with paraproteinaemia who had nerve biopsies, in 21 patients the changes were predominantly demyelinating whilst they were mixed axonal and demyelinating in nature in the remainder.

\subsection{Discussion}

The incidence of haematological malignancy on follow-up of patients with benign paraproteinaemia neuropathy is substantially lower than in patients ascertained without peripheral neuropathy. A low incidence at approximately $6 \%$ was noted on follow-up studies with a shorter interval of 5 y (Ellie et al., 1996; Notermans et al., 1994; Smith, 1994) as well as in the present study at $5.5 \%$ at a considerably longer interval (up to 42 y). Most of the patients had an IgM paraproteinaemia. In 1 patient, the malignancy was B cell related. None developed multiple myeloma. This is in contrast to the $68 \%$ incidence amongst the "plasma cell dyscrasia" group of patients with benign monoclonal gammopathy reported by Kyle (1995). A review confined to patients with benign IgM monoclonal gammopathy, by the same author in the same article, failed to show any patient with multiple myeloma during a 22 y follow-up. The malignant lymphoproliferative disorders were not dissimilar and included chronic lymphocytic leukaemia, Waldenström macroglobulinaemia and lymphoma. As malignant IgG/A monoclonal gammopathies are observed to be associated with multiple myeloma (Kyle et al., 1969; Linos et al., 1981), the discrepancy in the incidence 
of multiple myeloma may therefore be related to the high representation of IgG $(73 \%)$ when considering patients with monoclonal gammopathy of undetermined significance as a whole. The low incidence of haematological malignancy, the high incidence of IgM paraproteins in patients with peripheral neuropathy associated with benign monoclonal gammopathy and the lack of increasing incidence with time suggest a different pathogenetic mechanism(s) from those without neuropathy. As the resulting malignancies are similar for the same $\mathrm{lg}$, a postulation has to be made that whatever the mechanisms are, they share the common pathway: plasma cells or their B cell precursors.

The earliest identifiable member of the $B$ lymphocyte lineage is the pre-B cell. Within the pre-B cells, a series of genetic translocations and possibly somatic changes occur involving the gene codes for both the heavy $(\mathrm{H})$ and light $(\mathrm{L})$ chains. Completion of the process, with the help of interleukins 7,6 and 4, is associated with the appearance of Ig on the surface of the cells resulting in B cells. Immature B cells only express membrane $\lg$ of $\lg \mathrm{M}$ class whilst mature $B$ cells express both membrane IgM and IgD. Resting $B$ cells enter into DNA synthesis stimulated by interleukin 4, proliferate with interleukin 5 and differentiate into plasma cell with interleukin 6 . Elevated levels of interleukin 6 have been found in patients with multiple myeloma. It has been postulated that expression of interleukin 6 induces a polyclonal proliferation of plasma cells and that a second event such as altered oncogene expression may transform the cells into a monoclonal process (Kyle, 1988). As the levels of interleukin 6 are not elevated in patients with benign monoclonal gammopathy, the induction of the proliferation of a single clone may occur at any level from pre-B to mature but resting $B$ cells and may be the result of the combination of several mechanisms. Whilst the underlying process remains unknown, precipitation by the administration of cytotoxic drugs has to be considered. Immunosuppressive treatment of patients with benign IgM with antiMAG neuropathy should be circumspect in view of the lack of established 
response to treatment, the side effects and the possible risk of malignancy.

Haematological malignancies usually occur more than 10 y after the initial detection of the paraprotein (Giraldo et al., 1991; Kyle, 1995). The malignancies may develop acutely or progressively and are not necessarily preceded by a sudden rise in the paraprotein concentration. Some are heralded only by a sudden deterioration in the neuropathy with stable paraprotein levels and other laboratory results (Notermans et al., 1994). Patients with IgA gammopathy were found to have a high probability of malignant transformation (Blade et al, 1992). Isaksson et al (1996) reported a progressive increase in the presence of blood clonal $B$ cell excess (as defined by an increase in lymphocyte $k / \lambda$ light chain ratio $>3.5$ for $\kappa$ and $>0.9$ for $\lambda$ light chains) prior to the diagnosis of malignancy in benign paraproteinaemia and proposed blood clonal $B$ cell excess as a powerful indicator for malignant transformation. However, only $53 \%$ of patients with excess values developed malignancy and both studies were performed only in patients with benign gammopathy without neuropathy. At present there appear to be no single sensitive and reliable predictor for malignant haematological transformation for benign paraproteinaemic patients with or without peripheral neuropathy. Regular clinical and routine serum paraprotein level follow-up of these patients should be carried out for an indefinite period of time.

Nonhaematological malignancy especially of the gastrointestinal system constitutes a common cause of death especially in patients with benign paraproteinaemic neuropathy (Smith, 1994 and present results), but may reflect the high incidence of colorectal and pancreatic malignancy in a population of this age. Nevertheless, the possibility of induction of nonhaematological malignancy by treatment with cytotoxic drugs cannot be dismissed. 
IgM paraproteinaemic neuropathy, as a whole, consists of a number of heterogeneous subgroups. Benign monoclonal IgM paraproteinaemic neuropathy with antiMAG activity, which constitutes the largest subgroup of the IgM paraproteins, appears to form a uniform clinical and neurophysiological entity. The incidence of patients with antiMAG activity is lower at $42 \%$ in comparison with the $50-60 \%$ found in other series (Latov, 1995; Quarles, Weiss, 1999; Ropper, Gorson, 1998). The explanation for this is uncertain. The present study confirms the late onset, male predominance and an initial distal symmetrical sensorimotor neuropathy. At first the large sensory fibres are predominantly involved in particular vibration sense. Postural tremor which usually develops after 1.5 to 12 y (mean 4.7 y) (Smith, 1995 ) is often seen in the early stage of the disease for the patients with benign IgM paraproteins. An early onset of tremor has previously been observed by Bain et al (1996). Upper limb postural tremor is frequently evident but significant tremor in the legs is rare (Provinciali et al., 1989; Spencer, Moench, 1980). Tremor is usually postural or action in type and occasionally occurs at rest, predominantly affecting the distal muscles and is often irregular and variable in amplitude (Smith et al., 1983; Smith et al., 1984). The presence of tremor in the face of normal joint position sense, the correlation of tremor frequency in the thumb and ulnar conduction velocities (Smith et al., 1984) and the absence of any demonstrable lesion in the central nervous system suggest that the tremor is due to a defect in the peripheral sensory input and independent of joint position sense. As tremor is rare in the legs, the mechanism involves the distortion rather the absence of sensory input. Following several neurophysiological investigations including ballistic wrist movements, Bain et al (1996) concluded that the tremor occurs as a result of mistimed, distorted peripheral inputs which result in a functional impairment in the cerebellum and the other related circuits producing the delay in second agonist bursts. The hypothesis is supported by positron emission tomography activation studies which 
have shown hyperactivity of the cerebellar hemispheres bilaterally both at rest and during the occurrence of tremor (Brooks et al., 1992; Colebatch et al., 1990; Jenkins et al., 1993). Imaging of the cerebellum has been consistently normal. Progression of the neuropathy has been slow but steady in all patients in the present series resulting in significant disability from motor as well as sensory deficits and in some cases disabling tremor.

Nerve conduction studies in patients with IgM antiMAG paraproteinaemic neuropathy show a demyelinating neuropathy in all cases. Motor conduction velocity in the more proximal segments is fairly uniform. Conduction block is rare. The finding of stable maximal motor conduction velocities after 5 y by Smith (1994) is confirmed in the present study but serial clinical and neurophysiological findings suggest a progressive axonal degeneration extending more proximally. Kaku et al (1994) drew attention to a relative prolongation of distal motor latency in patients with IgM paraproteinaemic neuropathy with antiMAG antibodies and antibodies to sulphated glucuronyl paragloboside, indicating a greater prominence of demyelination peripherally. Similar findings were observed at the time of the initial electrophysiological studies in the present series but were no longer evident after prolonged follow-up times. This suggests a centripetal progression of the demyelination or secondary axonal degeneration leading to normalisation of the TLI from loss of severely affected fibres. The latter explanation is supported by the significantly reduced mean distal CMAPs in the antiMAG group. It is of interest to note that the distal CMAPs are relatively preserved in patients with IgM paraproteins with antiMAG and anti-S activities. This finding needs to be confirmed in a larger group of patients before a definite conclusion can be drawn. The constant extensive segmental demyelination with hypertrophic changes contributes to the substantial slowing of nerve conduction although some of the slowing may be related to conduction in regenerating fibres. 
It is generally accepted that peripheral neuropathy associated with IgM antiMAG activity is an autoimmune neuropathy and that the reactive epitope in MAG is the carbohydrate part of the molecule shared with a number of other neural glycoconjugates including the Po and PMP 22 myelin proteins as well as with SGPG and related glycolipids. Whilst MAG and Po are restricted to the uncompacted myelin and compacted myelin respectively, PMP22 is mainly localised in compact myelin and is also present in the Schwann cell surface membrane both in myelinated fibres and Remak fibres. As the reactive epitopes are mainly located in the myelin, it is not surprising that the neuropathy is demyelinating.

The reason for the axonal degeneration is, however, unclear. This is particularly puzzling when the axonal degeneration is progressive even when the MCV, which reflects the degree of demyelination, is relatively stable. It seems unlikely that the secondary axonal degeneration that often accompanies demyelination is the sole explanation for the progressive change. As SGPG has a wider distribution than the glycoproteins of myelin sheaths, being present in Schwann cell and neuronal membranes, it seems plausible that the IgM antiMAG activity which is also known to display reactivity to the L2/HNK-1 epitopes on SGPG (or other glycoproteins and glycolipids) might be responsible for the axonal change. Chassande et al (1998) reported that reactivity with MAG was associated exclusively with demyelinating neuropathy whereas reactivity with SGPG was found in some axonopathies.

Of considerable interest are the 2 patients with antibody activity against antidisialosyl groups. Both shared a similar clinical phenotype (CANOMAD) consisting of fluctuating oculofacial and/or bulbar symptoms on a background of slowly progressive ataxic large fibre sensorimotor neuropathy. The paraproteins were both $\operatorname{Ig} M \lambda$ which reacted with disialosyl groups on the gangliosides as well as having cold agglutinin activity with Pr2 or Pr1. The symptoms of ataxia, 
ophthalmoplegia and the presence of GQ1b antibodies resemble the Miller Fisher syndrome although the latter is an acute self limiting neurological disorder with polyclonal IgG reacting with GQ1b. The relapsing remitting course, the response to intravenous immunoglobulin therapy and in some cases, the neurophysiological findings make it difficult to distinguish CANOMAD from CIDP. This group of patients therefore forms a distinct clinical and immunological entity. It is important to segregate such patients from the rest of the patients with peripheral neuropathy associated with benign IgM monoclonal gammopathy in view of the possible benefit of treatment and their outcome.

Although the IgM paraproteins in these patients tend to have preference for the disialosyl structure as the dominant reactive determinant, there is a variation in paraprotein affinity to the disialosyl terminals amongst the patients reported in the literature (Arai et al., 1992; Duane et al., 1992; Herron et al., 1994; llyas et al., 1985; Obi et al., 1992; Willison et al., 1993; Yuki et al., 1992). The serological variation may be related to the fine specificity of the antibodies. The precise site of the reaction with the simplest carbohydrate denominator appears to be responsible for the variation in the clinical manifestations (Willison et al., 1993). The same antibody can be associated with different clinical symptoms, for example, antiGD1b antibodies via the NeuNAc( $\alpha 2-8) \operatorname{NeuNAc}(\alpha 2-3)$ moiety is associated with sensory neuropathy. The GD1b antibodies which react with antiGM1 via their common Gal( $\beta 1-3)$ GalNAc( $\beta 1-4)$ terminal may also be associated with the Guillain-Barre syndrome (Walsh et al., 1991) and multifocal motor neuropathy (Pestronk et al., 1988). The immunological cross reactivity together with the fine specificity of the antibodies probably produce the overlapping and variation in the clinical and immunological findings. The lack of immunoglobulin deposits in the sural nerve biopsies (Willison et al., 1993) despite the natural occurrence of GD1b and GT1b 
in peripheral nerve myelin (Fong et al., 1976) suggests that the antigenic site may be at another location. Immunostaining of the neuronal cytoplasm of rat dorsal root ganglia by $\lg M \lambda$ with antidisialosyl activity from a patient with large fibre sensory neuropathy was reported by Oka et al (1996). The blood vessels in the dorsal root ganglia are occasionally fenestrated whereas the endothelial cells in the endoneurium of peripheral nerve trunks are linked by tight junctions (King, 1999a). The blood-nerve barrier is therefore deficient in the dorsal root ganglia, permitting access by the paraproteins. The sensory deficits in these cases may be due to a sensory neuronopathy rather than neuropathy (Willison et al., 1993). Moreover in IgM antiMAG neuropathy the paraprotein clearly manages to enter peripheral nerve trunks. Against this is the fact that spontaneous or treatment-related improvement may occur which might not be expected in a neuronopathy.

The 6 patients who had an IgM paraproteinaemia with high anti$S$ titres do not conform to a single phenotype. In our series, all patients had a sensorimotor neuropathy with prominent large fibre deficits and significant motor involvement. One had an axonal neuropathy and 3 patients had prominent pain or dysaesthesia. Our experience confirms the observations of Lopate et al (1977a) those patients with high titres of anti-S and monoclonal gammopathy are more likely to have significant motor deficits, not to experience pain or dysaesthesia, and to have a demyelinating neuropathy. In contrast to the finding of Pestronk et al (1991), in the present series prominent pain was not confined to cases with axonal neuropathy. As sulphatide is found in abundance in the central nervous system and peripheral nerve myelin, it is not surprising that high titres of antisulphatide antibodies are found in a variety of conditions and not restricted to any single neurological disease (Lopate et al., 1997a; Toda et al., 1990; Van Viet et al., 1987), a particular neurological syndrome or type of neuropathy (Fredman et al., 1991; llyas et al., 1991; Nemni et al., 1993; van den Berg et al., 
1993). The only consistent finding in patients with neurological diseases is the presence of sensory impairment. Low levels of antisulphatide antibodies are found in normal individuals (Pestronk et al., 1991) and appear to be common constituents of the normal human immune repertoire and may be secreted by a CD5 subset of the B cells similar to the other naturally occurring $\operatorname{lgM}$ anticarbohydrate antibodies (Lee et al., 1991). These B cells may be activated by sulphatide incorporated into bacterial or viral envelopes in the course of infection (Pathak et al., 1990), by cross reactive foreign antigens or antigen complexes or by transformation in the monoclonal gammopathy (Latov, 1990b). The former is supported by the link between Campylobacter jejuni infection and antiGM1 antibodies by the demonstration of identical carbohydrate configurations (Aspinall et al., 1994; Yuki et al., 1993). An emerging association that may involve molecular mimicry is the occurrence of anti-GM2 antibodies in patients with Guillain-Barré syndrome with antecedent cytomegalovirus infection (Irie et al., 1996; Jacobs et al., 1997; Visser et al., 1996). A relationship between other strains of Campylobacter jejuni expressing disialosyl moieties in their lipopolysaccharides and the Miller Fisher variant of Guillain-Barré syndrome which is associated with antiGQ1b antibodies has also been established (Salloway et al., 1996; Yuki et al., 1994). Experiments in rodents also suggest that induction of antibodies to GM1 gangliosides may not be a direct response to Campylobacter jejuni but that the Campylobacter jejuni lipopolysaccharide selectively activates memory B cells from a previous exposure to cross reacting antigen (Wirguin et al., 1997).

Initiation of an immune response to foreign proteins according to Bretscher and Cohn (1970) requires 2 signals: signal 1 and signal 2. Initially the antigen presenting cell (APC), which can be a macrophage, or a dendritic cell, ingests protein antigens, cleaves them into peptides and presents the peptides "cradled" within the histocompatibility molecules. Signal 1 appears when an antigen displayed by the APC 
ligates the immunoreceptor on the helper $\mathrm{T}$ cell. Other molecules that come together during the contact between the 2 cells evoke signal 2 which is called a costimulatory signal. Ligation of the helper $T$ cell immunoreceptor without costimulatory signals can put the $T$ cell into a dormant state or "anergy" in which the lymphocyte will be unable to respond to the antigen. The effects of these signals are to induce the production of interleukin 2, activating the helper $T$ lymphocyte which can in turn induce $B$ cells or prepare cytotoxic $T$ cells for cell killing. Apart from interleukin 2, CTLA-4, a potent regulatory molecule which prevents the generation of the costimulatory signal and inhibits the growth of activated helper T cells, also appears on the surface of the helper T cell (Scheipers, Reiser, 1998). Whatever the mechanism, activated helper $T$ cells stop proliferating and die in the presence of CTLA-4. Conversely, in mice that lack CLTA-4, a rapidly fatal lymphoproliferative disorder of activated helper $T$ cell develops (Tivol et al., 1995). A solution of CTLA-4 can render $T$ cells anergic in vitro (Schwartz, 1992) and injections of soluble CTLA-4 allow animals to accept foreign grafts without the need for immunosuppression (Lin et al., 1993).

Despite the varied clinical neuropathic features, different immunocytochemical staining patterns (Lopate et al., 1997b) and the lack of immunoglobulins binding either in the patients' sural nerve biopsies (Nemni et al., 1993) or in rat sciatic nerve following intraneural injection (Quattrini et al., 1992), results from subsequent studies are supportive of a pathogenic role for antisulphatide antibodies. Ferrari et al (1998) were able to demonstrate evidence of demyelination, widely spaced myelin, C1q, C3d and the terminal complement complex C5bC9 not associated with $S$ protein in sural nerve biopsies of 2 patients with high titres of $\operatorname{lgM}$ activities against sulphatides. The presence of demyelination which was only evident by quantification of abnormalities on teased fibres in case 2 who has normal neurophysiological findings implies that binding of antisulphatide antibodies to the myelin target is 
not sufficient to induce electrophysiological or detectable ultrastructural change. The detection of $\mathrm{C} 1 \mathrm{q}$ suggests complement activation via the classical pathway whilst the absence of the $S$ protein bound to the terminal complement complex suggests that the complex is active and may be responsible for the structural abnormality. The finding of correlation in both number and location of fibres showing widely spaced myelin and the deposition of terminal complement complex indicates that in some cases, the peripheral neuropathy is caused by a complement dependent mechanism. Successful systemic passive transfer of antisulphatide antibodies to newborn rabbits produced demyelinating lesions (Nardelli et al., 1995) and experimental polyneuropathy produced in guinea pigs by immunization was characterised by weakness, demyelination and a correlation of functional deficit with levels of antisulphatide antibodies (Qin, Guan, 1997). The scope and the significance of antisulphatide antibodies in neurological diseases remain to be explored and demonstrated.

Three patients had both IgM antibody activities against MAG and sulphatide. This might reflect cross reactivity (llyas et al., 1992). Unfortunately an absorption test was not performed. Clinically and neurophysiologically they are indistinguishable from the $\mathrm{lgM}$ with antiMAG activity group apart from the aforementioned, relatively preserved distal CMAPs. Two patients had positive mitochondrial antibodies and positive ANCA, the latter with bilateral symmetrically delayed pattern reversal visual evoked potentials. No other patients in the whole series had positive autoantibodies. The association may occur stochastically but the findings raise the possibility of the antibodies reacting to the shared epitopes in the central as well as peripheral nervous system and to target tissues other than nerve components. MAG is known to be present both in the central and peripheral nervous system (Waxman, 1993). Moreover, while gel electrophoresis suggests differences in carbohydrate components, immunocytochemical studies of peripheral myelin show cross reaction 
with central nervous system MAG, both having a periaxonal distribution (Figlewicz et al., 1981; Sternberger et al., 1979). Because of the closely related epitopes, it is not unexpected that in some individuals there may be cross reactivity between the peripheral and central nervous system. Léger et al (1992b) reported $12-42 \%$ central nervous system involvement in 12 patients with peripheral neuropathy associated with benign IgM monoclonal gammopathy. The incidence varies according to the method used: $17 \%$ for abnormal magnetic resonance imaging of the brain, $42 \%$ for the detection of antiglycolipid antibodies to the central nervous system (which are not to sulphatide and which remain to be characterised), and $25 \%$ for abnormal central motor conduction time using magnetic brain stimulation. Most of the patients had IgM activities against MAG and SGPG. The coexistence of chronic inflammatory demyelinating polyneuropathy and multifocal central nervous system demyelination is well recognised (Mendell et al., 1987; Rubin et al., 1987; Thomas et al., 1987) but the underlying mechanisms in the 2 conditions are probably different.

Case 38 with HMSN1a and an IgM paraproteinaemic neuropathy together with endoneurial inflammatory infiltrates poses interesting questions. The identification of a chromosome 17p11.2 duplication confirms the diagnosis of HMSN $1 a$ in the absence of a family history. Positive sensory symptoms are unusual in HMSN1 (Harding, Thomas, 1980) and strongly suggest an additional process. The finding of an IgM band in the serum and the typical appearance of widely spaced myelin are consistent with a benign IgM paraproteinaemic neuropathy. The early onset of the sensory symptom at the age of 39 and the presence of endoneurial inflammatory infiltrates are atypical, raising the possibility of a superimposed inflammatory demyelinating polyneuropathy. This might be the result of chronic antigenic stimulation inducing proliferation of memory $B$ cells but why this should be monoclonal is problematic. Alternatively the demyelinating process might "unmask" epitopes which subsequently induce the development 
of a monoclonal gammopathy. In either way, the hypotheses would account for the late appearance of the monoclonal gammopathy. Dyck et al (1982) suggested that patients with HMSN are more likely to develop CIDP as a secondary immune reaction. Delayed appearance of an IgM paraprotein has been reported (Julien et al., 1984; Valldeoriola et al., 1993). This was observed in 2 other cases in the present series but the levels of the paraproteins were low and may have not been detected initially, as immunofixation was not performed. The hypothesis of chronic antigenic stimulation also extends to benign IgM paraproteinaemic neuropathy with antiMAG activity. This theory was supported by Gabriel et al (1998), who, by the use of multichannel confocal microscopy, reported an overlapping distribution of MAG and IgM deposits in the sural nerve biopsy of a patient with benign antiMAG positive demyelinating peripheral neuropathy of 6 mo duration. Serum antiMAG IgM antibodies were positive (using Western blot and ELISA techniques) whilst immunofixation at 6 and 12 mo showed no gammopathy. Serum IgM $\lambda$ remained undetected until 2 y at follow-up

Gibberd and Gavrilescu (1966) described a mother and a son with demyelinating neuropathy and a benign monoclonal gammopathy. The paraproteins migrated between beta and gamma globulin region and therefore could be either IgM or IgA (Turner, 1998). Another family of a mother and a son with peripheral neuropathy and benign IgM monoclonal gammopathy was reported by Busis et al (1985). Whilst the neurophysiological and pathological changes in the mother suggested a mixed demyelinating and axonal neuropathy, the findings in the son were typically demyelinating. MCV (nerve not specified) was less than $20 \mathrm{~m} / \mathrm{s}$. Sural nerve biopsy showed severe loss of myelinated fibres, widely spaced myelin and IgM deposits. The IgM was shown to have antiMAG activity.

In the light of the above, an underlying genetic defect therefore has to be entertained, at least in a few cases. As a series of remarkable genetic translocations naturally occurs within the pre $B$ cell 
series (Paul, 1988), it seems reasonable to hypothesise that the defect is related to the genes for the Ig variable region. Lastly a chance association cannot be excluded with certainty.

Patients with unidentified IgM antibody activity in the present series presented with a slowly progressive distal sensorimotor or predominantly large fibre sensory neuropathy with $50 \%$ incidence of tremor and/or pseudoathetosis and no facial or bulbar involvement. Although the symptoms are not dissimilar to those of the patients with antiMAG IgM paraproteinaemia, the findings on clinical examination were less severe. More than half of them had preserved tendon reflexes and only 1 case had thickened nerves. Seventeen percent had an axonal neuropathy and those with a demyelinating neuropathy had no evidence of distal accentuation but a high incidence of conduction block (67\%). In addition, $50 \%$ of cases retained their SAPs in the upper limbs at their last follow-up. Electrophysiologically this group of patients is more akin to patients with CIDP. The role of the IgM monocional gammopathy in these cases is uncertain. The antibody might react with some as yet unidentified nerve component or it might occur as an epiphenomenon.

Patients with IgG/A paraproteinaemic neuropathy were more likely to have facial symptoms or signs, more pronounced weakness in the upper limbs and often a remitting/relapsing course. They also had less severe overall neurological deficits on long term follow-up. In some cases improvement was observed clinically and neurophysiologically with and without treatment. In addition 25\% (3 out of 12 patients) had the pattern of abnormal median and normal sural sensory response. This group of patients differs from those with IgM paraproteinaemia and shares several common features with patients with CIDP (Notermans et al., 2000). Bleasel et al (1993) and Thomas and Willison (1994) concluded that these patients are identical in their clinical and neurophysiological features to chronic inflammatory demyelinating neuropathy. Simmons et al (1995) in their follow-up (mean 5-6 y) of 
patients with CIDP with and without monoclonal gammopathy found that patients who have CIDP and monoclonal gammopathy were more likely to have a chronic progressive course and were significantly poorer in strength and functional scores in comparison with those without a monoclonal gammopathy. They did not find any difference between the CIDP patients with IgM or IgG/A paraproteins. No IgM subgroup analysis was performed. Our patients with unidentified IgM antibody activities would fit with the patients of Simmons et al with CIDP and IgM paraproteinaemia. It is of interest to note that 2 out of 69 patients without monoclonal gammopathy subsequently developed monoclonal gammopathy and had to be reclassified.

None of our patient with IgG/A paraproteinaemic neuropathy had a pure axonal neuropathy. The neuropathy is usually mixed with both axonal and prominent demyelinating components including conduction block. The absence of instances of axonal neuropathy which are not uncommon in this disorder (Gorson, Ropper, 1997; Notermans et al., 1994) may be related to the small number of cases. To date the number of cases of IgA paraproteinaemic neuropathy is too small to make a separate comment. The ones that were reported appear to be heterogenous (Gherardi et al., 1993; Hemachudha et al., 1989; Simmons et al., 1993).

No patient in the present study was found to have antiGM1 activity or clinical manifestations of multifocal motor neuropathy or a lower motor neuron disease. The anti GM1 activity in such patients is rarely associated with a gammopathy (Kinsella et al., 1994; Miescher, Steck, 1996). A lively debate has arisen regarding the relationship between CIDP, MMN and the Lewis-Sumner syndrome. CIDP which is clinically described as a fairly symmetrical sensorimotor neuropathy, usually has predominantly motor involvement. Striking asymmetry can occur particularly at the onset. MMN, the termed coined by Pestronk et al (1990), is predominantly motor while Lewis-Sumner syndrome is best described as a multiple mononeuropathy with motor components 
predominating. Sensory involvement is ubiquitous in the Lewis-Sumner syndrome, and mild but consistent on quantitative sensory testing or in terms of abnormal SAPs (Bouche et al., 1995; Corse et al., 1996; Krarup et al., 1990; Nobile-Orazio et al., 1993) and nerve biopsy in MMN (Gibbels et al., 1993; Lewis et al., 1982). Widespread tendon areflexia is a common finding in all 3 conditions suggesting a more diffuse involvement of the afferent large sensory fibres $(A \alpha)$ from the muscle spindles. Although demyelination is more apparent in CIDP, all 3 conditions show multifocal demyelination with conduction block. Prolonged distal motor latencies, disproportionately prolonged $F$ waves, more widespread slowing of MCV in addition to focal motor conduction block can be seen in MMN and the Lewis-Sumner syndrome. Basic pathological changes are consistent with macrophage-mediated demyelination which only differs from each syndrome only by virtue of the distribution and the severity of sensory nerve involvement. Antiglycolipid antibodies, in particular antiGM1 antibodies are found in all even though more frequently and in higher in titres in MMN. Lastly the patients in all 3 groups respond to intravenous immunoglobulin therapy. Therefore these disorders share common features on clinical, electrophysiological, morphological and immunological grounds. The difference in the response to corticosteiods treatment reported by Saperstein et al (1999) is based on 6 patients with Lewis-Sumner syndrome only and no comparable treatment was given to the patients in the MMN group. Response to oral and intravenous corticosteroid therapy has been reported in patients with MMN (Bradley et al., 1988; Komiyama et al., 1998; Olney, Pestronk, 1992; van den Bergh et al., 1989). At present the evidence is insufficient for the distinction of the 3 disorders as discrete entities.

Recently Parry (1999) raised the question of reviewing the nosology of chronic demyelinating neuropathy and suggested dividing immune mediated neuropathy into axonal or demyelinating, either predominantly sensory or motor and diffuse, widespread but multifocal 
or restricted in distribution. As immunology has advanced along with molecular biology, should one go one step further by using the identifiable antibody activities for classification? This should give more insight into the disease process and serve as a reminder that the final clinical manifestations depend on the complex and dynamic processes involving the network of the immune system, cross reactivity, fine specificity of antibody activity, and the location and accessibility of the antigenic sites which could be triggered by environmental factors and/or genetic defects. With the encouraging results from the pioneer study of Guinan et al (1999) on bone marrow transplantation employing partially histocompatible donors and applying the principle of anergy using CTLA-4 inhibitory effect on T cells in the absence of costimulatory signals (Schwartz, 1999), the future for the 'new immunotherapy' replacing immunosuppressive treatment might not be so far away. 
CHAPTER 5

CONCLUSIONS 


\section{SUMMARY}

The primary aim of the study was to determine the long term clinical outcome, including the incidence of haematological malignancy, in a group of patients initially diagnosed as having neuropathy associated with a benign monoclonal gammopathy. The evolution of the neurophysiological findings over time was also examined. An attempt was made to delineate individual nosological subgroups based on the clinical course and the immunological and neurophysiological findings.

Fifty patients with peripheral neuropathy associated with benign monoclonal gammopathy were identified from hospital records. Fifteen had died. The remainder were followed up clinically and electrophysiologically along with 5 patients who satisfied full diagnostic criteria for CIDP. The latter had no gammopathy on repeated blood testing. The duration of the follow-up period was 5 to $41 \mathrm{y}$. Sera were also taken for paraprotein quantification and for antiMAG and antiganglioside antibodies, and if indicated, cold agglutinin antibodies. Scoring of the clinical symptoms and signs was performed as described by Dyck et al (1980) and Notermans et al (1994).

Most of the patients $(74.5 \%)$ with benign monoclonal gammopathy had IgM paraproteins, predominantly with a $\kappa$ light chain (64\%). They had a high M:F ratio (18:7). Only a single case with an IgA paraprotein was identified. The onset of symptoms was usually in the latter part of the fifth decade and was significantly earlier in the CIDP and in the IgM subgroup with antidisialosyl activities. Amongst the subgroups with IgM paraproteins (limited to the patients who were alive), $29 \%$ were antiMAG positive, $12.5 \%$ had both antiMAG and anti-S activity, $8.3 \%$ had antidisialosyl antibodies, and $25 \%$ had anti-S or unidentified antibody activities.

Only $6 \%$ of patients developed haematological malignancies in comparison with the reported $17-25 \%$ in patients ascertained by the 
presence of a paraprotein in the absence of neuropathy (Kyle, 1995). The haematological malignancies were predominantly $\mathrm{B}$ cell related. None developed multiple myeloma. The interval from the detection of the paraprotein to diagnosis of haematological malignancy ranged from 11 to $25 \mathrm{y}$ and in the only case with detailed information, this was preceded by a sudden rise in the paraprotein level associated with malaise. All these patients had been on immunosuppressive therapy for at least 2 y prior to the diagnosis of malignancy. The highest incidence of malignancy of all types ( $44 \%$ in the present series) was of the gastrointestinal tract.

No risk factors for haematological malignancy could be identified using a Kaplan-Meier log rank test at a $90 \%$ confidence level. The possibility exists the malignant change was precipitated by immunosuppressive therapy but the numbers were too small for statistical evaluation.

Cases of paraproteinaemic neuropathy with $\operatorname{lgM}$ antiMAG and antidisialosyl antibodies have consistent clinical and serological associations. The patients with IgM antiMAG paraproteinaemic neuropathy were predominantly male and usually presented with a late onset distal sensorimotor neuropathy. Initially large sensory fibres were involved affecting in particular, vibration sense. Postural tremor and pseudoathetosis were common early features, mainly affecting the upper limbs, and postural tremor could occur in the face of normal joint position sense. Dysaesthesia or prominent pain was present in $45 \%$ and peripheral nerve thickening in $28 \%$. There was no facial/bulbar or autonomic involvement. Progression of the neuropathy was always slow and steady in the present series with significant motor and sensory deficits in comparison with the other groups of patients. In some cases the tremor was severely disabling. Prominent pain or dysaesthesia resolved in all cases after 3 to $15 \mathrm{y}$.

Nerve conduction studies in patients with IgM antiMAG paraproteinaemic neuropathy revealed a demyelinating neuropathy in 
all cases. Motor conduction velocity was slow, particularly in the very distal segments in the initial stages. More pronounced peripheral slowing was evident by relatively prolonged distal latencies in comparison with motor conduction velocities in the more proximal segments where slowing appeared to be more uniform. Conduction block was rare. Despite stable maximal motor conduction velocity after $5 \mathrm{y}$, serial electromyographic examination and distal CMAP amplitude recordings showed a progressive change indicating axonal degeneration. The relative prolongation of the distal motor latencies was no longer evident after this lengthy follow-up period suggesting centripetal progression of demyelination or secondary axonal degeneration leading to normalisation of the terminal latency index from loss of severely affected fibres. The latter explanation is supported by the significantly reduced mean distal CMAP amplitude in this group of patients.

A small number of patients (12.5\%) had an IgM paraproteinaemic neuropathy with both antiMAG and anti-S activity. Clinically and electrophysiologically they were indistinguishable from those with antiMAG activity alone apart from the relatively preserved distal CMAP amplitudes at the last follow-up. Of interest was a high incidence $(67 \%)$ of positive titres for miscellaneous autoantibodies. In 1 of these patients there were also bilateral symmetrically delayed pattern visual evoked potentials.

Two $(8 \%)$ of the patients with benign IgM paraproteinaemic neuropathy had antibody activity against disialosyl groups. Both displayed the clinical and serological phenotype of CANOMAD. These patients presented in early midlife with fluctuating oculofacial and/or bulbar symptoms on a background of a slowly progressive ataxic large fibre sensorimotor neuropathy. In 1 patient, subacute deterioration resembling the Miller-Fisher syndrome following an operation for hip replacement, responded dramatically to intravenous immunoglobulin therapy. The other patient also had transient but significant 
improvement with the same treatment. Neurophysiological studies were consistent with asymmetric demyelination with conduction block.

A quarter of patients with IgM paraproteins had high titres of anti$S$ antibody. All patients had a steadily progressive sensorimotor neuropathy with predominantly large fibre involvement and significant motor deficits. Facial symptoms occurred in $33 \%$ and prominent pain or dysaesthesia was present in $50 \%$. Neurophysiological studies were consistent with axonal degeneration in $17 \%$ whilst the rest had a demyelinating neuropathy. Conduction block was evident in $17 \%$. Prominent pain or dysaesthesia was associated with neurophysiological changes either of axonal or demyelinating neuropathy.

About the same number of patients with IgM paraproteinaemic neuropathy as in the anti-S subgroup had unidentified antibody activity. Although the symptoms and clinical progression of the patients in this group were not dissimilar to those with the antiMAG IgM paraproteinaemia, the findings on clinical examination were less severe. More than half had preserved tendon reflexes and only 1 case had thickened nerves. Those with a demyelinating neuropathy had no evidence of distally accentuated slowing of nerve conduction and there was a $67 \%$ incidence of conduction block. In addition, $50 \%$ of cases had retained SAPs in their upper limbs at their last follow-up. The majority of patients in this group with a demyelinating neuropathy showed neurophysiological changes akin to patients with CIDP. Only a small number (17\%) had an axonal neuropathy.

Patients with IgG/A paraproteinaemic neuropathy were more likely to have facial symptoms or signs and more pronounced weakness in the upper limbs. A relapsing and remitting but generally progressive course was evident in more than half $(58 \%)$. The relapses or remissions occurred spontaneously in $17 \%$ and followed a viral infection in $17 \%$, whilst in the rest relapses occurred either on withdrawal or reduction of steroid therapy. This was the only group of patients in which improvement in upper limb MCV was seen. None of the patients 
in this group in the present series had a pure axonal neuropathy. The neuropathy was usually mixed axonal and demyelinating with a prominent demyelinating component, including conduction block in $40 \%$. In general, this group behaved like patients with CIDP.

In conclusion the patients with IgM antiMAG and antidisialosyl antibodies each formed separate clinical and immunological entities. Patients with an IgM paraprotein and high anti-S titres had a less consistent clinical pattern whereas patients with IgM paraproteins with unidentified antibody activity and those with IgG/A paraproteins were clinically and electrophysiologically heterogeneous. 


\section{REFERENCES}

ABRAMS GM, LATOV N, HAYS AP, SHERMAN W, ZIMMERMAN EA (1982) Immunocytochemical studies of human peripheral nerve with serum from patients with polyneuropathy and paraproteinemia. Neurology, 32, 821-826.

AD HOC SUBCOMMITTEE OF THE AMERICAN ACADEMY OF NEUROLOGY AIDS TASK FORCE (1991) Research criteria for diagnosis of chronic inflammatory demyelinating neuropathy (CIDP). Neurology, 41, 617-618.

AMEIS A, KO HS, PRUZANSKI W (1976) M components-a review of 1242 cases. Can Med Assoc J, 114, 889-892,895.

ANTOINE JC, STECK A, MICHEL D (1993) Neuropathie peripherique mortelle a predominance mortice associeé a une IgM monoclonale anti-MAG. Rev Neurol(Panis), 149, 496-499.

AQUINO DA, MARGOLIS RU, MARGOLIS RK (1984a) Immunocytochemical localization of a chondroitin sulfate proteoglycan in nervous tissue. I. Adult brain, retina and peripheral nerve. J Cell Biol, 99, 1117-1129.

AQUINO DA, MARGOLIS RU, MARGOLIS RK (1984b) Immunocytochemical localization of a chondroitin sulfate proteoglycan in nervous tissue. II. Studies in developing brain. J Cell Biol, 99, 1139

ARAI M, YOSHINO $H$, KUSANO $Y$, YAZAKI $Y$, OHNISHI $Y$, MIYATAKE T (1992) Ataxic polyneuropathy and anti-Pr2 IgM kappa M proteinemia. J Neurol, 239, 147-151.

ARIGA T, KOHRIYAMA T, FREDDO L, LATOV $N$, SAITO $M, K O N ~ K$ et al (1987) Characterization of sulfated glucuronic acid containing glycolipids reacting with IgM M-proteins in patients with neuropathy. $J$ Biol Chem, 262, 848-853. 
ASPINALL GO, MCDONALD AG, PANG H, KURJANCZYK LA, PENNER JL (1994) Lipopolysaccharides of Campylobacter jejuni serotype 0:19: structures of core oligosaccharide regions from the serostrain and two bacterial isolates from patients with the Guillain-Barré syndrome. Biochemistry, 33, 241-249.

AUER RN, BELL RB, LEE MA (1989) Neuropathy with onion bulb formations and pure motor manifestation. Can J Neurol Sci, 16, 194-197.

AXELSSON U, BACHMANN R, HALLEN J (1966) Frequency of pathological proteins ( $M$ components) in 6995 sera from adult population. Acta Med Scand, 179, 235-247.

AZAR HA, HILL WT, OSSERMAN EF (1957) Malignant lymphoma and lymphatic leukemia associated with myeloma-like serum proteins. Am J Med, 23, 239-249.

BABA $H$, DUANE GC, ILYAS AA, PESTRONK A, CORNBLATH D, CHAUDHRY $V$, et al (1989) Anti-GM1 ganglioside antibodies with differing fine specificities in patients with multifocal motor neuropathy. J Neuroimmunol, 25, 143-150.

BAILEY RO, RITACCIO AL, BISHOP MB, WU AY (1986) Benign monoclonal IgAK gammopathy associated with polyneuropathy and dysautonomia. Acta Neurol Scand, 73, 574-580.

BAIN PG, BRITTON TC, JENKINS IH, THOMPSON PD, ROTHWELL JC, THOMAS PK et al (1996) Tremor associated with benign IgM paraproteinaemic neuropathy. Brain, 119, 789-799.

BAUR PS, STACEY TR (1977) The use of PIPES buffer in the fixation of mammalian and marine tissues for electron microscopy. J Microsc, 109, 315-327.

BLADE J, LOPEZ-GUILLERMO A, ROZMAN C, CERVANTES F, SALGADO C, AQUILAR JL et al (1992) Malignant transformation and life 
expectancy in monoclonal gammopathy of undetermined significance. Br J Haematol, 81, 391-394.

BLEASEL AF, HAWKE SH, POLLARD JD, MCLEOD JG (1993) IgG monoclonal paraproteinaemia and peripheral neuropathy. J Neurol Neurosurg Psychiatry, 56, 52-57.

BOLLENSEN E, STECK AJ, SCHACHNER M (1988) Reactivity with the peripheral myelin glycoprotein Po in serum from patients with monoclonal IgM gammopathy and polyneuropathy. Neurology, 38, 1266-1270.

BOSCH EP, ANSBACHER LE, GOEKEN JA, CANCILLA PA (1982) Peripheral neuropathy associated with monoclonal gammopathy. Studies of intraneural injections of monoclonal immunoglobulin sera. $J$ Neuropathol Exp Neurol, 41, 446-459.

BOUCHE P, MOULONGUET A, YOUNES-CHENNOUFI AB, ADAMS D, BAUMANN N, MEININGER V, et al (1995) Multifocal motor neuropathy with conduction block: a study of 24 patients. J Neurol Neurosurg Psychiatry, 59, 38-44.

BRADLEY WG, BENNETT RK, GOOD P, LITTLE B (1988) Proximal chronic inflammatory polyneuropathy with multifocal conduction block. Arch Neurol, 45, 451-455.

BRAUN PE, FRAIL DE, LATOV N (1982) Myelin-associated glycoprotein is the antigen for a monoclonal $\lg M$ in polyneuropathy. J Neurochem, 39, 1261-1265.

BRETSCHER P, COHN M (1970) A theory of self-nonself discrimination. Science, 169, 1042-1049.

BRIANI C, BERGER JS, LATOV N (1998) Antibodies to chondroitin sulfate C: a new detection assay and correlations with neurological diseases. $J$ Neuroimmunol, 84, 117-121. 
BROMBERG MB, ALBERS JW (1993) Patterns of sensory nerve conduction abnormalities in demyelinating and axonal peripheral nerve disorders. Muscle Nerve, 16, 262-266.

BROOKS DJ, JENKINS IH, BAIN PG, COLEBATCH JG, THOMPSON PD, FINDLEY LJ, et al (1992) A comparison of the abnormal patterns of cerebral activation associated with neuropathic and essential tremor. Neurology, 42 supplement 3, 423

BUSIS NA, HALPERIN JJ, STEFANSSON K, KWIATKOWSKI DJ, SAGAR SM, SCHIFF SR et al (1985) Peripheral neuropathy, high serum IgM, and paraproteinemia in mother and son. Neurology, 35, 679-683.

CHAD DA, HAMMER K, SARGENT J (1986) Slow resolution of multifocal weakness and fasciculation: a reversible motor neuron syndrome. Neurology, 36, 1260-1263.

CHASSANDE B, LEGER JM, YOUNES-CHENNOUFI AB, BENGOUFA D, MAISONOBE T, BOUCHE $P$ et al (1998) Peripheral neuropathy associated with IgM monoclonal gammopathy: correlations between $\mathrm{M}$ protein antibody activity and clinical/electrophysiological features in $\mathbf{4 0}$ cases. Muscle Nerve, 21, 198-205.

CHAUDHRY V, CORSE AM, CORNBLATH DR, KUNCL RW, FREIMER ML, GRIFFIN JW (1994) Multifocal motor neuropathy: electrodiagnostic features. Muscle Nerve, 17, 198-205.

CHOU DK, ILYAS AA, EVANS JE, COSTELLO C, QUARLES RH, JUNGALWALA FB (1986) Structure of sulfated glucuronyl glycolipids in the nervous system reacting with HNK-1 antibody and some IgM paraproteins in neuropathy. J Biol Chem, 261, 11717-11725.

COLEBATCH JG, FINDLEY LJ, FRACKOWIAK RSJ, MARSDEN CD, BROOKS DJ (1990) Preliminary report: activation of the cerebellum in essential tremor. Lancet, 336, 1028-1030. 
CONNOLLY AM, PESTRONK A, TROTTER JL, FELDMAN EL, CORNBLATH DR, OLNEY RK (1993) High-titer selective serum anti-beta-tubulin antibodies in chronic inflammatory demyelinating polyneuropathy. Neurology, 43, 557-562.

CONNOLLY AM, PESTRONK A (1997) Anti-tubulin autoantibodies in acquired demyelinating polyneuropathies. J Infect Dis, 176(suppl 2), S157S159

CORNBLATH DR, SUMNER AJ, DAUBE J, GILLIATT RW, BROWN WF, PARRY GJ, et al (1990) Conduction block in clinical practice. Muscle Nerve, 14, 869-871.

CORSE AM, CHAUDHRY V, CRAWFORD TO, CORNBLATH DR, KUNCL RW, GRIFFIN JW (1996) Sensory nerve pathology in multifocal motor neuropathy. Ann Neurol, 39, 319-325.

DALAKAS MC, ENGEL WK (1981) Polyneuropathy with monoclonal gammopathy: studies of 11 patients. Ann Neurol, 10, 45-52.

DALAKAS MC, TERAVAINEN H, ENGEL WK (1984) Tremor as a feature of chronic relapsing and dysgammoglobulinemic polyneuropathies. Incidence and management. Arch Neurol, 41, 711-714.

DELLAGI K, BROUET JC, PERREAU J, PAULIN D (1982) Human monoclonal $\operatorname{lgM}$ with autoantibody activity against intermediate filaments. Proc Natl Acad Sci USA, 79, 446-450.

DUANE GC, FARRER RG, DALAKAS MC, QUARLES RH (1992) Sensory neuropathy associated with monoclonal immunoglobulin $M$ to GD1b ganglioside. Ann Neurol, 31, 683-685.

DYCK PJ, SHERMAN WR, HALLCHER LM, SERVICE JF, O'BRIEN PC, GRINA LA et al (1980) Human diabetic endoneurial sorbitol, fructose, and myo-inositol related to sural nerve morphometry. Ann Neurol, 8, 590-596. 
DYCK PJ, SWANSON CJ, LOW PA, BARTLESON JD, LAMBERT EH (1982)

Prednisone-responsive hereditary motor and sensory neuropathy. Mayo Clin Proc, 57, 239-246.

DYCK PJ, LOW PA, WINDEBANK AJ, JARADEH SS, GOSSELIN S, BOURQUE $P$, et al (1991) Plasma exchange in polyneuropathy associated with monoclonal gammopathy of undetermined significance. N Eng J Med, 325, 1482-1486.

ELLIE E, VITAL A, STECK AJ, JULIEN J, HENRY P, VITAL C (1995) Highgrade B-cell cerebral lymphoma in a patient with anti-myelinassociated glycoprotein IgM paraproteinemic neuropathy. Neurology, 45, 378-381.

ELLIE E, VITAL A, STECK A, BOIRON JM, VITAL C, JULIEN J (1996) Neuropathy associated with "benign" anti-myelin-associated glycoprotein IgM gammopathy: clinical, immunological, neurophysiological, pathological findings and response to treatment in 33 cases. J Neurol, 243, 34-43.

FAZIO R, NEMNI R, QUATTRINI A, LORENZETTI I, CANAL N (1992) IgG monoclonal proteins from patients with axonal peripheral neuropathies bind to different epitopes of the $68 \mathrm{kDa}$ neurofilament protein. $J$ Neuroimmunol, 36, 97-104.

FELDMAN EL, BROMBERG MB, ALBERS JW, PESTRONK A (1991) Immunosuppressive treatment in multifocal motor neuropathy. Ann Neurol, 30, 397-401.

FERRARI S, MORBIN M, NOBILE-ORAZIO E, MUSSO A, TOMELLERI G, BERTOLASI L et al (1998) Antisulfatide polyneuropathy: antibodymediated complement attack on peripheral myelin. Acta Neuropathol(Ber), 96, 569-574.

FIGLEWICZ DA, QUARLES RH, JOHNSON D, BARBARASH GR, STERNBERGER NH (1981) Biochemical demonstration of the myelin- 
assoicated glycoprotein in the peripheral nervous system. $J$ Neurochem, 37, 749-758.

FINE JM, LAMBIN P, LEROUX P (1978) Frequency of monoclonal gammopathy (M-components) in 13,400 sera from blood donors. Vox Sang, 23, 343

FINEMAN SM, MCKENDELL RR (1990) Plasma exchange: a treatment for neuropathy associated with IgG-kappa gammopathy. J Neurol, 237, 85-87.

FITTING JW, BISCHOFF A, REGLI F, DE CROUSAZ G (1979) Neuropathy, amyloidosis, and monoclonal gammopathy. $J$ Neurol Neurosurg Psychiatry, 42, 193-202.

FONG JW, LEDEEN RW, KUNDU SW, BROSTOFF SW (1976) Gangliosides of peripheral nerve myelin. J Neurochem, 26, 157-162.

FORSSMAN O, BJÖRKMAN G, HOLLENDER A, ENGLUND NE (1973) IgMproducing lymphocytes in peripheral nerve in a patient with benign monoclonal gammopathy. Scand J Haematol, 11, 332-335.

FREDDO L, YU RK, LATOV N, DONOFRIO PD, HAYS AP, GREENBERG HS et al (1986) Gangliosides GM1 and GD1b are antigens for IgM Mprotein in a patient with motor neuron disease. Neurology, 36, 454458.

FREDMAN P, VEDELER CA, NYLAND $H$, AARLI JA, SVENNERHOLM L (1991) Antibodies in sera from patients with inflammatory demyelinating polyradiculoneuropathy react with ganglioside LM1 and sulphatide of peripheral nerve myelin. J Neurol, 238, 75-79.

GABRIEL JM, ERNE B, MIESCHER GC, MILLER SL, VITAL A, VITAL C, et al (1996) Selective loss of myelin-associated glycoprotein from myelin correlates with anti-MAG antibody titre in demyelinating paraproteinaemic polyneuropathy. Brain, 119, 775-787. 
GABRIEL JM, ERNE B, BERNASCONI L, TOSI C, PROBST A, LANDMANN L et al (1998) Confocal microscopic localization of anti-myelin-associated glycoprotein autoantibodies in a patient with peripheral neuropathy initially lacking a detectable IgM gammopathy. Acta Neuropathol(Ber), 95, 540-546.

GHERARDI RK, MALAPERT D, DEGOS J-D (1993) Polyneuropathy associated with $\operatorname{lgA}$ monoclonal gammopathy of undetermined significance. Muscle Nerve, 16, 1129-1130.

GIBBELS E, BEHSE F, KENTENICH M, HAUPT WF (1993) Chronic multifocal neuropathy with persistent conduction block (Lewis-Sumner syndrome). A clinico-morphologic study of two further cases with review of the literature. Clin Neuropathol, 12, 343-352.

GIBBERD FB, GAVRILESCU K (1966) A familial neuropathy associated with a paraprotein in the serum, cerebrospinal fluid, and urine. Neurology, 16, $130-134$.

GIRALDO MP, RUBIO-FÉLIX D, PERELLA M, GRACIA JA, BERGUA JM, GIRALT M (1991) Gammapatias monoclonales de significado indeterminado. Aspectos clínicos, biológicos y evolutivos de 397 casos. Sangre(Barc), 36, 377-382.

GORSON KC, ROPPER AH (1997) Axonal neuropathy associated wtih monoclonal gammopathy of undetermined significance. I Neurol Neurosurg Psychiatry, 63, 163-168.

GOSSELIN S, KYLE R, DYCK PJ (1991) Neuropathy associated with monoclonal gammopathies of undetermined significance. Ann Neurol, 30, 54-61.

GREGORY R, THOMAS PK, KING RH, HALLAM PL, MALCOLM S, HUGHES $R A$, et al (1993) Coexistence of hereditary motor and sensory neuropathy type la and IgM paraproteinemic neuropathy. Ann Neurol, 33, 649-652. 
GUINAN EC, BOUSSIOTIS VA, NEUBERG D, BRENNAN LLV, HIRANO N, NADLER LM et al (1999) Transplantation of anergic histoincompatibility bone marrow allografts. N Eng J Med, 340, 17041714.

HAFLER DA, JOHNSON D, KELLY JJ, PANITCH H, KYLE R, WEINER HL (1986) Monoclonal gammopathy and neuropathy: myelin-associated glycoprotein reactivity and clinical characteristics. Neurology, 36, 7578.

HANSEN PR, JØNSSONN V, SCHRØDER HD, JENSEN TS, WIIK A (1989) $\operatorname{lgD}-\lambda$ monoclonal gammopathy and axonal neuropathy. $J$ Int Med, 225, 289-290.

HARDING AE, THOMAS PK (1980) The clinical features of hereditary motor and sensory neuropathy type I and II. Brain, 103, 259-280.

HAYS AP, LATOV N, TAKATSU M, SHERMAN WH (1987) Experimental demyelination of nerve induced by serum of patients with neuropathy and an anti-MAG IgM M-protein. Neurology, 37, 242-256.

HAYS AP, ROXAS A, SADIQ SA, VALLEJOS H, D'AGATI V, THOMAS FP, et al (1990) A monoclonal IgA in a patient with amyotrophic lateral sclerosis reacts with neurofilaments and surface antigen on neuroblastoma cells. J Neuropathol Exp Neurol, 49, 383-398.

HEMACHUDHA T, PHANUPHAK $\mathrm{P}$, PHANTHUMCHINDA $\mathrm{K}$, KASEMPIMOLPORN S (1989) Proximal motor neuropathy, IgA paraproteinaemia and anti-myelin-associated glycoprotein reactivity. Postgrad Med J, 65, 662-664.

HERRON B, WILLISON HJ, VEITCH J, ROELCKE D, ILLIS LS, BOULTON FE (1994) Monoclonal IgM cold agglutinins with anti-Pr1d specificity in a patient with peripheral neuropathy. Vox Sang, 67, 58-63. 
HUGHES RA, NEWSOM-DAVIS JM, PERKINS GD, PIERCE JM (1978) Controlled trial of prednisolone in acute polyneuropathy. Lancet, 2, 750-753.

ILYAS AA, QUARLES RH, MACINTOSH TD, DOBERSEN MJ, TRAPP BD, DALAKAS MC et al (1984) IgM in a human neuropathy related to paraproteinemia binds to a carbohydrate determinant in the myelinassociated glycoprotein and to a ganglioside. Proc Natl Acad Sci USA, 81, 1225-1229.

ILYAS AA, QUARLES RH, DALAKAS MC, FISHMAN PH, BRADY RO (1985) Monoclonal IgM in a patient with paraproteinemic polyneuropathy binds to gangliosides containing disialosyl groups. Ann Neurol, 18, 655-659.

ILYAS AA, DALAKAS MC, BRADY RO, QUARLES RH (1986a) Sulfated glucuronyl glycolipids reacting with anti-myelin-associated glycoprotein monoclonal antibodies including $\lg \mathrm{M}$ paraproteins in neuropathy: species distribution and partial characterization of epitopes. Brain Res, $385,1-9$.

ILYAS AA, DOBERSEN MJ, WILLISON HJ, QUARLES RH (1986b) Mouse monoclonal and rabbit polyclonal antibodies prepared to human myelin-associated glycoprotein also react with glycosphingolipids of peripheral nerve. J Neuroimmunol, 12, 99-106.

ILYAS AA, WILLISON HJ, DALAKAS MC, WHITAKER JN, QUARLES RH (1988) Identification and characterization of gangliosides reacting with IgM paraproteins in three patients with neuropathy associated with biclonal gammopathy. J Neurochem, 51, 851-858.

ILYAS AA, CHOU DK, JUNGALWALA FB, COSTELLO C, QUARLES RH (1990) Variability in the structural requirements for binding of human monoclonal anti-myelin-associated glycoprotein immunoglobulin $M$ antibodies and HNK-1 to sphingoglycolipid antigens. J Neurochem, 55, 594-601. 
ILYAS AA, MITHEN FA, DALAKAS M, WARGO M, CHEN Z-W, BIELORY L, et al (1991) Antibodies to sulfated glycolipids in Guillain-Barré syndrome. J Neurol Sci, 105, 108-117.

ILYAS AA, COOK SD, DALAKAS MC, MITHEN FA (1992) Anti-MAG IgM paraproteins from some patients with polyneuropathy associated with IgM paraproteinemia also react with sulfatide. J Neuroimmunol, 37, 85-92.

IRIE S, SAITO T, NAKAMURA K, KANAZAWA N, OGINO M, NUKAZAWA T, et al (1996) Association of anti-GM2 antibodies in Guillain-Barré syndrome with acute cytomegalovirus infection. J Neuroimmunol, 68, 19-26.

ISAKSSON E, BJORKHOLM M, HOLM G, JOHANSSON B, NILSSON B, MELLSTEDT $\mathrm{H}$ et al (1996) Blood clonal B-cell excess in patients with monoclonal gammopathy of undetermined significance (MGUS): association with malignant transformation. Br J Haematol, 92, 71-76.

JACOBS BC, O'HANLON GM, BREEDLAND EG, VEITCH J, VAN DOORN PA, WILLISON HJ (1997) Human IgM paraproteins demonstrate shared reactivity between Campylobacter jejuni lipopolysaccharides and human peripheral nerve disialylated gangliosides. $J$ Neuroimmunol, 80, 23-30.

JACOBS JM, SCADDING JW (1990) Morphological changes in IgM paraproteinaemic neuropathy. Acta Neuropathol, 80, 77-84.

JACOBS JM, GREGORY RA (1991) Uncompacted lamellae as a feature of tomaculous neuropathy. Acta Neuropathol(Berl), 83, 87-91.

JACOBS JM (1996) Morphological changes at paranodes in IgM paraproteinaemic neuropathy. Microsc Res Tech, 34, 544-553.

JENKINS IH, BAIN PG, COLEBATCH JG, THOMPSON PD, FINDLEY LJ, FRACKOWIAK RS, et al (1993) A positron emission tomography study 
of essential tremor: evidence for overactivity of cerebellar connections. Ann Neurol, 34, 82-90.

JULIEN J, VITAL C, VALLAT JM, LAGUENY A, FERRER X, LEBOUTET MJ (1984) Chronic demyelinating neuropathy with IgM-producing lymphocytes in peripheral nerve and delayed appearance of "benign" monoclonal gammopathy. Neurology, 34, 1387-1389.

KAHN SN, RICHES PG, KOHN J (1980) Paraproteinaemia in neurological disease: incidence, associations, and classification of monoclonal immunoglobulins. J Clin Pathol, 33, 617-621.

KAHN SN, SMITH IA, EAMES RA, THOMAS PK, LACEY BW (1981) IgM paraproteinemia and autoimmune peripheral neuropathy. $N$ Engl $J$ Med, 304, 1430-1431.

KAJI R, SHIBASAKI H, KIMURA J (1992) Multifocal demyelinating motor neuropathy:cranial nerve involvement and immunoglobulin therapy. Neurology, 42, 506-509.

KAKU DA, SUMNER AJ (1992) Characteristic electrophysiological findings in anti-MAG polyneuropathy. Neurology, 42(supplement 3), 408.

KAKU DA, ENGLAND JD, SUMNER AJ (1994) Distal accentuation of conduction slowing in polyneuropathy associated with antibodies to myelin-associated glycoprotein and sulphated glucuronyl paragloboside. Brain, 117, 941-947.

KATZ JS, WOLFE GI, BRYAN WW, JACKSON CE, AMATO AA, BAROHN RJ (1997) Electrophysiologic findings in multifocal motor neuropathy. Neurology, 48, 700-707.

KEIR G, LUXTON RW, THOMPSON EJ (1990) Isoelectric focusing of cerebrospinal fluid IgG: an annotated update. Ann Clin Biochem, 27, 436-443. 
KELLY JJ, Jr., KYLE RA, O'BRIEN PC, DYCK PJ (1981) Prevalence of monoclonal gammopathy in peripheral neuropathy. Neurology, 31, 1480-1483.

KELLY JJ, Jr. (1985) Peripheral neuropathies associated with monoclonal proteins: a clinical review. Muscle Nerve, 8, 138-150.

KELLY JJ, Jr. (1990) The electrodiagnostic findings in polyneuropathies associated with IgM monoclonal gammopathies. Muscle Nerve, 13, 1113-1117.

KING RHM, THOMAS PK (1984) The occurrence and significance of myelin with unusually large periodicity. Acta Neuropathol(Berlin), 63, 319329.

KING RHM (1999a) Normal structure. In: King RHM. Atlas of penipheral nerve pathology. London: Arnold, p 6.

KING RHM (1999b) Pathological changes:myelin abnormalities. In: King RHM Atlas of peripheral nerve pathology. London: Arnold, $\mathrm{p} 113$.

KINSELLA LJ, LANGE DJ, TROJABORG W, SADIQ SA, YOUNGER DS, LATOV N (1994) Clinical and electrophysiologic correlates of elevated anti-GM1 antibody titers. Neurology, 44, 1278-1282.

KOMIYAMA A, TODA H, HASEGAWA O, KUROIWA $Y$ (1998) Multifocal motor neuropathy. Neurology, 50, 314-315.

KRARUP C, STEWART JD, SUMNER AJ, PESTRONK A, LIPTON SA (1990) A syndrome of asymmetric limb weakness with motor conduction block. Neurology, 40, 118-127.

KYLE RA, NOBREGA FT, KURLAND LT (1969) Multiple myeloma in Olmstead county, Minnesota, 1945-1964. Blood, 33, 739-745.

KYLE RA, FINKELSTEIN S, ELVEBACK LR, KURLAND LT (1972) Incidence of monoclonal proteins in a Minnesota community with a cluster of multiple myeloma. Blood, 40, 719-724 
KYLE RA (1978) Monoclonal gammopathy of undetermined significance. Natural history in 241 cases. Am J Med, 64, 814-826.

KYLE RA (1988) Plasma cell disorders. In: R Cecil editor. Intemal Medicine. Philadelphia: W.B.Saunders Company, pp. 967-978.

KYLE RA, DYCK PJ (1993) Neuropathy associated with monoclonal gammopathies. In: Peripheral Neuropathy. Edited by PJ Dyck, PK Thomas, JW Griffin, PA Low, JF Poduslo. Philadelphia: WB Saunders, pp. 1275-1287.

KYLE RA (1995) Monoclonal gammopathy of undetermined significance (MGUS). Baillière's Clin Neurol, 8, 761-781.

LANGE DJ, TROJABORG W, LATOV N, HAYS AP, YOUNGER DS, UNCINI A et al (1992) Multifocal motor neuropathy with conduction block: is it a distinct clinical entity? Neurology, 42, 497-505.

LATOV N, SHERMAN WH, NEMNI R, GALASSI G, SHYONG JS, PENN AS, et al (1980) Plasma cell dyscrasia and peripheral neuropathy with a monoclonal antibody to peripheral nerve myelin. N Engl J Med, 303 , 618-621.

LATOV N, HAYS AP, SHERMAN WH (1988) Peripheral neuropathy and antiMAG antibodies. CRC Crit Rev Neurobiology, 3, 301-332.

LATOV N (1990a) Antibodies to glycoconjugates in neurological disease. Clin Aspects Autoimmun, 4, 18-29.

LATOV N (1990b) Neuropathic syndromes associated with monoclonal gammopathies. Res Publ Assoc Res Nerv Ment Dis, 68, 221-232.

LATOV N (1994) Antibodies to glycoconjugates in neuropathy and motor neuron disease. Prog Brain Res, 101, 295-303.

LATOV N (1995) Pathogenesis and therapy of neuropathies associated with monoclonal gammopathies. Ann Neurol, 37(suppl 1), S32-42. 
LATOV N (2000) Prognosis of neuropathy with monoclonal gammopathy. Muscle Nerve, 23, 150-152.

LEE K-W, INGHIRAMI G, SPATZ L, KNOWLES DM, LATOV N (1991) The Bcells that express anti-MAG antibodies in neuropathy and nonmalignant IgM monoclonal gammopathy belong to the CD5 subpopulation. J Neuroimmunol, 31, 82-88.

LEGER JM, VAUNAIZE J (1990) Polyneuropathy associated with IgM monoclonal gammopathy: a review. Clinical, electrophysiological and pathological features. Nouv Fr Hematol, 32, 303-306.

LEGER JM, HOANG-XUAN K, CHENNOUFI A, SAIDI $H$, BOUCHE $P$, BAUMANN $N$, et al (1992a) Traitement des neuropathies dysimmunitaires par les immunoglobulines polyvalentes humaines intraveineuses. Presse Med, 21, 1533.

LEGER JM, YOUNES-CHENNOUFI AB, ZUBER $M$, BOUCHE $P$, JAUBERTEAU MO, DORMONT D et al (1992b) Frequency of central lesions in polyneuropathy associated with $\operatorname{lgM}$ monoclonal gammopathy: an MRI, neurophysiological and immunochemical study. J Neurol Neurosurg Psychiatry, 55, 112-115.

LEIBOWITZ S, GREGSON NA, KENNEDY M, KAHN SN (1983) IgM paraproteins with immunological specificity for a Schwann cell component and peripheral nerve myelin in patients with polyneuropathy. J Neurol Sci, 59, 153-165.

LEWIS RA, SUMNER AJ, BROWN MJ, ASBURY AK (1982) Multifocal demyelinating neuropathy with persistent conduction block. Neurology, 32, 958-964.

LIN H, BOLLING SF, LINSLEY PS, WEI RQ, GORDON D, THOMPSON CB, et al (1993) Long-term acceptance of major histocompatibility complex mismatched cardiac allografts induced by CTLA4Ig plus donor-specific transfusion. J Exp Med, 178, 1801-1806. 
LINOS A, KYLE RA, O'FALLON WM, KURLAND LT (1981) Incidence and secular trend of multiple myeloma in Olmstead county, Minnesota 1965-77. J Natl Cancer Inst, 66, 17-20.

LOPATE G, PARKS BJ, GOLDSTEIN JM, YEE W-C, FRIESENHAHN G, PESTRONK A (1997a) Polyneuropathies associated with high antisulphatide antibodies: characteristics of patients with and without serum monoclonal proteins. J Neurol Neurosurg Psychiatry, 62, 581585.

LOPATE G, PESTRONK A, KORNBERG AJ, YUE J, CHOKSI R (1997b) IgM anti-sulfatide autoantibodies: Patterns of binding to cerebellum, dorsal root ganglion and peripheral nerve. J Neurol Sci, 151, 189-193.

LOWRY OH, ROSEBOROUGH NJ, FARR AL, RANDALL RJ (1951) Protein measurement with the Folin phenol reagent. J Biol Chem, 93, 265275.

MAISONOBE T, CHASSANDE B, VÉRIN M, JOUNI M, LÉGER JM, BOUCHE $P$ (1996) Chronic dysimmune demyelinating polyneuropathy:a clinical and electrophysiological study of 93 patients. J Neurol Neurosurg Psychiatry, 61, 36-42.

MAMOLI A, NEMNI R, CAMERLINGO M, QUATTRINI A, CASTO L, LORENZETTI I, et al (1992) A clinical, electrophysiological, morphological and immunological study of chronic sensory neuropathy with ataxia and paraesthesia. Acta Neurol Scand, 85, 110-115.

MEIER C, STECK A, VANDEVELDE M (1984a) Immunpathogenese der Polyneuropathien bei Paraproteinämie. Dtsche Med Wschr, 109, 828833.

MEIER C, VANDEVELDE M, STECK A, ZURBRIGGEN A (1984b) Demyelinating polyneuropathy associated with monoclonal IgMparaproteinaemia. Histological, ultrastructural and immunocytochemical studies. J Neurol Sci, 63, 353-367. 
MELMED C, FRAIL D, DUNCAN I, BRAUN P, DANOFF D, FINLAYSON M, et al (1983) Peripheral neuropathy with IgM kappa monoclonal immunoglobulin directed against myelin-associated glycoprotein. Neurology, 33, 1397-1405.

MENDELL JR, SAHENK Z, WHITAKER JN, TRAPP BD, YATES AJ, GRIGGS $R C$, et al (1985) Polyneuropathy and IgM monoclonal gammopathy: studies on the pathogenetic role of anti-myelin-associated glycoprotein antibody. Ann Neurol, 17, 243-254.

MENDELL JR, KOLKIN S, KISSEL JT, WEISS KL, CHAKERES DW, RAMMOHAN KW (1987) Evidence for central nervous system demyelination in chronic inflammatory demyelinating polyradiculoneuropathy. Neurology, 37, 1291-1294.

MIESCHER GC, STECK AJ (1996) Paraproteinaemic neuropathies. Baillière's Clin Neurol, 5, 219-232.

MONACO S, BONETTI B, FERRARI S, MORETTO G, NARDELLI E, TEDESCO F, et al (1990) Complement-mediated demyelination in patients with IgM monoclonal gammopathy and polyneuropathy. $N$ Engl J Med, 322, 649-652.

MOORHOUSE DF, FOX RI, POWELL HC (1992) Immunotactoid-like endoneurial deposits in a patient with monoclonal gammopathy of undetermined significance and neuropathy. Acta Neuropathol(Berl), 84, 484-494.

NARDELLI E, BASSI A, MAZZI G, ANZINI P, RIZZUTO N (1995) Systemic passive transfer studies using lgM monoclonal antibodies to sulfatide. J Neuroimmunol, 63, 29-37.

NEMNI R, FELTRI ML, FAZIO R, QUATTRINI A, LORENZETTI I, CORBO M, et al (1990) Axonal neuropathy with monoclonal IgG kappa that binds to a neurofilament protein. Ann Neurol, 28, 361-364. 
NEMNI R, FAZIO R, QUATTRINI A, LORENZETTI I, MAMOLI D, CANAL N (1993) Antibodies to sulfatide and chondroitin sulfate $C$ in patients with chronic sensory neuropathy. J Neuroimmunol, 43, 79-86.

NOBILE-ORAZIO E, BARBIERI S, BALDINI L, MARMIROLI P, CARPO M, PREMOSELLI S et al (1992) Peripheral neuropathy in monoclonal gammopathy of undetermined significance: prevalence and immunopathogenetic studies. Acta Neurol Scand, 85, 383-390.

NOBILE-ORAZIO E, MEUCCI N, BARBIERI S, CARPO M, SCARLATO G (1993) High-dose intravenous immunoglobulin therapy in multifocal motor neuropathy. Neurology, 43, 537-544.

NOBILE-ORAZIO E, MANFREDINI E, CARPO M, MEUCCI N, MONACO S, FERRARI S, et al (1994) Frequency and clinical correlates of antineural $\operatorname{lgM}$ antibodies in neuropathy associated with $\operatorname{lgM}$ monoclonal gammopathy. Ann Neurol, 36, 416-424.

NORONHA AB, ILYAS A, ANTONICEK H, SCHACHNER M, QUARLES RH (1986) Molecular specificity of L2 monoclonal antibodies that bind to carbohydrate determinants of neural cell adhesion molecules and their resemblance to other monoclonal antibodies recognizing the myelinassociated glycoprotein. Brain Res, 385, 237-244.

NORTON WT, CAMMER W (1984) Isolation and characterization of myelin. In: Morell P, editor. Myelin, 2nd ed. New York: Plenum Press, pp.174178.

NOTERMANS NC, WOKKE JH, LOKHORST HM, FRANSSEN H, VAN DER GRAAF Y, JENNEKENS FG (1994) Polyneuropathy associated with monoclonal gammopathy of undetermined significance. A prospective study of the prognostic value of clinical and laboratory abnormalities. Brain, 117, 1385-1393.

NOTERMANS NC, WOKKE JH, VAN DEN BERG LH, VAN dG, FRANSSEN $H$, TEUNISSEN LL, et al (1996) Chronic idiopathic axonal 
polyneuropathy. Comparison of patients with and without monoclonal gammopathy. Brain, 119, 421-427.

NOTERMANS NC, FRANSSEN H, EURELINGS M, VAN DER GRAAF $Y$, WOKKE JHJ (2000) Diagnostic criteria for demyelinating polyneuropathy associated with monoclonal gammopathy. Muscle Nerve, 23, 73-79.

O'SHANNESSY DJ, WILLISON HJ, INUZUKA T, DOBERSEN MJ, QUARLES $\mathrm{RH}$ (1985) The species distribution of nervous system antigens that react with anti-myelin-associated glycoprotein antibodies. $J$ Neuroimmunol, 9, 255-268.

OBI T, KUSUNOKI S, TAKATSU M, MIZOGUCHI K, NISHIMURA Y (1992) IgM $M$-protein in a patient with sensory-dominant neuropathy binds preferentially to polysialogangliosides. Acta Neurol Scand, 86, 215218.

OGAWA-GOTO K, FUNAMOTO N, ABE T, NAGASHIMA K (1990) Different ceramide compositions of gangliosides between human motor and sensory nerves. J Neurochem, 55, 1486-1493.

OGAWA-GOTO K, FUNAMOTO N, OHTA Y, ABE T, NAGASHIMA K (1992) Myelin gangliosides of human peripheral nervous system: an enrichment of GM1 in the motor nerve myelin isolated from cauda equina. J Neurochem, 59, 1844-1849.

OH SJ (1993a) Physiological factors affecting nerve conduction. In: Oh SJ. Clinical Electromyography. Baltimore: Williams \& Wilkins, p. 310.

OH SJ (1993b) Required tests for specific problems. In: Oh SJ. Clinical Electromyography. Baltimore: Williams \& Wilkins, p. 90.

OHSAWA T, MIYATAKE T, YUKI N (1993) Anti-B-series gangliosiderecognizing autoantibodies in an acute sensory neuropathy patient cause cell death of rat dorsal root ganglion neurons. Neurosci Lett, 157, 167-170. 
OKA H, KUSAKA H, KUSUNOKI S, TSUDA H, KAJI R, IMAI T, et al (1996) IgM M-protein with antibody activity against gangliosides with disialosyl residue in sensory neuropathy binds to sensory neurons. Muscle Nerve, 19, 528-530.

OLNEY RK, PESTRONK A (1992) Prednisone treatment of multifocal predominantly motor neuropathy. Neurology, 42(supplement 3), 178

ÖSBY E, NORING L, HAST R, KJELLIN KG, KNUTSSON E, SIDÉN A (1982) Benign monoclonal gammopathy and peripheral neuropathy. $\mathrm{Br} J$ Haematol, 51, 531-539.

PAPAROUNAS K, O'HANLON GM, O'LEARY CP, ROWAN EG, WILLISON HJ (1999) Anti-ganglioside antibodies can bind peripheral nerve nodes of Ranvier and activate the complement cascade without inducing acute conduction block in vitro. Brain, 122, 807-816.

PARRY GJ (1999) Are multifocal motor neuropathy and Lewis-Sumner syndrome distinct nosologic entities? Muscle Nerve, 22, 557-559.

PATHAK S, ILLAVIA SJ, KHALILI-SHIRAZI A, WEBB HE (1990) Immunoelectron microscopical labelling of a glycolipid in the envelopes of brain cell-derived budding viruses, Semliki Forest, influenza and measles, using a monoclonal antibody directed chiefly against galactocerebroside resulting from Semliki Forest virus infection. $J$ Neurol Sci, 96, 293-302.

PAUL WE (1988) The immune system: introduction. In: R Cecil, editor. Textbook of medicine. Philadelphia: W.B.Saunders, pp. 1932-1937.

PETO R, PIKE MC, ARMITAGE P, BRESLOW NE, COX DR, HOWARD SV et al (1977) Design and analysis of randomized clinical trials requiring prolonged observation of each patient. Br J Cancer, 34, 585-612.

PESTRONK A, CORNBLATH DR, ILYAS AA, BABA H, QUARLES RH, GRIFFIN JW, et al (1988) A treatable multifocal motor neuropathy with antibodies to GM1 ganglioside. Ann Neurol, 24, 73-78. 
PESTRONK A, ADAMS RN, CORNBLATH D, KUNCL RW, DRACHMAN DB, CLAWSON L (1989) Patterns of serum IgM antibodies to GM1 and GD1a gangliosides in amyotrophic lateral sclerosis. Ann Neurol, 25, 98-102.

PESTRONK A, CHAUDHRY V, FELDMAN EL, GRIFFIN JW, CORNBLATH DR, DENYS EH, et al (1990) Lower motor neuron syndromes defined by patterns of weakness, nerve conduction abnormalities, and high titers of antiglycolipid antibodies. Ann Neurol, 27, 316-326.

PESTRONK A, LI F, GRIFFIN J, FELDMAN EL, CORNBLATH D, TROTTER $J$, et al (1991) Polyneuropathy syndromes associated with serum antibodies to sulfatide and myelin-associated glycoprotein. Neurology, 41, 357-362.

PESTRONK A, CHOKSI R, YEE WC, KORNBERG AJ, LOPATE G, TROTTER J (1998) Serum antibodies to heparan sulfate glycosaminoglycans in Guillain-Barré syndrome and other demyelinating polyneuropathies. J Neuroimmunol, 91, 204-209.

POLLARD JD, MCLEOD JG, FEENEY D (1985) Peripheral neuropathy in IgM kappa paraproteinaemia: clinical and ultrastructural studies in two patients. Clin Exp Neurol, 21, 41-54.

POLTORAK M, STECK AJ, SCHACHNER M (1986) Reactivity with neural cell adhesion molecules in sera from patients with demyelinating diseases. Neurosci Lett, 65, 199-203.

POWELL HC, RODRIGUEZ M, HUGHES RAC (1984) Microangiopathy of vasa nervorum in dysglobulinemia neuropathy. Ann Neurol, 15, 386394.

PROVINCIALI L, DI BELLA P, LOGULLO F, VESPRINI L, PASQUINI U, SCARPELLI M (1989) Evidence of central nervous system involvement in chronic demyelinating neuropathies associated with "benign" gammopathies. Riv Neurol, 59, 36-44. 
QIAN G-X, FU SM, SOLANKI DL, RAI KR (1984) Circulating monoclonal IgM proteins in B cell chronic lymphocytic leukemia: their identification, characterization and relationship to membrane IgM. J Immunol, 133, 3396-3400.

QIN Z, GUAN Y (1997) Experimental polyneuropathy produced in guinea-pigs immunized against sulfatide. Neuroreport, 13, 2867-2870.

QUARLES RH, ILYAS AA, WILLISON HJ (1986) Antibodies to glycolipids in demyelinating diseases of the human peripheral nervous system. Chem Phys Lipids, 42, 235-248.

QUARLES RH, WEISS DD (1999) Autoantibodies associated with peripheral neuropathy. Muscle Nerve, 22, 800-822.

QUATTRINI A, NEMNI R, FAZIO R, IANNACCONE S, LORENZETTI I, GRASSI F, et al (1991) Axonal neuropathy in a patient with monoclonal IgM kappa reactive with Schmidt-Lantermann incisures. $J$ Neuroimmunol, 33, 73-79.

QUATTRINI A, CORBO M, DHALIWAL SK, SADIQ SA, LUGARESI A, OLIVEIRA $A$, et al (1992) Anti-sulfatide antibodies in neurological disease: binding to rat dorsal root ganglia neurons. J Neurol Sci, 112, 152-159.

RADL J, SEPERS JM, SKVARIL F, MORELL A, HIJMANS W (1975) Immunoglobulin patterns in humans over 95 years of age. Clin Exp Immunol, 22, 84-90.

RADL J (1985) Benign monoclonal gammopathy. In: Mechanisms in B-cell neoplasia. Edited by F Melchers, M Potter. Berlin: Springer. 221-224.

REBAI T, MHIRI C, HEINE P, CHARFI H, MEYRIGNAC C, GHERARDI R (1989) Focal myelin thickenings in a peripheral neuropathy associated with IgM monoclonal gammopathy. Acta Neuropathol(Ben), 79, 226232. 
RHEE EK, ENGLAND JD, SUMNER AJ (1990) A computer simulation of conduction block: effects produced by actual block versus interphase cancellation. Ann Neurol, 28, 146-156.

ROBERTS M, WILLISON HJ, VINCENT A, NEWSOM-DAVIS J (1995) Multifocal motor neuropathy human sera block distal motor nerve conduction in mice. Ann Neurol, 38, 111-118.

ROPPER AH, GORSON KC (1998) Neuropathies associated with paraproteinemia. N Engl J Med, 338, 1601-1607.

ROTH G, ROHR J, MAGISTRIS MR, OCHSNER F (1986) Motor neuropathy with proximal multifocal persistent conduction block, fasciculations and myokymia. Evolution to tetraplegia. Eur Neurol, 25, 416-423.

RUBIN M, KARPATI G, CARPENTER S (1987) Combined central and peripheral myelinopathy. Neurology, 37, 1287-1290.

RYBERG B (1978) Multiple specificities of antibrain antibodies in multiple sclerosis and chronic myelopathy. J Neurol Sci, 38, 357-382.

SALEUN JP, VICARIOT M, DEROFF P, MORIN JF (1982) Monoclonal gammopathies in the adult population of Finistère, France. $J$ Clin Pathol, 35, 63-68.

SALLOWAY S, MERMEL LA, SEAMANS M, ASPINALL GO, NAM SHIN JE, KURJANCZYK LA, et al (1996) Miller-Fisher syndrome associated with Campylobacter jejuni bearing lipopolysaccharide molecules that mimic human ganglioside GD3. Infect Immun, 64, 2945-2949.

SAPERSTEIN DS, AMATO AA, WOLFE GI, KATZ JS, NATIONS SP, JACKSON CE et al (1999) Multifocal acquired demyelinating sensory and motor neuropathy: the Lewis-Sumner syndrome. Muscle Nerve, 22, 560-566.

SCHEIPERS P, REISER H (1998) Role of the CTLA-4 receptor in T cell activation and immunity. Physiologic function of the CTLA-4 receptor. Immunol Res, 18, 103-115. 
SCHWARTZ RH (1992) Costimulation of T lymphocytes: the role of CD28, CTLA-4, and B7/BB1 in interleukin-2 production and immunotherapy. Cell, 71, 1065-1068.

SCHWARTZ RS (1999) The new immunology-the end of immunosuppressive drug therapy? N Engl J Med, 340, 1754-1756.

SEWELL HF, MATTHEWS JB, GOOCH E, MILLAC P, WILLOX A, STERN $M A$, et al (1981) Autoantibody to nerve tissue in a patient with a peripheral neuropathy and an IgG paraprotein. J Clin Pathol, 34, 1163-1166.

SHERMAN WH, LATOV N, HAYS AP, TAKATSU M, NEMNI R, GALASSI G, et al (1983) Monoclonal $\operatorname{lgM}_{\mathrm{K}}$ antibody precipitating with chondroitin sulphate $C$ from patients with axonal polyneuropathy and epidermolysis. Neurology, 33, 192-201.

SIEVERS J (1971) Basic two-dye stains for epoxy-embedded 0.3-1 $\mu \mathrm{m}$ sections. Stain Technol, 46, 195-199.

SIMMONS Z, BROMBERG MB, FELDMAN EL, BLAIVAS M (1993) Polyneuropathy associated with $\lg A$ monoclonal gammopathy of undetermined significance. Muscle Nerve, 16, 77-83.

SIMMONS Z, ALBERS JW, BROMBERG MB, FELDMAN EL (1995) Longterm follow-up of patients with chronic inflammatory demyelinating polyradiculoneuropathy, without and with monoclonal gammopathy. Brain, 118, 359-368.

SIMOVIC D, GORSON KC, ROPPER AH (1998) Comparison of IgM-MGUS and IgG-MGUS polyneuropathy. Acta Neurol Scand, 97, 194-200.

SMITH IS, KAHN SN, LACEY BW, KING RH, EAMES RA, WHYBREW DJ, et al (1983) Chronic demyelinating neuropathy associated with benign IgM paraproteinaemia. Brain, 106, 169-195. 
SMITH IS, FURNESS P: THOMAS PK (1984) Tremor in peripheral neuropathy. In: LJ Findley, R Capildeo, editors. Movement disorders: Tremor. London: Macmillan, pp. 399-406.

SMITH IS (1994) The natural history of chronic demyelinating neuropathy associated with benign IgM paraproteinaemia. A clinical and neurophysiological study. Brain, 117, 949-957.

SMITH IS (1995) Tremor in peripheral neuropathy. In: Handbook of tremor disorders. Edited by LJ Findley, WC Koller. New York: Marcel Dekker, pp. 443-454.

SPENCER SS, MOENCH JC (1980) Progressive and treatable cerebellar ataxia in macroglobulinemia. Neurology, 30, 536-538.

STECK AJ, MURRAY N, MEIER C, PAGE N, PERRUISSEAU G (1983) Demyelinating neuropathy and monoclonal IgM antibody to myelinassociated glycoprotein. Neurology, 33, 19-23.

STECK AJ, MURRAY N, JUSTAFRE JC, MEIER C, TOYKA KV, HEININGER $K$, et al (1985) Passive transfer studies in demyelinating neuropathy with IgM monoclonal antibodies to myelin-associated glycoprotein. $J$ Neurol Neurosurg Psychiatry, 48, 927-929.

STECK AJ, MURRAY N, DELLAGI K, BROUET JC, SELIGMANN M (1987) Peripheral neuropathy associated with monoclonal IgM autoantibody. Ann Neurol, 22, 764-767.

STEFANSSON K, MARTON LS, ANTEL JP, WOLLMANN RL, ROOS RP, CHEJFAC G, et al (1983) Neuropathy accompanying IgM $\lambda$ monoclonal gammopathy. Acta Neuropathol(Berl), 59, 255-261.

STERNBERGER NH, QUARLES RH, ITOYAMA Y, WEBSTER H (1979) Myelin-associated glycoprotein demonstrated immunocytochemically in myelin and myelin-forming cells of developing rat. Proc Natl Acad Sci USA, 76, 1510-1514. 
SUAREZ GA, KELLY JJ Jr (1993) Polyneuropathy associated with monoclonal gammopathy of undetermined significance: further evidence that IgMMGUS neuropathies are different than IgG-MGUS. Neurology, 43, 1304-1308.

TAKATSU M, HAYS AP, LATOV N, ABRAMS GM, NEMNI R, SHERMAN WH, et al (1985) Immunofluorescence study of patients with neuropathy and IgM M proteins. Ann Neurol, 18, 173-181.

TATUM AH (1993) Experimental paraprotein neuropathy, demyelination by passive transfer of human IgM anti-myelin-associated glycoprotein. Ann Neurol, 33, 502-506.

TETTAMANTI G, RIBONI L (1993) Gangliosides and modulation of the function of neural cells. Adv Lipids Res, 25, 235-267.

THOMAS PK, WALKER RWH, RUDGE P, MORGAN-HUGHES JA, KING RHM, JACOBS JM, et al (1987) Chronic demyelinating peripheral neuropathy associated with multifocal central nervous system demyelination. Brain, 110, 53-76.

THOMAS PK, WILLISON HJ (1994) Paraproteinaemic neuropathy. Bailliere's Clin Neurol, 3, 129-147.

TIVOL EA, BORRIELLO F, SCHWEITZER AN, LYNCH WP, BLUESTONE JA, SHARPE AH (1995) Loss of CTLA-4 leads to massive lymphoproliferation and fatal multiorgan tissue destruction, revealing a critical negative regulatory role of CTLA-4. Immunity, 3, 541-547.

TODA G, IKEDA Y, KASHIWAGI M, IWAMORI M, OKA H (1990) Hepatocyte plasma membrane glycosphingolipid reactive with sera from patients with autoimmune chronic active hepatitis: its identification as sulfatide. Hepatology, 12, 664-670.

TOWBIN H, STAEHELIN T, GORDON J (1979) Electrophoretic transfer of proteins from polyacrylamide gels to nitrocellulose sheets: procedure and some applications. Proc Natl Acad Sci USA, 76, 4350-4354. 
TROJABORG $W$, HAYS AP, VAN DEN BERG L, YOUNGER DS, LATOV $N$ (1995) Motor conduction parameters in neuropathies associated with anti-MAG antibodies and other types of demyelinating and axonal neuropathies. Muscle Nerve, 18, 730-735.

TURNER M (1998) Antibodies and their receptors. In: Riott I, Brostoff J, Male D, editors. Immunology. London: Mosby International, pp.71-82.

VALLAT JM, LEBOUTET MJ, JAUBERTEAU MO, TABARAUD $F$, COURATIER P, AKANI F (1994) Widenings of the myelin lamellae in a typical Guillain-Barré syndrome. Muscle Nerve, 17, 378-380.

VALLAT JM, JAUBERTEAU MO, BORDESSOULE D, YARDIN C, PREUX PM, COURATIER P (1996) Link between peripheral neuropathy and monoclonal dysglobulinemia: a study of 66 cases. J Neurol Sci, 137, 124-130.

VALLDEORIOLA F, GRAUS F, STECK AJ, MUÑOZ E, DE LA FUENTE M, GALLART $T$, et al (1993) Delayed appearance of anti-myelinassociated glycoprotein antibodies in a patient with chronic demyelinating polyneuropathy. Ann Neurol, 34, 394-396.

VAN DEN BERG LH, KINSELLA LJ, CORBO M, YOUNGER D, SADIQ SA, NOBILE-ORAZIO E, et al (1992) Antibodies to MAG and SGPG in neuropathy. Ann Neurol, 32, 251

VAN DEN BERG LH, LANKAMP CLAM, DE JAGER AEJ, NOTERMANS NC, SODAAR P, MARRINK $J$, et al (1993) Anti-sulphatide antibodies in peripheral neuropathy. J Neurol Neurosurg Psychiatry, 56, 1164-1168.

VAN DEN BERG LH, HAYS AP, NOBILE-ORAZIO E, KINSELLA LJ, MANFREDINI E, CORBO M, et al (1996) Anti-MAG and anti-SGPG antibodies in neuropathy. Muscle Nerve, 19, 637-643.

VAN DEN BERGH P, LOGIGIAN EL, KELLY JJ, Jr. (1989) Motor neuropathy with multifocal conduction block. Muscle Nerve, 12, 26-31. 
VAN VLIET HHDM, KAPPERS-KLUNNE MC, VAN DER HEL JWB, ABELS J (1987) Antibodies against glycosphingolipids in sera of patients with idiopathic thrombocytopenic purpura. Br J Haematol, 67, 103-108.

VISSER LH, VAN DER MECHE FG, MEULSTEE J, ROTHBARTH PP, JACOBS BC, SCHMITZ PI, et al (1996) Cytomegalovirus infection and Guillain-Barré syndrome: the clinical, electrophysiologic, and prognostic features. Dutch Guillain-Barré study group. Neurology, 47, 668-673.

VITAL A, VITAL C, JULIEN J, BAQUEY A, STECK AJ (1989) Polyneuropathy associated with IgM monoclonal gammopathy. Immunological and pathological study in 31 patients. Acta Neuropathol(Berl), 79, 160167.

VITAL C, PAUTRIZEL B, LAGUENY A, VITAL A, BERGOUIGNAN FX, DAVID $B$, et al (1985) Hypermyélinisation dans un cas de neuropathie périphérique avec gammapathie monoclonale bénigne a IgM. Rev Neurol(Paris), 141, 729-734.

VITAL C, DUMAS M, LATINVILLE D, DIB M, VITAL A, BRECHENMACHER C (1986) Relapsing inflammatory demyelinating polyneuropathy in a diabetic patient. Acta Neuropathol(Berl), 71, 94-99.

WALSH FS, CRONIN M, KOBLAR S, DOHERTY P, WINER J, LEON A, et al (1991) Association between glycoconjugate antibodies and Campylobacter infection in patients with Guillain-Barré syndrome. $J$ Neuroimmunol, 34, 43-51.

WAXMAN SG (1993) Peripheral nerve abnormalities in multiple sclerosis. Muscle Nerve, 16, 1-5.

WILLIAMS R, HARDING AE (1985) Pupillary light-near dissociation in paraproteinaemic neuropathy. A case report. Neuro-opthalmology., 5, 227-230. 
WILLISON HJ, TRAPP BD, BACHER JD, DALAKAS MC, GRIFFIN JW, QUARLES RH (1988) Demyelination induced by intraneural injection of human anti-myelin-associated glycoprotein antibodies. Muscle Nerve, 11, 1169-1176.

WILLISON HJ, PATERSON G, VEITCH J, INGLIS G, BARNETT SC (1993) Peripheral neuropathy associated with monoclonal IgM anti-Pr2 cold agglutinins. J Neurol Neurosurg Psychiatry, 56, 1178-1183.

WILLISON HJ, O'HANLON GM, PATERSON G, VEITCH J, WILSON G, ROBERTS $M$, et al (1996) A somatically mutated human antiganglioside $\operatorname{lgM}$ antibody that induces experimental neuropathy in mice is encoded by the variable region heavy chain gene, V1-18. $J$ Clin Invest, 97, 1155-1164.

WIRGUIN I, BRIANI C, SUTURKOVA-MILOSEVIC L, FISHER T, DELLALATTA P, CHALIF P, et al (1997) Induction of anti-GM1 ganglioside antibodies by Campylobacter jejuni lipolysaccharides. J Neuroimmunol, 78, 138-142.

YEE WC, HAHN AF, HEARN SA, RUPAR AR (1989) Neuropathy in IgM lambda paraproteinemia. Immunoreactivity to neural proteins and chondroitin sulfate. Acta Neuropathol(Berl), 78, 57-64.

YEUNG KB, THOMAS PK, KING RHM, WADDY H, WILL RG, HUGHES RA, et al (1991) The clinical spectrum of peripheral neuropathies associated with benign monoclonal $\operatorname{lgM}, \lg G$ and $\lg A$ paraproteinaemia. Comparative clinical, immunological and nerve biopsy findings. J Neurol, 238, 383-391.

YOUNES-CHENNOUFI AB, LEGER JM, HAUW JJ, PREUD'HOMME JL, BOUCHE P, AUCOUTURIER P et al (1992) Ganglioside GD1b is the target antigen for a biclonal lgM in a case of sensory-motor axonal polyneuropathy: involvement of $\mathrm{N}$-acetylneuraminic acid in the epitope. Ann Neurol, 32, 18-23. 
YU RK, ARIGA T (1998) The role of glycosphingolipids in neurological disorders. Mechanisms of immune action. Ann N Y Acad Sci, 845, 285-306.

YUKI $\mathrm{N}$, MIYATANI $\mathrm{N}$, SATO S, HIRABAYASHI Y, YAMAZAKI $M$, YOSHIMURA N, et al (1992) Acute relapsing sensory neuropathy associated with IgM antibody against $B-s e r i e s$ gangliosides containing

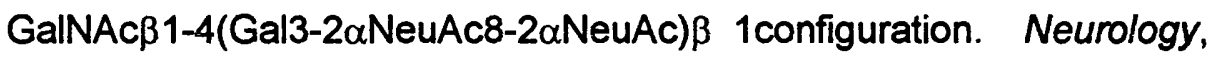
42, 686-689.

YUKI N, TAKI T, INAGAKI $F$, KASAMA T, TAKAHASHI $M$, SAITO $K$, et al (1993) A bacterium lipopolysaccharide that elicits Guillain-Barré syndrome has a GM1 ganglioside-like structure. J Exp Med, 178, $1771-1775$.

YUKI N, TAKI T, TAKAHASHI M, SAITO K, YOSHINO H, TAI T, et al (1994) Molecular mimicry between GQ1b ganglioside and lipopolysaccharides of Campylobacter jejuni isolated from patients with Fisher's syndrome. Ann Neurol, 36, 791-793.

ZALC B, MONGE M, DUPOUEY P, HAUW JJ, BAUMANN NA (1981) Immunohistochemical localization of galactosyl and sulfogalactosyl ceramide in the brain of the 30-day-old mouse. Brain Res, 211, 341354. 
ABSTRACT: The incidence of hematological malignancy in patients with monoclonal gammopathy of undetermined significance (MGUS) has been assessed as $17 \%$ to $25 \%$. To ascertain whether this is true of neuropathy associated with MGUS, a long-term (5-42 years) retrospective clinical and neurophysiological follow-up was conducted in 50 cases (immunoglobulin $M$ [IgM], $n=38 ; \lg G, n=11 ; \lg A, n=1)$. Only three patients developed hematological malignancy. Of 25 survivors with IgM paraproteinemia, 7 had myelin-associated glycoprotein antibodies with typical clinical features. Evoked distal muscle amplitudes were significantly smaller than for the other paraprotein classes. Preferential distal demyelination manifested by relative prolongation of distal motor latency was not apparent in the cases of long duration. Two patients with $\operatorname{lgM}$ antidisialosyl antibodies and cold agglutinating activity had a large fiber neuropathy with intermittent oculofacial involvement. Both responded to intravenous immunoglobulin. Findings in the remaining patients were varied. Recognition of IgM subgroups is important both for prognosis and possible response to treatment.

(c) 2000 John Wiley \& Sons, Inc. Muscle Nerve 23: 164-174, 2000

\section{LONG-TERM CLINICAL AND NEUROPHYSIOLOGICAL FOLLOW-UP OF PATIENTS WITH PERIPHERAL NEUROPATHY ASSOCIATED WITH BENIGN MONOCLONAL GAMMOPATHY}

SAWANTHANA PONSFORD, BSC, FRCP, ${ }^{1}$ HUGH WILLISON, PhD, FRCP, ${ }^{2}$ JEAN VEITCH, ${ }^{2}$ RICHARD MORRIS, PhD, ${ }^{3}$ and P.K. THOMAS, DSC, FRCP4,5

1 Department of Clinical Neurophysiology, Walsgrave Hospital, Clifford Bridge Road, Coventry, CV2 2DX, UK

${ }^{2}$ Department of Neurology, Southern General Hospital, Glasgow, UK

S Department of Primary Care and Population Sciences, Royal Free and University

College Medical School, London, UK

4 Department of Clinical Neurology, Institute of Neurology, London, UK

${ }^{5}$ Department of Clinical Neurosciences, Royal Free and University College Medical

School, London, UK

Accepled 23 August 1999

Peripheral neuropathy associated with monoclonal gammopathy of undetermined significance (MGUS) is an important category of late-onset polyneuropathy. Since the association of benign monoclonal paraproteinemia and peripheral neuropathy was established by Kahn et al. in $1980,{ }^{19}$ subsequent studies have focused mainly on pathogenesis ${ }^{13,14,20,28,29,33,35,47,49,50,58}$ and various specific antibody activities of the parapro-

Abbreviations: anti-S, antisulphatide antibodies; CMAP, compound muscle action potential; Ig, immunoglobulin; MAG, myelin-associated glycopolin; action potential

Key words: immunoglobulins; monoclonal gammopathy; nerve conduction; neuropathy; paraproteinemia

Correspondence to: S. Ponsford; e-mail: sam.ponsford@virgin.net

CCC 0148-639x/00/020164-11

(c) 2000 John Wiley \& Sons, Inc.

teins. ${ }^{1,3-5,7,8,16.18,25,26,36,42,43,59}$ Several subgroups with particular clinical and serological associations have been recognized, predominantly in patients with immunoglobulin M (IgM) paraproteins. These include a chronic demyelinating sensorimotor neuropathy with a paraprotein directed against a sulphated glucuronic acid epitope shared by sulphated glucuronyl paragloboside, myelin-associated glycoprotein (MAG), and myelin protein zero ${ }^{24}$ and multifocal motor neuropathy with paraproteins directed against GM1 ganglioside and related glycoconjugates containing a $\mathrm{Gal}(\beta 1-3)$ GalNAc configuration. ${ }^{41}$ A less constant association has been observed in patients with IgM paraproteinemia and high titers of antisulphatides ( $>4500$, using enzyme-linked immunosorbent assay). ${ }^{30,42,55}$ Few studies have been carried out on the long-term outcome. Although the 
incidence of hematological malignancy was assessed to be as high as $24 \%$ to $26 \%$ in patients with MGUS ascertained hematologically, ${ }^{22,23}$ it is uncertain whether this is true of neuropathy associated with MGUS. A long-term retrospective follow-up on such patients was therefore conducted to assess their outcome. An attempt was also made to identify various subgroups of benign paraproteinemic neuropathy.

\section{MATERIALS AND METHODS}

The patients were identified from the hospital diagnostic index of The National Hospital for Neurology and Neurosurgery, Queen Square, London, and referrals to P.K.T. at the Peripheral Nerve Clinic at that hospital and at the Royal Free Hospital, London, UK. Their case notes were reviewed. All patients included had a peripheral neuropathy with a monoclonal gammopathy, normal bone marrow biopsy (normal cellularity with $<10 \%$ of plasma cells and $<30 \%$ lymphoid cells), normal skeletal $x$-ray survey, and no evidence of Bence-Jones protein in the urine. Other causes of neuropathy were excluded by physical examination and screening tests (complete blood count, erythrocyte sedimentation rate, random blood glucose, urea and electrolytes, liver function tests, thyroid function tests, serum vitamin $B_{12}$, folate concentrations, and chest $x$-ray). Monoclonal gammopathy was detected by agarose gel serum electrophoresis. Immunoelectrophoresis was performed using monospecific sera to IgM, IgG, IgA heavy chains and kappa $(\kappa)$ and lambda $(\lambda)$ light chains. If the results of immunoelectrophoresis were equivocal, immunofixation with monospecific sera was performed. Immunoglobulin (Ig) was quantified by densitometry.

Patients were reviewed clinically and neurophysiologically. Symptoms were scored using the neurological symptom score described by Dyck et al. ${ }^{6}$ Scoring for signs was performed as described in Notermans et al. ${ }^{38}$ in 1994: (1) muscle strength (using the Medical Research Council grading system) in six muscles in each arm and leg (deltoid, biceps, triceps brachii, finger extensors, finger flexors, first dorsal interosseous, iliopsoas, quadriceps femoris, hamstring, anterior tibial, gastrocnemius, peronei) with a total score of 120; (2) tendon reflexes (biceps, triceps, knee, and ankle) were graded as 2 for normal, 1 for reduced, and 0 for absent, the total maximum score being 16; (3) and (4) touch and pin-prick sensation were graded as follows: normal $=4$, distal impairment to wrist $/$ ankle $=3$, distal impairment to half forearm $/$ leg $=2$, distal impairment to elbow and knee $=1$, distal impairment to axilla/groin $=0$, with a total score of 16 for each sensation; (5) vibration (using 128-Hz tuning fork) was graded 4 for perception on middle finger/hallux, 3 for perception on ulnar styloid/medial malleolus but not more distally, 2 for perception to elbow/knee, 1 for perception at clavicle/iliac crest, and 0 for no perception. The maximum scores were 16 ; $(6)$ joint position sense: normal at middle finger $/$ hallux $=2$, diminished $=1$, and absent $=0$, the total scores being 8 . Scoring for clinical disability employed the rating scale given by Hughes et al. ${ }^{15}$ Testing was repeated for paraprotein concentrations and for anti-MAG and antiglycolipid antibodies. Myelin-associated glycoprotein immunoreactivity was identified in Western blots of equine brain myelin delipidated in ether/ethanol (3:2). Protein concentrations were measured by the method of Lowry et al. ${ }^{31}$ Proteins were electrophoretically transferred by the method of Towbin et al. ${ }^{52}$ onto nitrocellulose (Schleicher and Schuell, Keene, $\mathrm{NH})$. The nitrocellulose blots were air dried and stored in the dark prior to immune staining. The assays for antiglycolipid antibody (IgG and IgM activity against GM1, GM2, GM3, GA1, GD1a, GDlb, GT1b, GQ1b, GD3, sulphatides, globoside) and cold agglutinin activity were performed as described by Willison et al. ${ }^{57}$ Antisulphatide and anti-GAl antibodies were considered to be high when the titers exceeded 1/5000. The normal laboratory upper limits for antidisialosyl antibody and the others were $1 / 500$. The patients were then grouped and compared based upon their antibody profiles.

All patients underwent standard nerve conduction studies with supramaximal stimulation employing surface electrodes (Medelec 16893, Medelec, Woking, Surrey, UK) and surface recording electrodes (Medelec 16933). Skin temperature was maintained at or above $32^{\circ} \mathrm{C}$. Electromyography, when performed, was by concentric needle electrodes. Motor nerve conduction studies and F-wave recording were carried out for the median and/or ulnar, common peroneal, and tibial nerves. Sensory nerve action potentials (SNAPs) were examined for the median, ulnar, radial, and sural nerves, the latter two with an antidromic technique. Ring electrodes (Medelec 16639) were used for median and ulnar stimulation over the index and fifth fingers. Criteria for an electrophysiological diagnosis of demyelinating neuropathy were: (1) reduction of motor nerve conduction velocity (MNCV) in two or more nerves to $<70 \%$ of normal $(35 \mathrm{~m} / \mathrm{s}$ in the arms and $29 \mathrm{~m} / \mathrm{s}$ in the legs); (2) prolonged distal latencies in two or more nerves $>150 \%$ (median $>5.4 \mathrm{~ms}$, ulnar $>3.8 \mathrm{~ms}$, common peroneal $>7.2 \mathrm{~ms}$, tibial $>7.6 \mathrm{~ms}$ ); (3) partial conduction block or temporal dispersion in one or more nerves at sites not prone to compres- 
sion (peak-to-peak amplitude criterion, if distalproximal amplitude $>25 \%$ for median, ulnar, and peroneal nerves and $>36 \%$ for tibial nerve; duration criterion if the distal compound muscle potentials (CMAP) total duration $>31 \mathrm{~ms}$, proximal CMAP total duration $>35 \mathrm{~ms}$, proximal-distal difference of duration $>27 \mathrm{~ms}$ for median, ulnar, and peroneal nerves and $>33 \mathrm{~ms}$ for tibial nerve) ${ }^{40}$; (4) absent $\mathrm{F}$ waves or prolonged minimal $\mathrm{F}$-wave latencies in two or more motor nerves of $>150 \%$. Axonal degeneration was considered when there was: (1) CMAP amplitude reduction without temporal dispersion, (2) reduction of MNCV by not more than $30 \%$ of normal, (3) normal MNCV in the presence of denervation potentials on electromyographic examination and (4) absence of SNAPs with the above. Terminal latency index was calculated from terminal distance $(\mathrm{mm})$ divided by conduction velocity $(\mathrm{m} / \mathrm{s}) \times$ terminal latency $(\mathrm{ms})$ and residual latency from terminal latency - distal distance $(\mathrm{mm}) / \mathrm{MNCV}$ in the fore$\operatorname{arm}(\mathrm{m} / \mathrm{s})$.

For patients who died, follow-up information was obtained by telephone contact or by questionnaires to relatives or general practitioners. Three patients had to be traced through the Office for National Statistics. The project was approved by the local ethical committee at the National Hospital for Neurology and Neurosurgery, and all patients gave informed consent.

Survival analysis was performed by constructing Kaplan-Meier survival curves, the differences being compared by a log rank test. Sum score results, when applicable, were compared by using nonparametric tests: Kruskal-Wallis analysis of variance for comparing independent groups and Wilcoxon signed ranks test for comparing paired data. Correlation of distal motor latency and maximal MNCV was undertaken by linear regression and the difference between each group assessed by analysis of variance. Differences were considered significant if $P<0.05$. Only descriptive analyses were performed for groups of fewer than five patients.

\section{RESULTS}

Of the 50 patients studied, 36 were men. The duration of follow-up was 5-42 years, with a mean of 13.7 years. Thirty-eight patients had an IgM paraprotein $(\kappa, 23 ; \lambda, 13$; unclassified, 2), 11 an $\operatorname{IgG}(\kappa, 6 ; \lambda, 5)$ and 1 an IgA $\lambda$. The mean duration of the disease from the onset of neurological symptoms was 13.9 years (range, 5-35 years) for the IgM, 13.3 (range, 5-26 years) for the IgG cases, and 18 years for the IgA case. Mean age at onset of neuropathic symptoms was $56.9,57.4$, and 52 years for the $\operatorname{IgM}, \operatorname{IgG}$, and IgA cases, respectively. The mean paraprotein concentration was 0.54 in IgM and $0.71 \mathrm{~g} / \mathrm{dL}$ in IgG groups, respectively. The paraprotein was not detected on follow-up (after 18 years) in the patient with neuropathy associated with the benign IgA paraprotein.

Outcomo. Fifteen patients died: $13 \mathrm{had}$ an IgM and 2 had an IgG paraproteinemia. Only 3 of the 15 developed hematological malignancy. One developed Waldenström macroglobulinemia from a B cell lymphoma at the age of 65 years, 25 years after the onset of symptoms related to an IgM $\lambda$ demyelinating neuropathy. The diagnosis was prompted by a sudden rise in the level of the paraprotein associated with malaise. The patient died from aspiration pneumonia following an emergency total colectomy for ulcerative colitis at the age of 67 years. The second patient developed refractory anemia with excess blasts at the age of 73 years, 11 years after the onset of symptoms of neuropathy associated with an $\mathrm{IgG \lambda}$ paraprotein. The last patient, who originally had a benign IgM paraproteinemic neuropathy, developed acute myeloid leukemia 17 years after onset of neurological symptoms. All three patients had been on immunosuppressive therapy (azathioprine, melphalan, chlorambucil) for at least 5 years. Four of the 15 developed other malignancies (carcinoma of the colon, liver metastasis from an unknown primary, carcinoma of pancreas, and carcinoma of the pharynx). The first two had had prolonged (11 and 6 years) treatment with cytotoxic drugs (chlorambucil, melphalan); malignancy developed in the year the drug was discontinued in the first and 7 years later in the second. The latter two had received brief courses $(6$ and 10 months) of cytotoxic drugs (cyclophosphamide, azathioprine). One patient with an IgMk paraproteinemic neuropathy has recently been diagnosed and treated for carcinoma of the stomach. The patient has never received immunosuppressive drugs. The remaining patients died from unrelated, nonmalignant illnesses.

Kaplan-Meier log rank tests failed to show significant difference in survival at the $10 \%$ level for the following variables: sex; serum concentration, type, light chain classification or antigenic properties of the paraprotein; degree of disability; or type of neurophysiological abnormality.

Clinical Features. Data for the 35 surviving patients are summarized in Tables 1 and 2. One patient with an IgM paraprotein, who also had a chromosome 17 p11.2 duplication ${ }^{11}$ and a generalized demyelinating sensorimotor neuropathy, was excluded. Case 1 


\begin{tabular}{|c|c|c|c|c|c|c|c|}
\hline $\begin{array}{l}\text { Patient } \\
\text { no. }\end{array}$ & $\begin{array}{l}\text { Sex, age } \\
(y r)\end{array}$ & $\begin{array}{l}\text { Age } \\
\text { at onset } \\
\text { (yr) }\end{array}$ & $\begin{array}{l}\text { Paraprotein } \\
\text { type }\end{array}$ & $\begin{array}{l}\text { Paraprotein } \\
\text { concentration } \\
(g / d L)\end{array}$ & $\begin{array}{l}\text { Antibody } \\
\text { activity }\end{array}$ & $\begin{array}{l}\text { Neuropathy } \\
\text { type }\end{array}$ & $\begin{array}{l}\text { Neurophysiological } \\
\text { findings }\end{array}$ \\
\hline 1 & $F, 82$ & 72 & $\lg M_{\kappa}$ & - & Anti-S & $S>M_{i} \mid>S$ & A \\
\hline 2 & $M, 60$ & 48 & IgMk & 0.9 & Anti-S & $S=M_{i} I=S$ & D + A \\
\hline 3 & $F, 72$ & 57 & $\lg M_{\kappa}$ & 1.0 & Anti-S & $S>M ; I>S$ & $D+A$ \\
\hline 4 & $F, 81$ & 75 & $\lg M \lambda$ & 0.65 & Anti-S & $S>M: I>S$ & $D+A$ \\
\hline 5 & F, 83 & 67 & $\lg M \lambda$ & 0.76 & Anti-S & $S>M_{i} \mid>S$ & $D+A$ \\
\hline 6 & $M, 77$ & 61 & $\lg M \lambda$ & 0.6 & Anti-S & $S>M ; \mid>S$ & $D+A$ \\
\hline 7 & $M, 65$ & 59 & $\lg M_{\kappa}$ & 0.26 & Anti-MAG & $S=M_{i} I>S$ & $D+A$ \\
\hline 8 & M. 70 & 48 & $\lg M_{\kappa}$ & 1.04 & Anti-MAG & $S>M_{i} I>S$ & $D+A$ \\
\hline 9 & M, 72 & 61 & $\lg M_{k}$ & 0.68 & Anti-MAG & $S=M_{i} I>S$ & $D+A$ \\
\hline 10 & $M_{1} 75$ & 53 & $\lg M_{\kappa}$ & 0.84 & Anti-MAG & $S>M_{i} \mid>S$ & $D+A$ \\
\hline 11 & $M, 77$ & 72 & $\operatorname{IgM\lambda }$ & 0.64 & Anti-MAG & $S=M ; I=S$ & $D+A$ \\
\hline 12 & $F, 64$ & 53 & $\lg M \lambda$ & 0.60 & Anti-MAG & $S=M ; 1>S$ & $D+A$ \\
\hline 13 & M, 68 & 52 & $\operatorname{Ig} M \lambda$ & 0.33 & Anti-MAG & $S=M ; 1>S$ & $D+A$ \\
\hline 14 & M, 70 & 60 & $\lg M_{\kappa}$ & 1.11 & Anti-MAG + S & $S=M ; I=S$ & $D+A$ \\
\hline 15 & $M, 58$ & 49 & $\lg M_{\kappa}$ & 0.80 & Anti-MAG + S & $S=M ; I=S$ & $D+A$ \\
\hline 16 & M, 73 & 52 & $\lg M_{\kappa}$ & $t$ & Anti-MAG + S & $S>M ; I>S$ & $D+A$ \\
\hline 17 & $F, 47$ & 40 & $\lg M \lambda$ & 0.45 & Antidisialosyl & $S>M ; l>S$ & $A+D$ (block) \\
\hline 18 & $M, 54$ & 28 & $\lg M \lambda$ & $\neq$ & Antidisialosyl & $S>M ; l>s$ & $A+D$ (block) \\
\hline 19 & $M, 63$ & 58 & $\lg M_{\kappa}$ & 0.41 & - & $S>M_{i} \mid>S$ & $A+D$ (block) \\
\hline 20 & $M, 60$ & 54 & $\lg M_{\kappa}$ & 0.44 & - & $S=M ; I=S$ & $D+A$ \\
\hline 21 & M. 53 & 45 & $\lg M_{\kappa}$ & 0.47 & - & $S>M_{i} I>S$ & $A+D$ (block) \\
\hline 22 & M. 70 & 56 & $\lg M_{\kappa}$ & 0.19 & - & $S>M_{i} I>S$ & A \\
\hline 23 & M. 72 & 54 & IgMk & 0.53 & - & $S>M_{i} \mid>S$ & $A+D$ (block) \\
\hline 24 & $F, 63$ & 49 & $\lg M \lambda$ & $\neq$ & - & $S=M ; I=S$ & $A+D$ (block) \\
\hline
\end{tabular}

-Paraprotein level from the last follow-up.

tBiclonal.

Abbreviations: $S$, sensory; $M$, motor; l, large fiber; $s$, small fiber; $A$ axonal; $D$, demyelinating (predominant change in bold; block, conduction block).

in the IgG/A group could not be adequately assessed for symptoms and signs, as the patient had severe cognitive impairment related to hypertension and small-vessel cerebrovascular disease.

All patients presented with sensory symptoms consisting of numbness and/or paresthesiae. These began either in the legs or hands and legs and pro- gressed proximally. Two cases with IgG paraproteins (18.2\%) presented with sensory disturbances confined to the hands. Five patients with IgM paraproteins $(13.5 \%)$ and 4 patients (36.4\%) with IgG paraproteins had paresthesiae affecting the face. Two of the former had high titers of IgM antisulphatide (anti-S) antibodies, whereas the other two had IgM

\begin{tabular}{|c|c|c|c|c|c|c|}
\hline $\begin{array}{l}\text { Patient } \\
\text { no. }\end{array}$ & $\begin{array}{c}\text { Sex, age } \\
(y r)\end{array}$ & $\begin{array}{c}\text { Age } \\
\text { at onset } \\
(y r)\end{array}$ & $\begin{array}{l}\text { Paraprotein } \\
\text { type }\end{array}$ & $\begin{array}{l}\text { Paraprotein } \\
\text { concentration" } \\
\text { (g/dL) }\end{array}$ & $\begin{array}{l}\text { Neuropathy } \\
\text { type }\end{array}$ & $\begin{array}{c}\text { Neurophysiological } \\
\text { findings }\end{array}$ \\
\hline 1 & $M, 70$ & 60 & $\lg G_{\kappa}$ & 0.64 & $t$ & \\
\hline 2 & $F, 84$ & 70 & $\operatorname{IgG}_{K}$ & 1.02 & $S>M_{i} I>S$ & $D+A$ \\
\hline 3 & F. 75 & 50 & $\lg G_{K}$ & Biclonal & $S=M_{i} \mid>S$ & $D+A$ \\
\hline 4 & $F, 60$ & 44 & $\lg G_{\kappa}$ & 0.35 & $S>M_{i} I>S$ & $A+D$ (block) \\
\hline 5 & M, 79 & 61 & $\lg G_{\kappa}$ & 1.06 & $S>M_{i} \mid>S$ & $A+D$ (block) \\
\hline 6 & $M, 68$ & 63 & $\lg G \lambda$ & 0.42 & $S>M_{i} I=S$ & $D+A$ \\
\hline 7 & $M, 47$ & 39 & $\lg G \lambda$ & ND & $S>M ; I>S$ & $A+D$ (block) \\
\hline 8 & $M, 70$ & 61 & $\lg G \lambda$ & 0.5 & $S>M ; I>S$ & $A+D$ (block) \\
\hline 9 & M, 70 & 56 & $\lg G \lambda$ & 1.10 & $S=M ; I=S$ & $D+A$ \\
\hline 10 & M. 70 & 52 & $\operatorname{Ig} A \lambda$ & ND & $S=M ; I=S$ & $D+A$ \\
\hline
\end{tabular}

-For abbreviations, see footnote to Table 1

tAssessment not possible, see text.

$N D$, not detected. 
antidisialosyl antibodies. The type of antibody activity in the remaining patient with facial sensory symptoms could not be determined, because he had died. Fourteen patients with IgM (38\%), four with IgG $(36 \%)$, and the single patient with an IgA paraprotein had prominent dysesthesiae. These resolved in four of the IgM and two of the IgG cases and in the IgA case, after 3-15 years.

Just over $50 \%$ of patients complained of weakness, but on examination, all patients had at least mild but definite motor involvement. This began or was worse in the lower limbs, apart from three patients with IgG paraproteins whose weakness was more pronounced in the arms. Two of these three patients also had bilateral facial weakness and one had facial sensory symptoms which were responsive to corticosteroid or intravenous Ig treatment. Two patients (nos. 17 and 18) with IgM activity against disialosyl group had intermittent diplopia. Patient no. 18 developed a subacute episode of bilateral facial weakness, ophthalmoplegia, bulbar palsy, global weakness, and respiratory insufficiency necessitating assisted ventilation after an elective hip replacement. The patient recovered to the preoperative state with intravenous Ig therapy. The patient received no treatment before or after the recent acute episode and deteriorated very slowly from walking with crutches to requiring a wheelchair over 26 years. Case no. 17 also experienced a considerable improvement after intravenous Ig therapy from walking with a cane to being able to walk unaided. The effect lasted for only 4-5 weeks.

Tremor was the presenting feature only in patients with IgM paraproteinemia $(50 \%)$ but at the final follow-up examination, 32 of $36(88 \%)$ with IgM and 9 of $11(82 \%)$ with IgG/A paraproteinemia had tremor, which was incapacitating in some. Pseudoathetosis was observed in patients with IgM paraproteinemia ( 8 of $37,21.6 \%$ ): 1 had a high IgM anti-S antibody titer, 3 had anti-MAG activity, 1 had antidisialosyl antibodies, and 2 had unidentified antibody activity. The other patient died. Four of these patients had very slow maximal MNCV: 2.7, 5, 9, 11 $\mathrm{m} / \mathrm{s}$; the first three values were the lowest in the whole series. For the remaining patients, MNCV was between 20 and $25 \mathrm{~m} / \mathrm{s}$. All had prominent largefiber deficits on clinical examination.

Eight patients $(21.6 \%)$ with IgM and two with $\mathrm{IgG} / \mathrm{A}(18.2 \%)$ paraproteins were found to have peripheral nerve thickening other than at the common entrapment sites.

The polyneuropathy had a slowly progressive course with the exception of 3 cases in the IgM $(8.1 \%)$ and 7 in the $\mathrm{IgG} / \mathrm{A}(53 \%)$ group, who had a relapsing/remitting course. Two of the IgM cases had antidisialosyl antibodies; the third IgM case and one of the IgG/A had died. Of the remaining patients, the relapsing/remitting course occurred spontaneously in 3 patients (2 with antidisialosyl, 1 IgA). For IgG case no. 7, relapse occurred with viral infection. The milder relapses took $6-8$ weeks to recover without treatment. The rest occurred either with withdrawal or reduction of steroid therapy.

Comparisons of sum scores of symptoms, signs, and disability between the first and last visits are summarized in Table 3 and in Figure 1. There was a significant difference in the sum scores between the patients with IgM and IgG/A in motor deficits, tendon reflexes, all sensory tests, and disability when the patients were first seen. Similar differences were observed at the final examination apart from motor deficits. Significant change in the sum scores of the same group of patients between the first and last visits was seen only in the IgM group on tendon reflexes $(P=0.001)$, joint position sense $(P=0.001)$, and disability $(P=0.001)$. Improvement in motor, tendon reflex, and disability scores was observed only in the IgG/A group and was seen in 6 of 8 $(75 \%)$ IgG cases. This was associated with steroid therapy in 5 cases. There was no significant difference between the three IgM subgroups-anti-S, antiMAG, and unidentified antibodies-at the time of the initial and final examinations (Fig. 1). More parameters were observed to have a significant deterioration between the two visits in the anti-MAG group.

Laboratory Findings. Among the 24 patients with IgM paraproteins, high titers of anti-S antibodies alone were found in 6 , and 7 had anti-MAG activity alone; 3 had combined anti-MAG and high anti-S activity, and 2 had antidisialosyl activity. The remainder of the patients in the IgM and those in the $\mathrm{IgG} / \mathrm{A}$ groups did not have identifiable antibody activity. The cerebrospinal fluid protein concentration was elevated in 18 of 21 of $\operatorname{IgM}$ cases $(0.6-3.0 \mathrm{~g} / \mathrm{L}$; mean, 1.06) in which this was examined and in 7 out of 7 of the IgG/A cases $(0.7-4.8 \mathrm{~g} / \mathrm{L}$; mean, 1.77$)$. Only $\mathrm{I}$ case showed a slight pleocytosis $(6$ lymphocytes $/ \mathrm{mm}^{3}$ [case no. 16]).

The paraprotein was detected in the blood only on repeating the test approximately 1 year after the first examination in three cases, but immunofixation had not been carried out on the first occasion. ${ }^{17.54}$ Four patients (IgM case nos. 16, 18, and 24 and IgG case no. 3) initially with monoclonal paraproteins had biclonal gammopathy (IgM case no. 16 and IgG case no. 3) and triclonal gammopathy at their last follow-up. Of the 3 IgM cases, the bands were $2 \operatorname{IgMK}$ 


\begin{tabular}{|c|c|c|c|c|}
\hline & \multicolumn{2}{|c|}{$\lg M(n=24)$} & \multicolumn{2}{|c|}{$\lg G / A(n=9)$} \\
\hline & $\begin{array}{c}\text { First } \\
\text { examination }\end{array}$ & $\begin{array}{c}\text { Last } \\
\text { examination }\end{array}$ & $\begin{array}{c}\text { First } \\
\text { examination }\end{array}$ & $\begin{array}{l}\text { Last } \\
\text { examination }\end{array}$ \\
\hline Symptoms & $\begin{array}{l}66 ; 2.75 \pm 0.99 \\
(1-5)\end{array}$ & $\begin{array}{l}92 ; 3.82 \pm 0.87 \\
(1-5)\end{array}$ & $\begin{array}{c}27 ; 3.0 \pm 1.5 \\
(1-5)\end{array}$ & $\begin{array}{c}26 ; 2.89 \pm 1.36 \\
(0-4)\end{array}$ \\
\hline Muscle strength & $\begin{array}{c}2717: 113.2 \pm 7.82 \\
(91-120), P^{*}\end{array}$ & $\begin{array}{l}2335 ; 97.29 \pm 16.39 \\
(62-120)\end{array}$ & $\begin{array}{c}934: \\
103.78 \pm 10.97 \\
(86-120)\end{array}$ & $\begin{array}{c}952 ; \\
\quad 105.78 \pm 18.23 \\
(64-120)\end{array}$ \\
\hline Tendon reflexes & $\begin{array}{c}233 ; 9.71 \pm 6.89 \\
(0-20), P^{*}\end{array}$ & $\begin{array}{c}114 ; 4.75 \pm 6.12 \\
(0-16), P^{*}\end{array}$ & $\begin{array}{c}60 ; 6.67 \pm 6.6 \\
(0-16)\end{array}$ & $\begin{array}{c}71: 7.89 \pm 6.75 \\
(0-16)\end{array}$ \\
\hline Light touch & $\begin{array}{c}280 ; 11.67 \pm 3.9 \\
(3-16), P^{*}\end{array}$ & $\begin{array}{r}216: 9 \pm 3.28 \\
(0-14), P^{\ddagger}\end{array}$ & 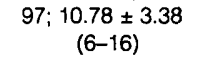 & $\begin{array}{c}96 ; 10.67 \pm 5 \\
(2-16)\end{array}$ \\
\hline Pin prick & $\begin{array}{c}289 ; 12.04 \pm 3.45 \\
(4-16), P^{ \pm}\end{array}$ & $\begin{array}{r}215 ; 9 \pm 2.88 \\
(0-14), P^{\ddagger}\end{array}$ & $\begin{array}{c}100 ; 11.11 \pm 3.76 \\
(6-16)\end{array}$ & $\begin{array}{c}94 ; 10.44 \pm 4.33 \\
(2-16)\end{array}$ \\
\hline Vibration & $\begin{array}{c}295 ; 12.29 \pm 3 \\
(2-16), P^{\ddagger}\end{array}$ & $\begin{array}{c}161 ; 6.71 \pm 4.18 \\
(0-13), P^{*}\end{array}$ & $\begin{array}{c}98 ; 10.89 \pm 3.48 \\
(4-14)\end{array}$ & $\begin{array}{c}77 ; 8.56 \pm 5.08 \\
(0-16)\end{array}$ \\
\hline Joint position & $\begin{array}{c}114 ; 4.75 \pm 2.63 \\
(0-8), P^{\neq}\end{array}$ & $\begin{array}{l}68 ; 2.83 \pm 2.57 \\
(0-8), P^{\ddagger}\end{array}$ & $\begin{array}{c}49 ; 5.44 \pm 2.3 \\
(2-8)\end{array}$ & $\begin{array}{c}46 ; 5.11 \pm 2.2 \\
(2-8)\end{array}$ \\
\hline Disability & $\begin{array}{l}45 ; 1.88 \pm 0.95 \\
(1-3), P^{\ddagger}\end{array}$ & $\begin{array}{l}68 ; 2.83 \pm 0.87 \\
(1-4), P^{ \pm}\end{array}$ & $\begin{array}{c}19 ; 2.11 \pm 0.78 \\
(1-3)\end{array}$ & $\begin{array}{c}21: 2.33 \pm 0.87 \\
(1-3)\end{array}$ \\
\hline
\end{tabular}

$P$, significant difference in values between groups of patients either at time of initial or final examination.

$P P<0.01$.

bands in case no. 16, $1 \mathrm{IgMK}$ and $2 \mathrm{IgM} \lambda$ in case no. 18 , and $2 \operatorname{Ig} M \lambda$ and $1 \operatorname{IgA} \lambda$ in case no. 24. Case no. 3 , initially with a monoclonal IgGk band, had IgGK and IgMк bands. No paraprotein band was detected at the last follow-up in two patients, one with an IgG (case no. 7) and the other with an IgA paraprotein (case no. 10), the latter with clinical and electrophysiological improvement. No patient showed suppression of other Igs. The nerve biopsy findings in these patients have been reported by Yeung et al in $1991 .^{60}$

Neurophysiological Findings. All patients had a sensorimotor neuropathy at their last follow-up (Tables 1, 2, and 4). The mean time intervals between the initial and the last nerve conduction studies were 8.9 years for $\operatorname{IgM}(9,10,8.7,9.5$, and 7.6 years for anti-S, anti-MAG, anti-MAG $+S$, antidisialosyl, and unidentified antibody subgroups, respectively) and 10.4 years for IgG/A. Two patients (case nos. 1 and 22) in the IgM group had an axonal neuropathy, whereas the rest had a predominantly demyelinating neuropathy. Patients with anti-MAG IgM activity had a significantly prolonged ulnar residual latency and a low-amplitude distal CMAP in comparison with the other IgM subgroups and the IgG/A cases. None had conduction block. There was a significant relative prolongation of distal motor latency in comparison with MNCV in the forearm for the IgM cases with anti-S and with anti-MAG with and without anti-S activity when the patients were first

seen. This correlation was no longer evident at the final follow-up (Table 5). There was little correlation between the terminal latency index and the type of paraprotein for the median nerve at the follow-up examination (Fig. 2A), although a tendency for a relative distal prolongation was still evident in the ulnar nerve for the anti-MAG and anti-S patients (Fig. 2B). Six of the patients with unidentified IgM antibody activity and four in the IgG/A group had asymmetric demyelination with evidence of conduction block. Improvement of sensory nerve conduction velocity (reversal from unrecordable to recordable SNAPs) and MNCV (either reversal from unrecordable to recordable MNCV or an increase in MNCV $>8 \mathrm{~m} / \mathrm{s}^{39}$ ) was seen in three patients in the IgG/A group.

\section{Discussion}

The incidence of hematological malignancy in our patients with paraproteinemic neuropathy is low in comparison with the previously reported rate of $17.4 \%$ of multiple myeloma on 24-38-year follow-up in patients with MGUS. ${ }^{23}$ The diagnosis in the present series was made $11-25$ years after the detection of the paraprotein, and in the only case with available details, this was preceded by a sudden rise in the paraprotein concentration on routine checkup. The diagnosis should be considered even with a stable paraprotein level when there is a rapid clinical deterioration. ${ }^{38}$ A low incidence of hematological malignancy has also been noted on shorter follow-up stud- 

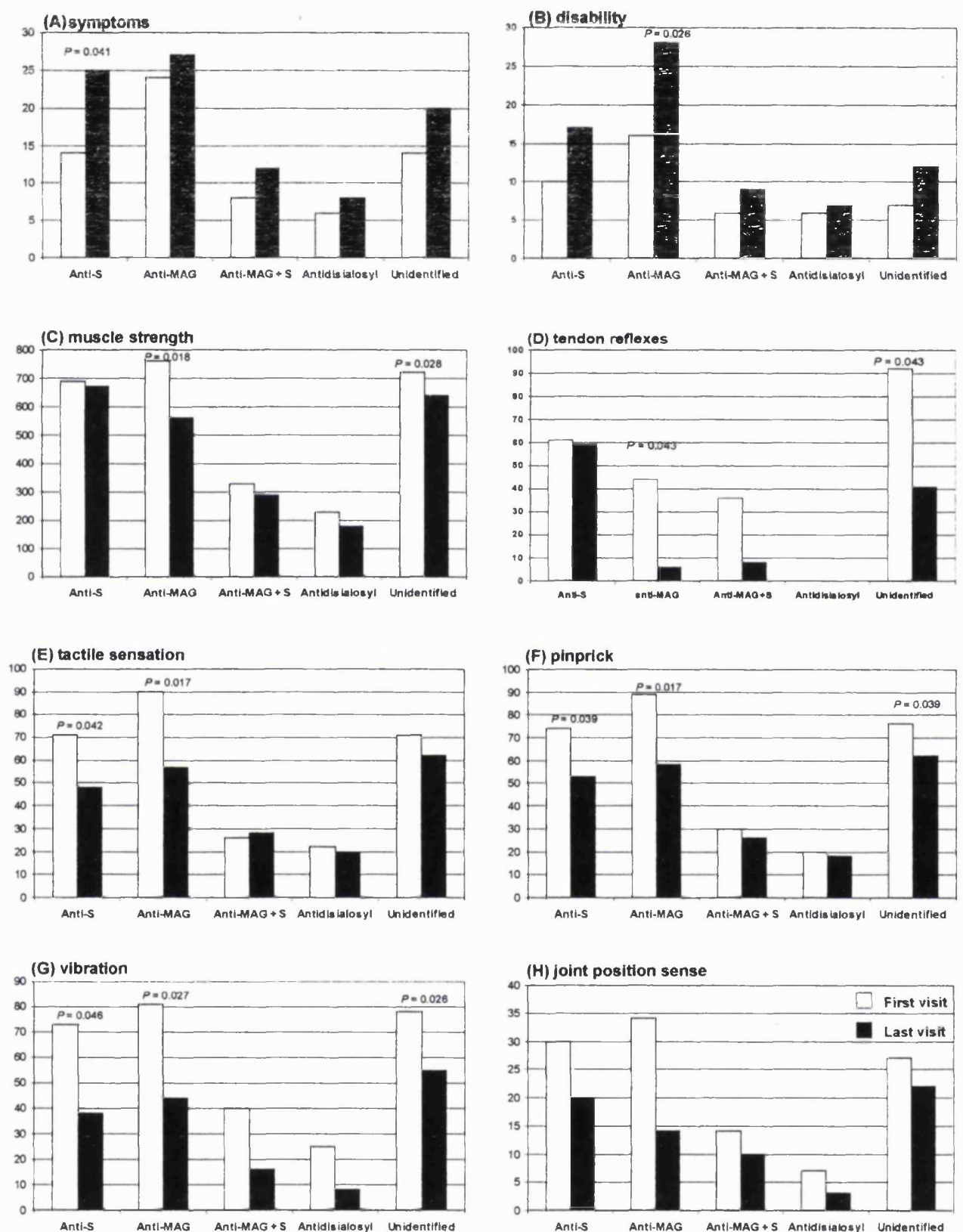

FIGURE 1. Comparison of scores between the first and last visits for each IgM subgroups. $P$ : significant difference in values for the same group of patients between the first and last visits. 


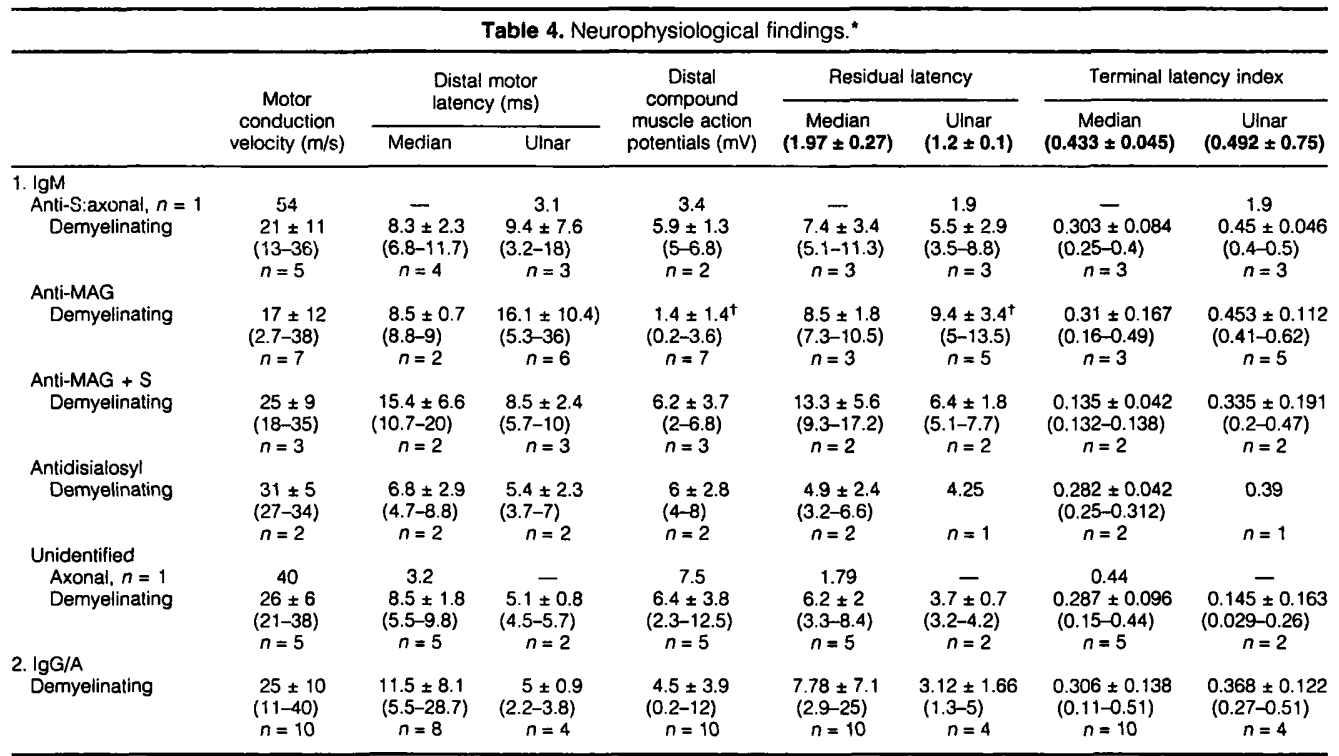

"When applicable, mean \pm standard deviation and range in parentheses; normal values in bold; MNCV and distal CMAP values are for either median

or uignificant difierence in values between groups of patients $P<0.05$

ies. $^{\text {7,38,46 }}$ Nonhematological malignancy, especially of the gastrointestinal system, constituted a common cause of death, but the latter may reflect the high incidence of colorectal and pancreatic malignancy in a population of this age. Precipitation by the administration of cytotoxic drugs also has to be considered. As hematological malignancy usually occurs late, regular follow-up of these patients should be carried out for an indefinite period.

Immunoglobulin $\mathrm{M}$ paraproteinemic neuropathy as a whole consists of a number of heterogeneous subgroups. Benign monoclonal IgM paraproteinemic neuropathy with anti-MAG activity forms a relatively uniform clinical and neurophysiological entity. Our experience confirms late onset, male predominance, and a progressive distal symmetric sensorimo- tor polyneuropathy initially with predominantly large-fiber sensory involvement (in particular, vibration sense). Postural tremor and pseudoathetosis are commonly found at an early stage of the disease. ${ }^{46,47}$ Upper limb postural tremor is frequently evident in the face of normal joint position sense. Studies by positron emission tomography ${ }^{2}$ have suggested that it is generated by the cerebellum and probably other related circuits in response to an abnormal sensory input. Imaging of the cerebellum has been consistently normal. Progression of the neuropathy was slow but steady in the present series. Despite the relatively stable MNCV after 5 years, ${ }^{46}$ our data on serial electromyographic examination and distal CMAP amplitude recordings showed progressive change indicating ongoing axonal degeneration.

\begin{tabular}{|c|c|c|c|c|c|}
\hline \multirow[b]{2}{*}{ Paraprotein reactivity } & \multirow[b]{2}{*}{ No. of patients } & \multicolumn{2}{|c|}{ First assessment } & \multicolumn{2}{|c|}{ Final assessment } \\
\hline & & Slope coefficient & $P$ value & Slope coefficient & $P$ value \\
\hline \multicolumn{6}{|l|}{$\lg M$} \\
\hline Anti-S & 5 & -0.40 & 0.04 & -0.36 & 0.07 \\
\hline Anti-MAG & 7 & -0.58 & 0.03 & -0.53 & 0.10 \\
\hline Unidentified & 5 & -0.37 & 0.06 & 0.05 & 0.74 \\
\hline $\lg G / A$ & 10 & -0.11 & 0.54 & -0.36 & 0.25 \\
\hline
\end{tabular}



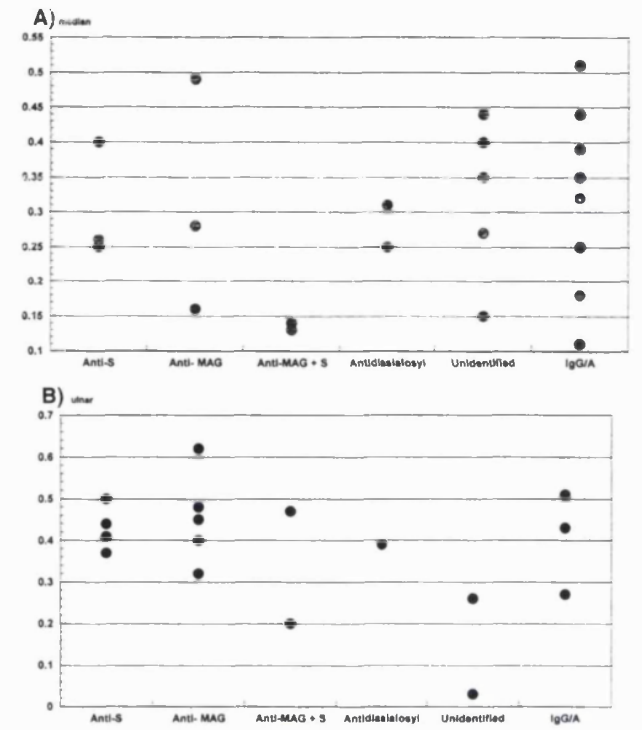

FIGURE 2. Comparison of terminal latency index between various subgroups of $\lg M$ and $\lg \mathrm{G} / \mathrm{A}$ paraproteins.

The neuropathy extended more proximally both from clinical and electrophysiological observation. Conduction block was not a feature in any of our IgM cases with anti-MAG activity, and none had facial or bulbar involvement or a relapsing/remitting course. The three patients with anti-MAG and high anti-S activity could not be distinguished from those with only anti-MAG activity apart from relative preservation of distal CMAP amplitude. In general, the deficits in the anti-MAG IgM cases were more severe and more progressive than in the other groups. Our experience therefore supports the previous findings of cases with anti-MAG activity as a distinctive subgroup of IgM paraproteinemic neuropathy, ${ }^{12,26,27,32,34,42,53,56}$ even though this has not been the experience of others. ${ }^{10,37,38,45,48}$ The explanation of the lower incidence of anti-MAG activity at $28 \%$ in comparison with the $50 \%$ to $65 \%$ found in other series is uncertain.

Kaku et al. ${ }^{21}$ drew attention to a relative accentuation of distal motor latencies in patients with IgM paraproteinemic neuropathy with anti-MAG activicy and antibodies to sulphated glucuronyl paragloboside indicating a greater prominence of demyelination peripherally. This was confirmed at the time of the initial electrophysiological studies in our patients but was no longer evident after prolonged follow-up times, suggesting a centripetal progression of the de- myelination or secondary axonal degeneration leading to normalization of the terminal latency index from loss of severely affected fibers. The latter explanation is supported by the significantly reduced mean distal CMAP in the anti-MAG group.

Of considerable interest were the two patients with antibody activity against disialosyl groups. Both shared a similar clinical phenotype consisting of fuc tuating oculofacial and/or bulbar symptoms on a background of a slowly progressive, ataxic, largefiber sensorimotor neuropathy. The paraprotein was $\operatorname{IgM} \lambda$, which reacted with disialosyl groups on gan gliosides as well as having cold agglutinin activity with anti-Pr2. These cases were similar to those reported in the literature (reviewed by Willison et al. in $1993^{57}$ ).

Six patients who had IgM paraproteinemia with high anti-S titers had a sensorimotor neuropathy with prominent large-fiber involvement beginning in the lower limbs. One had an axonal neuropathy, whereas the remainder had a predominantly demyelinating neuropathy. Sensory nerve action potentials were usually present when the patients were first seen but became absent within the first year. Prominent pain or dysesthesia was found in three patients, including the one with axonal neuropathy. Three of these patients had an IgMk paraprotein. Immunoglobulin M paraproteinemic neuropathy with high anti-S titers therefore do not conform to a single clinical phenotype. ${ }^{30,42,55}$

None of our patients with IgG/A paraproteinemic neuropathy had a pure axonal neuropathy. It was usually mixed axonal and demvelinating with a prominent demyelinating component including conduction block $(4 / 10 \mathrm{IgG} / \mathrm{A})$. The absence of instances of axonal neuropathy, which are not uncommon in this disorder, ${ }^{9.38}$ may relate to the small number of cases. These cases also had high incidence of facial involvement. More than $50 \%$ had a relapsing/ remitting but progressive course and occasionally showed clinical and electrophysiological improvement. On the whole, they were less disabled than those with IgM paraproteins. Some of the patients were similar to those reported by Simmons et al. ${ }^{44}$ The features in the IgG/A group suggest that they behave like chronic inflammatory demyelinating polyneuropathy without associated paraprotein emia. ${ }^{51}$ No patient in our study was found to have IgM anti-GMl activity and/or clinical manifestations of multifocal motor neuropathy or a lower motor neuron disease.

In conclusion, the risk of development of hematological malignancy exists but is much lower than in cases ascertained by the presence of a paraprotein in 
the absence of neuropathy. The possibility exists that malignant change was precipitated by immunosuppressive therapy. Cases of paraproteinemic neuropathy with anti-MAG and with antidisialosyl antibody activity have consistent clinical and serological associations, whereas a less consistent clinical pattern is evident in IgM cases with high anti-S titers. Patients with IgM paraproteins with unidentifiable antibody activity and those with $\mathrm{IgG} / \mathrm{A}$ paraproteins are clinically and electrophysiologically heterogenous.

Presented in part at the XI International Congress of EMG and Clinical Neurophysiology, Prague, September 1999. The authors thank Mr. John Muddle, Dr. John Scott, and the Office for National Statistics for their assistance. We are indebted to all the physicians and patients who kindly participated in the project.

\section{REFERENCES}

1. Ariga T, Kohriyarna T, Freddo L, Latov N, Saito M, Kon K, Ando S, Suzuki M, Hemling ME, Rinehart KL Jr, Kusunoki S, Yu RK. Characterization of sulfated glucuronic acid containing glycolipids reacting with IgM M-proteins in patients with neuropathy. J Biol Chem 1987;262:848-853.

2. Bain PG, Britton TC, Jenkins IH, Thompson PD, Rothwell JC, Thomas PK, Brooks DJ, Marsden CD. Tremor associated with benign IgM paraproteinaemic neuropathy. Brain 1996;119: 789-799.

3. Bollensen E, Steck AJ, Schachner M. Reactivity with the peripheral myelin glycoprotein $P_{0}$ in serum from patients with monoclonal ogy 1988;38:1266-1270.

4. Braun PE, Frail DE, Latov N. Myelin-associated glycoprotein is the antigen for a monoclonal IgM in polyneuropathy. J Neuthe antigen for a monoclonal
rochem 1982;39:1261-1265.

5. Chou DKH, llyas AA, Evans JE, Costello C, Quarles RH, Jungalwala FB. Structure of sulfated glucuronyl glycolipids in the nervous system reacting with HNK-1 antibody and some IgM paraproteins in neuropathy. J Biol Chem 1986;261: 11717-11725.

6. Dyck PJ, Sherman WR, Hallcher LM, Service JF, O'Brien PC, Grina LA, Palumbo PJ, Swanson CJ. Human diabetic endoneurial sorbitol, fructose, and myo-inositol related to sural nerve morphometry. Ann Neurol 1980;8:590-596.

7. Ellie E, Vital A, Steck A, Boiron JM, Vital C, Julien J. Neuropathy associated with "benign" anti-myelin-associated glycoprotein IgM gammopathy: clinical, immunological, neurophysiological pathological findings and response to treatment physiological pathological findings and

8. Freddo L, Yu RK, Latov N, Donofrio PD, Hays AP, Greenberg HS, Albers JW, Allessi AG, Keren D. Gangliosides GMI and $\mathrm{GD} l \mathrm{~b}$ are antigens for IgM M-protein in a patient with motor neurone disease. Neurology 1986;36:454-458.

9. Gorson KC, Ropper AH. Axonal neuropathy associated with monoclonal gammopathy of undetermined significance. J Neurol Neurosurg Psychiatry 1997;63:163-168.

10. Gosselin S, Kyle RA, Dyck PJ. Neuropathy associated with monoclonal gammopathies of undetermined significance. Ann Neurol 1991;30:54-61.

11. Gregory R, Thomas PK, King RHM, Hallam PL, Malcolm S, Gregory R, Thomas PK, King RHM, Hallam PL, Malcolm S,
Hughes RA, Harding AE. Coexistence of hereditary motor and sensory neuropathy type la and IgM paraproteinemic neuropathy. Ann Neurol 1993;

12. Hafler DA, Johnson D, Kelly JJ, Panitch H, Kyle R, Weiner HL Monoclonal gammopathy and neuropathy: myelin-associated glycoprotien reactivity and clinical characteristics. Neurology
$1986 ; 36: 75-78$.
13. Hays AP, I. : v N, Takatsu M, Sherman WH. Experimental demyelination of nerve induced by serum of patients with neuropathy and an anti-MAG IgM M-protein. Neurology 1987;37:242-256.

14. Hays AP, Lee SS, Latov N. Immune reactive C3d on the surface of myelin sheaths in neuropathy. J Neuroimmunol 1988; $18: 231-244$

15. Hughes RAC, Newsom-Davis JM, Perkin GD, Pierce JM. Controlled trial of prednisolone in acute polyneuropathy. Lancet 1978;2:750-753

16. Ilyas AA, Quarles RH, MacIntosh TD, Dobersen MJ, Trapp $B D$, Dalakas MC, Brady RO. IgM in a human neuropathy related to paraproteinemia binds to a carbohydrate determinant in the myelin-associated glycoprotein and to a ganglioside. Proc Natl Acad Sci USA 1984;81:1225-1229.

17. Julien J, Vital C, Vallat JM, Lagueny A, Ferrer X, Leboutet MJ. Chronic demyelinating neuropathy with IgM-producing lymphocytes in peripheral nerve and delayed appearance of "benign" monoclonal gammopathy. Neurology 1984;34: 1387-1389.

18. Kabat EA, Liao J, Sherman WH, Osserman EF. Immunochemical characterization of the specificities of two human monoclonal IgMs reacting with chondroitin sulfates. Carbohydr Res 1984;130:289-297.

19. Kahn SN, Riches PG, Kohn J. Paraproteinaemia in neurological disease: incidence, associations, and classification of monoclonal immunoglobulins. J Clin Pathol 1980;33: 617-621.

20. Kahn SN, Smith IS, Eames RA, Thomas PK, Lacey BW. IgM paraproteinemia and autoimmune peripheral neuropathy. $\mathrm{N}$ Engl J Med 1981;304:1430-1431.

21. Kaku DA, England JD, Sumner AJ. Distal accentuation of conduction slowing in polyneuropathy associated with antibodies to myelin-associated glycoprotein and sulphated glucuronyl paragloboside. Brain 1994;117:941-947.

22. Kyle RA. "Benign" monoclonal gammopathy-after 20-35 years of follow-up. Mayo Clin Proc 1993;68:26-36.

23. Kyle RA. Monoclonal gammopathy of undetermined significance (MGUS). Baillière's Clinical Haematology 1995;8: 761-781.

24. Latov N. Antibodies to glycoconjugates in neurological disease. Clin Aspects Autoimmun 1990;4:18-29.

25. Latov N. Evaluation and treatment of patients with neuropathy and monoclonal gammopathy. Semin Neurol 1994;14: 118-122.

26. Latov N. Pathogenesis and therapy of neuropathies associated with monoclonal gammopathies. Ann Neurol 1995;37(suppl 1):S32-S42.

27. Latov $N$, Hays AP, Sherman WH. Peripheral neuropathy and anti-MAG antibodies. CRC Crit Rev Neurobiology 1988;3: $301-332$.

28. Latov N, Sherman WH, Nemni R, Galassi G, Shyong JS, Penn AS, Chess L, Olarte MR, Rowland LP, Osserman EF. Plasma cell dyscrasia and peripheral neuropathy with a monoclonal antibody to peripheral nerve myelin. N Engl J Med 1980;303: antibody

29. Leibowitz S, Gregson NA, Kennedy M, Kahn SN. IgM paraproteins with immunological specificity for a Schwann cell component and peripheral nerve myelin in patients with polyneuropathy. J Neurol Sci 1983;59:153-165.

30. Lopate G, Parks BJ, Goldstein JM, Yee WC, Friesenhahn G, Pestronk A. Polyneuropathies associated with high titre antisulphatide antibodies: characteristics of patients with and without serum monoclonal proteins. J Neurol Neurosurg Psychiatry 1997;62:581-585.

31. Lowry OH, Rosebrough NJ, Farr AL, Randall RJ. Protein measurement with the Folin phenol reagent. J Biol Chem 1951; 93:265-275.

32. Maisonobe $T$, Chassande $B$, Vérin $M$, Jouni $M$, Léger JM, Bouche P. Chronic dysimmune demyelinating polyneuropa- 
thy: a clinical and electrophysiological study of 93 patients. J Neurol Neurosurg Psychiatry 1996;61:36-42.

33. Meier C, Vandevelde M, Steck A, Zurbriggen A. Demyelinating polyneuropathy associated with monoclonal IgMparaproteinaemia. Histological, ultrastructural and immunocytochemical studies. J Neurol Sci 1984;63:353-367.

34. Mendell JR, Sahenk Z, Whitaker JN, Trapp BD, Yates AJ, Griggs RC, Quarles RH. Polyneuropathy and IgM monoclonal gammopathy: studies on the pathogenetic role of anti-my gammopathy: studies on the pathogenetic role of anti-my elin-associate

35. Monaco S, Bonetti B, Ferrari S, Moretto G, Nardelli E, Te desco F, Mollnes TE, Nobile-Orazio E, Manfredini E, Bonazzi $\mathrm{L}$, Rizzuto N. Complement-mediated demyelination in patients with IgM monoclonal gammopathy and polyneuropathy. N Engl J Med 1990;322:649-652.

36. Nobile-Orazio E, Manfredini E, Carpo M, Meucci N, Monaco S, Ferrari S, Bonetti B, Cavaletti G, Gemignani F, Durelli L, Sgari M, Scarlato G. Frequency and clinical correlates of anti-neural IgM antibodies in neuropachy associated with IgM anti-neural IgM antibodies in neuropachy associated with

37. Notermans NC, Lokhorst HM, Franssen H, van der Graaf Y, Teunissen LL, Jennekens FGI, van den Berg LH, Wokke JH. Intermittent cyclophosphamide and prednisone treatment of polyneuropathy associated with monoclonal gammopathy of undetermined significance. Neurology 1996;47:1227-1233.

38. Notermans NC, Wokke JHJ, Lokhorst HM, Franssen H, van der Graf Y, Jennekens FGI. Polyneuropathy associated with monoclonal gammopathy of undetermined significance. A prospective study of the prognostic value of clinical and laboratory abnormalities. Brain 1994;117:1385-1393.

39. Oh SJ. Physiological factors affecting nerve conduction. In: Oh SJ, editor. Clinical electromyography. Nerve conduction studies. Baltimore: Williams \& Wilkins; 1993. p 310

40. Oh SJ. Required tests for specific problems. In: Oh SJ, editor. Clinical electromyography. Nerve conduction studies. BaltiClinical electromyography. Nerve con
more: Williams \& Wilkins; 1993. p 90.

41. Pestronk A, Cornblath DR, Ilyas AA, Baba H, Quarles RH, Griffin JW, Alderson $\mathrm{K}$, Adams RN. A treatable multifocal neuropathy with antibodies to GM1 ganglioside. Ann Neurol 1988;24:73-78.

42. Pestronk A, Li F, Griffin J, Feldman EL, Cornblath DR, Trotter J, Zhu S, Yee WC,Phillips D, Peeples DM, Winslow BS. Polyneuropathy syndromes associated with serum antibodies to sulfatide and myelin-associated glycoprotein. Neurology 1991;41:357-362.

43. Sherman WH, Latov N, Hays AP, Takatsu M, Nemni R, Galassi G, Osserman EF. Monoclonal IgMK antibody precipitating with chondroitin sulphate $C$ from patients with axonal polyneuropathy and epidermolysis. Neurology 1983;33:192-201.

44. Simmons Z, Albers JW, Bromberg MB, Feldman E. Long-term follow-up of pacients with chronic inflammatory demyelinatfollow-up of patients with chronic inflammatory demyelinating polyradiculoneuropathy, without

45. Simovic D, Gorson KC, Ropper AH. Comparison of IgMMGUS and IgG-MGUS polyneuropathy. Acta Neurol Scand 1998;97:194-200.

46. Smith IS. The natural history of chronic demyelinating neu- ropathy associated with benign IgM paraproteinaemia. A clinical and neurophysiological study. Brain 1994;1.17: 949-957.

47. Smith IS, Khan SN, Lacey BW, King RHM, Eames RA, Why brew DJ, Thomas PK. Chronic demyelinating neuropathy associated with benign IgM paraproteinaemia. Brain 1983;106. 169-195.

48. Suarez GA, Kelly J Jr. Polyneuropathy associated with monoSuarez GA, Kelly J Jr. Polyneuropathy associated with mono-
clonal gammopathy of undetermined significance: further clonal gammopathy of undetermined significance: further
evidence that IgM-MGUS neuropathies are different than evidence that IgM-MGUS neuropathies are
IgG-MGUS. Neurology 1993;43:1304-1308

49. Takatsu M, Hays AP, Latov N, Abrams GM, Nemni R, Sherman WH, Nobile-Orazio E, Saito T, Freddo L. Immunofluorescence study of patients with neuropathy and IgM M proteins. Ann Neurol 1985;18:173-181.

50. Tatum AH. Experimental paraprotein neuropathy, demyelination by passive transfer of human IgM anti-myelin-associated glycoprotein. Ann Neurol 1993;33:502-506.

51. Thomas PK, Willison HJ. Paraproteinaemic neuropathy. Baillière's Clinical Neurology 1994;1:129-147.

52. Towbin $H$, Staehelin $T$, Gordon J. Electrophoretic transfer of proteins from polyacrylamide gels to nitrocellulose sheets: procedure and some applications. Proc Nat Acad Sci USA 1979;76:4350-4354

53. Trojaborg W, Hays AP, van den Berg L, Younger DS, Latov N Motor conduction parameters in neuropathies associated with anti-MAG antibodies and other types of demyelinating and axonal neuropathies. Muscle Nerve 1995;18:730-735.

54. Valldeoriola F, Graus F, Steck AJ, Munoz E, de la Fuente $M$ Gallart T, Ribalta T, Bombi JA, Tolosa E. Delayed appearance of anti-myelin-associated glycoprotien antibodies in a patien with chronic demyelinating polyneuropathy. Ann Neurol 1993;34:394-396

55. van den Berg LH, Lankamp CLAM, de Jager AEJ, Notermans NC, Sodaar P, Marrink J, de Jong HJ, Bar PR, Wokke JHJ. Anti-sulphatide antibodies in peripheral neuropathy. J Neurol Anti-sulphatide antibodies in peripheral neurc

56. Vital A, Vital C, Julien J, Baquey A, Steck AJ. Polyneuropathy associated with IgM monoclonal gammopathy. Immunological and pathological study in 31 patients. Acta Neuropatho (Berl) 1989;79:160-167.

57. Willison HJ, Paterson G, Veitch J, Inglis G, Barnett SC. Pe ripheral neuropathy with monoclonal IgM anti-Pr2 cold agglutinins. J Neurol Neurosurg Psychiatry 1993;56:1178-1 183.

58. Willison HJ, Trapp BD, Bacher JD, Dalakas MC, Griffin JW Quarles RH. Demyelination induced by intraneural injection of human anti-myelin-associated glycoprotein antibodies. Muscle Nerve 1988;11:1169-1176.

59. Yee WC, Hahn AF, Hearn SA, Rupar AR. Neuropathy in IgM lambda paraproteinaemia. Immunoreactivity to neural proteins and chondroitin sulfate. Acta Neuropathol (Berl) 1989; 78:57-64.

60. Yeung KB, Thomas PK, King RHM, Waddy H, Will RG Hughes RAC, Gregson NA, Leibowitz S. The clinical spectrum of peripheral neuropathies associated with benign monoclonal IgM, IgC and IgA paraproteinaemia. Comparative clinical, immunological and sural nerve biopsy findings. J Neurol 1991;238:383-391. 